\title{
Exploring supersymmetry and naturalness in light of new experimental data
}

\author{
by
}

\author{
Kevin Earl \\ A thesis submitted to the \\ Faculty of Graduate and Postdoctoral Affairs \\ in partial fulfillment of the requirements \\ for the degree of \\ Doctor of Philosophy in Physics \\ Department of Physics \\ Carleton University \\ Ottawa-Carleton Institute for Physics \\ Ottawa, Canada \\ August 27, 2019 \\ Copyright (c) 2019 Kevin Earl
}




\section{Abstract}

This thesis investigates extensions of the Standard Model (SM) that are based on either supersymmetry or the Twin Higgs model. New experimental data, primarily collected at the Large Hadron Collider (LHC), play an important role in these investigations. Specifically, we examine the following five cases.

We first consider Mini-Split models of supersymmetry. These types of models can be generated by both anomaly and gauge mediation and we examine both cases. LHC searches are used to constrain the relevant parameter spaces, and future prospects at LHC 14 and a $100 \mathrm{TeV}$ proton proton collider are investigated.

Next, we study a scenario where Higgsino neutralinos and charginos are pair produced at the LHC and promptly decay due to the baryonic $R$-parity violating superpotential operator $\lambda^{\prime \prime} U^{c} D^{c} D^{c}$. More precisely, we examine this phenomenology in the case of a single non-zero $\lambda_{3 j k}^{\prime \prime}$ coupling. By recasting an experimental search, we derive novel constraints on this scenario.

We then introduce an $R$-symmetric model of supersymmetry where the $R$ symmetry can be identified with baryon number. This allows the operator $\lambda^{\prime \prime} U^{c} D^{c} D^{c}$ in the superpotential without breaking baryon number. However, the $R$-symmetry will be broken by at least anomaly mediation and this reintroduces baryon number violation. Under these conditions, we investigate baryon number violating processes and flavour physics, as well as the collider phenomenology of both stops and the first two generations of squarks assuming they decay due to a single non-zero $\lambda_{3 j k}^{\prime \prime}$ coupling.

There have been several experimental anomalies related to the quark level transition $b \rightarrow s \ell$. We investigate these anomalies with the $R$-parity violating superpotential operator $\lambda^{\prime} L Q D^{c}$. By examining diagrams featuring winos we find new pa- 
rameter space capable of explaining these anomalies while at the same time avoiding other experimental bounds.

Finally, a variation of the Twin Higgs model is presented. These models stabilize the electroweak scale with particles uncharged under the SM colour group. This is accomplished by introducing a mirror sector to the SM and requiring an approximate $\mathbb{Z}_{2}$ symmetry between the two sectors. It is shown that it is beneficial to break the $\mathbb{Z}_{2}$ symmetry spontaneously rather than explicitly. 


\section{Acknowledgments}

This thesis would not be possible without the considerable support I have received from many different people during my graduate studies at Carleton University. As such, I would like to acknowledge those who have helped me during the last six years. I apologize in advance to anyone who I have missed.

First and foremost, I would like to express my immense gratitude to my supervisor Dr. Thomas Grégoire. I have benefitted greatly from not only his deep understanding of elementary particle physics, but also his unending patience and willingness to assist and guide me in my studies.

Next, I would like to personally thank Dr. Hugues Beauchesne. During the first three years of my graduate studies, Hugues and I worked very closely on research projects. Perhaps the most important thing Hugues taught me during this time was the dedication and work ethic required to truly perform innovative research.

I would also like to thank the other faculty members of the theory group: Dr. Heather Logan, Dr. Bruce Campbell, Dr. Stephen Godfrey, Dr. Daniel Stolarski, and Dr. Pat Kalyniak. Also deserving of recognition are my fellow graduate students: Katy Hartling, Terry Pilkington, Paul Smith, Dylan Linthorne, Jerome Claude, Rouzbeh Modarresi Yazdi, and Alex Poulin. I would also like to acknowledge the administrative staff of the physics department, particularly Joanne Martin and Karina Auclair for helping me print tutorial materials for PHYS 1007 and PHYS 1008.

I would also like to acknowledge the Natural Sciences and Engineering Research Council of Canada (NSERC) and the government of Ontario for providing very generous and much appreciated financial assistance.

Finally, I would like to thank my family: my parents Cheryl and David, my older brothers Brian and Steven, and my younger sister Megan. 


\section{Statement of Originality}

Chapters 2 and 3 present reviews of the Standard Model and supersymmetry, respectively. The information contained in these chapters is well known and can be found in the references provided. Other than reviewing the preexisting literature, chapters 4 , $5,6,7$, and 8 present new results derived from original research. Each of these chapters are based on publications except chapter 5 which is based on currently ongoing work. We list below which chapters correspond to which publications as well as detail the contributions made by myself and my collaborators to each of these projects.

- The results presented in chapter 4 are published in [1]. This project was also worked on by Hugues Beauchesne, a more senior graduate student. As such, the results from this chapter are also presented in Hugues Beauchesne's thesis. All results were individually derived (and cross-checked) by both of us except for the following points.

- The gaugino pole mass calculations for both anomaly and gauge mediation presented in section 4.2.2 were computed by Hugues Beauchesne.

- The writing of codes to recast experimental searches was split between the author and Hugues Beauchesne.

- The scripts used to determine the efficiencies for different topologies to pass the cuts of experimental searches were written by the author.

- The results presented in chapter 5 are based on work that has yet to be published. All results in this chapter were derived by the author.

- The results presented in chapter 6 are published in [2]. The bounds on the $\lambda^{\prime \prime}$ couplings derived from baryon number violating processes and flavour physics 
presented in section 6.2.1 were initially computed by Thomas Grégoire and were independently verified by the author. Except for the bounds coming from displaced vertices which were determined by Hugues Beauchesne, the analysis of the collider phenomenology presented in section 6.3 was performed by the author.

- The results presented in chapter 7 are published in [3]. All results in this chapter were derived by the author.

- The results presented in chapter 8 are published in [4]. This project was also worked on by Hugues Beauchesne. As such, the results from this chapter are also presented in Hugues Beauchesne's thesis. The main idea in this chapter, to break the $\mathbb{Z}_{2}$ symmetry spontaneously, was thought of by Hugues Beauchesne. Many of the details of the model were also initially worked out by Hugues Beauchesne and subsequently confirmed by the author. The radiative corrections presented in section 8.2.3 were originally computed by the author and confirmed by Hugues Beauchesne.

Except for the reservations described above, the research presented in this thesis is new and original (to my knowledge) and has not been published or submitted as a final project by any other individual. 


\section{Contents}

1 Introduction 1

2 The Standard Model $\quad 8$

2.1 Introduction . . . . . . . . . . . . . . . 8

2.2 The symmetries of spacetime . . . . . . . . . . . . . . 9

2.3 Quantum fields and their Lagrangians . . . . . . . . . . . . . . . . . 12

2.4 Non-abelian gauge theories . . . . . . . . . . . . . . . . . . 22

2.5 Field content of the Standard Model . . . . . . . . . . . . . . . . . . 24

2.6 Electroweak symmetry breaking . . . . . . . . . . . . . . . . . . 27

2.7 The Standard Model as an effective field theory and the hierarchy

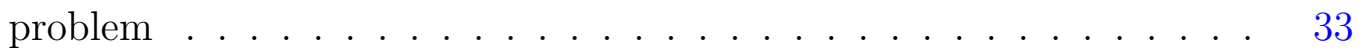

3 Supersymmetry $\quad 35$

3.1 Introduction . . . . . . . . . . . . . . . . . . . 35

3.2 The supersymmetry algebra . . . . . . . . . . . . . . . 37

3.3 Superfields . . . . . . . . . . . . . . . . . . . . . . . . . 40

3.3.1 Chiral superfields . . . . . . . . . . . . . . . 42

3.3.2 Vector superfields ................... 44

3.4 Supersymmetrically invariant Lagrangians . . . . . . . . . . . . . . 47 
3.4.1 Kinetic terms for chiral superfields . . . . . . . . . . . . 48

3.4 .2 Kinetic terms for vector superfields . . . . . . . . . . . 50

3.4 .3 The superpotential . . . . . . . . . . . . . . . . . . 52

3.5 Soft supersymmetry breaking terms . . . . . . . . . . . 53

3.6 The scalar potential . . . . . . . . . . . . . . . . . . . . 54

3.7 The MSSM $\ldots \ldots \ldots \ldots \ldots \ldots$

3.7 .1 Kinetic terms . . . . . . . . . . . . . . . 55

3.7 .2 Superpotential . . . . . . . . . . . . . . . . 58

3.7.3 Soft supersymmetry breaking terms . . . . . . . . . . 59

3.7.4 Electroweak symmetry breaking . . . . . . . . . . . . . 61

3.8 Mediation mechanisms . . . . . . . . . . . . . . . . . . . 64

3.8 .1 Gravity mediation . . . . . . . . . . . . 65

3.8 .2 Gauge mediation . . . . . . . . . . . . . . . . 66

3.8 .3 Anomaly mediation . . . . . . . . . . . . . . 67

$3.9 \quad R$-symmetries . . . . . . . . . . . . . . . . . . . . . 69

4 Phenomenology of Mini-Split supersymmetry 72

4.1 Introduction . . . . . . . . . . . . . . . . . 72

4.2 Theory . . . . . . . . . . . . . . . . . . 74

4.2 .1 Mini-Split models . . . . . . . . . . . . . . . . . . . . 74

4.2 .2 Gaugino mass spectrum $\ldots \ldots \ldots \ldots$

4.2 .3 Gaugino decays . . . . . . . . . . . . . . . 80

4.2 .4 Higgs mass . . . . . . . . . . . . . . . . . 81

4.3 Methodology and results . . . . . . . . . . . . . . 83

4.3 .1 Parameter space . . . . . . . . . . . . . . . . 83

4.3 .2 Current LHC constraints . . . . . . . . . . . . . . . 86 
4.3 .3 Prospects at LHC $14 \ldots \ldots \ldots \ldots$

4.3.4 Prospects at a $100 \mathrm{TeV}$ collider . . . . . . . . . . 97

4.4 Conclusion . . . . . . . . . . . . . . . . . . . . . . . . . . . . . . 101

5 Phenomenology of Higgsinos with baryonic $R$-parity violation at the $\begin{array}{ll}\text { LHC } & 103\end{array}$

5.1 Introduction . . . . . . . . . . . . . . . . . . 103

5.2 Majorana versus Dirac Higgsinos _. . . . . . . . . . . . 106

5.3 Higgsino masses . . . . . . . . . . . . . . . . . . . . . . . 114

5.4 Constraining Higgsinos at the LHC . . . . . . . . . . . 117

5.4 .1 Higgsino decays . . . . . . . . . . . . . . . . . . . . 118

5.4.2 Constraining points in the parameter space . . . . . . . 120

5.4 .3 Parameter scan . . . . . . . . . . . . . . . . . . . . . 122

5.4 .4 Results. . . . . . . . . . . . . . . . . . . 125

5.5 Conclusion . . . . . . . . . . . . . . . . . . . . . . . . . . . . . . 129

6 Phenomenology of supersymmetric models with a $\mathrm{U}(1)_{R}$ baryon number 131

6.1 Introduction . . . . . . . . . . . . . . . . 131

6.2 The model . . . . . . . . . . . . . . . . . . . . . . . . . . . . 133

6.2 .1 Bounds on $\lambda^{\prime \prime} \ldots \ldots \ldots \ldots \ldots \ldots \ldots$

6.2.1.1 Bounds from baryon number violating processes . . . 136

6.2.1.2 Bounds from flavour physics . . . . . . . . . . . 139

6.2.2 Spectrum and parameter space . . . . . . . . . . . 140

6.3 Collider constraints . . . . . . . . . . . . . . . . . . . . 142

6.3 .1 Placing limits on stops . . . . . . . . . . . . . . . 143 
6.3.1.1 Stop production . . . . . . . . . . . . . 143

$6.3 .1 .2 \quad$ Stop LSP . . . . . . . . . . . . . . . . 145

6.3.1.3 Neutralino LSP . . . . . . . . . . . . . . . . 151

6.3.2 Placing limits on first and second generation squarks . . . . 161

6.3.2.1 Squark production . . . . . . . . . . . . . 161

6.3.2.2 Neutralino LSP . . . . . . . . . . . . . . . 165

6.4 Conclusion . . . . . . . . . . . . . . . . . . . . . . . . . . . 168

7 Contributions to $b \rightarrow s \ell \ell$ anomalies from $R$-parity violating interac$\begin{array}{ll}\text { tions } & 171\end{array}$

7.1 Introduction . . . . . . . . . . . . . . . . . . . . 171

7.2 Setup and calculations . . . . . . . . . . . . . . . 174

7.3 Constraints . . . . . . . . . . . . . . . . . . . 185

$7.3 .1 \tau$ decays . . . . . . . . . . . . . . 185

$7.3 .2 \quad$ B mesons . . . . . . . . . . . . . . . . . . . . 190

$7.3 .3 \quad Z$ decays . . . . . . . . . . . . . . . . . . 193

7.3 .4 Other possible decays . . . . . . . . . . . . . 195

7.3.5 Collider searches . . . . . . . . . . . . . . . 196

7.3 .6 Landau poles . . . . . . . . . . . . . . . . . . . 198

7.4 Results . . . . . . . . . . . . . . . . . . . . . . . . 198

7.4 .1 Additional remarks . . . . . . . . . . . . . . . . . . 201

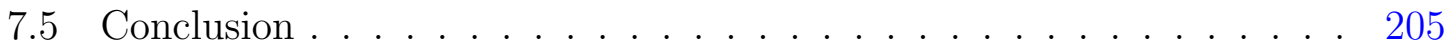

8 The spontaneous $\mathbb{Z}_{2}$ breaking Twin Higgs 208

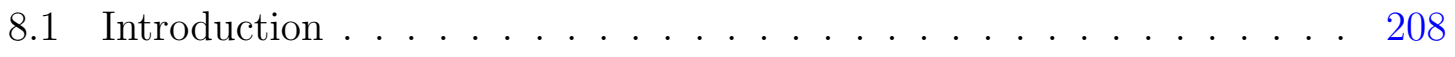

8.2 The model . . . . . . . . . . . . . . . . . . . . 210 
8.2.1 The original Twin Higgs _ . . . . . . . . . . . . . . 210

$8.2 .2 \quad$ Spontaneous $\mathbb{Z}_{2}$ breaking . . . . . . . . . . . . . . . 214

8.2.2.1 Potential and vevs . . . . . . . . . . . . 215

8.2.2.2 Small $\alpha_{i}$ 's approximation . . . . . . . . 216

8.2.2.3 Additional properties . . . . . . . . . . . . . . 221

8.2.3 Radiative corrections . . . . . . . . . . . . . . . . . . . 223

8.3 Numerical analysis of the fine-tuning . . . . . . . . . . . 225

8.4 Conclusion . . . . . . . . . . . . . . . . . . . . . . . . . . . . 229

9 Summary 232

A One loop beta functions used in the investigation of $b \rightarrow s \ell \ell$ anoma$\begin{array}{ll}\text { lies } & 236\end{array}$

$\begin{array}{ll}\text { References } & 240\end{array}$ 


\section{List of Tables}

4.1 Gluino pair production searches. . . . . . . . . . . . . 86

4.2 Signal regions for high MET. The background for $3 \mathrm{ab}^{-1}$ is also included. 98

5.1 Experimental search recasted for this analysis. . . . . . . . . . . . 121

$6.1 R$-charge assignment of chiral superfields of the model. . . . . . . . . 134

6.2 Neutralino LSP searches. For searches [215,217], we use FastJet [77, 220] for manipulating large $(R=1)$ jets. This mainly involves jet reclustering and jet trimming. Additionally, searches that feature missing transverse energy (MET) either have very lenient cuts on this quantity or also contain signal regions probing $R$-parity conserving supersymmetry signatures. . . . . . . . . . . . . . . . 158 


\section{List of Figures}

3.1 Quadratically divergent one loop diagrams with top quarks and squarks contributing to the squared mass of the Higgs. The quadratic divergences cancel between the two diagrams. . . . . . . . . . . .

3.2 Example diagrams generating MSSM gaugino (3.2a) and scalar (3.2b and 3.2c) soft supersymmetry breaking mass terms from gauge mediation. In these diagrams, $\lambda$ and $\phi$ represent MSSM gauginos and scalars, respectively, and wavy lines represent SM gauge bosons. Additionally, the thick solid and dashed lines represent the heavy fermionic and scalar messengers, respectively. . . . . . . . . . . . .

4.1 Typical mass spectrum for (a) anomaly mediation and (b) gauge mediation. In (a), the masses appearing on the right side of equation 4.10 are taken to be $m_{\text {scalars }}=\mu=m_{3 / 2}=50 \mathrm{TeV}$ with $\tan \beta=2$. In (b), the masses appearing on the right side of equation 4.13 are taken to be $m_{\text {scalars }}=\mu=\Lambda=200 \mathrm{TeV}$ with $\tan \beta=2 \ldots \ldots$. . . . .

4.2 Branching ratios of the gluino for $M_{\tilde{G}}=1500 \mathrm{GeV}$ and $M_{\tilde{B}}=0 \mathrm{GeV}$. The third generation scalar masses are assumed to be degenerate and much heavier than the gauginos. . . . . . . . . . . . . . 
4.3 95\% exclusion limits for anomaly mediation at the LHC. The yellow band corresponds to the $1 \sigma$ uncertainty on the gluino pair production cross section and the purple bands are the forbidden region of colourbreaking vacuum. Contour lines of constant $M_{\tilde{B}}, M_{\tilde{W}}, \mu$, and $\tan \beta$ are shown respectively in $(\mathrm{a}),(\mathrm{b}),(\mathrm{c})$, and $(\mathrm{d}) \ldots \ldots \ldots$

$4.495 \%$ exclusion limits for gauge mediation at the LHC. The yellow band corresponds to the $1 \sigma$ uncertainty on the gluino pair production cross section and the purple bands are the forbidden region of colour-breaking vacuum. The grey band corresponds to limits from direct electroweak searches. Contour lines of constant $M_{\tilde{B}}, M_{\tilde{W}}, \mu$, and $\tan \beta$ are shown respectively in (a), (b), (c), and (d). . . . . . .

4.5 95\% exclusion limits for anomaly mediation at LHC 14 for (solid) 300 $\mathrm{fb}^{-1}$ and (dashed) $3000 \mathrm{fb}^{-1}$ integrated luminosity. The green band corresponds to the $1 \sigma$ uncertainty on the gluino pair production cross section for $300 \mathrm{fb}^{-1}$, the yellow band corresponds to the $1 \sigma$ uncertainty on the gluino pair production cross section for $3000 \mathrm{fb}^{-1}$, and the purple bands are the forbidden region of colour-breaking vacuum. Contour lines of constant $M_{\tilde{B}}, M_{\tilde{W}}, \mu$, and $\tan \beta$ are shown respectively in (a), $(\mathrm{b}),(\mathrm{c})$, and $(\mathrm{d}) \ldots \ldots \ldots \ldots \ldots$ 
4.6 $5 \sigma$ discovery limits for anomaly mediation at LHC 14 for (solid) 300 $\mathrm{fb}^{-1}$ and (dashed) $3000 \mathrm{fb}^{-1}$ integrated luminosity. The green band corresponds to the $1 \sigma$ uncertainty on the gluino pair production cross section for $300 \mathrm{fb}^{-1}$, the yellow band corresponds to the $1 \sigma$ uncertainty on the gluino pair production cross section for $3000 \mathrm{fb}^{-1}$, and the purple bands are the forbidden region of colour-breaking vacuum. Contour lines of constant $M_{\tilde{B}}, M_{\tilde{W}}, \mu$, and $\tan \beta$ are shown respectively in (a), (b), (c), and $(\mathrm{d}) \ldots \ldots \ldots \ldots \ldots \ldots$

4.7 95\% exclusion limits for gauge mediation at LHC 14 for (solid) 300 $\mathrm{fb}^{-1}$ and (dashed) $3000 \mathrm{fb}^{-1}$ integrated luminosity. The green band corresponds to the $1 \sigma$ uncertainty on the gluino pair production cross section for $300 \mathrm{fb}^{-1}$, the yellow band corresponds to the $1 \sigma$ uncertainty on the gluino pair production cross section for $3000 \mathrm{fb}^{-1}$, and the purple bands are the forbidden region of colour-breaking vacuum. Contour lines of constant $M_{\tilde{B}}, M_{\tilde{W}}, \mu$, and $\tan \beta$ are shown respectively in (a), $(\mathrm{b}),(\mathrm{c})$, and $(\mathrm{d}) . \ldots \ldots \ldots \ldots$

$4.85 \sigma$ discovery limits for gauge mediation at LHC 14 for (solid) 300 $\mathrm{fb}^{-1}$ and (dashed) $3000 \mathrm{fb}^{-1}$ integrated luminosity. The green band corresponds to the $1 \sigma$ uncertainty on the gluino pair production cross section for $300 \mathrm{fb}^{-1}$, the yellow band corresponds to the $1 \sigma$ uncertainty on the gluino pair production cross section for $3000 \mathrm{fb}^{-1}$, and the purple bands are the forbidden region of colour-breaking vacuum. Contour lines of constant $M_{\tilde{B}}, M_{\tilde{W}}, \mu$, and $\tan \beta$ are shown respectively in (a), $(\mathrm{b}),(\mathrm{c})$, and $(\mathrm{d}) \ldots \ldots \ldots \ldots \ldots$ 
$4.995 \%$ (dashed) exclusion and $5 \sigma$ (solid) discovery limits for anomaly mediation at a $100 \mathrm{TeV} p p$ collider with $3 \mathrm{ab}^{-1}$ integrated luminosity. The yellow band corresponds to the $1 \sigma$ uncertainty on the gluino pair production cross section for $95 \%$ exclusion, the green band corresponds to the $1 \sigma$ uncertainty on the gluino pair production cross section for $5 \sigma$ discovery, and the purple bands are the forbidden region of colourbreaking vacuum. Contour lines of constant $M_{\tilde{B}}, M_{\tilde{W}}, \mu$, and $\tan \beta$ are shown respectively in (a), (b), (c), and (d) . . . . . . . . . .

$4.1095 \%$ (dashed) exclusion and $5 \sigma$ (solid) discovery limits for gauge mediation at a $100 \mathrm{TeV} p p$ collider with $3 \mathrm{ab}^{-1}$ integrated luminosity. The yellow band corresponds to the $1 \sigma$ uncertainty on the gluino pair production cross section for $95 \%$ exclusion, the green band corresponds to the $1 \sigma$ uncertainty on the gluino pair production cross section for $5 \sigma$ discovery, and the purple bands are the forbidden region of colourbreaking vacuum. Contour lines of constant $M_{\tilde{B}}, M_{\tilde{W}}, \mu$, and $\tan \beta$ are shown respectively in (a), (b), (c), and (d). . . . . . . . 100

5.1 Possible decays of a Dirac Higgsino due to $\lambda_{3 j k}^{\prime \prime}$. The top row shows the diagrams for $\chi_{D}^{0} \rightarrow t d_{j} d_{k}$ and the bottom row shows the diagrams for $\chi_{D}^{0} \rightarrow \bar{t} \bar{d}_{j} \bar{d}_{k} \ldots \ldots \ldots \ldots \ldots \ldots \ldots \ldots \ldots \ldots \ldots \ldots \ldots \ldots$ 
5.2 Example figures showing the widths and branching ratios for neutral Higgsinos as functions of the stop mixing angle. Figures 5.2a shows the decay widths for the Higgsino treated as a Dirac state. Figure 5.2b is similar to $5.2 \mathrm{a}$, except the Higgsino is now treated as two Majorana states. Figures 5.2c and 5.2d shows the branching ratios into opposite sign and same sign top final states assuming Higgsino pair production at the LHC. Figure 5.2c treats the pair produced Higgsinos as Dirac particles and is produced by using the partial widths in figure $5.2 \mathrm{a}$ together with the narrow width approximation. Figure 5.2d treats the pair produced Higgsinos as Majorana particles and is produced with the assistance of MadGraph. For each of these figures, we have set $\lambda_{312}^{\prime \prime}=1, \tan \beta=40, \mu=500 \mathrm{GeV}, m_{\tilde{t}_{1}}=800 \mathrm{GeV}$, and $m_{\tilde{t}_{2}}=$ $1400 \mathrm{GeV} \ldots \ldots \ldots \ldots \ldots \ldots$

5.3 Branching ratios into final states with opposite sign and same sign tops for a $\chi_{S}^{0} \chi_{A}^{0}$ pair produced at the LHC as a function of the mass splitting between the states. Figure 5.3a considers zero stop mixing and figure $5.3 \mathrm{~b}$ considers maximally mixed stops. The other parameters are set to the same values as in figure 5.2. Both figures have been produced with MadGraph. . . . . . . . . . . . . . . . .

5.4 Excluded points found by the parameter scan projected onto different axes. Figure 5.4a shows the Higgsino masses, where $m_{\chi_{1(2)}^{0}}$ is the mass of the lighter (heavier) neutralino, and figures $5.4 \mathrm{~b}, 5.4 \mathrm{c}, 5.4 \mathrm{~d}$, and $5.4 \mathrm{e}$ show, respectively, $\lambda_{3 j 3}^{\prime \prime}$ and $\mu, M_{1}$ and $M_{2}$, the stop masses and the stop mixing angle, and the sbottom masses and the sbottom mixing angle. . . . . . . . . . . . . . . . . 
5.5 Exclusion curves in the $\mu$ and $\lambda_{3 j 3}^{\prime \prime}$ plane. Figure 5.5 a sets $m_{D_{3}}=1 \mathrm{TeV}$ and shows curves for three different vales of $m_{U_{3}}$. Figure $5.5 \mathrm{~b}$ sets $m_{U_{3}}=3 \mathrm{TeV}$ and shows curves for three different vales of $m_{D_{3}}$. The other parameters have been set to $M_{1}=M_{2}=1 \mathrm{TeV}, \tan \beta=10$, $m_{Q_{3}}=2 \mathrm{TeV}$, and $A_{t}=A_{b}=0$. The region within each curve is excluded. . . . . . . . . . . . . . . . . .

6.1 Diagrams leading to neutron antineutron oscillation. Flavour changing insertions are needed on the squark lines and a Majorana mass insertion is needed on the gluino line. (a) shows a diagram with flavour changing insertions of the right-handed squarks. (b) shows a diagram requiring a left-right squark mixing which is further suppressed by the gravitino mass. . . . . . . . . . . . . . . . . . .

6.2 Diagram mediating the $p p \rightarrow K^{+} K^{+}$process. . . . . . . . . . . 138

6.3 Decay of the stop through the two on-shell pseudo-Dirac states $\chi_{1}^{B}$ and $\chi_{2}^{B}$. When the mass difference between the two states is smaller than the width, the diagram with $\chi_{1}^{B}$ cancels the one with $\chi_{2}^{B}$. . . . . 142

6.4 Stop production at centre of mass energy of 8 and 13 TeV. Here $\sigma(\mathrm{pp} \rightarrow$ stop) stands for $\sigma\left(\mathrm{pp} \rightarrow \tilde{t}^{*}\right)+\sigma(\mathrm{pp} \rightarrow \tilde{t})$. For resonant stop production, only one $\lambda_{3 j k}^{\prime \prime}$ is non-zero at a time. . . . . . . . . . . . . . . . 145

6.5 Acceptances for the dijet searches recasted in this analysis. Top row: ATLAS searches. Bottom row: CMS searches. . . . . . . . . . . . . . 148

6.6 Exclusion plot for an LSP stop. The area above each curve is excluded by dijet searches. Additionally, the grey area is excluded by stop pair production with subsequent decay into four quarks. As explained in the text, it applies equally to all $\lambda_{3 j k}^{\prime \prime} \ldots \ldots$. . . . . . . . . 150 
6.7 Branching ratios for a $600 \mathrm{GeV}$ stop as a function of of $\lambda_{312}^{\prime \prime}$. For both plots, the neutralino mass has been set to $200 \mathrm{GeV}$. . . . . . . . . . 152

6.8 Partial decay widths (a) and branching ratios (b) for $600 \mathrm{GeV}$ stops decaying into opposite sign (OS) and same sign (SS) tops through approximately $200 \mathrm{GeV}$ bino neutralinos with $\lambda_{312}^{\prime \prime}=1$. . . . . . . . 154

6.9 The efficiency for reconstructing a single vertex $f(m, c \tau)$ for (a) fixed $m=1 \mathrm{TeV}$ and (b) fixed $c \tau=0.1 \mathrm{~m}$ for the CMS search [222]. . . . 160

6.10 Exclusion plots for a $200 \mathrm{GeV}$ bino neutralino LSP with the $\mathrm{U}(1)_{R}$ symmetry strictly preserved. The grey region on the left side of the plots $\left(m_{\tilde{t}} \lesssim 375 \mathrm{GeV}\right)$ is excluded by paired dijet searches. Next, consider the middle region of the plots. Starting from large $\lambda_{3 j k}^{\prime \prime}$ couplings and working downwards, the blue region is excluded by dijet searches, the green region is excluded by neutralino LSP searches, and the red regions are excluded by displaced vertices searches. Bino pair production, which contributes to the displaced vertices limits, depends on the masses of the first and second generations of squarks. Setting the masses of these squarks to $10 \mathrm{TeV}$ results in the darker red region being excluded. Instead, setting the masses of these squarks to $1 \mathrm{TeV}$ excludes both the darker red and lighter red regions. . . . . . . . . . 162 
6.11 Exclusion plots for a $200 \mathrm{GeV}$ Higgsino-up neutralino LSP with the $\mathrm{U}(1)_{R}$ symmetry strictly preserved. The grey region on the left side of the plots $\left(m_{\tilde{t}} \lesssim 205 \mathrm{GeV}\right)$ is excluded by paired dijet searches. Next, consider the middle region of the plots. Starting from large $\lambda_{3 j k}^{\prime \prime}$ couplings and working downwards, the blue region is excluded by dijet searches, the green region is excluded by neutralino LSP searches, and the red region is excluded by displaced vertices searches. . . . . . . . 163

6.12 Exclusion plots for a $200 \mathrm{GeV}$ bino neutralino LSP with the $\mathrm{U}(1)_{R}$ symmetry broken. The grey region on the left side of the plots $\left(m_{\tilde{t}} \lesssim 375\right.$ $\mathrm{GeV}$ ) is excluded by paired dijet searches. Next, consider the middle region of the plots. Starting from large $\lambda_{3 j k}^{\prime \prime}$ couplings and working downwards, the blue region is excluded by dijet searches, the green region is excluded by neutralino LSP searches, and the red regions are excluded by displaced vertices searches. Bino pair production, which contributes to the displaced vertices limits, depends on the masses of the first and second generations of squarks. Setting the masses of these squarks to $10 \mathrm{TeV}$ results in the darker red region being excluded. Instead, setting the masses of these squarks to $1 \mathrm{TeV}$ excludes both the darker red and lighter red regions. . . . . . . . . . . . . . . . . 164 
6.13 Exclusion plots for a $200 \mathrm{GeV}$ Higgsino-up neutralino LSP with the $\mathrm{U}(1)_{R}$ symmetry broken. The grey region on the left side of the plots $\left(m_{\tilde{t}} \lesssim 205 \mathrm{GeV}\right)$ is excluded by paired dijet searches. Next, consider the middle region of the plots. Starting from large $\lambda_{3 j k}^{\prime \prime}$ couplings and working downwards, the blue region is excluded by dijet searches, the green region is excluded by neutralino LSP searches, and the red region is excluded by displaced vertices searches. . . . . . . . . . . . . . 165

6.14 Exclusion curves within the neutralino-squark mass parameter space for a bino neutralino. Figure (a) presents $\lambda_{312}^{\prime \prime}=1$ while (b) presents either $\lambda_{313}^{\prime \prime}=1$ or $\lambda_{323}^{\prime \prime}=1$. The dashed green curves assume leading order squark production whereas the solid blue curves include MSSM K-factors. . . . . . . . . . . . . . . . 168

6.15 Exclusion curves within the neutralino-squark mass parameter space for a Higgsino-up neutralino. Figure (a) presents $\lambda_{312}^{\prime \prime}=1$ while (b) presents either $\lambda_{313}^{\prime \prime}=1$ or $\lambda_{323}^{\prime \prime}=1$. The dashed green curves assume leading order squark production whereas the solid blue curves include MSSM K-factors. . . . . . . . . . . . . . . . . . . 169

7.1 Tree level decay for $b \rightarrow s \mu \mu$ involving two $\lambda^{\prime}$ interactions. . . . . . . 176

7.2 Box diagrams studied in this chapter. Figure 7.2a shows an example $W$ loop diagram, figure $7.2 \mathrm{~b}$ shows an example wino loop diagram, and figures $7.2 \mathrm{c}$ and $7.2 \mathrm{~d}$ show the four- $\lambda^{\prime}$ loop diagrams. . . . . . . . . . 178

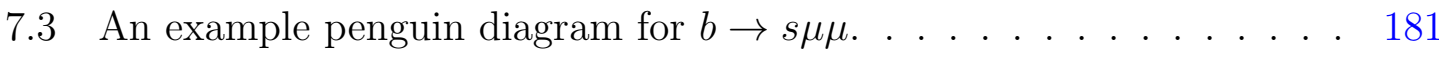

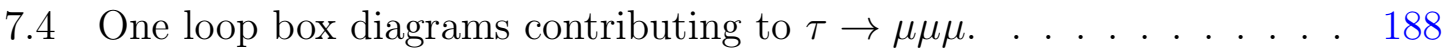

7.5 Example one loop Feynman diagrams contributing to $Z \rightarrow \mu \mu$. . . . 194 
7.6 Four example figures showing solid contours of $C_{L L}^{\mu}$. For figure 7.6a, we set $\lambda_{323}^{\prime}=-\lambda_{333}^{\prime}=1.4, m_{\tilde{W}}=300 \mathrm{GeV}, m_{\tilde{u}_{L}}=m_{\tilde{c}_{L}}=m_{\tilde{t}_{L}}=1.3 \mathrm{TeV}$, and $m_{\tilde{b}_{R}}=m_{\tilde{\nu}_{\mu}}=m_{\tilde{\nu}_{\tau}}=13 \mathrm{TeV}$. For figure $7.6 \mathrm{~b}$, the masses are set to the same values as in figure 7.6a. For figures 7.6c and 7.6d, $\lambda_{323}^{\prime}, \lambda_{333}^{\prime}$, and the masses not being varied are again set to the values used in figure 7.6a. Dashed contours show energy scales of Landau poles in $\mathrm{TeV}$. Parameter space excluded by $\tau \rightarrow \mu \mu \mu$ is shown in yellow. Parameter space excluded by $B_{s}-\bar{B}_{s}$ mixing is shown in blue. Parameter space excluded by $B \rightarrow K^{(*)} \nu \bar{\nu}$ is shown in orange. Finally, parameter space excluded by direct LHC searches is shown in green.

7.7 Example figure showing solid contours of $C_{L L}^{\mu}$ for parameter space similar to that considered in Ref. [276]. For this figure, we set $\lambda_{323}^{\prime}=0.05$, $\lambda_{333}^{\prime}=-0.5, m_{\tilde{W}}=300 \mathrm{GeV}$, and $m_{\tilde{u}_{L}}=m_{\tilde{c}_{L}}=m_{\tilde{t}_{L}}=m_{\tilde{b}_{R}}=m_{\tilde{\nu}_{\mu}}=$ $m_{\tilde{\nu}_{\tau}}=2 \mathrm{TeV}$. Dashed contours show energy scales of Landau poles in $\mathrm{TeV}$. Parameter space excluded by $B_{s}-\bar{B}_{s}$ mixing is shown in blue. Parameter space excluded by $B \rightarrow K^{(*)} \nu \bar{\nu}$ is shown in orange. Finally, parameter space excluded by $Z$ decays to charged leptons is shown in cyan.

8.1 Example of the different vevs as a function of $B_{\mu} / B_{\mu}^{\max }$. The parameters are $\mu_{1}=750 \mathrm{GeV}, \mu_{2}=850 \mathrm{GeV}, \alpha_{1}=-0.15, \alpha_{2}=0.2$, and

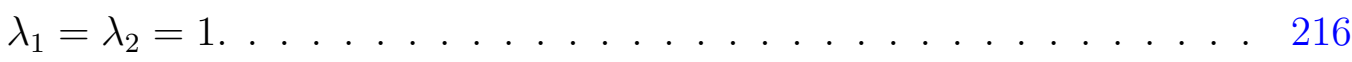

$8.2 F_{1}(\theta)$ and $F_{2}(\theta, \Omega)$ for different values of $\Omega \ldots \ldots \ldots \ldots \ldots \ldots$

$8.3 C\left(-\alpha_{2} / \alpha_{1}, \Omega\right)$ for various values for $-\alpha_{2} / \alpha_{1}$. Also shown is the corresponding value for Twin Higgs. . . . . . . . . . . . . . . . . 220 
8.4 Example of $\Theta_{B}$ for the Twin Higgs and spontaneous $\mathbb{Z}_{2}$ breaking model with different values of $\mu_{2}$. The parameters for the Twin Higgs model are $\mu=750 \mathrm{GeV}, \alpha=-0.15$, and $\lambda=1$. The parameters for the spontaneous $\mathbb{Z}_{2}$ breaking model are $\mu_{1}=750 \mathrm{GeV}, \alpha_{1}=-0.15, \alpha_{2}=$ 0.2, and $\lambda_{1}=\lambda_{2}=1 \ldots \ldots \ldots \ldots \ldots \ldots \ldots$

8.5 Tuning of the spontaneous $\mathbb{Z}_{2}$ breaking Twin Higgs for a fixed $f_{1} / v$ of 3. The left panel shows the tuning in percentage and the right one the ratio of the tuning to the Twin Higgs benchmark of $27.7 \%$. The grey area corresponds to the region where the constraints do not accept any solution. . . . . . . . . . . . . . . . . . 228

8.6 $f_{1} / v$ for the spontaneous $\mathbb{Z}_{2}$ breaking Twin Higgs for a fixed tuning of 20\%. The left panel shows $f_{1} / v$ and the right one the ratio of $f_{1} / v$ to the Twin Higgs benchmark of 3.42. The grey area corresponds to the region where the constraints do not accept any solution. . . . . . . . 228 


\section{Chapter 1}

\section{Introduction}

Elementary particle physics is currently at a crossroads. On the one hand, all elementary particles and their interactions are described incredibly well by the Standard Model (SM). On the other, the SM has a number of deficiencies and it is generally accepted that new physics is required to address them. Besides these problems, which will be discussed in more detail momentarily, there is another more subjective reason to believe that the SM is not all that there is. The SM works up to the highest energy scales currently probed by experiments, approximately $10^{3} \mathrm{GeV}$. However, gravitational interactions, not considered a part of the SM, are described by a non-renormalizable theory up to a cutoff energy scale equal to the Planck scale, $m_{P l} \sim 10^{19} \mathrm{GeV}$. It would be extremely bizarre if no additional physics emerges in the 16 orders of magnitude between the energy scales currently probed and the Planck scale. As has been demonstrated numerous times before, Nature has a tendency to surprise. It is best to keep an open mind when considering what awaits at unexplored energy scales.

The problems of the SM are both practical and theoretical in nature. An example of a practical problem is the need to accommodate the tiny but non-zero masses 
of the neutrinos. Another is to explain dark matter, a form of non-baryonic matter that is known to permeate the Universe and has an energy density that makes up approximately $25 \%$ of the total energy density (and is about 5 times larger than the energy density of ordinary baryonic matter). Another cosmological problem which needs to be addressed is the asymmetry between matter and antimatter in the Universe, so-called baryogenesis. Moving towards the more theoretical side, the strong CP problem, i.e. why the QCD vacuum angle is so small, remains an open question. Another question that can be asked is whether our current vacuum state is absolutely stable or will it eventually decay into a deeper minimum? This follows from the fact that if the masses of the top quark and Higgs boson do not shift too much from their currently measured values, then the SM Higgs quartic coupling runs negative if it is evolved to high energy scales using the SM renormalization group equations. One more open question is why is the cosmological constant so small in natural units?

The problems mentioned in the previous paragraph will not be considered in this thesis. Instead, this thesis will be guided by yet another SM deficiency, known as the hierarchy problem. This is the quadratic sensitivity of the electroweak scale, or, equivalently, the mass of the Higgs boson, to very high energy scales due to radiative corrections in the form of loop diagrams. The standard mechanism to render the electroweak scale natural is for new physics to come in at an energy scale not too far above the electroweak scale and remove the quadratic sensitivity. We say that the new physics stabilizes the hierarchy. Admittedly, there are other potential solutions to the hierarchy problem such as some type of cosmological or anthropic selection principle. Even more so, it is technically possible, however unlikely, that the electroweak scale is simply highly fine-tuned, and that new physics only emerges at energy scales several orders of magnitude larger than the electroweak scale. But until experiments settle 
this issue, it is beneficial to study models capable of solving the hierarchy problem by introducing particles with masses near the electroweak scale, especially since such models do exist.

Perhaps the most famous type of theory that cures the hierarchy problem is supersymmetry (SUSY). Supersymmetry, in general, is a spacetime symmetry that links particles whose spins differ by half a unit. Thus, it links bosons to fermions with these particles being referred to as superpartners of one another. To alleviate the hierarchy problem, the superpartners of the SM particles must have masses not too far above the electroweak scale. Particularly, this setup would stabilize the hierarchy between the electroweak scale and the Planck scale. The superpartners also have the same gauge charges as their SM counterparts and this implies potentially rich phenomenology at modern particle colliders. Indeed, the ATLAS and CMS collaborations at the LHC have set up extensive search programs looking for supersymmetry, and, more generally, any potential signs of new physics, at energies just above the electroweak scale. This has generated a significant amount of new experimental data. One of the main ideas of this thesis will be to determine what this new data says about supersymmetry.

Setting aside naturalness concerns for the moment, supersymmetry also has many other benefits, such as providing dark matter candidates and gauge coupling unification. These latter two benefits are realized by an unnatural model of supersymmetry called Split-SUSY, which is the subject of the first project presented in this thesis. In these types of theories, the scalar superpartners of the SM fermions have very large masses whereas the fermion superpartners of the gauge bosons, the gauginos, have masses not too far above the electroweak scale. However, if the scalar masses become too large, it becomes impossible to accommodate the mass of the 
recently discovered Higgs boson within the model. The amount of splitting is then limited to a few orders of magnitude and the resulting models are called Mini-Split. These types of models can occur due to both anomaly mediation and gauge mediation and we consider both cases. By studying the phenomenology of the gauginos and by recasting current collider searches, constraints are placed on these types of models. Future prospects at LHC 14 and a $100 \mathrm{TeV}$ proton proton collider are also presented.

Returning to natural models of supersymmetry, a general requirement for these types of models is that the masses of the fermionic superpartners of the Higgs doublets, the Higgsinos, should be near the electroweak scale. However, ATLAS and CMS searches for these particles, assuming production either indirectly through the decays of other supersymmetric particles or directly through electroweak processes, continue to put ever increasing lower limits on their masses. The second project presented in this thesis considers a previously unexplored possibility that Higgsino neutralinos and charginos are pair produced at the LHC and promptly decay due to the baryonic $R$ parity violating superpotential operator $\lambda^{\prime \prime} U^{c} D^{c} D^{c}$. More precisely, we assume that there is a single non-zero coupling of the form $\lambda_{3 j k}^{\prime \prime}$ in the mass basis. Pair production of Higgsinos can then lead to same sign tops and this in turn can lead to same sign leptons. By recasting a recent CMS search looking for same sign leptons and multiple b-jets, novel constraints on this scenario are derived. Additionally, subtleties regarding the production of same sign versus opposite sign tops are discussed in detail.

Natural supersymmetric theories can also possess an $R$-symmetry, and this imposes additional structure on the theory. For example, the gauginos are now required to be Dirac instead of Majorana fermions. Moreover, different $R$-charge assignments are possible and lead to different models. The third project presented in this thesis investigates the phenomenology of an $R$-symmetric model of supersymmetry where the 
$R$-symmetry is identified with baryon number. By this we mean that the $R$-charges of the SM particles are simply equal to their standard baryon numbers. However, since $R$-symmetries do not commute with supersymmetry, the superpartners of the SM particles have non-standard baryon numbers. Furthermore, this $R$-symmetry allows the operator $\lambda^{\prime \prime} U^{c} D^{c} D^{c}$ in the superpotential without breaking baryon number. However, the $R$-symmetry cannot remain an exact symmetry of the model as it will be broken by at least anomaly mediation and this reintroduces baryon number violating effects. Under these conditions, the constraints coming from baryon number violating processes and flavour physics are investigated and we find that, in general, they are lessened. As in the Higgsino case, the presence of the $\lambda^{\prime \prime} U^{c} D^{c} D^{c}$ operator in the superpotential considerably changes the collider phenomenology. This project performs a detailed investigation of both stops and the first two generations of squarks assuming they decay due to a single non-zero coupling of the form $\lambda_{3 j k}^{\prime \prime}$ to final states featuring only SM particles. Both limits in which the $R$-symmetry is unbroken and broken are considered as they have different phenomenology and lead to different constraints. This is again due to subtleties regarding the production of opposite sign versus same sign tops, very similar to those encountered in the Higgsino case. Here, these subtleties are now related to conservation of baryon number.

Supersymmetry could also be playing a role in several experimentally observed anomalies in semileptonic $B$ meson decays. Many of these anomalies correspond to the quark level transitions $b \rightarrow s \ell \ell$. These anomalies are the focus of the fourth project in this thesis which attempts to explain them within a supersymmetric framework with the $R$-parity violating superpotential operator $\lambda^{\prime} L Q D^{c}$. To do so, contributions of loop level diagrams to low energy effective operators are calculated. In particular, we concentrate on parameters for which diagrams involving winos give large contri- 
butions. Many different potentially constraining processes are analyzed, including $\tau \rightarrow \mu \mu \mu, B_{s}-\bar{B}_{s}$ mixing, $B \rightarrow K^{(*)} \nu \bar{\nu}, Z$ decays to charged leptons, and direct LHC searches. We find that it is possible to explain the anomalies, but it requires large values of $\lambda^{\prime}$, and this leads to relatively low Landau poles.

The final project presented in this thesis sets aside supersymmetry and instead considers a Twin Higgs type model. These types of models are also capable of stabilizing the electroweak scale but do so in a neutral natural manner. By this we mean that they stabilize the electroweak scale with partner particles that are not charged under the SM gauge groups (or, at the very least, not charged under SM colour). It should be mentioned that these types of models do not stabilize the hierarchy between the electroweak scale and the Planck scale. Instead, they only stabilize the electroweak scale at the one loop level, or, equivalently, up to an energy scale at which two loop radiative corrections become large. At this scale additional physics should come in, such as supersymmetry, to stabilize the hierarchy up to the Planck scale. Thus, Twin Higgs models solve the little hierarchy problem.

The Twin Higgs mechanism works by first extending the Higgs sector so that the scalar potential has an approximate global SU(4) symmetry. This symmetry is then spontaneously broken to $\mathrm{SU}(3)$ resulting in pseudo-Goldstone bosons, one of which is identified with the recently discovered Higgs boson. The extended Higgs fields are part of a mirror sector and a $\mathbb{Z}_{2}$ symmetry between the mirror sector and the SM sector is imposed. The $\mathbb{Z}_{2}$ symmetry is responsible for protecting the mass of the Higgs boson from quadratic corrections at the one loop level. However, the $\mathbb{Z}_{2}$ symmetry must be broken to achieve a phenomenologically viable model and the simplest way to do so is by introducing a soft $\mathbb{Z}_{2}$ breaking mass term. This term needs to be tuned against the $\mathrm{SU}(4)$ breaking terms to produce an acceptable hierarchy of 
vacuum expectation values between the two sectors. This thesis presents a variation of the Twin Higgs model in which the $\mathbb{Z}_{2}$ symmetry is broken spontaneously instead of explicitly. It is demonstrated that this results in many favourable properties, such as less required tuning and a Higgs boson which is very SM like.

This thesis is organized as follows. Reviews of the Standard Model and supersymmetry are presented in chapters 2 and 3, respectively. The phenomenology of Mini-Split models is then studied in chapter 4. Chapter 5 determines the current collider bounds on Higgsino neutralinos and charginos decaying due to the $\lambda^{\prime \prime} U^{c} D^{c} D^{c}$ superpotential operator. The $R$-symmetric model of supersymmetry with the $R$ symmetry identified with baryon number is then studied in chapter 6 . The experimental $B$ anomalies corresponding to the quark level transitions $b \rightarrow s \ell \ell$ are then investigated in chapter 7. Our variation on the Twin Higgs model is presented in chapter 8. Finally, the main results of this thesis are summarized in chapter 9. 


\section{Chapter 2}

\section{The Standard Model}

\section{$2.1 \quad$ Introduction}

The Standard Model (SM) is a quantum field theory based on the non-abelian gauge group $\mathrm{SU}(3)_{\mathrm{C}} \times \mathrm{SU}(2)_{\mathrm{L}} \times \mathrm{U}(1)_{\mathrm{Y}}$. The $\mathrm{C}$ stands for colour, the L stands for left, and the Y stands for hypercharge. It also has chiral fermions and a scalar particle, each of which are charged under some representation of the aforementioned gauge group. The scalar particle acquires a vacuum expectation value and this spontaneously breaks the gauge symmetry down to the subgroup $\mathrm{SU}(3)_{\mathrm{C}} \times \mathrm{U}(1)_{\mathrm{EM}}$.

After the breaking of the electroweak symmetry, three linear combinations of the $\mathrm{SU}(2)_{\mathrm{L}} \times \mathrm{U}(1)_{\mathrm{Y}}$ gauge bosons acquire mass while the fourth linear combination remains massless. The three gauge bosons which acquire mass are the charged $W^{+}$and $W^{-}$and the neutral $Z$, while the massless gauge boson is the photon, the mediator of the electromagnetic force. The gauge bosons for $\mathrm{SU}(3)_{\mathrm{C}}$, called gluons, are unaffected by the electroweak symmetry breaking and stay massless. The chiral fermions also acquire mass after electroweak symmetry breaking and are naturally categorized by their charges under $\mathrm{SU}(3)_{\mathrm{C}}$. The fermions charged under the $\mathrm{SU}(3)_{\mathrm{C}}$ are called 
quarks. There are six types of quarks, three up-type quarks called up, charm, and top, and three down-type quarks called down, strange, and bottom. The fermions not charged under the $\mathrm{SU}(3)_{\mathrm{C}}$ are called leptons. There are six types of leptons, three charged leptons called the electron, muon, and tau, and three neutral leptons called the electron neutrino, the muon neutrino, and the tau neutrino. Finally, the scalar particle is the Higgs doublet. It initially has four degrees of freedom, but after electroweak symmetry breaking only one remains. The other three become the longitudinal modes of the massive gauge bosons. The single remaining scalar field is called the Higgs boson.

This chapter will attempt to give a concise introduction to this theory. It will start with a discussion of the representations of the Poincaré group. Possible Lagrangians for spin 0,1 , and $1 / 2$ particles are then presented. Gauge invariance, based both on abelian and non-abelian symmetry groups, will play a major role in these discussions. It will then be possible to write down the SM Lagrangian. Electroweak symmetry breaking is then considered. Finally, this chapter concludes with a discussion of the SM as an effective field theory and the origins of the hierarchy problem. This chapter draws inspiration from the textbooks by Peskin and Schroeder [5], Schwartz [6], Srednicki [7], and to a lesser extent the textbook by Weinberg [8].

\subsection{The symmetries of spacetime}

To help understand the SM, it is useful to first give a brief discussion of the symmetries of spacetime. If we have a spacetime point $x^{\mu}=(t, x, y, z)^{1}$ and we perform a Lorentz transformation characterized by the matrix $\Lambda$, then this point gets transformed into a new point $\bar{x}^{\mu}=\Lambda_{\nu}^{\mu}{ }_{\nu} x^{\nu}$. Importantly, for this to be a Lorentz transformation, we

\footnotetext{
${ }^{1}$ Throughout this thesis we set the speed of light $c=1$. We also set the fundamental unit of action $\hbar=1$.
} 
necessarily require that the interval $x^{2} \equiv x^{\mu} x_{\mu}=g_{\mu \nu} x^{\mu} x^{\nu}$ between $x^{\mu}$ and the origin be preserved, that is $x^{2}=\bar{x}^{2}$. Demanding this leads to the condition

$$
g_{\mu \nu} \Lambda_{\rho}^{\mu} \Lambda_{\sigma}^{\nu}=g_{\rho \sigma}
$$

In these expressions $g_{\mu \nu}$ is the Minkowski metric for flat spacetime. We will use the mostly minus convention for the metric so that

$$
g_{\mu \nu}=\left(\begin{array}{cccc}
+1 & 0 & 0 & 0 \\
0 & -1 & 0 & 0 \\
0 & 0 & -1 & 0 \\
0 & 0 & 0 & -1
\end{array}\right)
$$

The inverse metric is $g^{\mu \nu}$ and is equal to $g_{\mu \nu}$ for flat spacetime. Equation 2.1 and the form of the metric, equation 2.2, tell us that the Lorentz group is $\mathrm{SO}(3,1)$. This group is closely related to the group $\mathrm{SO}(4)$, which instead of preserving the metric 2.2 , simply preserves the identity matrix $\delta_{i j}$. These groups are related by Wick rotation, which is essentially the multiplication of the time component of $\operatorname{SO}(3,1)$ by the imaginary number $-i$. This correspondence is very useful. For example, the commutation relations for the generators of the Lie algebra $\operatorname{so}(n)$ are well known (see [9] for instance). Changing variables then immediately results in the commutation relations for the generators of the Lie algebra for the Lorentz group

$$
\left[\mathcal{J}^{\mu \nu}, \mathcal{J}^{\rho \sigma}\right]=i\left(g^{\nu \rho} \mathcal{J}^{\mu \sigma}-g^{\mu \rho} \mathcal{J}^{\nu \sigma}-g^{\nu \sigma} \mathcal{J}^{\mu \rho}+g^{\mu \sigma} \mathcal{J}^{\nu \rho}\right)
$$

where $\mathcal{J}^{\mu \nu}$ are the generators and satisfy $\mathcal{J}^{\mu \nu}=-\mathcal{J}^{\nu \mu}$. We can then identify the generators of rotations $J_{i}=\frac{1}{2} \epsilon_{i j k} \mathcal{J}^{j k}$ and the generators of boosts $K_{i}=\mathcal{J}^{0 i}$. These 
satisfy the commutation relations

$$
\left[J_{i}, J_{j}\right]=i \epsilon_{i j k} J_{k}, \quad\left[J_{i}, K_{j}\right]=i \epsilon_{i j k} K_{k}, \quad\left[K_{i}, K_{j}\right]=-i \epsilon_{i j k} J_{k}
$$

Finally, it is very convenient to identify the following combinations of rotation and boost generators $J_{i}^{+}=\frac{1}{2}\left(J_{i}+i K_{i}\right)$ and $J_{i}^{-}=\frac{1}{2}\left(J_{i}-i K_{i}\right)$. It is then straightforward to show that these satisfy the commutation relations

$$
\left[J_{i}^{+}, J_{j}^{+}\right]=i \epsilon_{i j k} J_{k}^{+}, \quad\left[J_{i}^{-}, J_{j}^{-}\right]=i \epsilon_{i j k} J_{k}^{-}, \quad\left[J_{i}^{+}, J_{j}^{-}\right]=0 .
$$

We see that both $J_{i}^{+}$and $J_{i}^{-}$satisfy the canonical commutation relations for the Lie algebra su(2), while at the same time commuting with each other. This means that the so $(3,1)$ Lie algebra is isomorphic to $\mathrm{su}(2) \oplus \mathrm{su}(2) .^{2}$ This allows us to characterize all finite dimensional representations of the Lorentz group by two numbers, $\left(j_{+}, j_{-}\right)$, which are half-integers. The sizes of these representations are then $\left(2 j_{+}+1\right)\left(2 j_{-}+1\right)$. Moreover, these finite dimensional representations are not unitary. In other words, if $\Lambda$ is an element of the representation, then the unitarity condition $\Lambda^{\dagger} \Lambda=\Lambda \Lambda^{\dagger}=1$ does not necessarily hold. It is also beneficial to recognize that the generators of rotations $J_{i}$ are equal to $J_{i}=J_{i}^{+}+J_{i}^{-}$. It then follows from standard addition of angular momentum rules that these representations contain angular momentums $j=j_{+}+j_{-}, j_{+}+j_{-}-1, \ldots,\left|j_{+}-j_{-}\right|+1,\left|j_{+}-j_{-}\right|$. This shows that these irreducible representations of the Lorentz group are reducible with respect to rotations. The representations which we are primarily interested in are $(0,0)=$ scalar, $\left(\frac{1}{2}, 0\right)=$ lefthanded spinor, $\left(0, \frac{1}{2}\right)=$ right-handed spinor, and $\left(\frac{1}{2}, \frac{1}{2}\right)=$ vector.

Together with the Lorentz generators, there are also the generators of time and

\footnotetext{
${ }^{2}$ This also follows from the fact that the so(4) Lie algebra is isomorphic to $\operatorname{su}(2) \oplus \mathrm{su}(2)$.
} 
space translations $P^{\mu}$. These have commutation relations amongst themselves as well as with the Lorentz generators, which are given by

$$
\left[P^{\mu}, P^{\nu}\right]=0, \quad\left[\mathcal{J}^{\mu \nu}, P^{\rho}\right]=i\left(g^{\nu \rho} P^{\mu}-g^{\mu \rho} P^{\nu}\right)
$$

These commutation relations together with those in equation 2.3 form the algebra for the Poincaré group.

\subsection{Quantum fields and their Lagrangians}

The SM describes elementary particles, and to preserve probabilities under Poincaré transformations, elementary particle states must transform under irreducible unitary representations of the Poincaré group. The representations discussed above, specified by $\left(j_{+}, j_{-}\right)$, are irreducible representations of the Poincaré group but are not unitary. In fact, there are no finite dimensional unitary representations of the Poincaré group. It is therefore necessary to determine the unitary representations of the Poincaré group. This was first done by Wigner in 1939 [10] who showed that all unitary representations are infinite dimensional and that they are classified by a non-negative real mass $m$ and a non-negative half-integer spin $j .{ }^{3}$ Moreover, if $j>0$, then there are $2 j+1$ spin states $(j, j-1, \ldots,-j+1,-j)$ for $m>0$ while there is exactly 2 spin states $(j,-j)$ if $m=0$. If $j=0$, then there is only one state for any mass.

The idea of quantum field theory is to use quantized fields to describe the dynamics of these elementary particle states which transform under the infinite dimensional unitary representations. For example, quantized fields can annihilate or create the one particle states. One reason this works is because infinite dimensional

\footnotetext{
${ }^{3}$ More precisely, these are the unitary representations that have been realized by elementary particles in Nature. There are other unitary representations which have, for example, negative or imaginary masses, but these seem to have not been realized by Nature.
} 
representations of the Poincaré group are naturally realized by fields. Moreover, fields can also transform under finite dimensional non-unitary representations of the Lorentz group, i.e. they can carry a Lorentz index. As previously described, the quantized fields are then potentially reducible with respect to spin and, at the very least, must contain the spin of the particle they are attempting to describe. If the quantum field has additional spin states, then there will be a redundancy in the description of the elementary particle, and this can lead to potential complications, for example, gauge invariance. The discussion below will highlight when this type of issue occurs.

It is useful to briefly consider how these quantized fields transform under Poincaré transformations. Consider, for example, a quantized field $\varphi_{A}(x)$ where the subscript $A$ represents a generic Lorentz index. Then under a translation where $x^{\mu} \rightarrow x^{\mu}+a^{\mu}$, the field transforms

$$
\varphi_{A}(x) \rightarrow U(a)^{-1} \varphi_{A}(x) U(a)=\varphi_{A}(x-a)=e^{i a^{\mu} \hat{P}_{\mu}} \varphi_{A}(x)
$$

so that after Taylor series expanding equation 2.7 we find the standard representation of the translation operator acting on the field

$$
\hat{P}_{\mu}=i \partial_{\mu}
$$

If we instead perform a Lorentz transformation where $x^{\mu} \rightarrow \Lambda_{\nu}^{\mu} x^{\nu}$, then the quantized field transforms

$$
\varphi_{A}(x) \rightarrow U(\Lambda)^{-1} \varphi_{A}(x) U(\Lambda)=L_{A}^{B}(\Lambda) \varphi_{B}\left(\Lambda^{-1} x\right)=e^{-\frac{i}{2} \omega^{\mu \nu} \hat{\mathcal{J}}_{\mu \nu}} \varphi_{A}(x)
$$

where $L_{A}{ }^{B}(\Lambda)$ is a matrix that depends on $\Lambda$. If we focus on the case of an infinitesimal 
transformation, then $\Lambda^{\mu}{ }_{\nu}=\delta^{\mu}{ }_{\nu}+\omega^{\mu}{ }_{\nu}$ and $L_{A}{ }^{B}(1+\omega)=\delta_{A}{ }^{B}-\frac{i}{2} \omega^{\mu \nu}\left(S_{\mu \nu}\right)_{A}{ }^{B}$. Taylor series expanding equation 2.9 then results in a representation of the Lorentz generators

$$
\hat{\mathcal{J}}_{\mu \nu}=i\left(x_{\mu} \partial_{\nu}-x_{\nu} \partial_{\mu}\right)+\left(S_{\mu \nu}\right)_{A}{ }^{B}
$$

The remainder of this section is devoted to writing down possible Lagrangians for these quantized fields. Spin 0 particles are described by a quantized real scalar field $\phi(x)$. The Lagrangian for these particles is then

$$
\mathcal{L}=\frac{1}{2} \partial^{\mu} \phi \partial_{\mu} \phi-\frac{1}{2} m^{2} \phi^{2}
$$

We can also make these particles interact by adding, for example, a term of the form $-\frac{1}{4 !} \lambda \phi^{4}$ to this Lagrangian.

Massive spin 1 particles have three spin states and are described by a quantized vector field $A_{\mu}(x)$. Notice that vector fields are reducible with respect to spin since they contain both spin 1 and spin 0 representations. At first, this may seem problematic since the particle states only have spin 1 . However, the equations of motion coming from the Proca-Lagrangian for these particles

$$
\mathcal{L}=-\frac{1}{4} F_{\mu \nu} F^{\mu \nu}+\frac{1}{2} m^{2} A_{\mu} A^{\mu}
$$

enforces the condition $\partial^{\mu} A_{\mu}=0$ and this removes the spin 0 component. In this Lagrangian $F_{\mu \nu}$ is the standard Maxwell field-strength tensor

$$
F_{\mu \nu}=\partial_{\mu} A_{\nu}-\partial_{\nu} A_{\mu}
$$

Massless spin 1 particles have only two spin states and are also described by 
a vector field $A_{\mu}(x)$, and the mismatch of two degrees of freedom leads to a few additional complications compared to the massive case. The Lagrangian is the same as in equation 2.12 except with $m \rightarrow 0$

$$
\mathcal{L}=-\frac{1}{4} F_{\mu \nu} F^{\mu \nu}
$$

This Lagrangian has a new feature not present for the massive case: gauge invariance. This is the invariance of the Lagrangian under the transformation

$$
A_{\mu}(x) \rightarrow A_{\mu}(x)+\frac{1}{e} \partial_{\mu} \alpha(x)
$$

where $e$ is a constant and $\alpha(x)$ is any spacetime dependent function. The Lagrangian is invariant under this transformation because the field-strength does not transform

$$
F_{\mu \nu} \rightarrow F_{\mu \nu}
$$

as can be easily checked by using equation 2.13. This gauge freedom allows for the removal of unphysical degrees of freedom from $A_{\mu}$.

The next step is to consider possible interaction terms between massless spin 1 particles and spin 0 particles. The key to doing so is to preserve the invariance of the Lagrangian under gauge transformations. The minimal field content capable of doing this has two mass degenerate real scalar fields, $\phi_{1}$ and $\phi_{2}$, arranged into one complex field $\phi=\frac{1}{\sqrt{2}}\left(\phi_{1}+i \phi_{2}\right)$. Ignoring the gauge field for a moment, the Lagrangian for the complex scalar field is simply $\mathcal{L}=\left(\partial^{\mu} \phi\right)^{*}\left(\partial_{\mu} \phi\right)-m^{2} \phi^{*} \phi$. If we consider a gauge transformation of the form given in equation 2.15 , then $\phi(x)$ transforms as follows

$$
\phi(x) \rightarrow e^{-i \alpha(x)} \phi(x)
$$


We see that, while the mass term in the complex scalar Lagrangian is invariant under this transformation, the kinetic term is not, and this term fails to be invariant due to the derivatives. To restore gauge invariance, we promote the derivatives to covariant derivatives, $\partial_{\mu} \rightarrow D_{\mu}=\partial_{\mu}+i e A_{\mu}$. It can then be shown that the transformation property of $D_{\mu} \phi$ under a gauge transformation is $D_{\mu} \phi \rightarrow e^{-i \alpha(x)} D_{\mu} \phi$ from which it follows that the Lagrangian

$$
\begin{aligned}
\mathcal{L} & =-\frac{1}{4} F_{\mu \nu} F^{\mu \nu}+\left(D^{\mu} \phi\right)^{*}\left(D_{\mu} \phi\right)-m^{2} \phi^{*} \phi \\
& =-\frac{1}{4} F_{\mu \nu} F^{\mu \nu}+\left(\partial^{\mu} \phi\right)^{*}\left(\partial_{\mu} \phi\right)-m^{2} \phi^{*} \phi+i e\left(\phi \partial_{\mu} \phi^{*}-\phi^{*} \partial_{\mu} \phi\right) A^{\mu}+e^{2} A_{\mu} A^{\mu} \phi^{*} \phi
\end{aligned}
$$

is gauge invariant. The last two terms on the second line of Lagrangian 2.18 are the allowed interactions, where $e$ is now recognized as a coupling constant. Thus, it is gauge invariance that tells us how spin 0 and massless spin 1 particles can interact with one another.

The invariance of the Lagrangian under these types of gauge transformations can also be described as a local U(1) symmetry. The term local refers to the fact that the transformation parameter $\alpha(x)$ is spacetime dependent. The presence of a local symmetry also implies the presence of a global symmetry, which itself implies the existence of a conserved current. There is then an associated conserved charge $Q$, which serves as the generator of the local U(1) symmetry. For the previous Lagrangian, we would say that the field $\phi$ is charged under the gauge symmetry with charge -1 . More generally, the field $\phi$ can carry charge $q$, in which case it would transform under a gauge transformation as $\phi(x) \rightarrow e^{i q \alpha(x)} \phi(x)$ and the covariant derivative would be modified to $D_{\mu}=\partial_{\mu}-i q e A_{\mu}$. Gauge symmetries can also be based on other symmetry groups and this leads to a few additional complications. These types of non-abelian gauge symmetries are discussed in more detail in section 2.4. 
Regardless of their mass, spin one-half particles have two spin states, and can be described by a quantum field which transforms as a left-handed Weyl spinor (the $\left(\frac{1}{2}, 0\right)$ representation) or as a right-handed Weyl spinor (the $\left(0, \frac{1}{2}\right)$ representation). As a matter of fact, the rotation and boost generators for the $\left(\frac{1}{2}, 0\right)$ representation and the rotation and boost generators for the $\left(0, \frac{1}{2}\right)$ representation are adjoints of each other. Because of this, we can always make a left-handed spinor right-handed or a right-handed spinor left-handed by taking a hermitian conjugate. For example, if $\chi_{\alpha}$ is a left-handed spinor, then $\left(\chi_{\alpha}\right)^{\dagger} \equiv \chi_{\dot{\alpha}}^{\dagger}$ is a right-handed spinor. Here we have used the van der Waerden notation: undotted indices represent left-handed spinors and dotted indices (with hermitian conjugate symbols) represent right-handed spinors. We can also raise and lower these indices with the anti-symmetric tensors $\epsilon_{\alpha \beta}, \epsilon^{\alpha \beta}$, $\epsilon_{\dot{\alpha} \dot{\beta}}, \epsilon^{\dot{\alpha} \dot{\beta}}$ with $\epsilon_{21}=-\epsilon_{12}=\epsilon^{12}=-\epsilon^{21}=1$ for both dotted and undotted $\epsilon$ tensors. When doing so, it is important to remember to always contract the second index.

To form Lorentz invariant quantities, we contract a raised undotted index with a lowered undotted index $\left(\chi^{\alpha} \psi_{\alpha}\right)$ or a lowered dotted index with a raised dotted index $\left(\chi_{\dot{\alpha}}^{\dagger} \psi^{\dagger \dot{\alpha}}\right)$. The order of the contracted indices is to help avoid minus sign errors. As an example,

$$
\chi \psi \equiv \chi^{\alpha} \psi_{\alpha}=\epsilon^{\alpha \beta} \chi_{\beta} \psi_{\alpha}=-\epsilon^{\alpha \beta} \psi_{\alpha} \chi_{\beta}=+\epsilon^{\beta \alpha} \psi_{\alpha} \chi_{\beta}=\psi^{\beta} \chi_{\beta}=\psi \chi
$$

and similarly $\chi^{\dagger} \psi^{\dagger} \equiv \chi_{\dot{\alpha}}^{\dagger} \psi^{\dagger \dot{\alpha}}=\psi_{\dot{\alpha}}^{\dagger} \chi^{\dagger \dot{\alpha}}=\psi^{\dagger} \chi^{\dagger}$. Here we used the fact that since these spinors will represent fermions they must anticommute.

We will mainly use left-handed spinors to describe the dynamics of spin onehalf particles. This is the standard choice made in supersymmetry which will be 
introduced in chapter 3. The Lagrangian for these particles is then

$$
\mathcal{L}=i \chi_{\dot{\alpha}}^{\dagger} \bar{\sigma}^{\mu \dot{\alpha} \beta} \partial_{\mu} \chi_{\beta}-\frac{1}{2} m\left(\chi^{\alpha} \chi_{\alpha}+\chi_{\dot{\alpha}}^{\dagger} \chi^{\dagger \dot{\alpha}}\right)
$$

where we show the index contractions for clarity. Here $\bar{\sigma}^{\mu \dot{\alpha} \beta}=\left(I,-\sigma_{1},-\sigma_{2},-\sigma_{3}\right)$ where $I$ is the two dimensional identity matrix and the $\sigma_{i}$ are the Pauli matrices

$$
\sigma_{1}=\left(\begin{array}{cc}
0 & 1 \\
1 & 0
\end{array}\right) \quad \sigma_{2}=\left(\begin{array}{cc}
0 & -i \\
i & 0
\end{array}\right) \quad \sigma_{3}=\left(\begin{array}{cc}
1 & 0 \\
0 & -1
\end{array}\right)
$$

Another useful set of matrices are $\sigma_{\alpha \dot{\beta}}^{\mu}=\left(I, \sigma_{1}, \sigma_{2}, \sigma_{3}\right)$ which appear in the kinetic term for the fermion if we had used a right-handed spinor. The mass term in Lagrangian 2.20 is referred to as a Majorana mass term.

It is possible to rewrite this Lagrangian in four-component spinor notation. First, introduce the Majorana spinor

$$
\Psi=\left(\begin{array}{c}
\chi_{\alpha} \\
\chi^{\dagger \dot{\alpha}}
\end{array}\right)
$$

and the $\beta$ and Dirac $\gamma$-matrices

$$
\beta=\left(\begin{array}{cc}
0 & \delta^{\dot{\alpha}}{ }_{\dot{\beta}} \\
\delta_{\alpha}{ }^{\beta} & 0
\end{array}\right) \quad \text { and } \quad \gamma^{\mu}=\left(\begin{array}{cc}
0 & \sigma_{\alpha \dot{\beta}}^{\mu} \\
\bar{\sigma}^{\mu \dot{\alpha} \beta} & 0
\end{array}\right)
$$

Numerically $\beta=\gamma^{0}$ but they have different index structures and so we identify them as different matrices. The Lagrangian in equation 2.20 can then be rewritten as

$$
\mathcal{L}=\frac{i}{2} \bar{\Psi} \gamma^{\mu} \partial_{\mu} \Psi-\frac{1}{2} m \bar{\Psi} \Psi
$$


where $\bar{\Psi} \equiv \Psi^{\dagger} \beta$.

It is very often the case that a Majorana mass term for a fermion $\chi$ is forbidden because it transforms non-trivially under a symmetry. However, a Dirac mass term for the fermion can be written down provided there is a second spinor $\psi$ which transforms in the conjugate representation of the symmetry. The Lagrangian is then

$$
\mathcal{L}=i \chi^{\dagger} \bar{\sigma}^{\mu} \partial_{\mu} \chi+i \psi^{\dagger} \bar{\sigma}^{\mu} \partial_{\mu} \psi-m\left(\chi \psi+\chi^{\dagger} \psi^{\dagger}\right)
$$

We can rewrite this Lagrangian by introducing a Dirac spinor ${ }^{4}$

$$
\Psi=\left(\begin{array}{c}
\chi_{\alpha} \\
\psi^{\dagger \dot{\alpha}}
\end{array}\right)
$$

The Lagrangian 2.25 can then be expressed

$$
\mathcal{L}=i \bar{\Psi} \gamma^{\mu} \partial_{\mu} \Psi-m \bar{\Psi} \Psi
$$

Dirac spinors are perhaps the best-known way to represent fermionic fields. This is possibly due to the fact that in the SM all mass terms (except potentially the mass terms for the neutrinos) are of the Dirac type.

In the SM, it is generally more standard to work with four-component spinor notation than two-component spinor notation. However, there are times where we would like to project out the left- or right-handed components of a Dirac or Majorana

\footnotetext{
${ }^{4}$ Perhaps unfortunately, the standard notation is to use $\Psi$ for both Majorana and Dirac spinors.
} 
spinor. To accomplish this, consider the fifth Dirac $\gamma$-matrix

$$
\gamma^{5}=i \gamma^{0} \gamma^{1} \gamma^{2} \gamma^{3}=\left(\begin{array}{cc}
-\delta_{\alpha}^{\beta} & 0 \\
0 & +\delta_{\dot{\beta}}^{\dot{\alpha}}
\end{array}\right)
$$

We can then form the left and right projection operators

$$
P_{L}=\frac{1}{2}\left(1-\gamma^{5}\right)=\left(\begin{array}{cc}
\delta_{\alpha}^{\beta} & 0 \\
0 & 0
\end{array}\right) \quad \text { and } \quad P_{R}=\frac{1}{2}\left(1+\gamma^{5}\right)=\left(\begin{array}{cc}
0 & 0 \\
0 & \delta_{\dot{\beta}}^{\dot{\alpha}}
\end{array}\right)
$$

so that when these act on a Dirac or Majorana spinor, they project out the left- and right-handed spinors, respectively.

Next, consider how spin one-half particles can interact with spin 0 or massless spin 1 particles. Interactions between spin one-half and spin 0 particles are called Yukawa interactions. An example Yukawa interaction is

$$
\mathcal{L}=-y \phi \bar{\Psi} \Psi
$$

where $\phi$ is a real scalar field and $\Psi$ can be either a Majorana or Dirac spinor.

Interactions between spin one-half particles and massless spin 1 particles must respect gauge invariance. Consider a single fermion described by a left-handed spinor $\chi$ charged under a U(1) gauge symmetry. Under the gauge symmetry, this field transforms $\chi(x) \rightarrow e^{-i \alpha(x)} \chi(x)$ where we let this field have charge -1 (cf. equation 2.17). We see that the Majorana mass term in Lagrangian 2.20 is now forbidden. Additionally, to make the kinetic term invariant under this transformation we again 
promote the derivative to a gauge covariant derivative. The Lagrangian is then

$$
\mathcal{L}=-\frac{1}{4} F_{\mu \nu} F^{\mu \nu}+i \chi^{\dagger} \bar{\sigma}^{\mu} D_{\mu} \chi
$$

with $D_{\mu}=\partial_{\mu}+i e A_{\mu}$ as before. However, it turns out that this Lagrangian is fatally flawed as it will suffer from a gauge anomaly. An anomaly is a symmetry that exists at the classical level but is broken at the quantum level. In this case, radiative corrections in the form of loop diagrams will break the gauge symmetry and this will do violence to the theory. The reason why this occurs is because Lagrangian 2.31 contains only a single fermion. To render the theory anomaly free, it is sufficient to introduce another left-handed spinor field $\psi$ and make it transform under the gauge symmetry with opposite charge to that of $\chi$, i.e. $\psi(x) \rightarrow e^{+i \alpha(x)} \psi(x)$. An anomaly free Lagrangian is then

$$
\begin{aligned}
\mathcal{L} & =-\frac{1}{4} F_{\mu \nu} F^{\mu \nu}+i \chi^{\dagger} \bar{\sigma}^{\mu} D_{\mu} \chi+i \psi^{\dagger} \bar{\sigma}^{\mu} D_{\mu} \psi-m\left(\chi \psi+\chi^{\dagger} \psi^{\dagger}\right) \\
& =-\frac{1}{4} F_{\mu \nu} F^{\mu \nu}+i \bar{\Psi} \gamma^{\mu} D_{\mu} \Psi-m \bar{\Psi} \Psi \\
& =-\frac{1}{4} F_{\mu \nu} F^{\mu \nu}+i \bar{\Psi} \gamma^{\mu} \partial_{\mu} \Psi-m \bar{\Psi} \Psi-e \bar{\Psi} \gamma^{\mu} \Psi A_{\mu}
\end{aligned}
$$

where $^{5} D_{\mu} \psi=\left(\partial_{\mu}-i e A_{\mu}\right) \psi$ and we have introduced a possible gauge invariant Dirac mass term for $\chi$ and $\psi$. In the second line we switched to more standard Dirac notation with the Dirac spinor being the same as that in equation 2.26 and in the third line we expanded the covariant derivative to show the allowed interaction between the Dirac fermion and the massless gauge field.

A fermion charged under a gauge symmetry which does not have a partner

\footnotetext{
${ }^{5}$ We will use the symbol $D_{\mu}$ to represent every covariant derivative. The exact form of the covariant derivative then depends on the gauge charges of the quantum field that it is acting on.
} 
fermion charged under the conjugate representation is called a chiral fermion. The fermion $\chi$ in Lagrangian 2.31 is an example of a chiral fermion. On the other hand, if a fermion does have a partner fermion charged under the conjugate representation, then the pair are called a vector fermion. For example, $\chi$ and $\psi$ in Lagrangian 2.32 form a vector fermion described by the Dirac spinor $\Psi$. While a single chiral fermion led to a gauge anomaly for Lagrangian 2.31, it is possible to have anomaly free gauge theories with chiral fermions (the SM being one example). In these theories, all potential gauge anomalies cancel when summing over the effects of all chiral fermions. Importantly, mass terms for chiral fermions are always forbidden by the gauge symmetry. In contrast, mass terms for vector fermions are always allowed.

\subsection{Non-abelian gauge theories}

In the previous section, gauge invariance was based on a local U(1) symmetry. However, as mentioned, gauge symmetries can also be based on non-abelian symmetry groups. These symmetry groups can be of the form $\mathrm{SU}(n), \mathrm{SO}(n), \mathrm{Sp}(n)$, or the groups generated by the five exceptional Lie algebras.

For gauge theories based on non-abelian symmetry groups, the number of gauge fields is equal to the number of symmetry generators. If there are $n$ generators, then there are $n$ gauge fields which we denote by $A_{\mu}^{a}$. The field-strengths for these gauge fields are similar to the abelian case, equation 2.13, but have the following modification

$$
F_{\mu \nu}^{a}=\partial_{\mu} A_{\nu}^{a}-\partial_{\nu} A_{\mu}^{a}+g f^{a b c} A_{\mu}^{b} A_{\nu}^{c}
$$

where $g$ is the coupling constant for the gauge symmetry and $f^{a b c}$ are the structure constants for the group. The U(1) symmetry group does not have structure constants 
and so we can recover equation 2.13 from equation 2.33 by setting $f^{a b c} \rightarrow 0$. Under a gauge transformation characterized by $n$ scalar fields $\alpha^{a}(x)$, the gauge fields and the field-strengths have the following infinitesimal transformation laws

$$
A_{\mu}^{a} \rightarrow A_{\mu}^{a}+\frac{1}{g} \partial_{\mu} \alpha^{a}-f^{a b c} \alpha^{b} A_{\mu}^{c} \quad \text { and } \quad F_{\mu \nu}^{a} \rightarrow F_{\mu \nu}^{a}-f^{a b c} \alpha^{b} F_{\mu \nu}^{c}
$$

which generalizes equations 2.15 and 2.16. We see that the field-strengths are no longer gauge invariant but instead transform in the adjoint representation of the gauge group. This explains why the number of gauge fields matches the number of symmetry generators. The Lagrangian for these gauge fields is then

$$
\mathcal{L}=-\frac{1}{4} F_{\mu \nu}^{a} F^{a \mu \nu}
$$

where the sum over the repeated index $a$ is crucial for achieving invariance under a gauge transformation.

We can also have scalars and fermions charged under non-abelian gauge symmetries. In this case, these particles transform under some representation of the gauge group. For example, consider a theory which has both complex scalars $\phi$ and fermions $\chi$ transforming under representations of the gauge group generated by generators $t_{r_{\phi}}^{a}$ and $t_{r_{\chi}}^{a}$, respectively. Under a gauge transformation, these fields transform as

$$
\phi \rightarrow e^{i \alpha^{a} t_{r_{\phi}}^{a} \phi} \quad \text { and } \quad \chi \rightarrow e^{i \alpha^{a} t_{r \chi}^{a}} \chi
$$

Here $\phi$ and $\chi$ are vectors of scalar and fermionic fields whose sizes match the size of the representations that they individually transform under. If both representations 
are complex, ${ }^{6}$ then the Lagrangian for this theory is

$$
\mathcal{L}=-\frac{1}{4} F_{\mu \nu}^{a} F^{a \mu \nu}+\left(D_{\mu} \phi\right)^{\dagger}\left(D^{\mu} \phi\right)-m^{2} \phi^{\dagger} \phi+i \chi^{\dagger} \bar{\sigma}^{\mu} D_{\mu} \chi
$$

where $D_{\mu} \phi=\left(\partial_{\mu}-i g A_{\mu}^{a} t_{r_{\phi}}^{a}\right) \phi$ and $D_{\mu} \chi=\left(\partial_{\mu}-i g A_{\mu}^{a} t_{r_{\chi}}^{a}\right) \chi$. This Lagrangian will again suffer from a gauge anomaly due to the chiral fermions. Without changing the symmetry group, this can be solved two different ways. The first is to introduce another set of fermions $\psi$ transforming in the conjugate representation so that $\chi$ and $\psi$ form a vector fermion. The fermionic part of the Lagrangian can then be naturally written in Dirac notation such as in Lagrangian 2.32. The second is to change the representation generated by $t_{r_{\chi}}^{a}$ and insist for it to be real. In fact, a Majorana mass term for the fermions can then be written down. For example, if $\chi$ were to transform in the adjoint representation, then we can include the term $-\frac{1}{2} m \chi^{a} \chi^{a}+$ h.c. to the Lagrangian. These types of terms, while not relevant for the SM, do appear in many SM extensions including supersymmetry.

\subsection{Field content of the Standard Model}

The $\mathrm{SM}$ is a gauge theory based on the gauge group $\mathrm{SU}(3)_{\mathrm{C}} \times \mathrm{SU}(2)_{\mathrm{L}} \times \mathrm{U}(1)_{\mathrm{Y}}$. As will be discussed in section 2.6, this gauge group is spontaneously broken down to the group $\mathrm{SU}(3)_{\mathrm{C}} \times \mathrm{U}(1)_{\mathrm{EM}}$ but we ignore this feature for the moment.

The symmetry group $\mathrm{SU}(n)$ has $n^{2}-1$ generators. It then follows that there are eight gauge fields for $\mathrm{SU}(3)_{\mathrm{C}}$ and three gauge fields for $\mathrm{SU}(2)_{\mathrm{L}}$. As usual, there is a single gauge field for $\mathrm{U}(1)_{\mathrm{Y}}$. These gauge fields are denoted $G_{\mu}^{a}, W_{\mu}^{b}$, and $B_{\mu}$,

\footnotetext{
${ }^{6} \mathrm{~A}$ representation generated by generators $t_{r}^{a}$ is complex if it is inequivalent to its conjugate representation generated by generators $-\left(t_{r}^{a}\right)^{*}$. The fundamental and anti-fundamental representations of $\mathrm{SU}(n)$ with $n \geq 3$ are examples of complex representations. If the representation is not complex, then it is real. The adjoint representations of any symmetry groups are examples of real representations.
} 
respectively, with $a=1, \ldots, 8$ and $b=1,2,3$. The associated field-strength tensors are denoted $G_{\mu \nu}^{a}, W_{\mu \nu}^{b}$, and $B_{\mu \nu}$. The forms of the field-strengths $G_{\mu \nu}^{a}$ and $W_{\mu \nu}^{b}$ are the same as in equation 2.33 while the form of $B_{\mu \nu}$ is the same as in equation 2.13. The $\mathrm{SU}(3)_{\mathrm{C}}, \mathrm{SU}(2)_{\mathrm{L}}$, and $\mathrm{U}(1)_{\mathrm{Y}}$ gauge couplings are denoted $g_{s}, g$, and $g^{\prime}$, respectively. The pure gauge part of the SM Lagrangian is given by ${ }^{7}$

$$
\mathcal{L}_{\text {gauge }}^{\mathrm{SM}}=-\frac{1}{4} G_{\mu \nu}^{a} G^{a \mu \nu}-\frac{1}{4} W_{\mu \nu}^{b} W^{b \mu \nu}-\frac{1}{4} B_{\mu \nu} B^{\mu \nu}
$$

All fermions in the SM are chiral. ${ }^{8}$ We use the notation $\left(\boldsymbol{r}_{\mathbf{C}}, \boldsymbol{r}_{\mathbf{L}}, Y\right)$ to denote a fermion transforming in the $\boldsymbol{r}_{\mathbf{C}}$ representation of $\mathrm{SU}(3)_{\mathrm{C}}, \boldsymbol{r}_{\mathbf{L}}$ representation of $\mathrm{SU}(2)_{\mathrm{L}}$, and with hypercharge $Y$. The fermions in the SM are then: ${ }^{9}$

- a left-handed spinor $Q^{i}$ with gauge charges $(3,2,1 / 6)$,

- a right-handed spinor $U^{i}$ with gauge charges $(\mathbf{3}, \mathbf{1}, 2 / 3)$,

- a right-handed spinor $D^{i}$ with gauge charges $(\mathbf{3}, \mathbf{1},-1 / 3)$,

\footnotetext{
${ }^{7}$ In fact, there are other pure gauge terms which we have ignored up to this point that can be written down. These are

$$
\mathcal{L}_{\theta}^{\mathrm{SM}}=\frac{\theta_{\mathrm{C}} g_{s}^{2}}{64 \pi^{2}} \epsilon^{\mu \nu \rho \sigma} G_{\mu \nu}^{a} G_{\rho \sigma}^{a}+\frac{\theta_{\mathrm{L}} g^{2}}{64 \pi^{2}} \epsilon^{\mu \nu \rho \sigma} W_{\mu \nu}^{b} W_{\rho \sigma}^{b}+\frac{\theta_{\mathrm{Y}} g^{2}}{64 \pi^{2}} \epsilon^{\mu \nu \rho \sigma} B_{\mu \nu} B_{\rho \sigma}
$$

where the $\theta_{i}$ are the so-called vacuum angles. These terms are closely related to anomalous transformations of chiral fermions in that they can be generated by performing transformations on chiral fermions charged under these gauge symmetries. Moreover, it can be shown that each of these terms are total derivatives and therefore do not contribute to calculations based on perturbation theory. However, these terms can have significant non-perturbative topological effects. For example, a nonzero QCD vacuum angle $\theta_{\mathrm{C}}$ generates an electric dipole moment for the neutron. While one would expect $\theta_{\mathrm{C}}$ to be of order one, current measurements of the electric dipole moment for the neutron imply $\theta_{\mathrm{C}}<10^{-10}$. The smallness of $\theta_{\mathrm{C}}$ is commonly referred to as the strong CP problem. Further, the $\mathrm{SU}(2)_{\mathrm{L}}$ vacuum angle $\theta_{\mathrm{L}}$ is unphysical as it can be set to zero without any consequences by performing chiral rotations. Finally, the $\mathrm{U}(1)_{\mathrm{Y}}$ vacuum angle $\theta_{\mathrm{Y}}$ is also unphysical for topological reasons. See [11] for additional discussion regarding these types of terms.

${ }^{8}$ This fact is somewhat unsurprising. In the limit of unbroken gauge symmetries, chiral fermions are necessarily massless. On the other hand, mass terms for vector fermions are always allowed and therefore these particles can be naturally heavy.

${ }^{9}$ Although $U^{i}, D^{i}$, and $E^{i}$ are right-handed spinors, we do not put hermitian conjugates on these fields in an attempt to ease the notation.
} 
- a left-handed spinor $L^{i}$ with gauge charges $(\mathbf{1}, \mathbf{2},-1 / 2)$,

- a right-handed spinor $E^{i}$ with gauge charges $(\mathbf{1}, \mathbf{1},-1)$.

The index $i$ on each of these spinors represents a generation index. There are three generations for each fermion. An interesting fact about the fermions of the SM is that an individual generation of these fields is precisely the smallest chiral fermionic content capable of producing an anomaly free gauge theory. The SM Lagrangian is normally written in four-component spinor notation. To do so, we insert each of these two-component spinors into a four-component spinor then act on the fourcomponent spinor with an appropriate projection operator. For example, we can write $Q_{L}^{i}=P_{L} \Psi$ where $\Psi$ is a four-component spinor whose left-handed component is $Q^{i}$. Similarly, we write $u_{R}=P_{R} \Psi$ where $\Psi$ is now a four-component spinor whose right-handed component is $U^{i}$. Continuing, we let $d_{R}^{i}, L_{L}^{i}$, and $e_{R}^{i}$ represent the results of applying this procedure on $D^{i}, L^{i}$, and $E^{i}$, respectively. The fermionic part of the SM Lagrangian is then

$$
\mathcal{L}_{\text {fermions }}^{\mathrm{SM}}=\sum_{\Psi=Q_{L}, u_{R}, d_{R}, L_{L}, e_{R}} i \bar{\Psi}^{i} \gamma^{\mu} D_{\mu} \Psi^{i}
$$

where

$$
D_{\mu}=\partial_{\mu}-i g_{s} G_{\mu}^{a} t_{r_{\mathrm{C}}}^{a}-i g W_{\mu}^{b} t_{r_{\mathrm{L}}}^{b}-i g^{\prime} Y B_{\mu}
$$

is the covariant derivative which is determined by the gauge charges. For example, the covariant derivative for the field $Q_{L}$ is $D_{\mu}=\partial_{\mu}-i g_{s} G_{\mu}^{a} \frac{\lambda^{a}}{2}-i g W_{\mu}^{b} \frac{\sigma^{b}}{2}-i \frac{g^{\prime}}{6} B_{\mu}$ where $\lambda^{a}$ and $\sigma^{b}$ are the Gell-Mann and Pauli matrices, respectively.

The scalar content of the SM consists of a single Higgs doublet $H$ with gauge 
charges $(\mathbf{1}, \mathbf{2}, 1 / 2)$. Its Lagrangian is

$$
\mathcal{L}_{\mathrm{Higgs}}^{\mathrm{SM}}=\left(D_{\mu} H\right)^{\dagger}\left(D^{\mu} H\right)-V_{\mathrm{Higgs}}^{\mathrm{SM}}
$$

where the covariant derivative acting on the Higgs doublet is $D_{\mu}=\partial_{\mu}-i g W_{\mu}^{b} \frac{\sigma^{b}}{2}-i \frac{g^{\prime}}{2} B_{\mu}$ and

$$
V_{\mathrm{Higgs}}^{\mathrm{SM}}=\mu^{2} H^{\dagger} H+\lambda\left(H^{\dagger} H\right)^{2}
$$

is the scalar potential which plays a vital role in electroweak symmetry breaking. Finally, there are also gauge invariant Yukawa interactions between $H$ and the fermions. These are

$$
\mathcal{L}_{\text {Yukawa }}^{\mathrm{SM}}=-y_{u}^{i j} \epsilon^{a b} \bar{Q}_{L a}^{i} H_{b}^{\dagger} u_{R}^{j}-y_{d}^{i j} \bar{Q}_{L}^{i} H d_{R}^{j}-y_{e}^{i j} \bar{L}_{L}^{i} H e_{R}^{j}+\text { h.c. }
$$

where the $\epsilon^{a b}$ tensor contracts two SU $(2)_{\mathrm{L}} \overline{\mathbf{2}}$ indices to enforce gauge invariance. The entire SM Lagrangian is then

$$
\mathcal{L}^{\mathrm{SM}}=\mathcal{L}_{\text {gauge }}^{\mathrm{SM}}+\mathcal{L}_{\text {fermions }}^{\mathrm{SM}}+\mathcal{L}_{\text {Higgs }}^{\mathrm{SM}}+\mathcal{L}_{\text {Yukawa }}^{\mathrm{SM}}
$$

\subsection{Electroweak symmetry breaking}

The scalar potential in the $\mathrm{SM}$ is given by $V_{\mathrm{Higgs}}^{\mathrm{SM}}$ defined in equation 2.43 . If the mass squared term $\mu^{2}$ is positive, then minimizing the scalar potential results in zero vacuum expectation value (vev) for the Higgs. The vacuum is then left invariant under all $\mathrm{SU}(2)_{\mathrm{L}} \times \mathrm{U}(1)_{\mathrm{Y}}$ gauge transformations and the gauge bosons and fermions 
remain massless. ${ }^{10}$ This is phenomenologically unacceptable and we therefore conclude that $\mu^{2}$ must be negative. Minimizing the scalar potential then results in the Higgs acquiring a vev given by $\langle H\rangle=\sqrt{\frac{-\mu^{2}}{2 \lambda}} \equiv v \cdot{ }^{11}$ Under a $\mathrm{SU}(2)_{\mathrm{L}} \times \mathrm{U}(1)_{\mathrm{Y}}$ gauge transformation characterized by scalar functions $\alpha^{a}(x)$ and $\beta(x)$, the Higgs transforms $H \rightarrow e^{i \alpha^{a} \sigma^{a} / 2} e^{i \beta / 2} H$. This gauge freedom allows us to take $v$ to be real and positive and in the lower component of $H$

$$
\langle H\rangle=\left(\begin{array}{l}
0 \\
v
\end{array}\right)
$$

The presence of a vev in this component of the Higgs field breaks the invariance of the vacuum under most $\mathrm{SU}(2)_{\mathrm{L}} \times \mathrm{U}(1)_{\mathrm{Y}}$ gauge transformations. For example, suppose we perform a hypercharge gauge transformation characterized by $\beta$. Then

$$
e^{i \beta / 2}\langle H\rangle=\left(\begin{array}{cc}
e^{i \beta / 2} & 0 \\
0 & e^{i \beta / 2}
\end{array}\right)\left(\begin{array}{l}
0 \\
v
\end{array}\right)=\left(\begin{array}{c}
0 \\
v e^{i \beta / 2}
\end{array}\right) \neq\langle H\rangle
$$

which signifies that the hypercharge symmetry has been broken. However, there is one linear combination of symmetry generators which remains unbroken. For a gauge transformation with $\alpha^{1}=\alpha^{2}=0$ and $\alpha^{3}=\beta$, we have that $e^{i \alpha^{a} \sigma^{a} / 2} e^{i \beta / 2}=e^{i \operatorname{diag}(\beta, 0)}=$ $\operatorname{diag}\left(e^{i \beta}, 1\right)$ which leaves $\langle H\rangle$ invariant. The single unbroken symmetry generator is then

$$
Q=T^{3}+Y
$$

\footnotetext{
${ }^{10}$ This statement ignores strong colour dynamics, which would cause the vacuum to spontaneously break the electroweak symmetry after confinement, which in turn would generate tiny masses for some SM particles. For example, the $W$ bosons would eat the now massless Goldstone pions and acquire a mass $m_{W}=g f_{\pi} / 2 \approx 29 \mathrm{MeV}[12]$.

${ }^{11}$ We will use the symbol $v$ to represent the vev of the Higgs doublet. Another very common notation is to let $v$ represent the vev of the Higgs boson, in which case it is a factor of $\sqrt{2}$ larger.
} 
where $T^{3}$ is the third generator of the $\mathrm{SU}(2)_{\mathrm{L}}$ symmetry. This symmetry generator is identified with electric charge and the gauge group it generates is $\mathrm{U}(1)_{\mathrm{EM}}$. For example, the $e_{R}^{i}$ fields are $\mathrm{SU}(2)_{\mathrm{L}}$ singlets and therefore have electric charge $Q=Y=$ -1 as expected. Thus, we see that while the Lagrangian of the SM is invariant under $\mathrm{SU}(3)_{\mathrm{C}} \times \mathrm{SU}(2)_{\mathrm{L}} \times \mathrm{U}(1)_{\mathrm{Y}}$ gauge transformations, the ground state of the vacuum is only invariant under gauge transformations of the subgroup $\mathrm{SU}(3)_{\mathrm{C}} \times \mathrm{U}(1)_{\mathrm{EM}}$.

There are different ways in which the Higgs field can be parameterized. One way is to write

$$
H=\left(\begin{array}{c}
G^{+} \\
\frac{1}{\sqrt{2}}\left(h+i A^{0}\right)+v
\end{array}\right)
$$

where $h$ and $A^{0}$ are respectively $\mathrm{CP}$-even and CP-odd real scalars and $G^{+}$is a positively charged complex scalar (whose complex conjugate is denoted $G^{-}$). The scalar $h$ is the Higgs boson and is a physical degree of freedom. On the other hand, the scalars $A^{0}, G^{+}$, and $G^{-}$are unphysical: they are the Goldstone bosons of the spontaneously broken gauge symmetry and become the longitudinal modes for the now massive gauge bosons. A simple way to see that these fields are unphysical is to consider another parameterization of the Higgs field

$$
H=U(x)\left(\begin{array}{c}
0 \\
\frac{1}{\sqrt{2}} h+v
\end{array}\right)
$$

where $U(x)$ represents a general $\mathrm{SU}(2)_{\mathrm{L}}$ gauge transformation. We can now make a gauge transformation to remove the matrix $U(x)$. This choice of gauge is known as unitarity gauge.

To determine the masses of the gauge bosons it is sufficient to sub in the vev 
of the Higgs, equation 2.46, into its kinetic term. This results in the following terms in the Lagrangian

$$
\Delta \mathcal{L}=\frac{v^{2}}{4}\left[g^{2}\left(W_{\mu}^{1}\right)^{2}+g^{2}\left(W_{\mu}^{2}\right)^{2}+\left(-g W_{\mu}^{3}+g^{\prime} B_{\mu}\right)^{2}\right]
$$

Comparing with the Proca-Lagrangian given in equation 2.12, these terms imply the existence of the massive $W$ bosons

$$
W_{\mu}^{ \pm}=\frac{1}{\sqrt{2}}\left(W_{\mu}^{1} \mp i W_{\mu}^{2}\right) \quad \text { with mass } \quad m_{W}=\frac{g v}{\sqrt{2}}
$$

and the massive $Z$ boson

$$
Z_{\mu}=\cos \theta_{W} W_{\mu}^{3}-\sin \theta_{W} B_{\mu} \quad \text { with mass } \quad m_{Z}=\sqrt{\frac{g^{2}+g^{\prime 2}}{2}} v
$$

where $\tan \theta_{W}=g^{\prime} / g$. The orthogonal combination of $W_{\mu}^{3}$ and $B_{\mu}$ fields remains massless

$$
A_{\mu}=\sin \theta_{W} W_{\mu}^{3}+\cos \theta_{W} B_{\mu} \quad \text { with mass } \quad m_{A}=0
$$

and is identified as the vector boson for the unbroken $\mathrm{U}(1)_{\mathrm{EM}}$ symmetry, i.e. the photon. The electromagnetic coupling constant $e$ is determined by $g$ and $g^{\prime}$ and is given by

$$
e=\frac{g g^{\prime}}{\sqrt{g^{2}+g^{\prime 2}}}
$$

After the $\mathrm{SU}(2)_{\mathrm{L}} \times \mathrm{U}(1)_{\mathrm{Y}}$ gauge symmetry is broken to $\mathrm{U}(1)_{\mathrm{EM}}$, it is natural to distinguish the different components of the fermionic $\mathrm{SU}(2)_{\mathrm{L}}$ doublets. With this in 
mind, we write

$$
Q_{L}^{i}=\left(\begin{array}{c}
u_{L}^{i} \\
d_{L}^{i}
\end{array}\right) \quad \text { and } \quad L_{L}^{i}=\left(\begin{array}{c}
\nu_{L}^{i} \\
e_{L}^{i}
\end{array}\right)
$$

We can then sub in the vev of the Higgs, equation 2.46, into the SM Yukawa interaction terms, equation 2.44, to determine the masses generated for the SM fermions. Doing so results in the following terms in the Lagrangian

$$
\Delta \mathcal{L}=-y_{u}^{i j} v \bar{u}_{L}^{i} u_{R}^{j}-y_{d}^{i j} v \bar{d}_{L}^{i} d_{R}^{j}-y_{e}^{i j} v \bar{e}_{L}^{i} e_{R}^{j}+\text { h.c. }
$$

The next step is to diagonalize the Yukawa couplings. This can be accomplished by introducing unitary rotation matrices $U_{k}$ and $W_{k}(k=u, d, e)$ so that $y_{k}=U_{k} D_{k} W_{k}^{\dagger}$ where $D_{k}$ is a diagonal matrix with positive elements. Finally, by using the $U$ matrices to rotate the left-handed fields and the $W$ matrices to rotate the right-handed fields (for example $u_{L}^{i} \rightarrow U_{u}^{i j} u_{L}^{j}$ and $u_{R}^{i} \rightarrow W_{u}^{i j} u_{R}^{j}$ ), the Lagrangian can be rewritten as

$$
\Delta \mathcal{L}=-m_{u}^{i} \bar{u}_{L}^{i} u_{R}^{i}-m_{d}^{i} \bar{d}_{L}^{i} d_{R}^{i}-m_{e}^{i} \bar{e}_{L}^{i} e_{R}^{i}+\text { h.c. }
$$

where $m_{k}^{i}=D_{k}^{i i} v$ are the masses of the fermions generated from electroweak symmetry breaking.

The rotation matrices $U$ and $W$ mostly cancel out of the kinetic terms for the SM fermions, equation 2.40. However, one place where they do not is the kinetic term for the $Q_{L}$ field. This is because there are interactions mediated by the charged $W$ bosons connecting the two components of the $Q_{L}$ field, $u_{L}$ and $d_{L}$, and that the rotation matrices for these fields, $U_{u}$ and $U_{d}$, are independent of each other. Focusing 
on this term in the Lagrangian, we find

$$
\mathcal{L}^{\mathrm{SM}} \supset \frac{g}{\sqrt{2}} \bar{u}_{L}^{i} \gamma^{\mu} d_{L}^{i} W_{\mu}^{+} \rightarrow \frac{g}{\sqrt{2}}\left(U_{u}^{\dagger} U_{d}\right)^{i j} \bar{u}_{L}^{i} \gamma^{\mu} d_{L}^{j} W_{\mu}^{+}=\frac{g}{\sqrt{2}} V^{i j} \bar{u}_{L}^{i} \gamma^{\mu} d_{L}^{j} W_{\mu}^{+}
$$

where $V=U_{u}^{\dagger} U_{d}$ is the Cabibbo-Kobayashi-Maskawa (CKM) matrix. The CKM matrix is a three dimensional unitary matrix which implies it can initially be parameterized by three angles and six complex phases. However, chiral rotations of the six fermions $u_{L}^{i}$ and $d_{L}^{i}$ allows for the removal of five of these phases (an overall phase cannot be removed). The final form of the CKM matrix then has three angles and one phase. This residual phase breaks the combined symmetry of charge conjugation and parity usually referred to as CP. Besides the empirically small QCD vacuum angle, this phase is the sole breaking of CP within the SM.

There are also interactions mediated by the charged $W$ bosons connecting the two components of the other $\mathrm{SU}(2)_{\mathrm{L}}$ fermion doublets, $L_{L}^{i}$. However, in this case the upper component of these doublets, the neutrinos $\nu_{L}^{i}$, are massless in the SM. This is because there is no Yukawa interaction term with the Higgs that produces a Dirac mass term for the neutrinos after electroweak symmetry breaking. Moreover, Majorana mass terms for the SM neutrinos would explicitly break the $\mathrm{SU}(2)_{\mathrm{L}}$ gauge symmetry and are therefore forbidden. We are then free to apply any rotation matrix on them and we can choose $U_{e}$, the rotation matrix acting on the lower components of $L_{L}^{i}$ fields. This matrix then cancels, leaving these types of interactions diagonal in flavour space. However, it is known from neutrino oscillation experiments that the neutrinos have tiny but non-zero masses [13]. An explanation of this fact necessarily requires new physics. It also implies that these fields have their own rotation matrix. The situation is then similar to the $Q_{L}$ case and results in a matrix analogous to the CKM matrix called the Pontecorvo-Maki-Nakagawa-Sakata (PMNS) matrix. 


\subsection{The Standard Model as an effective field the- ory and the hierarchy problem}

The SM is a renormalizable theory: all divergences coming from any computation can be removed by renormalizing the finite number of coefficients in the SM Lagrangian. However, a more modern point of view is to consider the SM as a low energy effective field theory of a more fundamental Lagrangian $\mathcal{L}^{\mathrm{UV}}$. This Lagrangian, besides including the SM Lagrangian, may have new quantum fields with large masses coupled in some way to the SM field content. At an energy scale below the characteristic scale of new physics, which we denote by $\Lambda$, the appropriate procedure is to integrate out the heavy degrees of freedom. This results in a non-renormalizable Lagrangian including every term in the SM Lagrangian plus every possible higher dimensional operator allowed by the symmetries of the theory. Higher dimensional operators have mass dimension $\geq 5$ or, equivalently, coefficients with mass dimension $\leq-1$. As an example, there is a single dimension five operator called the Weinberg operator [14]

$$
\mathcal{L}^{(5)}=\frac{c^{i j}}{\Lambda}\left(H \cdot L^{i}\right)\left(H \cdot L^{j}\right)
$$

where the $c^{i j}$ are dimensionless numbers, the dots represent $\mathrm{SU}(2)_{\mathrm{L}}$ contractions, and we have used two-spinor notation to denote the $L$ fields. This operator breaks each lepton number and will only be generated if the underlying theory also breaks these symmetries. Additionally, once electroweak symmetry breaking is taken into account, this operator will generate Majorana neutrino masses. Continuing, we write

$$
\mathcal{L}_{\mathrm{EFT}}^{\mathrm{SM}}=\mathcal{L}^{\mathrm{SM}}+\mathcal{L}^{(5)}+\mathcal{L}^{(6)}+\mathcal{L}^{(7)}+\ldots \quad \text { with } \quad \mathcal{L}^{(d)}=\sum_{i} \frac{c_{i}^{(d)}}{\Lambda^{d-4}} \mathcal{O}_{i}^{(d)}
$$


where the sum over $i$ for a given mass dimension $d$ includes every allowed operator with that mass dimension.

A remarkable property of effective field theories is that the only way in which the high energy physics affects predictions made at low energies using the effective field theory is through the determination of the coefficients which make up the low energy effective field theory. In other words, besides determining the low energy coefficients, the exact details of the high energy physics are irrelevant. Except for one important exception, all coefficients in the low energy effective field theory are at most logarithmically sensitive to the scale of new physics $\Lambda$. The one exception is mass squared terms for scalar particles, which are quadratically sensitive to $\Lambda$. There is one such term in the SM, the $\mu^{2}$ term in the scalar potential of the Higgs as given in equation 2.43. This parameter can be thought of as the renormalized value equal to $-\mu^{2}=2 \lambda v^{2}=m_{h}^{2} / 2 \approx(88.4 \mathrm{GeV})^{2}$ where we used that the mass of the recently discovered Higgs boson is $m_{h} \approx 125 \mathrm{GeV}$. The quadratic sensitivity of this parameter to the scale of new physics is due to radiative corrections in the form of loop diagrams. Particularly, the renormalized value is the sum of the bare quantity, $\mu_{0}^{2}$, and the radiative corrections so that we can write schematically $\mu^{2} \sim \mu_{0}^{2}+\Lambda^{2}$. Increasing the value of $\Lambda$ requires increasingly delicate cancellations between the bare quantity and the radiative corrections. The classic example is to consider the case were $\Lambda$ is equal to the Planck scale, $\Lambda \sim m_{P l} \sim 10^{19} \mathrm{GeV}$ in which case $\mu_{0}^{2} \sim-\left(1+10^{-34}\right) \Lambda^{2}$. Thus, the bare quantity and the radiative corrections must be incredibly fine-tuned with respect to each other to reproduce the observed renormalized value. This quadratic sensitivity of the Higgs potential to the scale of new physics is commonly referred to as the hierarchy problem. One possible solution to the hierarchy problem, supersymmetry, is the subject of the next chapter. 


\section{Chapter 3}

\section{Supersymmetry}

\subsection{Introduction}

Supersymmetry (SUSY) is a spacetime symmetry that relates bosons to fermions. Such particles related to each other by supersymmetry are called superpartners. Supersymmetry then imposes requirements onto the superpartners. For example, in a supersymmetric theory, the masses of the superpartners must be equal. Additionally, all possible interaction terms involving the superpartners are connected in some delicate manner. More generally, supersymmetry forces any theory of elementary particles to have an intricate structure, and this structure results in many desirable properties.

Perhaps the most famous property of supersymmetric theories is the cancellation of quadratically divergent contributions to the mass squared terms of scalar particles. Thus, a potential cure to the hierarchy problem, as initially described in section 2.7, is to apply supersymmetry to the SM. A simple argument to see that this is the case is to note that fermion masses are at most logarithmically sensitive to the scale of new physics instead of linearly sensitive. This follows from the presence of 

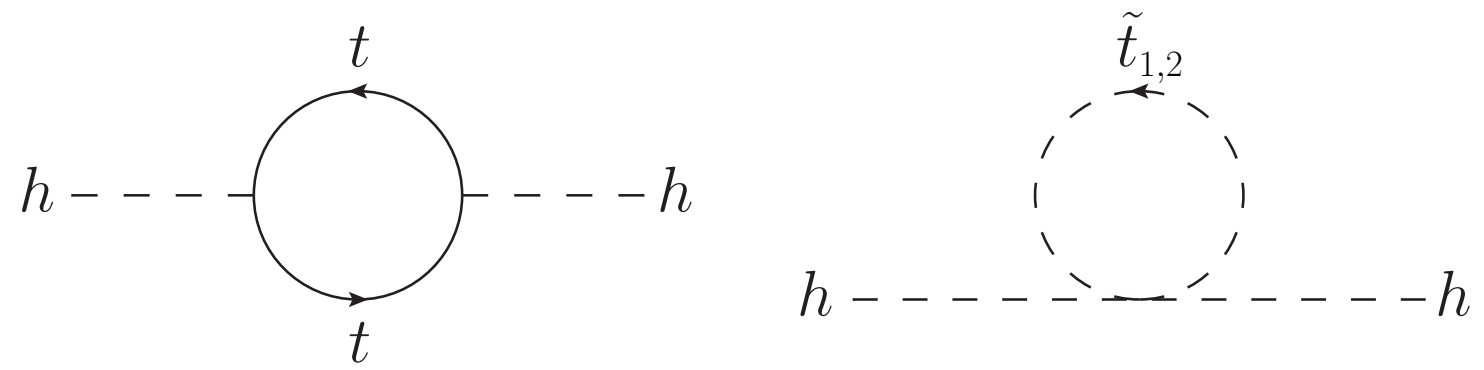

Figure 3.1: Quadratically divergent one loop diagrams with top quarks and squarks contributing to the squared mass of the Higgs. The quadratic divergences cancel between the two diagrams.

a chiral symmetry in the limit that the fermion mass is set to zero. Supersymmetry links scalars to fermions and this transmits the chiral symmetry protection from the fermion mass term onto the scalar mass squared term. Another way to understand this feature is diagrammatically. Quadratic divergences to scalar mass squared terms cancel when summing over all possible Feynman diagrams contributing to the scalar two-point function. As an example, consider the top quark contribution to the squared mass of the SM Higgs at one loop as shown in the left diagram in figure 3.1. In a supersymmetric extension, the Higgs also couples to the superpartners of the top quark, the top squarks $\tilde{t}_{1,2}$, and there are contributions to the Higgs squared mass at one loop from these particles too, as shown in the right diagram of figure 3.1. Both diagrams are quadratically divergent, but the quadratic divergences cancel when the diagrams are summed. This feature extends to all types of particles and to all loop levels, and even if supersymmetry is broken provided the breaking is soft.

Besides potentially alleviating the hierarchy problem, supersymmetry has many other benefits, two of which we discuss here. The first is if the discrete $\mathbb{Z}_{2}$ symmetry known as $R$-parity (discussed in greater detail in section 3.7.2) is unbroken, then the lightest supersymmetric particle is necessarily stable. It may then be a viable 
dark matter candidate. Indeed, supersymmetric extensions of the SM can result in many different types of dark matter candidates depending on the exact details of the model. The other benefit is that supersymmetric extensions of the SM help facilitate the unification of the gauge couplings. In other words, if the three gauge couplings are evolved up to high energy scales using their renormalization group equations, then they are seen to converge to a single value at an energy scale of approximately $10^{16} \mathrm{GeV}$ in many supersymmetric theories. This is beneficial if one wants to embed the SM gauge group into a larger group such as $\mathrm{SU}(5)$ or $\mathrm{SO}(10)$ as in most Grand Unified Theories (GUTs). In contrast, if the three gauge couplings are evolved to high energy scales within the SM, then they do not converge to a single value.

This chapter will attempt to give a brief introduction to supersymmetry. It begins with a discussion of the supersymmetry algebra. Different types of superfields are then considered. The procedure to construct supersymmetric Lagrangians is then outlined. The forms of general soft supersymmetry breaking terms are then presented. The scalar potential is then determined. The results from these sections are then applied to the SM resulting in the Minimal Supersymmetric Standard Model (MSSM). Possible mediation mechanisms which communicate the breaking of supersymmetry to the MSSM are then briefly reviewed. Finally, this chapter concludes with a general treatment of $R$-symmetries. This chapter draws inspiration from the textbook by Labelle [15] and the review by Martin [16].

\subsection{The supersymmetry algebra}

As discussed in section 2.2, the symmetries of spacetime are determined by the Poincaré algebra. There are four generators of time and space translations $P^{\mu}$ and six Lorentz generators $\mathcal{J}^{\mu \nu}$, three of which can be identified with rotations $J_{i}=\frac{1}{2} \epsilon_{i j k} \mathcal{J}^{j k}$ 
and the other three with boosts $K_{i}=\mathcal{J}^{0 i}$. The Poincaré algebra in terms of $\mathcal{J}^{\mu \nu}$ and $P^{\mu}$ is given by equations 2.3 and 2.6 . We noted in section 2.3 that elementary particles transform under representations of the Poincaré group. Elementary particles can also transform under internal symmetry groups, for example, gauge symmetries. Let us denote the generators of an internal symmetry group by $T^{a}$ and their algebra by $\left[T^{a}, T^{b}\right]=i f^{a b c} T^{c}$. An important result proved by Coleman and Mandula [17] is that, under a few physically reasonable conditions, the generators of internal symmetries $T^{a}$ and the Poincaré generators $P^{\mu}$ and $\mathcal{J}^{\mu \nu}$ cannot have non-trivial commutation relations, i.e. they necessarily commute. In other words, internal symmetries and spacetime symmetries do not mix. However, there is one way to circumvent this no-go theorem. If the new symmetry generators are fermionic so that they satisfy anticommutation relations, then they can potentially have non-trivial commutation relations with the Poincaré generators. Supersymmetry makes use of this loophole.

The symmetry generators of supersymmetry are fermionic: they transform as left-handed or right-handed spinors under the Lorentz group. They are denoted $Q_{\alpha}$ and its hermitian conjugate $Q_{\dot{\alpha}}^{\dagger}$. Here we have again made use of the van der Waerden undotted and dotted spinor index notation introduced in section 2.3. These symmetry generators, $Q_{\alpha}$ and $Q_{\dot{\alpha}}^{\dagger}$, generate what is known as $N=1$ supersymmetry, with $N$ representing the number of fermionic symmetry generator pairs introduced. According to the Haag-Lopuszanski-Sohnius theorem [18], every possible non-trivial extension of the Poincaré algebra is a type of supersymmetry with integer $N$ fermionic symmetry generator pairs. The $N>1$ supersymmetries are collectively referred to as extended supersymmetry. It turns out that the only phenomenologically interesting supersymmetry, at least at modern collider centre of mass energies, is $N=1$ supersymmetry. This is because fermions in larger $N$ supersymmetries are non-chiral, in contrast to 
the chiral fermions in the SM. Additionally, the largest extended supersymmetry is generally considered to be $N=8$. Larger values of $N$ lead to massless particles with spins $>2$ and it is known that such particles cannot interact in a quantum field theory. Extended supersymmetry will no longer be discussed in this thesis.

The $N=1$ supersymmetry algebra is given by

$$
\begin{aligned}
{\left[P^{\mu}, P^{\nu}\right] } & =0, \quad\left[\mathcal{J}^{\mu \nu}, P^{\rho}\right]=i\left(g^{\nu \rho} P^{\mu}-g^{\mu \rho} P^{\nu}\right) \\
{\left[\mathcal{J}^{\mu \nu}, \mathcal{J}^{\rho \sigma}\right] } & =i\left(g^{\nu \rho} \mathcal{J}^{\mu \sigma}-g^{\mu \rho} \mathcal{J}^{\nu \sigma}-g^{\nu \sigma} \mathcal{J}^{\mu \rho}+g^{\mu \sigma} \mathcal{J}^{\nu \rho}\right), \\
\left\{Q_{\alpha}, Q_{\beta}\right\} & =0, \quad\left\{Q_{\dot{\alpha}}^{\dagger}, Q_{\dot{\beta}}^{\dagger}\right\}=0, \quad\left\{Q_{\alpha}, Q_{\dot{\beta}}^{\dagger}\right\}=\sigma_{\alpha \dot{\beta}}^{\mu} P_{\mu} \\
{\left[Q_{\alpha}, P^{\mu}\right] } & =0, \quad\left[Q_{\dot{\alpha}}^{\dagger}, P^{\mu}\right]=0, \\
{\left[Q_{\alpha}, \mathcal{J}^{\mu \nu}\right] } & =\left(\sigma^{\mu \nu}\right)_{\alpha}^{\beta} Q_{\beta}, \quad\left[Q_{\dot{\alpha}}^{\dagger}, \mathcal{J}^{\mu \nu}\right]=-Q_{\dot{\beta}}^{\dagger}\left(\bar{\sigma}^{\mu \nu}\right)_{\dot{\alpha}}^{\dot{\beta}}
\end{aligned}
$$

where we have rewritten the Poincaré algebra for completeness. The matrices $\sigma^{\mu \nu}$ and $\bar{\sigma}^{\mu \nu}$ are $\left(\sigma^{\mu \nu}\right)_{\alpha}{ }^{\beta}=\frac{i}{4}\left(\sigma^{\mu} \bar{\sigma}^{\nu}-\sigma^{\nu} \bar{\sigma}^{\mu}\right)_{\alpha}{ }^{\beta}$ and $\left(\bar{\sigma}^{\mu \nu}\right)_{\dot{\beta}}^{\dot{\alpha}}=\frac{i}{4}\left(\bar{\sigma}^{\mu} \sigma^{\nu}-\bar{\sigma}^{\nu} \sigma^{\mu}\right)_{\dot{\beta}}^{\dot{\alpha}}$ where the $\sigma_{\alpha \dot{\beta}}^{\mu}$ and $\bar{\sigma}^{\mu \dot{\alpha} \beta}$ matrices are the same as those introduced in section 2.3. Notice that the anticommutator $\left\{Q_{\alpha}, Q_{\dot{\beta}}^{\dagger}\right\}=\sigma_{\alpha \dot{\beta}}^{\mu} P_{\mu}$ shows that the supersymmetry generators $Q_{\alpha}$ and $Q_{\dot{\alpha}}^{\dagger}$ have dimensions of $[\text { Energy }]^{1 / 2}$.

In a supersymmetric theory, elementary particle states transform in irreducible representations of the supersymmetry algebra, called supermultiplets. The effect of the supercharges $Q$ and $Q^{\dagger}$ is to interchange bosonic and fermionic states within the supermultiplet. Furthermore, each supermultiplet must contain the same number of bosonic and fermionic degrees of freedom. Since we are considering $N=1$ supersymmetry, each supermultiplet contains only one on-shell bosonic and fermionic state. The simplest type is called a chiral supermultiplet and consists of a complex scalar field and a single Weyl spinor. The next type is called a vector supermultiplet and 
consists of a single Weyl spinor and a vector boson. Finally, if we consider gravity, then there is a supermultiplet consisting of the spin 2 graviton and the spin $3 / 2$ gravitino. The generators of supersymmetry commute with the generators of gauge transformations and this implies that each component of these types of supermultiplets necessarily have the same gauge charges. The generators of supersymmetry also commute with the mass operator $P_{\mu} P^{\mu}$ and this implies that each component of these types of supermultiplets necessarily have the same mass, provided supersymmetry is unbroken.

\subsection{Superfields}

This thesis will make use of the superfield formalism to describe these supermultiplets. To do so, the first step is to extend spacetime to superspace which has the four spacetime coordinates $x^{\mu}$, but also four fermionic (Grassmann) coordinates arranged

into a left-handed spinor $\theta_{\alpha}$ and its hermitian conjugate $\theta_{\dot{\alpha}}^{\dagger}$. Superfields are then functions of $x^{\mu}, \theta$, and $\theta^{\dagger}$.

Consider performing a superspace transformation parameterized by $\zeta$. We will consider the parameter $\zeta$ to be independent of spacetime. Thus, we are considering global supersymmetry. Making $\zeta$ spacetime dependent results in local supersymmetry and forces one to consider gravity. These types of theories are referred to as supergravity. Carrying on with the global supersymmetry transformation, a general scalar superfield $\mathcal{S}\left(x, \theta, \theta^{\dagger}\right)$ transforms

$$
\begin{aligned}
\mathcal{S}\left(x, \theta, \theta^{\dagger}\right) & \rightarrow U\left(\zeta, \zeta^{\dagger}\right)^{-1} \mathcal{S}\left(x, \theta, \theta^{\dagger}\right) U\left(\zeta, \zeta^{\dagger}\right) \\
& =\mathcal{S}\left(x-a, \theta-\zeta, \theta^{\dagger}-\zeta^{\dagger}\right)=e^{i\left(\zeta \hat{Q}+\zeta^{\dagger} \hat{Q}^{\dagger}\right)} \mathcal{S}\left(x, \theta, \theta^{\dagger}\right)
\end{aligned}
$$


where $a^{\mu}=\frac{i}{2} \zeta \sigma^{\mu} \theta^{\dagger}-\frac{i}{2} \theta \sigma^{\mu} \zeta^{\dagger}$ (cf. equations 2.7 and 2.9). Since the argument of an exponential is dimensionless, we find that $\theta$ and $\theta^{\dagger}$ have dimensions of $[\text { Energy }]^{-1 / 2}$. Taylor series expanding both sides of equation 3.2 then results in a representation of the supersymmetry charges

$$
\begin{aligned}
\hat{Q}_{\alpha} & =i \partial_{\alpha}-\frac{1}{2} \sigma_{\alpha \dot{\beta}}^{\mu} \theta^{\dagger \dot{\beta}} \partial_{\mu} & \hat{Q}^{\alpha} & =-i \partial^{\alpha}+\frac{1}{2} \theta_{\dot{\beta}}^{\dagger} \bar{\sigma}^{\mu \dot{\beta} \alpha} \partial_{\mu} \\
\hat{Q}_{\dot{\alpha}}^{\dagger} & =-i \partial_{\dot{\alpha}}+\frac{1}{2} \theta^{\beta} \sigma_{\beta \dot{\alpha}}^{\mu} \partial_{\mu} & \hat{Q}^{\dagger \dot{\alpha}} & =i \partial^{\dot{\alpha}}-\frac{1}{2} \bar{\sigma}^{\mu \dot{\alpha} \beta} \theta_{\beta} \partial_{\mu} .
\end{aligned}
$$

In these expressions, $\partial_{\alpha} \equiv \frac{\partial}{\partial \theta^{\alpha}}, \partial^{\alpha} \equiv \frac{\partial}{\partial \theta_{\alpha}}$, and the dotted index derivatives are defined similarly with $\theta \rightarrow \theta^{\dagger}$. These representations of $Q$ and $Q^{\dagger}$ can be compared with the representation of $P^{\mu}$ and $\mathcal{J}^{\mu \nu}$ acting on a scalar field $\hat{P}^{\mu}=i \partial^{\mu}$ and $\hat{\mathcal{J}}^{\mu \nu}=$ $i\left(x^{\mu} \partial^{\nu}-x^{\nu} \partial^{\mu}\right)$ as given by equations 2.8 and 2.10 .

Due to their Grassmann nature, an expansion of a superfield in terms of $\theta$ and $\theta^{\dagger}$ quickly terminates after a finite number of terms. This leads to the most general scalar superfield

$$
\begin{aligned}
\mathcal{S}\left(x, \theta, \theta^{\dagger}\right)= & A(x)+\theta \chi(x)+\theta^{\dagger} \psi^{\dagger}(x)+\theta \theta F(x)+\theta^{\dagger} \theta^{\dagger} G(x)+\theta \sigma^{\mu} \theta^{\dagger} V_{\mu}(x) \\
& +\theta \theta \theta^{\dagger} \lambda^{\dagger}(x)+\theta^{\dagger} \theta^{\dagger} \theta \xi(x)+\theta \theta \theta^{\dagger} \theta^{\dagger} D(x)
\end{aligned}
$$

where $A(x), F(x), G(x)$, and $D(x)$ are complex scalar fields, $\chi(x), \psi(x), \lambda(x)$, and $\xi(x)$ are left-handed spinor fields, and $V_{\mu}(x)$ is a complex vector field. Such a superfield has too many degrees of freedom to be identified with a chiral or vector supermultiplet. Indeed, the most general scalar superfield is reducible with respect to supersymmetry. It is possible to determine the superfields for the chiral and vector supermultiplets by applying supersymmetrically invariant constraints onto the most general superfield. 


\subsubsection{Chiral superfields}

The standard procedure to construct chiral (or left-chiral) superfields is to apply a derivative operator $\bar{D}_{\dot{\alpha}}$ onto the most general superfield and enforce the constraint $\bar{D}_{\dot{\alpha}} \Phi=0$. Similarly, antichiral (or right-chiral) superfields can be constructed by applying another derivative operator $D_{\alpha}$ onto the most general superfield and enforcing the constraint $D_{\alpha} \Phi=0$. These derivative operators are

$$
D_{\alpha}=\partial_{\alpha}-\frac{i}{2} \sigma_{\alpha \dot{\beta}}^{\mu} \theta^{\dagger \dot{\beta}} \partial_{\mu} \quad \text { and } \quad \bar{D}_{\dot{\alpha}}=\partial_{\dot{\alpha}}-\frac{i}{2} \theta^{\beta} \sigma_{\beta \dot{\alpha}}^{\mu} \partial_{\mu} .
$$

Crucially, both derivative operators satisfy

$$
\left\{\hat{Q}_{\alpha}, D_{\beta}\right\}=\left\{\hat{Q}_{\dot{\alpha}}^{\dagger}, D_{\beta}\right\}=\left\{\hat{Q}_{\alpha}, \bar{D}_{\dot{\beta}}\right\}=\left\{\hat{Q}_{\dot{\alpha}}^{\dagger}, \bar{D}_{\dot{\beta}}\right\}=0
$$

which is necessary for the constraint equations $D_{\alpha} \Phi=0$ and $\bar{D}_{\dot{\alpha}} \Phi=0$ to be preserved under a supersymmetry transformation. The indices on these operators can also be raised and this gives

$$
D^{\alpha}=-\partial^{\alpha}+\frac{i}{2} \theta_{\dot{\beta}}^{\dagger} \bar{\sigma}^{\mu \dot{\beta} \alpha} \partial_{\mu} \quad \text { and } \quad \bar{D}^{\dot{\alpha}}=-\partial^{\dot{\alpha}}+\frac{i}{2} \bar{\sigma}^{\mu \dot{\alpha} \beta} \theta_{\beta} \partial_{\mu} .
$$

To determine the superfield satisfying $\bar{D}_{\dot{\alpha}} \Phi=0$, it is useful to introduce the coordinate

$$
y^{\mu}=x^{\mu}-\frac{i}{2} \theta \sigma^{\mu} \theta^{\dagger}
$$

which satisfies $\bar{D}_{\dot{\alpha}} y^{\mu}=0$. A superfield which depends only on $y^{\mu}$ and $\theta$ is then

guaranteed to satisfy $\bar{D}_{\dot{\alpha}} \Phi(y, \theta)=0$. Expanding $\Phi(y, \theta)$ in terms of its Grassmann 
variables then results in

$$
\Phi(y, \theta)=\phi(y)+\theta \chi(y)+\frac{1}{2} \theta \theta F(y)
$$

which can be further expanded to yield

$$
\begin{aligned}
\Phi\left(x, \theta, \theta^{\dagger}\right)= & \phi(x)-\frac{i}{2} \theta \sigma^{\mu} \theta^{\dagger} \partial_{\mu} \phi(x)-\frac{1}{16} \theta \theta \theta^{\dagger} \theta^{\dagger} \square \phi(x) \\
& +\theta \chi(x)-\frac{i}{2} \theta \sigma^{\mu} \theta^{\dagger} \theta \partial_{\mu} \chi(x)+\frac{1}{2} \theta \theta F(x)
\end{aligned}
$$

where $\square=\partial_{\mu} \partial^{\mu}$ is the d'Alembertian operator. The field content of a chiral superfield is then a complex scalar $\phi$, a left-handed spinor $\chi$, and another complex scalar field $F$. As will be seen, the field $F$ does not propagate and can be solved for through its equation of motion in terms of other scalar fields. These types of fields are known as auxiliary fields. The dimension of a chiral superfield $\Phi$ is the same as the dimension of its complex scalar field $\phi$, that is, [Energy $]^{1}$.

Under a supersymmetry transformation, the component fields of a chiral superfield transform as follows

$$
\begin{aligned}
& \phi \rightarrow \phi+\zeta \chi \\
& \chi \rightarrow \chi-i \sigma^{\mu} \zeta^{\dagger} \partial_{\mu} \phi+\zeta F \\
& F \rightarrow F-i \zeta^{\dagger} \bar{\sigma}^{\mu} \partial_{\mu} \chi .
\end{aligned}
$$

Notice that the $F$ term transforms as a total derivative under supersymmetry. This can be seen from the requirement that the fields transform linearly and from dimensional analysis. 
If we take the hermitian conjugate of a chiral superfield, then

$$
\Phi^{\dagger}\left(y^{\mu}, \theta\right)=\Phi\left(y^{\mu \dagger}, \theta^{\dagger}\right)=\Phi\left(z^{\mu}, \theta^{\dagger}\right)
$$

where $z^{\mu}=x^{\mu}+\frac{i}{2} \theta \sigma^{\mu} \theta^{\dagger}$. The coordinate $z^{\mu}$ satisfies $D_{\alpha} z^{\mu}=0$ and this implies $D_{\alpha} \Phi\left(z^{\mu}, \theta^{\dagger}\right)=0$ so that $\Phi\left(z^{\mu}, \theta^{\dagger}\right)$ is an antichiral superfield. Thus, the hermitian conjugate of a chiral superfield is antichiral and the hermitian conjugate of an antichiral superfield is chiral.

It is beneficial to note that the sum or product of two chiral superfields is also a chiral superfield. This follows because the derivative operator $\bar{D}_{\dot{\alpha}}$ is both a linear operator and satisfies the product rule. Thus, a holomorphic function of chiral superfields is also a chiral superfield. Similarly, the derivative operator $D_{\alpha}$ is also both a linear operator and satisfies the product rule. This implies that a holomorphic function of antichiral superfields is also antichiral.

\subsubsection{Vector superfields}

The bosonic component of a vector superfield is a massless gauge field $A_{\mu}(x)$. Consider initially a gauge field based on a local U(1) symmetry. We have seen that such a gauge field transforms under a gauge transformation, given by equation 2.15. Similarly, vector superfields transform under a supergauge transformation, which will be shown momentarily. To construct a vector superfield $\mathcal{V}$ for this gauge symmetry, we first start with a general superfield as given in equation 3.4 and require it to be real, $\mathcal{V}^{\dagger}=\mathcal{V}$. Importantly, this condition is consistent with supersymmetry. A vector superfield can then be represented as follows

$$
\mathcal{V}=C(x)+\frac{i}{\sqrt{2}} \theta \rho(x)-\frac{i}{\sqrt{2}} \theta^{\dagger} \rho^{\dagger}(x)
$$




$$
\begin{aligned}
& +\frac{i}{4} \theta \theta(M(x)+i N(x))-\frac{i}{4} \theta^{\dagger} \theta^{\dagger}(M(x)-i N(x))+\frac{1}{2} \theta \sigma^{\mu} \theta^{\dagger} A_{\mu}(x) \\
& +\frac{1}{2 \sqrt{2}} \theta \theta\left(\theta^{\dagger} \lambda^{\dagger}(x)+\frac{1}{2} \theta^{\dagger} \bar{\sigma}^{\mu} \partial_{\mu} \rho(x)\right)+\frac{1}{2 \sqrt{2}} \theta^{\dagger} \theta^{\dagger}\left(\theta \lambda(x)-\frac{1}{2} \theta \sigma^{\mu} \partial_{\mu} \rho^{\dagger}(x)\right) \\
& -\frac{1}{8} \theta \theta \theta^{\dagger} \theta^{\dagger}\left(D(x)+\frac{1}{2} \square C(x)\right)
\end{aligned}
$$

where $C, M, N$, and $D$ are real scalar fields, $A_{\mu}$ is a real vector field, and $\rho$ and $\lambda$ are left-handed spinors. Vector fields are dimensionless as can be seen by examining the $\frac{1}{2} \theta \sigma^{\mu} \theta^{\dagger} A_{\mu}(x)$ term.

To perform a supergauge transformation, the first step is to promote the gauge parameter $\alpha(x)$ to a chiral superfield $\Lambda\left(x, \theta, \theta^{\dagger}\right)$. The form of $\Lambda$ is then the same as in equation 3.10, except $\Lambda$ is now dimensionless. Under a supergauge transformation, $\mathcal{V}$ then transforms

$$
\begin{aligned}
\mathcal{V} \rightarrow \mathcal{V}+i\left(\Lambda-\Lambda^{\dagger}\right)=\mathcal{V} & +i\left(\phi_{\Lambda}-\phi_{\Lambda}^{\dagger}\right)+i\left(\theta \chi_{\Lambda}-\theta^{\dagger} \chi_{\Lambda}^{\dagger}\right)+\frac{i}{2} \theta \theta F_{\Lambda}-\frac{i}{2} \theta^{\dagger} \theta^{\dagger} F_{\Lambda}^{\dagger} \\
& +\frac{1}{2} \theta \sigma^{\mu} \theta^{\dagger} \partial_{\mu}\left(\phi_{\Lambda}+\phi_{\Lambda}^{\dagger}\right)+\frac{1}{4}\left(\theta \theta \theta^{\dagger} \bar{\sigma}^{\mu} \partial_{\mu} \chi_{\Lambda}-\theta^{\dagger} \theta^{\dagger} \theta \sigma^{\mu} \partial_{\mu} \chi_{\Lambda}^{\dagger}\right) \\
& -\frac{i}{16} \theta \theta \theta^{\dagger} \theta^{\dagger} \square\left(\phi_{\Lambda}-\phi_{\Lambda}^{\dagger}\right) .
\end{aligned}
$$

Notice that $i\left(\Lambda-\Lambda^{\dagger}\right)$ is real as it must be since $\mathcal{V}$ is real. Equations 3.13 and 3.14 then imply that the individual component fields of the vector superfield transform

$$
\begin{array}{ccc}
C \rightarrow C+i\left(\phi_{\Lambda}-\phi_{\Lambda}^{\dagger}\right) & M+i N \rightarrow M+i N+2 F_{\Lambda} & \rho \rightarrow \rho+\sqrt{2} \chi_{\Lambda} \\
A_{\mu} \rightarrow A_{\mu}+\partial_{\mu}\left(\phi_{\Lambda}+\phi_{\Lambda}^{\dagger}\right) & D \rightarrow D & \lambda \rightarrow \lambda .
\end{array}
$$

As shown by these transformations, the fields $D$ and $\lambda$ for an abelian vector superfield are both gauge singlets. Moreover, this gauge freedom can be used to set $C, M, N$, 
and $\rho$ to zero. The vector superfield then becomes

$$
\mathcal{V}=\frac{1}{2} \theta \sigma^{\mu} \theta^{\dagger} A_{\mu}+\frac{1}{2 \sqrt{2}} \theta \theta \theta^{\dagger} \lambda^{\dagger}+\frac{1}{2 \sqrt{2}} \theta^{\dagger} \theta^{\dagger} \theta \lambda-\frac{1}{8} \theta \theta \theta^{\dagger} \theta^{\dagger} D
$$

This gauge choice is known as Wess-Zumino gauge. The fermion $\lambda$ is called a gaugino and the real scalar field $D$ is another example of an auxiliary field that can be solved for through its equation of motion in terms of other scalar fields. The Wess-Zumino gauge also does not completely fix the gauge either. There is still a residual gauge freedom where

$$
A_{\mu} \rightarrow A_{\mu}+\partial_{\mu}\left(2 \operatorname{Re}\left[\phi_{\Lambda}\right]\right)
$$

This is simply the standard gauge freedom initially considered in equation 2.15 with a slightly different scaling. ${ }^{1}$

Under a supersymmetry transformation, the component fields $A_{\mu}, \lambda$, and $D$ transform as follows ${ }^{2}$

$$
\begin{gathered}
A^{\mu} \rightarrow A^{\mu}-\frac{1}{\sqrt{2}}\left(\zeta^{\dagger} \bar{\sigma}^{\mu} \lambda+\lambda^{\dagger} \bar{\sigma}^{\mu} \zeta\right) \\
\lambda \rightarrow \lambda-\frac{i}{2 \sqrt{2}} F_{\mu \nu} \sigma^{\mu} \bar{\sigma}^{\nu} \zeta-\frac{1}{\sqrt{2}} D \zeta \\
D \rightarrow D+\frac{i}{\sqrt{2}} \zeta^{\dagger} \bar{\sigma}^{\mu} \partial_{\mu} \lambda-\frac{i}{\sqrt{2}} \partial_{\mu} \lambda^{\dagger} \bar{\sigma}^{\mu} \zeta .
\end{gathered}
$$

Like the $F$ term in a chiral superfield, the $D$ term for an abelian vector superfield

\footnotetext{
${ }^{1}$ Gauge transformations in this chapter will have a slightly different scaling compared to those in chapter 2. For an abelian gauge symmetry, the vector field will now transform $A_{\mu} \rightarrow A_{\mu}+\partial_{\mu} \alpha$ and a scalar or fermionic field $\varphi$ will now transform $\varphi \rightarrow e^{i q e \alpha} \varphi$. For a non-abelian gauge symmetry, the corresponding transformations will now be $A_{\mu}^{a} \rightarrow A_{\mu}^{a}+\partial_{\mu} \alpha^{a}-g f^{a b c} \alpha^{b} A_{\mu}^{c}$ and $\varphi \rightarrow e^{i g \alpha^{a} t_{r}^{a}} \varphi$.

${ }^{2}$ In these transformations, the parameter $\zeta$ has been scaled by a factor of $-\frac{1}{\sqrt{2}}$. This has been done so that these transformations will not need to be modified once the vector superfield is coupled to chiral superfields.
} 
transforms as a total derivative under supersymmetry. This again can be seen from the requirement that the fields transform linearly and from dimensional analysis.

These results naturally generalize to the case of a gauge symmetry based on a non-abelian symmetry group. If the non-abelian symmetry group has $n$ generators, then there are $n$ vector superfields $\mathcal{V}^{a}$. In Wess-Zumino gauge each take the form of equation 3.16 where now the three component fields have an $a$ index as well. There is still a residual gauge freedom similar to equation 3.17 but now the gauge fields $A_{\mu}^{a}$ transform like equation 2.34. The $D^{a}$ and $\lambda^{a}$ fields are also no longer gauge invariant and now transform in the adjoint representation of the symmetry group, similar to equation 2.36 where the generators of the adjoint representation are $\left[T_{\text {adj }}^{a}\right]_{b c}=-i f^{a b c}$. Finally, the three fields transform as in equation 3.18 under a supersymmetry transformation except with the following two modifications: each field requires an $a$ index including $F_{\mu \nu}^{a}$ whose form is given by equation 2.33 and the derivatives acting on $\lambda^{a}$ in the transformation of $D^{a}$ are both promoted to covariant derivatives.

\subsection{Supersymmetrically invariant Lagrangians}

To construct supersymmetrically invariant Lagrangians, we use the fact that the $F$ term of a chiral superfield and the $D$ term of a vector superfield both transform under supersymmetry as total derivatives. Here we are assuming that the $F$ term and the $D$ term of these types of superfields are both gauge singlets. Also, by $F$ term and $D$ term we formally mean the field, or combination of fields, multiplying the $\theta \theta / 2$ and the $\theta \theta \theta^{\dagger} \theta^{\dagger} / 4$ Grassmann terms, respectively. In fact, the $D$ term of any superfield necessarily transforms like a total derivative under supersymmetry if it is a gauge singlet. This again follows for the same reasons as outlined in the chiral and vector 
superfield cases.

To isolate these terms from a general superfield, we can integrate over the Grassmann coordinates. The relevant integrals we need $\operatorname{are}^{3}$

$$
\frac{1}{2} \int d^{2} \theta \theta \theta=1 \quad \text { and } \quad \frac{1}{4} \int d^{2} \theta d^{2} \theta^{\dagger} \theta \theta \theta^{\dagger} \theta^{\dagger}=1
$$

from which it follows

$$
\left.\int d^{2} \theta \mathcal{S}\left(x, \theta, \theta^{\dagger}\right)\right|_{\theta^{\dagger}=0}=\left.\mathcal{S}\left(x, \theta, \theta^{\dagger}\right)\right|_{\theta \theta / 2}=\left.\mathcal{S}\left(x, \theta, \theta^{\dagger}\right)\right|_{F}
$$

and

$$
\int d^{2} \theta d^{2} \theta^{\dagger} \mathcal{S}\left(x, \theta, \theta^{\dagger}\right)=\left.\mathcal{S}\left(x, \theta, \theta^{\dagger}\right)\right|_{\theta \theta \theta^{\dagger} \theta^{\dagger} / 4}=\left.\mathcal{S}\left(x, \theta, \theta^{\dagger}\right)\right|_{D}
$$

where $\mathcal{S}\left(x, \theta, \theta^{\dagger}\right)$ represents any type of superfield. From the integrals in equation 3.19, we see that the dimensions of $d^{2} \theta$ and $d^{2} \theta d^{2} \theta^{\dagger}$ are $[\text { Energy }]^{1}$ and $[\text { Energy }]^{2}$, respectively. Thus, if $\mathcal{S}\left(x, \theta, \theta^{\dagger}\right)$ has dimension [Energy $]^{d}$, then the $F$ term has dimension $[\text { Energy }]^{d+1}$ and the $D$ term has dimension $[\text { Energy }]^{d+2}$. We will only consider renormalizable supersymmetric Lagrangians so that the superfield will have $d \leq 2$ if we project out the $D$ term and $d \leq 3$ if we project out the $F$ term.

\subsubsection{Kinetic terms for chiral superfields}

Consider first a gauge singlet chiral superfield $\Phi$ as given in equation 3.10. If we multiply this superfield by its hermitian conjugate (which is an antichiral superfield) we then obtain another (real) superfield. The kinetic terms for $\phi, \chi$, and $F$ are given

\footnotetext{
${ }^{3}$ Another notation for $d^{2} \theta d^{2} \theta^{\dagger}$ is $d^{4} \theta$.
} 
by projecting out the $D$ term of the resulting superfield

$$
\mathcal{L}=\left.\Phi^{\dagger} \Phi\right|_{D}=\left(\partial^{\mu} \phi\right)^{\dagger}\left(\partial_{\mu} \phi\right)+i \chi^{\dagger} \bar{\sigma}^{\mu} \partial_{\mu} \chi+F^{\dagger} F
$$

We see that the kinetic term for the $F$ field has no derivatives and therefore does not propagate. This confirms that it is an auxiliary field.

Next, consider a chiral superfield $\Phi$ charged under an abelian gauge symmetry with charge $q$. The vector superfield for the abelian gauge symmetry $\mathcal{V}$ transforms under a supergauge transformation as given in equation 3.14. We take the chiral superfield to transform under the supergauge transformation as

$$
\Phi \rightarrow e^{2 i q e \Lambda} \Phi
$$

where $e$ is the coupling constant. The product $\Phi^{\dagger} e^{-2 q e \mathcal{V}} \Phi$ is then supergauge invariant and projecting out its $D$ term gives

$$
\begin{aligned}
\mathcal{L} & =\left.\Phi^{\dagger} e^{-2 q e \mathcal{V}} \Phi\right|_{D} \\
& =\left(D^{\mu} \phi\right)^{\dagger}\left(D_{\mu} \phi\right)+i \chi^{\dagger} \bar{\sigma}^{\mu} D_{\mu} \chi+F^{\dagger} F+\sqrt{2} q e\left(\phi^{\dagger} \chi \lambda+\phi \chi^{\dagger} \lambda^{\dagger}\right)+q e \phi^{\dagger} \phi D
\end{aligned}
$$

where $D_{\mu}=\partial_{\mu}-i q e A_{\mu}$ for both $\phi$ and $\chi$. We see that this contains the standard kinetic terms with covariant derivatives, but also a new gauge-Yukawa term and an interaction term between the $\phi$ and $D$ fields.

This can be generalized to the case of a non-abelian gauge symmetry. If $\Phi$ transforms in a representation of the gauge symmetry generated by generators $\tau^{a}$, then under a supergauge transformation it transforms $\Phi \rightarrow e^{2 i g \Lambda} \Phi$ where $\Lambda \equiv \Lambda^{a} \tau^{a}$. The generalization of $\Phi^{\dagger} e^{-2 q e \mathcal{V}} \Phi$ is $\Phi^{\dagger} e^{-2 g \mathcal{V}} \Phi$ where now $\mathcal{V} \equiv \mathcal{V}^{a} \tau^{a}$. This implies 
that under a supergauge transformation $e^{-2 g \mathcal{V}} \rightarrow e^{2 i g \Lambda^{\dagger}} e^{-2 g \mathcal{V}} e^{-2 i g \Lambda}$ which is the nonabelian equivalent to equation 3.14. Projecting out the $D$ term then gives the kinetic term for the chiral superfield

$$
\begin{aligned}
\mathcal{L} & =\left.\Phi^{\dagger} e^{-2 g \mathcal{V}} \Phi\right|_{D} \\
& =\left(D^{\mu} \phi\right)^{\dagger}\left(D_{\mu} \phi\right)+i \chi^{\dagger} \bar{\sigma}^{\mu} D_{\mu} \chi+F^{\dagger} F+\sqrt{2} g\left(\phi^{\dagger} \tau^{a} \chi \lambda^{a}+\chi^{\dagger} \tau^{a} \phi \lambda^{a \dagger}\right)+g \phi^{\dagger} \tau^{a} \phi D^{a}
\end{aligned}
$$

where $D_{\mu}=\partial_{\mu}-i g A_{\mu}^{a} \tau^{a}$ for both $\phi$ and $\chi$.

Though not crucial, we note for completeness that the transformations of a chiral superfield under supersymmetry given in equation 3.11 are modified if the chiral superfield is coupled to a vector superfield. In the non-abelian case, the transformations become

$$
\begin{aligned}
& \phi^{i} \rightarrow \phi^{i}+\zeta \chi^{i} \\
& \chi^{i} \rightarrow \chi^{i}-i \sigma^{\mu} \zeta^{\dagger} D_{\mu} \phi^{i}+\zeta F^{i} \\
& F^{i} \rightarrow F^{i}-i \zeta^{\dagger} \bar{\sigma}^{\mu} D_{\mu} \chi^{i}-\sqrt{2} g\left(\tau^{a} \phi\right)^{i} \lambda^{a \dagger} \zeta^{\dagger}
\end{aligned}
$$

and the abelian case is the same except without the $i$ and $a$ indices, $\left(\tau^{a} \phi\right)^{i} \rightarrow \phi$, and $g \rightarrow q e$.

\subsubsection{Kinetic terms for vector superfields}

We would like to write the kinetic terms for the component fields of a vector superfield in the superfield formalism. Consider the case of an abelian gauge symmetry with 
vector superfield $\mathcal{V}$. The first step is to introduce the field-strength superfield

$$
\mathcal{F}_{\alpha}=\bar{D} \bar{D} D_{\alpha} \mathcal{V}
$$

where the derivative operators are those in equations 3.5 and 3.7. The field-strength superfield has dimension of $[\text { Energy }]^{3 / 2}$. Additionally, it can be shown that $\bar{D}_{\dot{\beta}} \mathcal{W}_{\alpha}=0$ and therefore $\mathcal{F}_{\alpha}$ is a chiral superfield. The field-strength superfield is also gauge invariant. The right-hand side of equation 3.27 can be evaluated in Wess-Zumino gauge and results in

$$
\mathcal{F}_{\alpha}(y)=\sqrt{2} \lambda_{\alpha}(y)-D(y) \theta_{\alpha}-F_{\mu \nu}(y)\left(\sigma^{\mu \nu}\right)_{\alpha}^{\beta} \theta_{\beta}+\frac{i}{\sqrt{2}} \theta \theta \sigma_{\alpha \dot{\beta}}^{\mu} \partial_{\mu} \lambda^{\dagger \dot{\beta}}(y)
$$

where the $y$ coordinate is the same as in equation 3.8. We previously noted that the product of two chiral superfields is a chiral superfield and that a gauge invariant $F$ term of a chiral superfield is a potential Lagrangian. Indeed, the kinetic terms for the component fields can be attained as follows ${ }^{4}$

$$
\mathcal{L}=\left.\frac{1}{4}\left(\mathcal{F}^{\alpha} \mathcal{F}_{\alpha}\right)\right|_{F}=-\frac{1}{4} F_{\mu \nu} F^{\mu \nu}+i \lambda^{\dagger} \bar{\sigma}^{\mu} \partial_{\mu} \lambda+\frac{1}{2} D^{2}
$$

where the component fields are functions of the normal spacetime coordinate $x$. This is because for a chiral superfield $\Phi(y, \theta)$ we have that $\left.\Phi(y, \theta)\right|_{F}=\left.\Phi(y=x, \theta)\right|_{F}$ as the difference between $x$ and $y$ contains $\theta^{\dagger}$ and extracting the $F$ term sets $\theta^{\dagger}$ to zero (see equation 3.20). Like the $F$ field in equation 3.22, we see that the kinetic term for the $D$ field has no derivatives and therefore does not propagate. This confirms that

\footnotetext{
${ }^{4}$ The $F$ term of $\frac{1}{4} \mathcal{F}^{\alpha} \mathcal{F}_{\alpha}$ also contains the term $-\frac{i}{8} \epsilon^{\mu \nu \rho \sigma} F_{\mu \nu} F_{\rho \sigma}$. These types of terms are discussed in greater detail in the footnote containing equation 2.38. As mentioned there, it can be shown that these types of terms are total derivatives and so do not play a role in perturbation theory. We will ignore them from here on out for this reason.
} 
it is an auxiliary field.

In the non-abelian case, the proper generalization of the field-strength superfield is $\mathcal{F}_{\alpha}=\bar{D} \bar{D}\left(e^{2 g \mathcal{V}} D_{\alpha} e^{-2 g \mathcal{V}}\right)=\mathcal{F}_{\alpha}^{a} \tau^{a}$ where $\mathcal{V}=\mathcal{V}^{a} \tau^{a}$ as before. The matrices $\tau^{a}$ are the generators for a representation of the non-abelian gauge group (usually taken to be the fundamental representation if the gauge group is $\mathrm{SU}(n))$. The field-strength is again a chiral superfield but it is no longer gauge invariant. The individual superfields $\mathcal{F}_{\alpha}^{a}$ evaluated in Wess-Zumino gauge are of the same form as in equation 3.28 except each component field has an $a$ index and the derivative acting on the gaugino spinor $\lambda^{a}$ becomes a covariant derivative. A gauge invariant Lagrangian containing the kinetic terms for the component fields is then

$$
\mathcal{L}=\left.\frac{1}{4}\left(\mathcal{F}^{a \alpha} \mathcal{F}_{\alpha}^{a}\right)\right|_{F}=-\frac{1}{4} F_{\mu \nu}^{a} F^{a \mu \nu}+i \lambda^{a \dagger} \bar{\sigma}^{\mu} D_{\mu} \lambda^{a}+\frac{1}{2}\left(D^{a}\right)^{2}
$$

\subsubsection{The superpotential}

Additional supersymmetric Lagrangians can be constructed by using what is known as the superpotential. Consider first a set of chiral superfields $\Phi_{i}$. The superpotential is then a gauge invariant holomorphic function of the chiral superfields $W=W\left(\Phi_{i}\right)$. This again implies that the superpotential is itself a chiral superfield and extracting its $F$ term gives a possible Lagrangian

$$
\mathcal{L}=\left.W\right|_{F}+\text { h.c. }
$$

where we added the hermitian conjugate to make $\mathcal{L}$ real. ${ }^{5}$ The superpotential is generally a polynomial in the chiral superfields and since we are only considering

\footnotetext{
${ }^{5}$ The hermitian conjugate terms can also be obtained by first taking the hermitian conjugate of the superpotential to make it an antichiral superfield and then extracting its $\theta^{\dagger} \theta^{\dagger} / 2$ term.
} 
renormalizable Lagrangians it is at most cubic in the superfields. Possibly relevant $F$ terms are then of the form

$$
\begin{aligned}
\left.\Phi_{i}\right|_{F} & =F_{i} \\
\left.\Phi_{i} \Phi_{j}\right|_{F} & =\phi_{i} F_{j}+\phi_{j} F_{i}-\chi_{i} \chi_{j} \\
\left.\Phi_{i} \Phi_{j} \Phi_{k}\right|_{F} & =\phi_{i} \phi_{j} F_{k}+\phi_{j} \phi_{k} F_{i}+\phi_{k} \phi_{i} F_{j}-\phi_{i} \chi_{j} \chi_{k}-\phi_{j} \chi_{k} \chi_{i}-\phi_{k} \chi_{i} \chi_{j}
\end{aligned}
$$

where the fields are functions of $x$.

\subsection{Soft supersymmetry breaking terms}

Supersymmetry is not an exact symmetry of Nature as evidenced by the fact that there is no scalar electron with the same mass as the electron. Thus, supersymmetry is broken. In this section we write down terms which break supersymmetry softly (that is, terms that do not reintroduce quadratically divergent contributions to the mass squared terms of scalar fields).

Consider again a set of chiral superfields $\Phi_{i}$. The first type of terms which break supersymmetry softly are mass squared terms for the scalar components of the chiral superfields

$$
\mathcal{L}_{\text {soft }} \supset \sum_{i} m_{i}^{2}\left|\phi_{i}\right|^{2}
$$

Next, consider the superpotential for this set of chiral superfields $W=W\left(\Phi_{i}\right)$. For each term in the superpotential, we can write a soft supersymmetry breaking term which has the same form but with the superfields replaced with their scalar 
components. For example, if $W \supset y \Phi_{i} \Phi_{j} \Phi_{k}$, then

$$
\mathcal{L}_{\text {soft }} \supset a_{y} \phi_{i} \phi_{j} \phi_{k}+\text { h.c. }
$$

These types of terms are referred to as $A$ terms. If $W \supset \mu \Phi_{i} \Phi_{j}$, then

$$
\mathcal{L}_{\text {soft }} \supset b_{\mu} \phi_{i} \phi_{j}+\text { h.c. }
$$

These types of terms are referred to as $B$ terms.

The final type of soft supersymmetry breaking terms are Majorana gaugino masses. If the gaugino is a part of a non-abelian vector superfield, then it can have a soft supersymmetry breaking mass term

$$
\mathcal{L}_{\text {soft }} \supset-\frac{1}{2} m \lambda^{a} \lambda^{a}+\text { h.c. }
$$

whereas if it is a part of an abelian vector superfield, then its mass term is of the same form except without the $a$ indices.

\subsection{The scalar potential}

As noted in sections 3.4.1 and 3.4.2, the kinetic terms for the $F$ field of a chiral superfield and the $D$ field of a vector superfield do not have derivatives. It is then simple to integrate out these fields by solving for them through their equations of motion. Doing so results in contributions to the scalar potential $V$. If this procedure is done for the $F$ fields, then

$$
V \supset \sum_{j} F_{j}^{\dagger} F_{j}=\sum_{j}\left|\frac{\partial W\left(\phi_{i}\right)}{\partial \phi_{j}}\right|^{2}
$$


where $W\left(\phi_{i}\right)$ is the superpotential where each of the chiral superfields $\Phi_{i}$ have been replaced by their scalar components. Integrating out the $D$ field of an abelian vector superfield gives

$$
V \supset \frac{1}{2} D^{2}=\sum_{i, j} \frac{e^{2}}{2}\left(q_{i} \phi_{i}^{\dagger} \phi_{i}\right)\left(q_{j} \phi_{j}^{\dagger} \phi_{j}\right)
$$

Repeating this procedure for non-abelian vector superfields results in

$$
V \supset \frac{1}{2} \sum_{a} D^{a} D^{a}=\sum_{a, i, j} \frac{g^{2}}{2}\left(\phi_{i}^{\dagger} \tau_{i}^{a} \phi_{i}\right)\left(\phi_{j}^{\dagger} \tau_{j}^{a} \phi_{j}\right)
$$

where the $\tau_{i}^{a}$ are the generators of the representation in which the $\phi_{i}$ transform. The last contributions to the scalar potential are simply the soft supersymmetry breaking mass squared terms, $A$ terms, and $B$ terms as discussed in section 3.5.

\subsection{The MSSM}

As discussed in section 2.5, the SM consists of gauge bosons for the gauge group $\mathrm{SU}(3)_{\mathrm{C}} \times \mathrm{SU}(2)_{\mathrm{L}} \times \mathrm{U}(1)_{\mathrm{Y}}$, three generations of chiral fermions $Q^{i}, U^{i}, D^{i}, L^{i}$, and $E^{i}$, and a single scalar doublet $H$. We can supersymmetrize this field content by using the results presented in the previous sections. The outcome is the Minimal Supersymmetric Standard Model (MSSM).

\subsubsection{Kinetic terms}

The gauge bosons for the SM gauge group, which we denoted by $G_{\mu}^{a}$ for $\mathrm{SU}(3)_{\mathrm{C}}, W_{\mu}^{b}$ for $\mathrm{SU}(2)_{\mathrm{L}}$, and $B_{\mu}$ for $\mathrm{U}(1)_{\mathrm{Y}}$, become the gauge fields for vector superfields $\mathcal{G}^{a}, \mathcal{W}^{b}$, and $\mathcal{B}$. The fermion superpartners of the gluons, $W$ bosons, and the $B$ boson are called gluinos, $\tilde{G}$, winos, $\tilde{W}$, and the bino, $\tilde{B}$, respectively. Each vector superfield has 
an associated field-strength superfield which we denote by $\mathcal{F}_{\mathcal{G} \alpha}^{a}, \mathcal{F}_{\mathcal{W} \alpha}^{b}$, and $\mathcal{F}_{\mathcal{B} \alpha}$. The pure gauge part of the MSSM Lagrangian is then

$$
\mathcal{L}_{\text {gauge }}^{\mathrm{MSSM}}=\left.\frac{1}{4}\left(\mathcal{F}_{\mathcal{G}}^{a \alpha} \mathcal{F}_{\mathcal{G} \alpha}^{a}\right)\right|_{F}+\left.\frac{1}{4}\left(\mathcal{F}_{\mathcal{W}}^{b \alpha} \mathcal{F}_{\mathcal{W} \alpha}^{b}\right)\right|_{F}+\left.\frac{1}{4}\left(\mathcal{F}_{\mathcal{B}}^{\alpha} \mathcal{F}_{\mathcal{B} \alpha}\right)\right|_{F}
$$

The chiral fermions of the SM each become the fermionic part of chiral superfields. Recall that the fermionic part of a chiral superfield is a left-handed spinor. It is then straightforward to construct chiral superfields containing $Q^{i}$ and $L^{i}$ since both are left-handed. However, $U^{i}, D^{i}$, and $E^{i}$ are right-handed fermions. We can make these fields left-handed spinors by applying hermitian conjugates to them. We denote the resulting left-handed spinors by $\bar{U}^{i}, \bar{D}^{i}$, and $\bar{E}^{i}$. These spinors transform in the conjugate representation of the SM gauge group compared to their right-handed counterparts. Using the notation $\left(\boldsymbol{r}_{\mathbf{C}}, \boldsymbol{r}_{\mathbf{L}}, Y\right)$ to represent the gauge charges of a chiral superfield under the $\mathrm{SU}(3)_{\mathrm{C}} \times \mathrm{SU}(2)_{\mathrm{L}} \times \mathrm{U}(1)_{\mathrm{Y}}$ gauge symmetry, the MSSM contains:

- a chiral superfield $Q^{i}$ with gauge charges $(\mathbf{3}, \mathbf{2}, 1 / 6)$,

- a chiral superfield $U^{c i}$ with gauge charges $(\overline{\mathbf{3}}, \mathbf{1},-2 / 3)$,

- a chiral superfield $D^{c i}$ with gauge charges $(\overline{\mathbf{3}}, \mathbf{1}, 1 / 3)$,

- a chiral superfield $L^{i}$ with gauge charges $(\mathbf{1}, \mathbf{2},-1 / 2)$,

- a chiral superfield $E^{c i}$ with gauge charges $(\mathbf{1}, \mathbf{1}, 1)$,

where the $Q^{i}$ superfield contains the $Q^{i}$ fermions and so on. The names of the scalar components of these superfields are determined by prepending an "s" onto the corresponding SM fermion name. For example, the scalar superpartners of quarks are squarks and the scalar superpartners of leptons are sleptons. The $T_{3}=1 / 2$ fermion 
of the $Q^{3}$ superfield is the left-handed top and its scalar superpartner is the lefthanded stop, $\tilde{t}_{L}$, whereas the fermion of the $U^{c 3}$ is the right-handed top and its scalar superpartner is the right-handed stop, $\tilde{t}_{R}$. The Lagrangian for these superfields is

$$
\mathcal{L}_{\text {chiral-I }}^{\mathrm{MSSM}}=\left.\sum_{\Phi=Q, U^{c}, D^{c}, L, E^{c}} \Phi^{i \dagger} e^{-2 \mathcal{V}} \Phi^{i}\right|_{D}
$$

where the vector superfield in the exponential is dependent on the gauge charges of the chiral superfield. For example, the vector superfield for $Q$ is $\mathcal{V}=g_{s} \mathcal{G}^{a} \frac{\lambda^{a}}{2}+g \mathcal{W}^{b} \frac{\sigma^{b}}{2}+\frac{g^{\prime}}{6} \mathcal{B}$ whereas it is $\mathcal{V}=g_{s} \mathcal{G}^{a} \frac{\left(-\lambda^{a}\right)^{*}}{2}-\frac{2 g^{\prime}}{3} \mathcal{B}$ for $U^{c}$.

The Higgs content in the SM consists of a single Higgs doublet $H$. However, for reasons that will be explained shortly, the MSSM requires two Higgs doublets. Thus, the MSSM also contains

- a chiral superfield $H_{u}$ with gauge charges $(\mathbf{1}, \mathbf{2}, 1 / 2)$,

- a chiral superfield $H_{d}$ with gauge charges $(\mathbf{1}, \mathbf{2},-1 / 2)$.

The fermions of these superfields are referred to as Higgsinos and are denoted $\tilde{H}_{u}$ and $\tilde{H}_{d}$. The first reason why we need a second Higgs superfield is to avoid a $\mathrm{U}(1)_{\mathrm{Y}}$ gauge anomaly which would occur if we had, for example, only $H_{u}$ and its chiral fermion $\tilde{H}_{u}$. The Lagrangian for these superfields is then

$$
\mathcal{L}_{\text {chiral-II }}^{\mathrm{MSSM}}=\left.\sum_{\Phi=H_{u}, H_{d}} \Phi^{\dagger} e^{-2 \mathcal{V}} \Phi\right|_{D}
$$

where $\mathcal{V}=g \mathcal{W}^{b} \frac{\sigma^{b}}{2}+\frac{g^{\prime}}{2} \mathcal{B}$ for $H_{u}$ and $\mathcal{V}=g \mathcal{W}^{b} \frac{\sigma^{b}}{2}-\frac{g^{\prime}}{2} \mathcal{B}$ for $H_{d}$ 


\subsubsection{Superpotential}

The superpotential is a gauge invariant holomorphic function of the chiral superfields. Using the superfields given in the previous section, there are eight possible terms in the MSSM which have mass dimension $\leq 3$. These are

$$
W=W_{\mathrm{RPC}}+W_{\mathrm{RPV}}
$$

where

$$
\begin{aligned}
& W_{\mathrm{RPC}}=y_{u}^{i j} U^{c i} Q^{j} H_{u}-y_{d}^{i j} D^{c i} Q^{j} H_{d}-y_{e}^{i j} E^{c i} L^{j} H_{d}+\mu H_{u} H_{d}, \\
& W_{\mathrm{RPV}}=\frac{\lambda_{i j k}}{2} L^{i} L^{j} E^{c k}+\lambda_{i j k}^{\prime} L^{i} Q^{k} D^{c k}+\lambda_{i j k}^{\prime \prime} U^{c i} D^{c j} D^{c k}+\epsilon_{i} H_{u} L^{i} .
\end{aligned}
$$

The first three terms in $W_{\mathrm{RPC}}$ give masses to the SM fermions after the scalar Higgs doublets acquire vevs and break the electroweak symmetry. Notice that the holomorphicity of the superpotential necessarily implies that both $H_{u}$ and $H_{d}$ are required for all fermion masses to be generated. This is the second reason why the MSSM requires two Higgs doublets. The labels RPC and RPV stand for $R$-parity conserving and $R$-parity violating, respectively. The RPV terms are typically undesirable as they break discrete symmetries of the SM. In particular, the $\lambda, \lambda^{\prime}$, and $\epsilon$ terms break lepton number and the $\lambda^{\prime \prime}$ term breaks baryon number. These terms can be forbidden by invoking $R$-parity. This is a discrete $\mathbb{Z}_{2}$ symmetry under which the Grassmann coordinates $\theta$ and $\theta^{\dagger}$ are odd, the chiral superfields $Q, U^{c}, D^{c}, L$, and $E^{c}$ are odd, and the chiral superfields $H_{u}$ and $H_{d}$ are even. The four terms in $W_{\mathrm{RPC}}$ are then even under $R$-parity while the four terms in $W_{\mathrm{RPV}}$ are odd. Requiring the superpotential to be even then removes $W_{\mathrm{RPV}}$. If we assign the vector superfields $\mathcal{G}^{a}, \mathcal{W}^{b}$, and $\mathcal{B}$ to 
be even, then the $R$-parity charge of any particle is given by

$$
R=(-1)^{3(B-L)+2 s}
$$

where $B, L$, and $s$ are the baryon number, lepton number, and spin of the particle, respectively. The SM gauge bosons and fermions and the two Higgs doublets are then even whereas their superpartners are odd. Additionally, conservation of $R$ parity significantly affects supersymmetry phenomenology. For example, if $R$-parity is conserved, then supersymmetric particles must be produced in pairs at particle colliders. Furthermore, $R$-parity conservation also requires unstable supersymmetric particles to decay into final states with an odd number of lighter supersymmetric particles. This implies that the lightest supersymmetric particle (LSP) is stable and can therefore be a potential dark matter candidate. Thus, it is important to distinguish whether $R$-parity is conserved, and so we note that the MSSM usually assumes that it is. On the other hand, when the $R$-parity violating terms are included, the resulting model is called the $R$-Parity Violating Minimal Supersymmetric Standard Model (RPVMSSM). A comprehensive review of the RPVMSSM is given in [19].

\subsubsection{Soft supersymmetry breaking terms}

The MSSM Lagrangian also contains soft supersymmetry breaking terms. These can be determined by applying the results in section 3.5 to the MSSM. The first type are soft mass squared terms for the scalar components of the chiral superfields. The Lagrangian for these terms is then

$$
\begin{aligned}
\mathcal{L}_{\text {soft-m }}^{\mathrm{MSSM}}= & -\left(m_{Q}^{2}\right)_{i j} \tilde{Q}^{i \dagger} \tilde{Q}^{j}-\left(m_{U}^{2}\right)_{i j} \tilde{U}^{c i \dagger} \tilde{U}^{c j}-\left(m_{D}^{2}\right)_{i j} \tilde{D}^{c i \dagger} \tilde{D}^{c j}-\left(m_{L}^{2}\right)_{i j} \tilde{L}^{i \dagger} \tilde{L}^{j} \\
& -\left(m_{E}^{2}\right)_{i j} \tilde{E}^{c i \dagger} \tilde{E}^{c j}-m_{H_{u}}^{2} H_{u}^{\dagger} H_{u}-m_{H_{d}}^{2} H_{d}^{\dagger} H_{d} .
\end{aligned}
$$


Arbitrary mass matrices for the scalar components of the SM fermions can result in large contributions to highly constrained flavour violating processes. Thus, the matrices $m_{Q}^{2}, m_{U}^{2}, m_{D}^{2}, m_{L}^{2}$, and $m_{E}^{2}$ should be nearly diagonal in flavour space. This can be accommodated provided they are generated in a flavour blind manner. Additionally, the soft mass squared terms for the Higgs doublets $H_{u}$ and $H_{d}$ play an important role in electroweak symmetry breaking.

The next type are $A$ and $B$ terms that are of the same form as the superpotential but with the superfields replaced by their scalar components. The Lagrangian for these terms is then

$$
\mathcal{L}_{\mathrm{soft}-A, B}^{\mathrm{MSSM}}=-\left(a_{u}\right)_{i j} \tilde{U}^{c i} \tilde{Q}^{j} H_{u}+\left(a_{d}\right)_{i j} \tilde{D}^{c i} \tilde{Q}^{j} H_{d}+\left(a_{e}\right)_{i j} \tilde{E}^{c i} \tilde{L}^{j} H_{d}-B_{\mu} H_{u} H_{d}+\text { h.c. }
$$

where we have only written $A$ and $B$ terms for the RPC terms in the superpotential. If $R$-parity is violated, then $A$ and $B$ terms for the RPV terms in the superpotential should also be included. It is common to introduce a notation where the $A$ terms are scaled by their respective Yukawa couplings. For example, instead of using $\left(a_{u}\right)_{33} \equiv$ $a_{t}$, the parameter $A_{t}$ is often used where $a_{t}=A_{t} y_{t}$.

Finally, there are also soft masses for the gauginos

$$
\mathcal{L}_{\text {soft-gaugino }}^{\mathrm{MSSM}}=-\frac{1}{2} M_{3} \tilde{G}^{a} \tilde{G}^{a}-\frac{1}{2} M_{2} \tilde{W}^{b} \tilde{W}^{b}-\frac{1}{2} M_{1} \tilde{B} \tilde{B}+\text { h.c. }
$$

This completes the MSSM Lagrangian. 


\subsubsection{Electroweak symmetry breaking}

The entire scalar potential of the MSSM is quite complicated. There is the $F$ term contribution of the form given by equation 3.37, the $D$ term contribution of the form given in equation 3.38 for $\mathrm{U}(1)_{\mathrm{Y}}$ and 3.39 for $\mathrm{SU}(2)_{\mathrm{L}}$ and $\mathrm{SU}(3)_{\mathrm{C}}$, and finally the soft supersymmetry breaking terms given in equations 3.47 and 3.48. Phenomenologically, colour and electric charge are unbroken symmetries of the vacuum and this signifies that the scalar particles charged under these symmetries do not acquire vevs. In fact, this thesis will make use of the absence of colour and charge breaking vacua to constrain different combinations of parameters.

The scalar particles which acquire vevs are the neutral components of the Higgs doublets $H_{u}$ and $H_{d}$. We denote these vevs by $\left\langle H_{u}^{0}\right\rangle=v_{u}$ and $\left\langle H_{d}^{0}\right\rangle=v_{d}$ where both $v_{u}$ and $v_{d}$ are real and positive. These vevs break the electroweak gauge group $\mathrm{SU}(2)_{\mathrm{L}} \times \mathrm{U}(1)_{\mathrm{Y}}$ to the subgroup $\mathrm{U}(1)_{\mathrm{EM}}$ as in SM. Subbing the vevs into the kinetic terms for the doublets $H_{u}$ and $H_{d}$ generates masses for the $W$ and $Z$ bosons in a similar fashion as in section 2.6. Performing the calculation shows that the generated masses are the same provided the equality $v^{2}=v_{u}^{2}+v_{d}^{2}$ holds where $v$ is the vev of the SM Higgs doublet. Instead of using the vevs $v_{u}$ and $v_{d}$, it is often more convenient to use $v$ and $\tan \beta$ where

$$
\tan \beta=\frac{v_{u}}{v_{d}} .
$$

After electroweak symmetry breaking, particles with different electroweak charges but the same electric charges can potentially mix. Such mixing occurs in the Higgs 
sector between the two Higgs doublets. For the neutral Higgs particles, we can write

$$
\left(\begin{array}{c}
H_{u}^{0} \\
H_{d}^{0}
\end{array}\right)=\left(\begin{array}{c}
v_{u} \\
v_{d}
\end{array}\right)+\frac{1}{\sqrt{2}} R_{\alpha}\left(\begin{array}{c}
h^{0} \\
H^{0}
\end{array}\right)+\frac{i}{\sqrt{2}} R_{\beta}\left(\begin{array}{c}
G^{0} \\
A^{0}
\end{array}\right)
$$

In this expression, $h^{0}$ and $H^{0}$ are light and heavy CP-even scalars, $A^{0}$ is a CP-odd scalar, and $G^{0}$ is the Goldstone boson which becomes the longitudinal mode of the $Z$ boson. The rotation matrices are

$$
R_{\alpha}=\left(\begin{array}{cc}
\cos \alpha & \sin \alpha \\
-\sin \alpha & \cos \alpha
\end{array}\right) \quad \text { and } \quad R_{\beta}=\left(\begin{array}{cc}
\sin \beta & \cos \beta \\
-\cos \beta & \sin \beta
\end{array}\right)
$$

The rotation matrix $R_{\beta}$ is also the matrix that appears for the charged Higgs doublets

$$
\left(\begin{array}{c}
H_{u}^{+} \\
H_{d}^{-\dagger}
\end{array}\right)=R_{\beta}\left(\begin{array}{l}
G^{+} \\
H^{+}
\end{array}\right)
$$

where $H^{+}$and its complex conjugate $H^{-}$are the charged Higgs bosons and $G^{+}$ and its complex conjugate $G^{-}$are the charged Goldstone bosons which become the longitudinal modes of the $W^{ \pm}$bosons. The tree-level masses of the five physical scalar fields are

$$
\begin{aligned}
m_{A^{0}}^{2} & =\frac{2 B_{\mu}}{\sin (2 \beta)} \\
m_{h^{0}, H^{0}}^{2} & =\frac{1}{2}\left(m_{A^{0}}^{2}+m_{Z}^{2} \mp \sqrt{\left(m_{A^{0}}^{2}-m_{Z}^{2}\right)^{2}+4 m_{Z}^{2} m_{A^{0}}^{2} \sin ^{2}(2 \beta)}\right) \\
m_{H^{ \pm}}^{2} & =m_{A^{0}}^{2}+m_{W}^{2} .
\end{aligned}
$$


The mixing angle $\alpha$ is determined at tree-level by the relations

$$
\frac{\sin 2 \alpha}{\sin 2 \beta}=-\left(\frac{m_{H^{0}}^{2}+m_{h^{0}}^{2}}{m_{H^{0}}^{2}-m_{h^{0}}^{2}}\right), \quad \frac{\tan 2 \alpha}{\tan 2 \beta}=\left(\frac{m_{A^{0}}^{2}+m_{Z}^{2}}{m_{A^{0}}^{2}-m_{Z}^{2}}\right)
$$

Finally, from the formula for $m_{h^{0}}^{2}$ in equation 3.54, it can be shown that at tree-level $m_{h^{0}}<m_{Z}|\cos (2 \beta)|<m_{Z}$. Large radiative corrections to the Higgs mass are therefore needed to reconcile this inequality with the $125 \mathrm{GeV}$ mass of the Higgs boson.

Masses for the SM fermions are generated in a very similar fashion as in section 2.6 with one important difference: the vev $v$ appearing in equation 2.57 is replaced with $v_{u}$ for the up-type quarks and $v_{d}$ for the down-type quarks and charged leptons. The fermion masses in the MSSM are then of the form

$$
m_{u}=y_{u} v_{u}=y_{u} v \sin \beta, \quad m_{d}=y_{d} v_{d}=y_{d} v \cos \beta, \quad m_{e}=y_{e} v_{e}=y_{e} v \cos \beta .
$$

This implies that the up-type Yukawa couplings are enhanced compared to their SM counterparts by $(\sin \beta)^{-1}$ and the down-type and lepton Yukawa couplings are enhanced compared to their SM counterparts by $(\cos \beta)^{-1}$. This latter enhancement can be quite significant in the large $\tan \beta$ regime.

Another significant implication of electroweak symmetry breaking is mixing between the neutral wino, the bino, and the neutral Higgsinos. The resulting mass eigenstates are called neutralinos. Similarly, the charged winos also mix with the charged Higgsinos resulting in mass eigenstates called charginos. We forgo showing the mixing matrices here as they will be presented in detail in chapter 5, specifically equations 5.3 and 5.5. Also significant is the mixing between left-handed and right-handed squarks and sleptons after electroweak symmetry breaking. Specifically 
relevant for this thesis is the mixing between the left- and right-handed stops, $\tilde{t}_{L}$ and

$\tilde{t}_{R}$, to form mass eigenstates $\tilde{t}_{1}$ and $\tilde{t}_{2}$ and the mixing between the left- and righthanded sbottoms, $\tilde{b}_{L}$ and $\tilde{b}_{R}$, to form mass eigenstates $\tilde{b}_{1}$ and $\tilde{b}_{2}$. We again forgo showing the mixing matrices here as they will also be presented in detail in chapter 5, specifically equations 5.25 and 5.26 .

\subsection{Mediation mechanisms}

If supersymmetry is realized by the Lagrangian of Nature, then it must be broken spontaneously. This implies that the vacuum is not invariant under supersymmetry transformations, so that $Q_{\alpha}|0\rangle \neq 0$ and $Q_{\dot{\alpha}}^{\dagger}|0\rangle \neq 0$. From the algebra in equation 3.1 it can be shown that the Hamiltonian $H=P^{0}$ is equal to $H=(1 / 2)\left(Q_{1} Q_{1}^{\dagger}+Q_{1}^{\dagger} Q_{1}+\right.$ $\left.Q_{2} Q_{2}^{\dagger}+Q_{2}^{\dagger} Q_{2}\right)$. Computing the vacuum expectation value then implies

$$
\langle 0|H| 0\rangle=\langle 0|V| 0\rangle=\frac{1}{2}\left(\| Q_{1}^{\dagger}|0\rangle\left\|^{2}+\right\| Q_{1}|0\rangle\left\|^{2}+\right\| Q_{2}^{\dagger}|0\rangle\left\|^{2}+\right\| Q_{2}|0\rangle \|^{2}\right)
$$

where $V$ is the scalar potential and we have ignored any spacetime dependent and fermion condensate effects when setting $\langle 0|H| 0\rangle=\langle 0|V| 0\rangle$. Thus, broken supersymmetry is equivalent to the scalar potential having a non-zero vev. As discussed in section 3.6, the scalar potential comes from integrating out the $F$ terms of chiral superfields and the $D$ terms of vector superfields. It follows that if an $F$ term or a $D$ term acquire a vev, then supersymmetry will be spontaneously broken.

In section 3.7.3, we wrote down the soft supersymmetry breaking terms appearing in the MSSM. However, these types of terms cannot be generated within the MSSM. This is because the supertrace of a spontaneously broken supersymmetric 
theory with only renormalizable terms satisfies the following property

$$
\mathrm{S} \operatorname{Tr}\left(m^{2}\right)=\sum_{j}(-1)^{2 j}(2 j+1) \operatorname{Tr}\left(m_{j}^{2}\right)=0
$$

where the sum includes all particles with spins $j$ and tree-level masses $m_{j}$. Clearly, this condition does not hold in the MSSM as it would imply scalars with masses less than the known fermion masses, which is ruled out by experiments. Thus, to generate the soft supersymmetry breaking terms in the MSSM, it is necessary to introduce a hidden sector where supersymmetry is spontaneously broken and then to communicate this breaking to the MSSM in some way. This is known as mediation and we discuss the following three types: gravity mediation, gauge mediation [20-25], and anomaly mediation $[26,27]$.

\subsubsection{Gravity mediation}

In gravity mediated supersymmetry breaking, the $F$ term of some chiral superfield $X$ in the hidden sector acquires a vev $\langle F\rangle$ and thus breaks supersymmetry. This breaking is then communicated to the MSSM fields radiatively by new physics, including gravity, which resides at the Planck scale. This results in non-renormalizable operators which give the MSSM soft supersymmetry breaking terms after replacing $X$ by its $F$ term vev (i.e. $X \rightarrow\langle F\rangle \theta^{2} / 2$ ). These non-renormalizable operators take the form

$$
\begin{aligned}
\mathcal{L}_{\mathrm{soft}-m^{2}}^{\mathrm{MSSM}}=- & \int d^{4} \theta \frac{X^{\dagger} X}{m_{P l}^{2}}\left(\left(\hat{m}_{Q}^{2}\right)_{i j} Q^{i \dagger} Q^{j}+\left(\hat{m}_{U}^{2}\right)_{i j} U^{c i \dagger} U^{c j}+\left(\hat{m}_{D}^{2}\right)_{i j} D^{c i \dagger} D^{c j}\right. \\
& \left.+\left(\hat{m}_{L}^{2}\right)_{i j} L^{i \dagger} L^{j}+\left(\hat{m}_{E}^{2}\right)_{i j} E^{c i \dagger} E^{c j}+\hat{m}_{H_{u}}^{2} H_{u}^{\dagger} H_{u}+\hat{m}_{H_{d}}^{2} H_{d}^{\dagger} H_{d}\right) \\
\mathcal{L}_{\mathrm{soft}-A, B}^{\mathrm{MSSM}}=- & \int d^{2} \theta \frac{X}{m_{P l}}\left(\left(\hat{a}_{u}\right)_{i j} U^{c i} Q^{j} H_{u}-\left(\hat{a}_{d}\right)_{i j} D^{c i} Q^{j} H_{d}-\left(\hat{a}_{e}\right)_{i j} E^{c i} L^{j} H_{d}\right)
\end{aligned}
$$




$$
\begin{aligned}
& -\int d^{4} \theta \frac{X^{\dagger} X}{m_{P l}^{2}} \hat{B}_{\mu} H_{u} H_{d}+\text { h.c. } \\
\mathcal{L}_{\text {soft-gaugino }}^{\text {MSSM }}= & -\int d^{2} \theta \frac{X}{4 m_{P l}}\left(\hat{M}_{3} \mathcal{F}_{\mathcal{G}}^{a \alpha} \mathcal{F}_{\mathcal{G} \alpha}^{a}+\hat{M}_{2} \mathcal{F}_{\mathcal{W}}^{b \alpha} \mathcal{F}_{\mathcal{W} \alpha}^{b}+\hat{M}_{1} \mathcal{F}_{\mathcal{B}}^{\alpha} \mathcal{F}_{\mathcal{B} \alpha}\right)+\text { h.c. }
\end{aligned}
$$

where the hatted terms are dimensionless. One potential issue of gravity mediated supersymmetry breaking is that it does not enforce flavour blindness on the generated terms, a desirable property as outlined in section 3.7.3. That the terms are flavour blind is simply an assumption for this mediation mechanism. Indeed, it is customary to make various simplifying assumptions regarding the forms of the generated terms. Doing so results in simpler models, including one particularly famous model known as minimal supergravity (MSUGRA) [28].

\subsubsection{Gauge mediation}

In models of gauge mediation, supersymmetry breaking is transmitted to the MSSM from the hidden sector by gauge interactions. More precisely, there is a set of heavy messenger chiral superfields which both couple to the source of supersymmetry breaking and are also charged under the SM gauge groups. Since they couple to the source of supersymmetry breaking, the scalar and fermionic components of the messenger chiral superfields have different masses. The soft supersymmetry breaking terms in the MSSM are then generated by radiative corrections. For example, figure 3.2a shows how gaugino masses can be generated at one loop while figures $3.2 \mathrm{~b}$ and $3.2 \mathrm{c}$ show example two loop diagrams (there are a few others) contributing to the generation of a mass squared term for a MSSM scalar. These types of diagrams generate masses of the form

$$
m_{\text {gaugino }} \sim \frac{g^{2}}{16 \pi^{2}} \frac{\langle F\rangle}{m_{\text {mess }}} \quad \text { and } \quad m_{\text {scalar }}^{2} \sim\left(\frac{g^{2}}{16 \pi^{2}}\right)^{2}\left(\frac{\langle F\rangle}{m_{\text {mess }}}\right)^{2}
$$




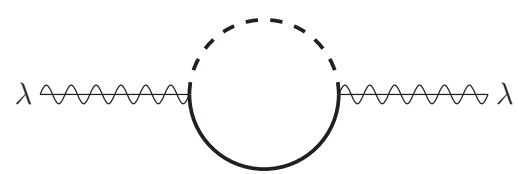

(a)

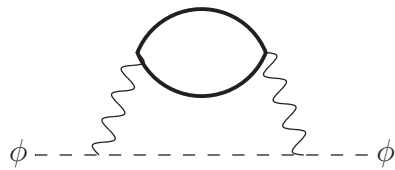

(b)

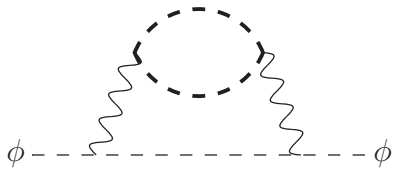

(c)

Figure 3.2: Example diagrams generating MSSM gaugino (3.2a) and scalar (3.2b and 3.2c) soft supersymmetry breaking mass terms from gauge mediation. In these diagrams, $\lambda$ and $\phi$ represent MSSM gauginos and scalars, respectively, and wavy lines represent SM gauge bosons. Additionally, the thick solid and dashed lines represent the heavy fermionic and scalar messengers, respectively.

where $g$ represents any SM gauge coupling, $\langle F\rangle$ is the supersymmetry breaking energy scale, $m_{\text {mess }}$ is the messenger mass scale, and we have suppressed numerical factors coming from, for example, group theory. Notice that $m_{\text {gaugino }} \approx m_{\text {scalar }}$ in this very minimal setup. Also, $A$ terms arise at two loops and are very small. One phenomenological advantage of gauge mediation is that it is automatically flavour blind.

\subsubsection{Anomaly mediation}

To explain anomaly mediated supersymmetry breaking, it is first necessary to promote global supersymmetry to local supersymmetry and consider supergravity. Supergravity theories exhibit the anomalous violation of superconformal invariance (also called the super-Weyl anomaly). The effects of this anomaly can be encoded by the conformal compensator field $\Phi$ whose scalar component field obtains a vacuum expectation value $\langle\Phi\rangle=1$. Further, this field couples to the source of supersymmetry breaking, parameterized by $\langle F\rangle$, and this causes its auxiliary field to also acquire a vacuum expectation value given by

$$
\left\langle F_{\Phi}\right\rangle \sim \frac{\langle F\rangle}{m_{P l}} \sim m_{3 / 2}
$$


where $m_{3 / 2}$ is the gravitino mass. Thus, we can write $\langle\Phi\rangle=1-m_{3 / 2} \theta^{2} / 2$. Anomaly mediation then arises because the conformal compensator field couples to all sources of scale invariance violation. Moreover, unlike gravity and gauge mediation, the resulting contributions are calculable within the low energy theory. For example, if scale invariance is broken by the beta function $\beta_{g}$ for a gauge couplings $g{ }^{6}$ then anomaly mediation generates an unavoidable soft supersymmetry breaking gaugino mass term $\mathcal{L} \supset-\frac{1}{2} m \lambda \lambda$ with $m$ equal to

$$
m=\frac{\beta_{g}}{g} m_{3 / 2}
$$

In the MSSM, this results in masses

$$
M_{1}=\frac{33 g_{1}^{2}}{80 \pi^{2}} m_{3 / 2}, \quad M_{2}=\frac{g_{2}^{2}}{16 \pi^{2}} m_{3 / 2}, \quad M_{3}=-\frac{3 g_{3}^{2}}{16 \pi^{2}} m_{3 / 2}
$$

where $M_{1}, M_{2}$, and $M_{3}$ are the bino, wino, and gluino masses as defined in equation 3.49 and $g_{1}=\sqrt{5 / 3} g^{\prime}, g_{2}=g$, and $g_{3}=g_{s}$. Similarly, soft mass squared terms for the scalar fields and $A$ terms are also generated due to the scale dependence of the wave-function renormalizations of the superfields and the scale dependence of the Yukawa couplings, respectively.

\footnotetext{
${ }^{6}$ The beta function $\beta$ for a generic coupling $\lambda$ determines how $\lambda$ scales with energy by appearing on the right-hand side of the renormalization group equation $d \lambda / d t=\beta$ where $t=\log \left(Q / Q_{0}\right), Q$ is the renormalization scale, and $Q_{0}$ is a reference energy scale. See, for example, appendix A for several one loop beta functions.
} 


\section{$3.9 R$-symmetries}

In section 3.7.2, the concept of $R$-parity was introduced. This is a discrete $\mathbb{Z}_{2}$ symmetry under which, for example, $\theta$ and $\theta^{\dagger}$ are odd

$$
\theta \rightarrow-\theta \quad \text { and } \quad \theta^{\dagger} \rightarrow-\theta^{\dagger}
$$

It is possible to promote this discrete $\mathbb{Z}_{2}$ symmetry to a continuous $\mathrm{U}(1)_{R}$ symmetry and require supersymmetric Lagrangians to be invariant under an $R$-symmetry transformation. The defining feature of an $R$-symmetry is that $\theta$ and $\theta^{\dagger}$ have charges +1 and -1 , respectively. That is, under a transformation parameterized by $\alpha$, these Grassmann coordinates transform

$$
\theta \rightarrow e^{i \alpha} \theta \quad \text { and } \quad \theta^{\dagger} \rightarrow e^{-i \alpha} \theta^{\dagger}
$$

which is the generalization of equation 3.66. As can be seen from their derivative operator representations given in equation 3.3, the supercharges $Q$ and $Q^{\dagger}$ then have $R$-charges -1 and +1 , respectively. Since $Q$ and $Q^{\dagger}$ interchange the on-shell scalar and fermionic states within a given supermultiplet, it follows that these types of particles have $R$-charges which differ by one unit.

In a $R$-symmetric theory, each superfield $\mathcal{S}$ will have an $R$-charge $r_{\mathcal{S}}$ so that under an $R$-transformation $\mathcal{S} \rightarrow e^{i r_{\mathcal{S}} \alpha} \mathcal{S}$. Consider the case where the superfield is a

chiral superfield $\Phi$ as given in equation 3.10. If $\Phi \rightarrow e^{i r_{\Phi} \alpha} \Phi$, then it follows that the individual component fields transform

$$
\phi \rightarrow e^{i r_{\Phi} \alpha} \phi, \quad \chi \rightarrow e^{i\left(r_{\Phi}-1\right) \alpha} \chi, \quad F \rightarrow e^{i\left(r_{\Phi}-2\right) \alpha} F .
$$


In contrast, vector superfields $\mathcal{V}$ are real and thus cannot possess a non-zero $R$ charge, i.e. $\mathcal{V} \rightarrow \mathcal{V}$. By examining the form of a vector superfield in Wess-Zumino gauge, equation 3.16, it follows that its component fields transform

$$
A_{\mu} \rightarrow A_{\mu}, \quad \lambda \rightarrow e^{i \alpha} \lambda, \quad D \rightarrow D .
$$

Notice that the gaugino $\lambda$ necessarily carries $R$-charge +1 . This feature implies that the standard Majorana mass terms for gauginos (equation 3.36 in general and equation 3.49 in the MSSM) are forbidden in an $R$-invariant theory. Since massless charged winos clearly do not exist, it is imperative to determine some other way of giving masses to the MSSM gauginos in this scenario. This can be accomplished by introducing chiral superfields $O^{a}, T^{b}$, and $S$ where $O^{a}$ transforms in the adjoint representation of $\mathrm{SU}(3)_{\mathrm{C}}, T^{b}$ transforms in the adjoint representation of $\mathrm{SU}(2)_{\mathrm{L}}$, and $S$ is a gauge singlet. If each of these new chiral superfields have $R$-charge 0 , then their fermionic components have $R$-charge -1 as shown by equation 3.68. Gauge and $R$-invariant Dirac mass terms involving the MSSM gauginos and the fermions of these chiral superfields then take the form

$$
\mathcal{L} \supset-M_{3}^{D} \tilde{G}^{a} \tilde{O}^{a}-M_{2}^{D} \tilde{W}^{b} \tilde{T}^{b}-M_{1}^{D} \tilde{B} \tilde{S}+\text { h.c. }
$$

Let us now reconsider the procedures used to construct supersymmetrically invariant Lagrangians and account for the presence of an $R$-symmetry. First, notice that the integrals in equation 3.19 imply that the integration measures $d^{2} \theta$ and $d^{2} \theta d^{2} \theta^{\dagger}$ have $R$-charges -2 and 0 , respectively. Thus, a superfield must have $R$-charge $+2(0)$ if its $F(D)$ term is to be projected out to form a supersymmetric Lagrangian. As shown in equation 3.5, the derivative operators $D_{\alpha}$ and $\bar{D}_{\dot{\alpha}}$ have $R$-charges -1 and +1 , 
respectively. It then follows that field-strength superfields have $R$-charge +1 . This can also be seen by examining the field-strength superfields in Wess-Zumino gauge, equation 3.28. The kinetic terms for vector superfields as given by equations 3.29 and 3.30 are therefore $R$-invariant. Additionally, terms of the form $\Phi^{\dagger} e^{-2 g \mathcal{V}} \Phi$ have $R$-charge 0 and therefore the kinetic terms for chiral superfields are also $R$-invariant. Finally, possible terms in the superpotential $W$ must have $R$-charge +2 .

In addition to removing the standard Majorana gaugino mass terms, the presence of an $R$-symmetry also affects the soft supersymmetry breaking $A$ and $B$ terms. The standard procedure of replacing the superpotential terms by there scalar components no longer works as the resulting soft terms would have $R$-charge +2 . Instead, the $A$ and $B$ terms are now holomorphic and gauge invariant combinations of the scalar components of chiral superfields with net zero $R$-charge. Alternatively, these new types of $A$ and $B$ terms can be thought of as coming from the standard procedure applied to otherwise allowable superpotential operators which are forbidden only because they have zero net $R$-charge.

Further steps are required to construct a realistic supersymmetric theory that possesses an $R$-symmetry. For example, the Higgs superfields must both have $R$ charge 0 to avoid spontaneously breaking the $R$-symmetry after the two Higgs doublets acquire vevs. But this implies that the $\mu$ term in the MSSM superpotential, equation 3.44 , is forbidden by the $R$-symmetry. Thus, additional model building is required to avoid massless Higgsinos. We do not do this here but instead postpone this endeavour to chapter 6 . There, an $R$-symmetric model will be constructed where the $R$-symmetry will be identified with baryon number. Interestingly, the $R$-parity violating superpotential operator $\lambda^{\prime \prime} U^{c} D^{c} D^{c}$ is permitted in the superpotential and is now baryon number conserving. 


\section{Chapter 4}

\section{Phenomenology of Mini-Split}

\section{supersymmetry}

\subsection{Introduction}

Supersymmetry was introduced in chapter 3 as a potential cure for the hierarchy problem. However, in order to do so, the superpartners of the SM particles are required to have masses not much larger than the weak scale. Even though weak scale SUSY is by no means ruled out, lack of results at the LHC suggests that the Higgs mass might be fine-tuned to a certain degree. This is the case in Split-SUSY models [29-31] where fermion superpartners can still be close to the electroweak scale while the scalar superpartners are much heavier. These theories are no longer solutions to the hierarchy problem (which could be explained by an environmental selection principle for example), but maintain a dark matter candidate and can keep intact gauge coupling unification [29-31]. However, it was shown in [32] that scalars heavier than $10^{5} \mathrm{TeV}$ would make it difficult to reconcile Split-SUSY with the known mass of the Higgs boson [33,34], therefore putting an upper limit on this splitting. 
These Split-SUSY theories with only a small gap are referred to as Mini-Split [32].

One of the main phenomenological characteristics of Mini-Split models is the presence of a small hierarchy between the gauginos and the scalars. The conventional gaugino mass spectra associated to well-known mediation mechanisms like anomaly mediation $[26,27]$ and gauge mediation [20-25] are then modified, as the heavy superpartners deflect the gaugino masses from their standard renormalization group (RG) trajectories when they are integrated out. The resulting spectra are referred to as deflected anomaly mediation $[35,36]$ or deflected gauge mediation. The precise phenomenology of Mini-Split models depends on the value of the Higgsino mass parameter $\mu$, initially defined in equation 3.44 , which could either be at the electroweak scale or at the same scale as the scalars. In this chapter, we focus on the case of large $\mu$. The case of small $\mu$ was considered in [37] which provides future prospects for anomaly mediation in Mini-Split theories at a $100 \mathrm{TeV}$ collider with light Higgsinos (which minimizes the amount of deflection) and applies these results to gauge and mirror mediation. Reference [37] also studied cases with a large $\mu$ (50 TeV) but still somewhat smaller than what is considered in most of the parameter space we consider. Dark matter predictions for such models are presented in [38,39]. Other variants of deflected mediation are studied in [40-48].

The purpose of this chapter is to constrain the parameter space of Mini-Split models with deflected anomaly and gauge mediation using LHC data and to predict future exclusion and discovery prospects at LHC 14 and a future $100 \mathrm{TeV}$ proton proton collider. Current constraints are extracted from ATLAS [49-53] and CMS [54-56] SUSY searches (mainly gluino pair production), the known mass of the Higgs boson $[33,34]$, and the absence of a colour-breaking vacuum [36]. Future prospects for LHC 14 and a $100 \mathrm{TeV}$ collider are obtained by using the same theoretical tools 
in conjunction with background estimates. In the cases studied here, the deflection comes mainly from the Higgsino sector $[36,57]$, which is assumed to be around the scalar scale and the light neutralinos/charginos are almost pure gauginos. As one generally expects the third generation of squarks to be lighter because of renormalization group effects for example, this chapter makes the simplifying assumption of a slightly lighter third generation.

This chapter is organized as follows. The necessary theoretical elements are presented first. This includes an explanation of how Mini-Split theories can arise in both anomaly and gauge mediation, as well as pole mass expressions and branching fractions. The procedure necessary to calculate the Higgs mass is also presented. The methodology used in obtaining both current limits and future prospects is then explained. This includes the LHC searches used to determine current limits. Finally, we present current LHC constraints and prospects at LHC 14 and a future $100 \mathrm{TeV}$ collider.

\subsection{Theory}

\subsubsection{Mini-Split models}

In this section, we review how Mini-Split spectra can be realized in both anomaly

and gauge mediation (see for example [36]). Quite generally, sfermion masses can be generated via terms of the form

$$
\int d^{4} \theta \frac{X^{\dagger} X}{M_{*}^{2}} Q^{\dagger} Q
$$


where $M_{*}$ is the mediation scale, $X=F_{X} \theta^{2} / 2$ is a SUSY breaking spurion, ${ }^{1}$ and $Q$ is a chiral superfield. This term is always allowed by symmetries, irrespective of the $R$-charge of $X$ or its gauge quantum numbers. On the other hand, gaugino masses are generated via terms of the form

$$
\int d^{2} \theta \frac{X}{4 M_{*}} \mathcal{F}_{i}^{\alpha} \mathcal{F}_{i \alpha}
$$

where $\mathcal{F}_{i \alpha}(i=\mathcal{B}, \mathcal{W}, \mathcal{G})$ are the MSSM field-strength superfields and we have suppressed the gauge indices. Contrary to the sfermion masses of equation 4.1, here $X$ is required to be a singlet under all gauge and global charges in order for this term to be allowed. It is therefore easier to forbid, and in the models that we consider we assume that it is absent. As initially described in greater detail in section 3.8.3, there is however an unavoidable contribution to gaugino masses coming from anomaly mediation

$$
M_{i}=\frac{\beta_{g_{i}}}{g_{i}} m_{3 / 2} .
$$

The $A$ terms are also generated by anomaly mediation and are given by

$$
A_{y}=-\frac{\beta_{y}}{y} m_{3 / 2},
$$

where $y$ is the corresponding Yukawa and $\beta_{y}$ is its beta function. A $B_{\mu}$ term can be generated by a term of the form

$$
\int d^{4} \theta \frac{X^{\dagger} X}{M_{*}^{2}} H_{u} H_{d} .
$$

\footnotetext{
${ }^{1}$ A constant which breaks a symmetry can be promoted to a dynamical field with transformation properties so that the symmetry is restored. The dynamical field is then called a spurion and can be used to parameterize the breaking of the symmetry. In this case, the $F$ term vev which breaks supersymmetry is promoted to a chiral superfield $X$ so that supersymmetrically invariant terms such as equation 4.1 can be written down.
} 
In Mini-Split scenarios, the $\mu$ term can either be large (at the scale of the scalars) or small (at the scale of the gauginos) depending on how it is generated. In this chapter we concentrate on the case where it is large, which could be generated through the Giudice-Masiero mechanism [58] where a term of the following form is introduced

$$
\int d^{4} \theta \Phi^{\dagger} \Phi\left[\hat{H}_{u, d}^{\dagger} \hat{H}_{u, d}+\left(c \hat{H}_{u} \hat{H}_{d}+\text { h.c. }\right)\right]
$$

Here $c$ is an arbitrary dimensionless constant and $\Phi$ is the conformal compensator which gets a non-zero $F$-term vev once SUSY is broken: $\Phi=1-m_{3 / 2} \theta^{2} / 2$. Upon rescaling of the fields, this becomes

$$
\int d^{4} \theta\left[H_{u, d}^{\dagger} H_{u, d}+\left(c \frac{\Phi^{\dagger}}{\Phi} H_{u} H_{d}+\text { h.c. }\right)\right]
$$

and leads to a $\mu$ term, in addition to an additional contribution to $B_{\mu}$. These terms are of order $m_{3 / 2}$ and $m_{3 / 2}^{2}$ respectively. If gravity is the sole mediator of supersymmetry breaking, then $M_{*}$ is the Planck mass and this leads to the scalars and Higgsinos all having masses of roughly $m_{3 / 2}$ while the masses of the gauginos are a loop factor smaller, leading to a Mini-Split spectrum. The fact that the $\mu$ term is taken to be large will change the running of the gauge couplings compared to the more conventional split-spectrum with light Higgsinos. The prediction for $\alpha_{s}\left(M_{Z}\right)$ was found in [57] to be smaller than with light Higgsinos, but still consistent with the measured value.

Gauge mediation can also lead to Mini-Split spectra. This can be done in a multitude of ways. We give an example taken from [32]. Assume a superpotential of the form

$$
W=M_{R}\left(\Phi_{1} \bar{\Phi}_{1}+\Phi_{2} \bar{\Phi}_{2}\right)+X \Phi_{1} \bar{\Phi}_{2}
$$

where the $\Phi_{i}$ and the $\bar{\Phi}_{i}$ are messengers and $X=M+F \theta^{2} / 2$ is a spurion that breaks 
SUSY and an $R$-symmetry. This leads to gaugino masses of

$$
M_{i}=\frac{\alpha_{i}}{6 \pi} \frac{M}{M_{R}} \frac{F^{3}}{M_{R}^{5}}+\mathcal{O}\left(\frac{M^{3}}{M_{R}^{3}} \frac{F^{3}}{M_{R}^{5}}, \frac{F^{5}}{M_{R}^{9}}\right) .
$$

On the other hand, the scalar masses are $\mathcal{O}\left(\alpha F / M_{R}\right)$. If the $R$-symmetry is weakly broken $\left(M<M_{R}\right)$, a Mini-Split spectrum is again generated.

\subsubsection{Gaugino mass spectrum}

The main effect of the small mass hierarchy between the gauginos and scalars/Higgsinos is that radiative corrections to the pole masses of gauginos coming from integrating out the scalars and Higgsinos can be comparable to, if not larger than, the contributions coming from anomaly mediation or gauge mediation directly. In the case of anomaly mediation, the expressions are well known and can be read from different sources $[36,59]$. In the limit of degenerate sfermion masses, the pole masses of the gauginos are

$$
\begin{aligned}
M_{\tilde{B}}=M_{1}(Q) & {\left[1+\frac{C_{\mu}}{11}+\frac{g_{1}^{2}}{80 \pi^{2}}\left(-\frac{41}{2} \log \frac{Q^{2}}{M_{1}^{2}}-\frac{1}{2} \log \frac{\mu^{2}}{M_{1}^{2}}+\log \frac{m_{A}^{2}}{M_{1}^{2}}\right.\right.} \\
& \left.\left.+11 \log \frac{m_{\tilde{q}}^{2}}{M_{1}^{2}}+9 \log \frac{m_{\tilde{l}}^{2}}{M_{1}^{2}}\right)+\frac{g_{3}^{2}}{6 \pi^{2}}-\frac{13 g_{t}^{2}}{264 \pi^{2} \sin ^{2} \beta}\right] \\
M_{\tilde{W}}=M_{2}(Q)[ & {\left[1+C_{\mu}+\frac{g_{2}^{2}}{16 \pi^{2}}\left(\frac{19}{6} \log \frac{Q^{2}}{M_{2}^{2}}-\frac{1}{6} \log \frac{\mu^{2}}{M_{2}^{2}}+\frac{1}{3} \log \frac{m_{A}^{2}}{M_{2}^{2}}\right.\right.} \\
& \left.\left.+3 \log \frac{m_{\tilde{q}}^{2}}{M_{2}^{2}}+\log \frac{m_{\tilde{l}}^{2}}{M_{2}^{2}}\right)+\frac{3 g_{3}^{2}}{2 \pi^{2}}-\frac{3 g_{t}^{2}}{8 \pi^{2} \sin ^{2} \beta}\right] \\
M_{\tilde{G}}=M_{3}(Q) & {\left[1+\frac{g_{3}^{2}}{16 \pi^{2}}\left(7 \log \frac{Q^{2}}{M_{3}^{2}}+4 \log \frac{m_{\tilde{q}}^{2}}{M_{3}^{2}}+13-2 F\left(\frac{M_{3}^{2}}{m_{\tilde{q}}^{2}}\right)\right)\right.} \\
& \left.-\frac{7 g_{3}^{2}}{24 \pi^{2}}+\frac{g_{t}^{2}}{12 \pi^{2} \sin ^{2} \beta}\right]
\end{aligned}
$$


where

$$
M_{1}(Q)=\frac{33 g_{1}^{2}(Q)}{80 \pi^{2}} m_{3 / 2}, \quad M_{2}(Q)=\frac{g_{2}^{2}(Q)}{16 \pi^{2}} m_{3 / 2}, \quad M_{3}(Q)=-\frac{3 g_{3}^{2}(Q)}{16 \pi^{2}} m_{3 / 2},
$$

$g_{i}(Q)$ are the gauge couplings of the $\mathrm{SM}$ in $\overline{\mathrm{MS}}$ and $\mathrm{SU}(5)$ convention at scale $Q, g_{t}$ is the top Yukawa coupling in the SM, and

$$
\begin{aligned}
C_{\mu} & =\frac{\mu}{m_{3 / 2}} \frac{m_{A}^{2} \sin ^{2} \beta}{m_{A}^{2}-\mu^{2}} \log \frac{m_{A}^{2}}{\mu^{2}} \\
F(x) & =3\left[\frac{3}{2}-\frac{1}{x}-\left(\frac{1}{x}-1\right)^{2} \log |1-x|\right] .
\end{aligned}
$$

The main point of interest is that the corrections due to $C_{\mu}$ can be comparable to, if not larger than, the usual expressions. A typical mass spectrum is shown in the left panel of figure 4.1. Similar expressions hold for gauge mediation

$$
\begin{aligned}
M_{\tilde{B}}=M_{1}^{\prime}(Q) & {\left[1+\frac{3 C_{\mu}^{\prime}}{5}+\frac{g_{1}^{2}}{80 \pi^{2}}\left(-\frac{41}{2} \log \frac{Q^{2}}{M_{1}^{2}}-\frac{1}{2} \log \frac{\mu^{2}}{M_{1}^{2}}+\log \frac{m_{A}^{2}}{M_{1}^{2}}\right.\right.} \\
& \left.\left.+11 \log \frac{m_{\tilde{q}}^{2}}{M_{1}^{2}}+9 \log \frac{m_{\tilde{l}}^{2}}{M_{1}^{2}}\right)\right] \\
M_{\tilde{W}}=M_{2}^{\prime}(Q)[ & 1+C_{\mu}^{\prime}+\frac{g_{2}^{2}}{16 \pi^{2}}\left(\frac{19}{6} \log \frac{Q^{2}}{M_{2}^{2}}-\frac{1}{6} \log \frac{\mu^{2}}{M_{2}^{2}}+\frac{1}{3} \log \frac{m_{A}^{2}}{M_{2}^{2}}\right. \\
& \left.\left.+3 \log \frac{m_{\tilde{q}}^{2}}{M_{2}^{2}}+\log \frac{m_{\tilde{l}}^{2}}{M_{2}^{2}}\right)\right] \\
M_{\tilde{G}}=M_{3}^{\prime}(Q) & {\left[1+\frac{g_{3}^{2}}{16 \pi^{2}}\left(7 \log \frac{Q^{2}}{M_{3}^{2}}+4 \log \frac{m_{\tilde{q}}^{2}}{M_{3}^{2}}+13-2 F\left(\frac{M_{3}^{2}}{m_{\tilde{q}}^{2}}\right)\right)\right.} \\
& \left.+\frac{6 g_{3}^{2}}{16 \pi^{2}}\right]
\end{aligned}
$$




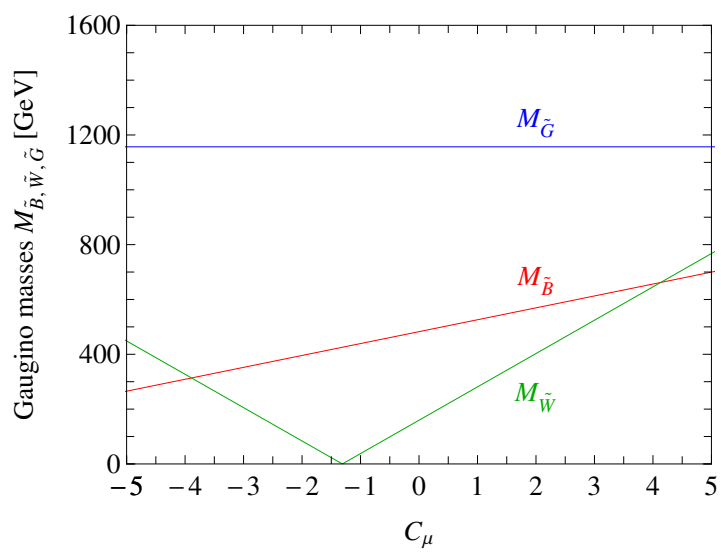

(a)

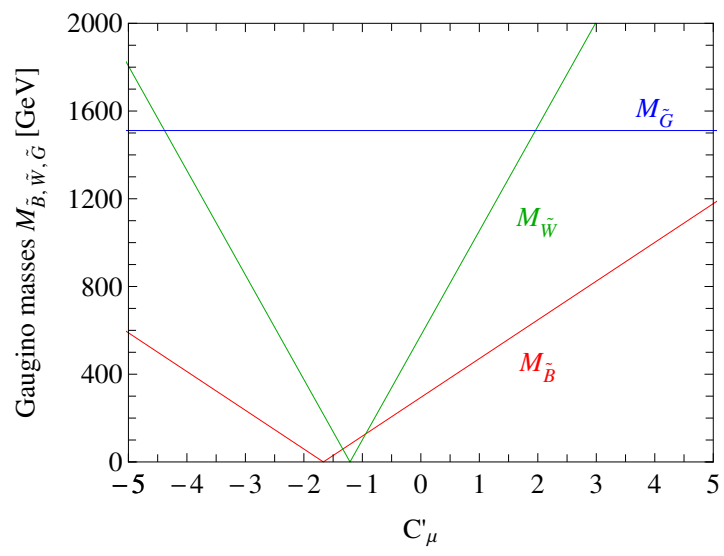

(b)

Figure 4.1: Typical mass spectrum for (a) anomaly mediation and (b) gauge mediation. In (a), the masses appearing on the right side of equation 4.10 are taken to be $m_{\text {scalars }}=\mu=m_{3 / 2}=50 \mathrm{TeV}$ with $\tan \beta=2$. In (b), the masses appearing on the right side of equation 4.13 are taken to be $m_{\text {scalars }}=\mu=\Lambda=200 \mathrm{TeV}$ with $\tan \beta=2$.

where we have kept only the terms proportional to $g_{t}, g_{3}$, or log-enhanced [60],

$$
\begin{aligned}
& M_{i}^{\prime}(Q)=\frac{g_{i}^{2}(Q)}{16 \pi^{2}} \Lambda \\
& C_{\mu}^{\prime}=\frac{\mu}{\Lambda} \frac{m_{A}^{2} \sin ^{2} \beta}{m_{A}^{2}-\mu^{2}} \log \frac{m_{A}^{2}}{\mu^{2}}
\end{aligned}
$$

where $\Lambda$, in a given gauge mediation model, can be expressed in terms of the SUSY breaking scale and the messenger scales (see for example equation 4.9). The last term of $M_{\tilde{G}}$ in equation 4.13 can be extracted from [61]. A typical mass spectrum is shown in the right panel of figure 4.1 .

The parameters $C_{\mu}$ and $C_{\mu}^{\prime}$ can be rewritten by requiring the fine-tuning condition, which needs to be imposed to have the weak scale parametrically smaller than the scalars [36]

$$
\tan ^{2} \beta=\frac{m_{H_{d}}^{2}+\mu^{2}}{m_{H_{u}}^{2}+\mu^{2}}
$$


and the usual relation $m_{A}^{2}=m_{H_{u}}^{2}+m_{H_{d}}^{2}+2 \mu^{2} . C_{\mu}$ can then be expressed as [36]

$$
C_{\mu}=\frac{2 \mu \tan \beta}{m_{3 / 2}} \frac{m_{H_{d}}^{2}+\mu^{2}}{\left(\tan ^{2} \beta+1\right) m_{H_{d}}^{2}+\mu^{2}} \log \left[\left(1+\cot ^{2} \beta\right)\left(1+\frac{m_{H_{d}}^{2}}{\mu^{2}}\right)\right] .
$$

The same applies to $C_{\mu}^{\prime}$ with $m_{3 / 2} \rightarrow \Lambda$.

In these models the gauginos are the lightest sparticles and, because $\mu$ is large, the light neutralinos and charginos are almost pure binos and winos. As such, there is a neutralino with mass very close to $M_{\tilde{B}}$ and a pair of nearly degenerate neutralino and chargino with mass $M_{\tilde{W}}$. There is a small mass difference between the neutral and charged wino dominated by a loop effect [62]

$$
\Delta M \equiv m_{\chi_{\tilde{W}}^{+}}-m_{\chi_{\tilde{W}}^{0}}=\frac{\alpha_{2} M_{2}}{4 \pi}\left[f\left(r_{W}\right)-c_{W}^{2} f\left(r_{Z}\right)-s_{W}^{2} f\left(r_{\gamma}\right)\right]
$$

where $f(y)=\int_{0}^{1}(2+2 x) \log \left(x^{2}+(1-x) y^{2}\right) d x$ and $r_{i}=m_{i} / M_{2}$. The mass splitting is typically of the order of $150 \mathrm{MeV}$.

\subsubsection{Gaugino decays}

In this chapter we concentrate on gluino decay via third generation squarks. These decay modes dominate if the third generation squarks are lighter than the others, which is expected from renormalization group effects or could be imposed for other model building reasons. ${ }^{2}$ The decays that we consider are then

$$
\begin{array}{lll}
\tilde{G} \rightarrow t \bar{t} \chi_{1}^{0} & \tilde{G} \rightarrow b \bar{b} \chi_{1}^{0} & \tilde{G} \rightarrow b \bar{t} \chi_{1}^{+} \\
\tilde{G} \rightarrow t \bar{t} \chi_{2}^{0} & \tilde{G} \rightarrow b \bar{b} \chi_{2}^{0} & \tilde{G} \rightarrow \bar{b} t \chi_{1}^{-} .
\end{array}
$$

\footnotetext{
${ }^{2}$ For example, flavour physics might require the first and second generations of squarks to be in the $1000 \mathrm{TeV}$ range, while the third generation could be kept somewhat lighter to obtain the appropriate Higgs mass [57].
} 
The gluino can also decay to a gluon and a neutralino; however, it is negligible for heavy enough Higgsinos [37] and we ignore it. To compute the branching ratios, we use analytical results that can be found in [63]. An example of branching fractions is shown in figure 4.2. In practice, $\chi_{2}^{0}$ always decays to $\chi_{1}^{0}$ and a Higgs boson [57], irrespective of whether $M_{\tilde{B}}$ is larger than $M_{\tilde{W}}$ or the opposite. In our scenario, the decay $\chi_{2}^{0}$ to $\chi_{1}^{0}$ and a $\mathrm{Z}$ boson is extremely suppressed due to the neutralinos being almost pure gauginos. When $M_{\tilde{W}}<M_{\tilde{B}}, \chi_{1}^{+}$can only decay to $\chi_{1}^{0}$ and either light leptons or a pion which can cause this chargino to be metastable because of lack of phase-space [62]. As the decay is always very soft, the decay products are generally unaccounted for and the chargino is practically indistinguishable from the stable neutralino. When $M_{\tilde{W}}>M_{\tilde{B}}, \chi_{1}^{+}$decays to $\chi_{1}^{0}$ and a $W$ boson (we verified that the decay to $\chi_{2}^{0}$ only becomes relevant for $\mu$ at a scale considerably higher than anything relevant to this chapter). The branching ratios we compute assume equal masses for the stops and the sbottoms. If the stops were lighter, the decays to two $b$ quarks, which can only proceed via off-shell sbottoms, would be relatively suppressed. As can be seen in figure 4.2 , these decays are already suppressed. The only thing that would change is the branching fraction of $\tilde{G} \rightarrow t \bar{t} \chi_{1}^{0}, \tilde{G} \rightarrow t \bar{t} \chi_{2}^{0}$, and $\tilde{G} \rightarrow b \bar{t} \chi_{1}^{+}+$h.c., which all have similar efficiencies for the searches we consider. We therefore do not expect that this assumption will affect our results greatly.

\subsubsection{Higgs mass}

To set the mass of the Higgs to its experimentally measured value, we follow the procedure outlined in [36] which we summarize here. First, $\overline{\mathrm{MS}}$ parameters are taken from reference [64] for the top Yukawa and the gauge coupling constants and from [65] for the bottom and tau Yukawas. The quartic coupling of the Higgs boson 


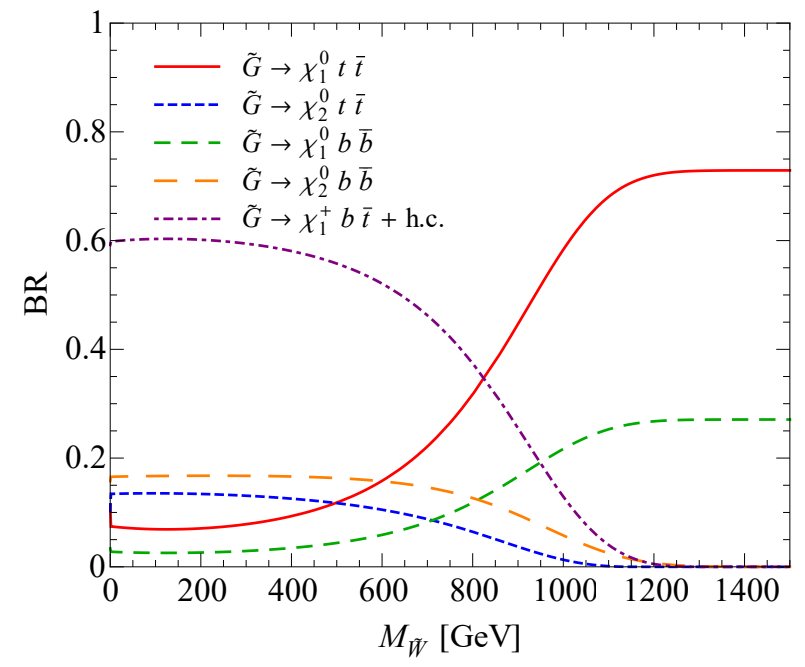

Figure 4.2: Branching ratios of the gluino for $M_{\tilde{G}}=1500 \mathrm{GeV}$ and $M_{\tilde{B}}=0 \mathrm{GeV}$. The third generation scalar masses are assumed to be degenerate and much heavier than the gauginos.

is extracted from its pole mass [66-69] using a value of $125.15 \mathrm{GeV}$, which is the naive average of the ATLAS [34] and CMS [33] values. These parameters are then evolved up to the scalars scale using three loop beta functions [70-72]. Threshold corrections are taken from [36]. These include one loop corrections and two loop QCD corrections. The Higgs quartic is then matched with its SUSY expression and the threshold corrections. This determines one of the parameters, therefore reducing the dimension of the parameter space by one. As explained in the next section, we vary $\tan \beta$ to obtain the correct value of the Higgs mass.

In some regions of the parameter space it is not possible to obtain the correct Higgs mass because the required parameters lead to a colour-breaking minimum that is deeper than the electroweak minimum. The necessary condition to avoid this is [36]

$$
\frac{\left(A_{t}-\mu \cot \beta\right)^{2}}{m_{Q_{3}} m_{U_{3}}}<\left(4-\frac{1}{\sin ^{2} \beta}\right)\left(\frac{m_{Q_{3}}^{2}}{m_{U_{3}}^{2}}+\frac{m_{U_{3}}^{2}}{m_{Q_{3}}^{2}}\right)
$$


where $m_{Q_{3}}$ is the third generation soft mass for the $\mathrm{SU}(2)_{\mathrm{L}}$ quark doublet and $m_{U_{3}}$ is the right-handed stop soft mass.

\subsection{Methodology and results}

\subsubsection{Parameter space}

We begin by discussing the parameter space we use to study the models of interest. It is very similar for both anomaly and gauge mediation. There are essentially four parameters that control the phenomenology of anomaly mediation [36]. They are $m_{3 / 2}, \tan \beta, m_{\text {scalars }}$, and $\mu$. As explained in section $4.2 .1, m_{3 / 2}$ and $m_{\text {scalars }}$ are expected to be of the same order of magnitude so we set them equal to each other. An additional parameter can be fixed by requiring the theory to predict the correct mass of the Higgs boson with the help of the results of section 4.2.4. Generally

speaking, $\tan \beta$ is the best parameter to do so as varying it even slightly can have a substantial effect on the Higgs mass. The parameter space is then reduced to $\mu$ and $m_{3 / 2}$. However, we trade $\mu$ for $C_{\mu}$. The main advantage of this parametrization is that the ratio of gaugino masses depends mostly on $C_{\mu}$. The exact details of the scalar sector are relegated to two loop corrections in equation 4.10 and our results can therefore be applied to models where the scalar sector does not differ too significantly. To translate this to something more familiar, we provide each parameter space plot with contours of constant $M_{\tilde{B}}, M_{\tilde{W}}, \mu$, and $\tan \beta$.

The relationship between $\mu$ and $C_{\mu}$ depends on $m_{H_{d}}$ which we take to be at $m_{\text {scalars. }}$ A different choice would lead, for the same $C_{\mu}$, to a different value of $\mu$ which in turn would affect mostly the colour-breaking bounds (see equation 4.19). Taking $m_{H_{d}}$ much bigger than $m_{\text {scalars }}$ would limit $C_{\mu}$ to a narrow band around 0 and taking $m_{H_{d}}$ much smaller would push the bounds to large values of $C_{\mu}$ such that the gluino 
would be the LSP for most of the parameter space. With $m_{H_{d}}$ being set to $m_{\text {scalars }}$, we have a benchmark that does not suffer from any of these drawbacks. We assume the third generation to be lighter than the others, so as a benchmark we set the first and second generation squark masses to $4 m_{\text {scalars }}$ and all third generation masses to $m_{\text {scalars }}$. This is small enough to prevent problems with large logs, while keeping the branching fraction to the first two generations below the percent level which is well below some of the uncertainties (e.g. gluino pair production cross section). Sleptons masses are also set to $4 m_{\text {scalars. }}$ Lowering the masses of the first two generations of squarks would increase the branching ratio of the gluino to light jets, possibly affecting the reach of our searches (however, the high jet-multiplicity would still provide strong bounds). It would have only a slight effect on the gaugino spectrum and on the Higgs mass. Finally, we set the third generation $A$ term $A_{t}$ by equation 4.4. Overall, changing our choice of benchmark parameters (mainly the choice of setting $m_{H_{d}}$ to $m_{\text {scalars }}$ and of taking $\left.m_{Q_{3}}=m_{U_{3}}=m_{\text {scalars }}\right)$ will mostly affect the $\mu$ and $\tan \beta$ contours in our results. Also, as a result of a modified relationship between $C_{\mu}, \mu$, and $\tan \beta$, the region of parameter space where there is a colour-breaking vacuum would also be modified.

In almost all of our parameter space the Higgsinos are heavy, except for a region near $C_{\mu}=0$ where a Higgsino can be the lightest superpartner. More precisely, outside of $\left|C_{\mu}\right|<0.3$, the Higgsinos are always an order of magnitude heavier than the gluino while only inside $\left|C_{\mu}\right|<0.1$ are the Higgsinos comparable in mass to the bino and winos. This represents only a very narrow band in the parameter space and the efficiencies of the signal regions are not expected to change much in it. In addition, this case has already been studied in $[37,73]$. As such, we neglect this effect. When $M_{\tilde{W}}<M_{\tilde{B}}$, the mass difference between $\chi_{1}^{+}$and $\chi_{1}^{0}$ is calculated using equation 
4.17.

The previous discussion applies almost directly to gauge mediation by trading $m_{3 / 2}$ for $\Lambda$. In this case, we fix $m_{\text {scalars }}$ to $\Lambda$ while $\tan \beta$ is again set by requesting the correct mass of the Higgs boson. ${ }^{3}$ The masses of the sleptons and the first two generations squarks are still set to $4 m_{\text {scalars. }} A_{t}$ is set to zero, as one would expect it to be small [36], and is then completely overshadowed by $\mu$. The mass $m_{H_{d}}$ is once more set to $m_{\text {scalars }}$.

Two other constraints are of importance for the parameter space. First of all, for a given value of $m_{3 / 2}(\Lambda)$, a small value of $A_{t}$ will lead to an upper bound on $C_{\mu}\left(C_{\mu}^{\prime}\right)$ beyond which it is impossible to obtain the correct Higgs mass. Indeed if $C_{\mu}\left(C_{\mu}^{\prime}\right)$ becomes large, the threshold corrections also become large and the quartic matching condition does not accept any solutions for real $\tan \beta$. In fact, requiring $C_{\mu}\left(C_{\mu}^{\prime}\right)$ close to its upper bound can make the Higgsinos heavy enough that large logs could become a problem and perturbation expansions could fail. Fixing the stop mixing parameter $A_{t}-\mu \cot \beta$ to a small value would solve this problem, but this would imply $A_{t}$ reaching values that are too high to be readily explained in our framework without large fine-tuning. The second issue arises from the presence of a colour-breaking vacuum which is controlled by equation 4.19 . For the values of $m_{3 / 2}$ $(\Lambda)$ considered in this chapter, it turns out that this limit is always stronger than the upper bound on $C_{\mu}\left(C_{\mu}^{\prime}\right)$ coming from the mass of the Higgs boson. This latter constraint can therefore be ignored. We limit ourselves to the regions of parameter space where equation 4.19 is satisfied.

\footnotetext{
${ }^{3}$ There is considerable freedom on the choice of the scalar masses. The choice we make is more to keep in tune with our procedure for anomaly mediation. As explained above, the exact details of the scalar sector are not very relevant in our parametrization.
} 


\begin{tabular}{|c|c|c|c|}
\hline Collaboration & Search & Strategy & Reference \\
\hline ATLAS & JHEP 06 (2014) 035 & $\begin{array}{c}2 \text { same sign / 3 } \\
\text { leptons }+0-3 \text { b-jets }+ \\
\text { MET }\end{array}$ & {$[52]$} \\
\hline ATLAS & JHEP 10 (2014) 024 & $\begin{array}{c}0-1 \text { leptons }+\geq 3 \\
\text { b-jets }+ \text { MET }\end{array}$ & {$[53]$} \\
\hline CMS & CMS-SUS-13-012 & $\begin{array}{c}\text { High jet-multiplicity } \\
+ \text { MET }\end{array}$ & {$[55]$} \\
\hline CMS & CMS-PAS-SUS-12- & $\begin{array}{c}2 \text { opposite sign } \\
\text { leptons }+ \text { high-jet } \\
\text { multiplicity }+\geq 3 \\
\text { b-jets }+ \text { MET }\end{array}$ & {$[56]$} \\
\hline
\end{tabular}

Table 4.1: Gluino pair production searches.

\subsubsection{Current LHC constraints}

To obtain current limits on anomaly and gauge mediation, we recast searches for gluino pair production. In particular, we concentrate on searches with either many b-jets, leptons, or large jet-multiplicity. Of course, all of these searches have stringent cuts on missing transverse energy (MET). The chosen searches are summarized in table 4.1. As a general rule, [53] dominates over the others. For each of these searches, we implemented codes simulating the cuts. To validate our codes, we generated events with MadGraph 5 [74] interfaced with Pythia 6.4 [75] and Delphes 3 [76,77]. We were able to reproduce all four searches with good accuracy. There are also constraints coming from electrowino production for which the experimental bounds found in $[49-51,54]$ apply directly. This is because the branching ratios for the charginos and neutralinos that are relevant for our models are the same as the one used in the simplified models considered in those searches. The bounds are in general much weaker than the ones from gluino production and become relevant only in a tiny region of parameter space where the electrowinos are very light. 
Our method to reinterpret the experimental constraints follows closely the procedure of [78]. We look at every possible combination of decay chains shown in 4.18 and evaluate for each of them the efficiency of every signal region. The branching fractions are then calculated using the procedure of section 4.2.3. The gluino pair production cross sections are calculated at NLO+NLL with NLL-fast [79-83], which we verified using Prospino [84]. The number of expected signals in a given signal region can then be calculated. The $95 \%$ confidence level signal upper limit can either be read directly from these searches or calculated using the known background and confidence level $\left(\mathrm{CL}_{\mathrm{S}}\right)$ techniques $[85,86]$. The different signal regions are combined in a boolean fashion [87]. A more thorough approach would require the correlation between the backgrounds of the different signal regions, which is not readily available.

The events are generated with MadGraph 5 [74] interfaced with Pythia 6.4 [75] and Delphes 3 [76,77]. A total of 10000 events are generated for each grid point. MadGraph generally takes care of decay chains up to the production of the LSP. The only exception is when either $\chi_{2}^{0}$ or $\chi_{1}^{+}$are very close in mass to $\chi_{1}^{0}$. These decays can then be forced to be off-shell and the decay chains become too long to be handled by MadGraph comfortably. In the worst case scenario, $\chi_{2}^{0}$ can decay to $\chi_{1}^{0}$ and an off-shell Higgs which then decays to a $W$ and an off-shell $W$ which in turn decays to other particles. To handle these difficult decays, we calculate branching ratios in advance using the decay functionalities of MadGraph to produce decay tables. $\chi_{2}^{0}$ and $\chi_{1}^{+}$are then decayed by Pythia using these results. Delphes handles the detector simulation and is tuned to simulate the ATLAS and CMS detectors.

The results for the $95 \%$ CL limits from ATLAS and CMS are given in figures 4.3 and 4.4 for anomaly and gauge mediation respectively. Each one is provided with contour plots of $M_{\tilde{B}}, M_{\tilde{W}}, \mu$, and $\tan \beta$ to relate it to more familiar parameters. The 
regions forbidden by colour-breaking vacuum are shown in purple. Overall, gluinos of mass up to $1.3 \mathrm{TeV}$ can be excluded over significant regions of parameter space. The results for the anomaly mediation spectrum can be easily understood. Over the entire covered parameter space, the gluino decays mainly to charginos. For $C_{\mu}$ between -4 and 4 , the neutral wino is the LSP. The most relevant parameter in this region is then the ratio of the mass of the LSP and of the gluino. Below $C_{\mu}$ equal to 2 , this ratio is large and the exclusion limits are strong. Above that value, the mass spectrum becomes compressed and kinematic quantities like MET become much smaller. As such, the exclusion limits drop considerably.

The results for gauge mediation are similar but with a few additional subtleties. Near $C_{\mu}^{\prime}$ equal to -5 , the spectrum is fairly compressed and the wino is too heavy to be produced. The gluino decays softly to $\chi_{1}^{0}$ and quarks, which results in lower constraints. As $C_{\mu}^{\prime}$ increases, the spectrum becomes less compressed and the limits are stronger. However, near $C_{\mu}^{\prime}$ equal to -3 , the winos become light enough to be produced and the gluino decay to chargino dominates. As these decay chains are longer, there is less MET and the constraints are less strong. In a very narrow band around $C_{\mu}^{\prime}$ equal to -1.5 , the wino is the LSP. The chargino then decays softly to a neutral wino. This is similar to gluino decaying to $\chi_{1}^{0}$ and the exclusion reaches the same levels as at $C_{\mu}^{\prime}$ equal to -3 . As $C_{\mu}^{\prime}$ continues to increase, the mass spectrum again becomes compressed to the point where gluinos can only decay to $\chi_{1}^{0}$ and a pair of soft bottom quarks and the limits drop considerably. In addition, direct electroweakino production searches from $[49-51,54]$ impose limits in a very narrow band near $C_{\mu}^{\prime}$ equal to -2 . This corresponds to when both the wino and bino are light which only occurs around $C_{\mu}^{\prime}$ equal to -2 . This region is shown as a grey band in figure 4.4. 
(a) $M_{\tilde{B}}[\mathrm{GeV}]$

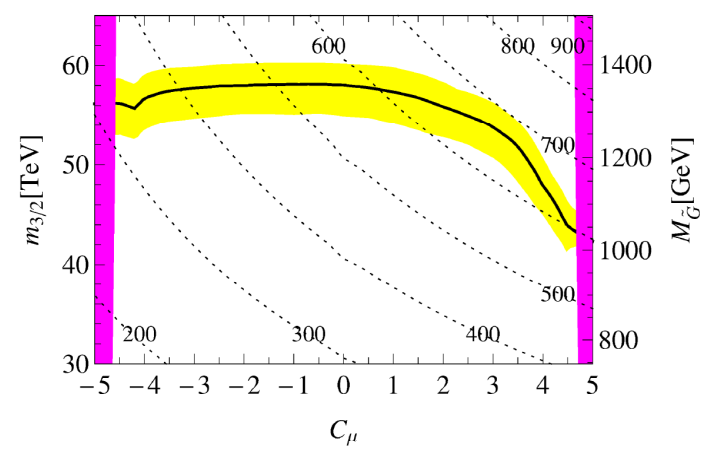

(c) $\mu[\mathrm{TeV}]$

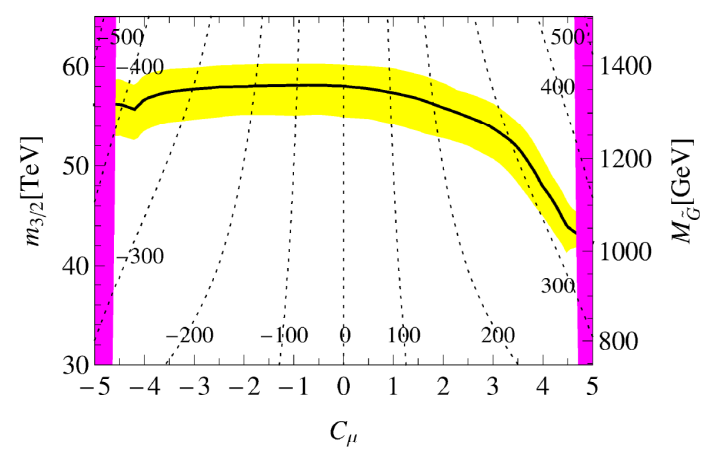

(b) $M_{\tilde{W}}[\mathrm{GeV}]$

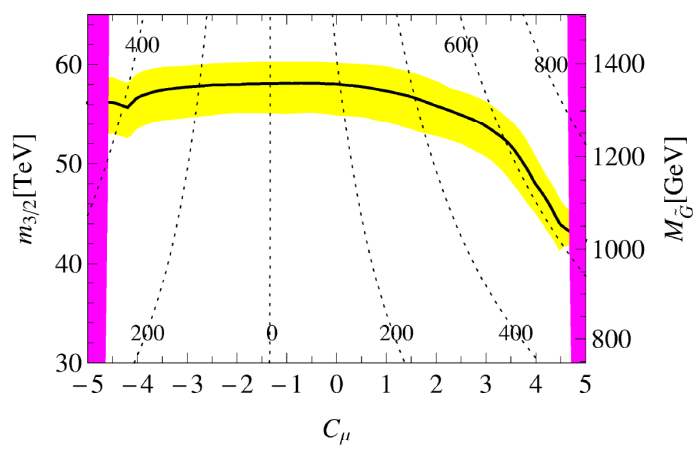

(d) $\tan \beta$

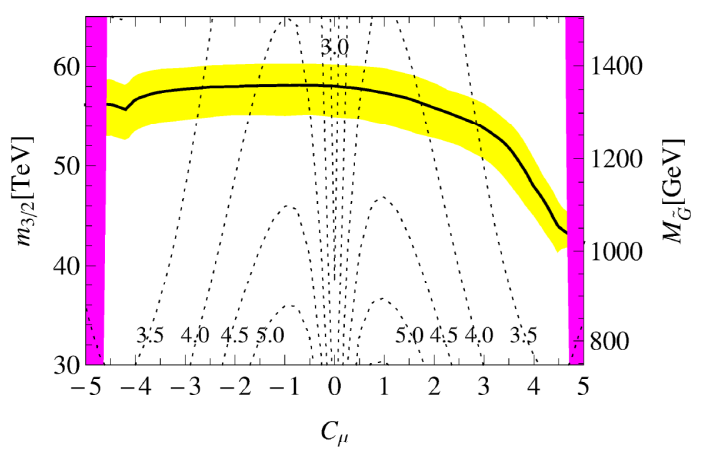

Figure 4.3: $95 \%$ exclusion limits for anomaly mediation at the LHC. The yellow band corresponds to the $1 \sigma$ uncertainty on the gluino pair production cross section and the purple bands are the forbidden region of colour-breaking vacuum. Contour lines of constant $M_{\tilde{B}}, M_{\tilde{W}}, \mu$, and $\tan \beta$ are shown respectively in (a), (b), (c), and (d). 
(a) $M_{\tilde{B}}[\mathrm{GeV}]$

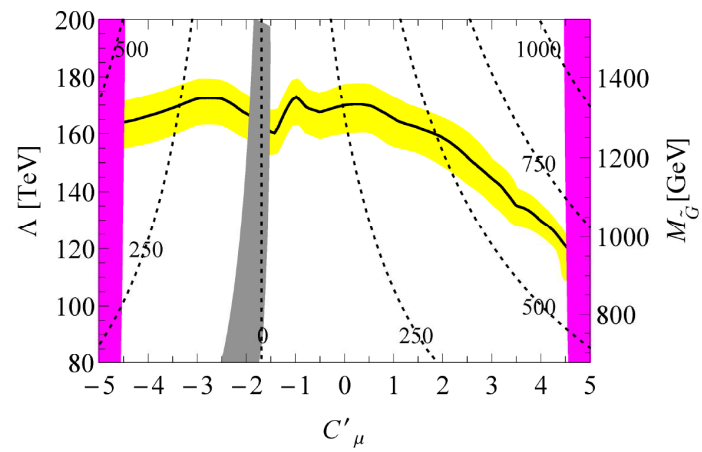

(c) $\mu[\mathrm{TeV}]$

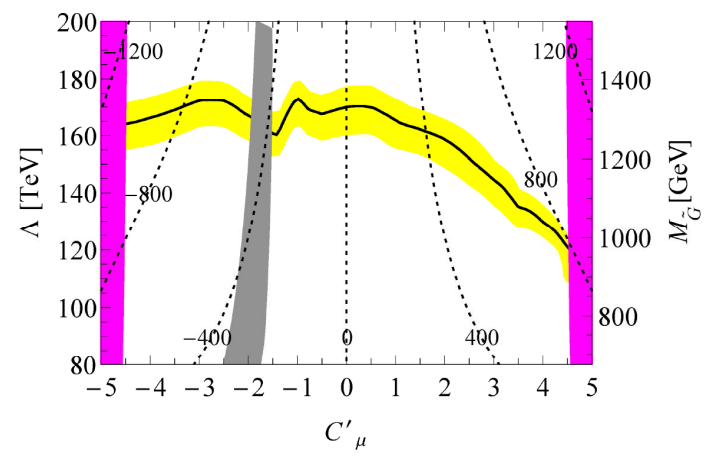

(b) $M_{\tilde{W}}[\mathrm{GeV}]$

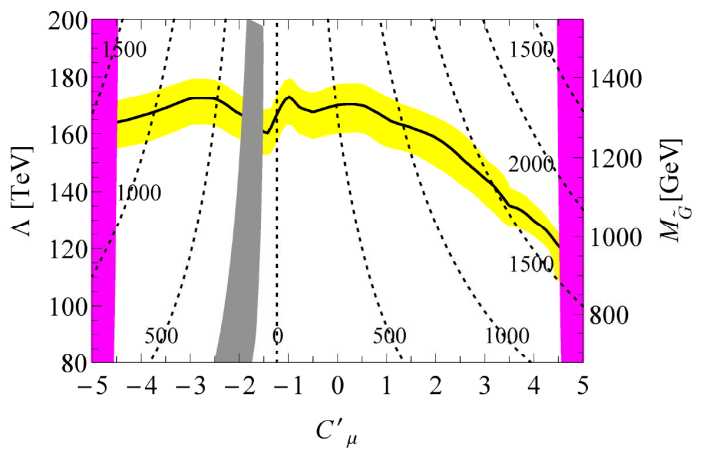

(d) $\tan \beta$

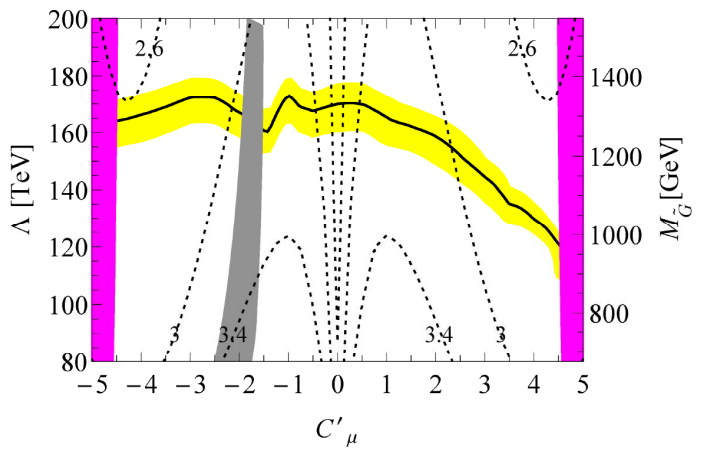

Figure 4.4: $95 \%$ exclusion limits for gauge mediation at the LHC. The yellow band corresponds to the $1 \sigma$ uncertainty on the gluino pair production cross section and the purple bands are the forbidden region of colour-breaking vacuum. The grey band corresponds to limits from direct electroweak searches. Contour lines of constant $M_{\tilde{B}}$, $M_{\tilde{W}}, \mu$, and $\tan \beta$ are shown respectively in (a), (b), (c), and (d). 


\subsubsection{Prospects at LHC 14}

The procedure of the previous section can be modified to predict the discovery and exclusion prospects at the next phase of the LHC. The only differences amount to the signal regions and background estimations.

Two different strategies are adopted to cover the possibilities of the spectrum being compressed or not. When the LSP is considerably lighter than the gluino, kinematic quantities like MET are large and strong kinematic cuts are sufficient to eliminate most of the background. We refer to these signal regions as high MET cuts. On the contrary, when the gluino has a mass close to the LSP, quantities like MET become small and the cuts remove most signals. Lowering the cuts does not improve the limits much as the background increases considerably. However, adding the requirement of same sign dileptons (SSDL) drastically cuts the background and allows the kinematic cuts to be made less stringent by exploiting the possible production of leptons during the top decay. The only drawback to SSDL is that a large part of the signal is cut and the resulting limits are less strong than pure high MET cuts in the non-compressed case. The net result is that high MET signal regions usually dominate until the spectrum becomes near degenerate. The exclusion then drops until the signal regions with SSDL become relevant which prevents the exclusion limits from dropping too fast. However, the SSDL cuts eventually also fail when there is not enough phase space for the gluino to produce top quarks.

For the high MET signal regions, we adopt the cuts of [88] for gluinos decaying to top quarks and a single lepton. The cuts for SSDL are taken directly from [89] and correspond to their gluino-neutralino model with heavy flavour decay for $14 \mathrm{TeV}$. We verified that we could reproduce both sets of results.

The detector card for Delphes is the standard $14 \mathrm{TeV}$ card from Snowmass [90]. 
The background estimates for the high MET regions are obtained from the Snowmass online backgrounds [91]. We simply apply our cuts on their events while taking into consideration their relative weight. The Snowmass backgrounds also provide event files with different average number of pile-up. In general, pile-up has very little effect on the high MET regions, while, for SSDL, leptons can possibly get lost in the pile-up jets [89], reducing the efficiency of the signal. We however concentrate on the case of 0 pile-up as the effect is generally small on most of the parameter space. For high MET cuts, we obtain backgrounds of $(23.0,12.1,2.6,2.1)$ for the four signal regions of [88] and $3000 \mathrm{fb}^{-1}$ of integrated luminosity. This can be compared with their result at 140 pile-up of $(17.5,4.8,0.9,1.6)$ and the same integrated luminosity. The backgrounds for SSDL are taken directly from [89], as we follow very closely their procedure. A $20 \%$ systematic uncertainty on all backgrounds is assumed [89]. The gluino pair production cross section is calculated using NLL-fast [79-83] customized for a $14 \mathrm{TeV}$ collider. The possibility of 300 and $3000 \mathrm{fb}^{-1}$ of integrated luminosity are considered.

The results can be seen for anomaly mediation in figures 4.5 and 4.6 for $95 \%$ exclusion and $5 \sigma$ discovery respectively, as well as for gauge mediation in figure 4.7 and 4.8 for $95 \%$ exclusion and $5 \sigma$ discovery respectively. The curves are essentially scaled up versions of the $8 \mathrm{TeV}$ constraints. The anomaly mediation limit curves are flatter than those for the current LHC constraints. This can be explained by the fact that the branching ratio to the LSP and two tops decreases more slowly as $C_{\mu}$ increases because heavier gluinos are being probed. 
(a) $M_{\tilde{B}}[\mathrm{TeV}]$

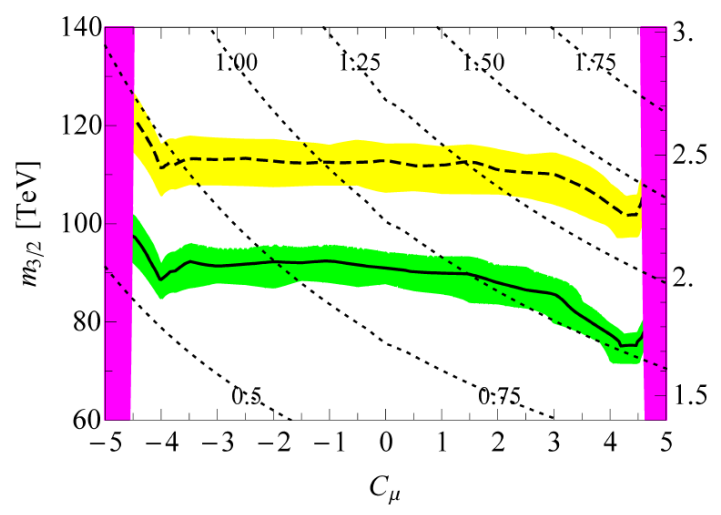

(c) $\mu[\mathrm{PeV}]$

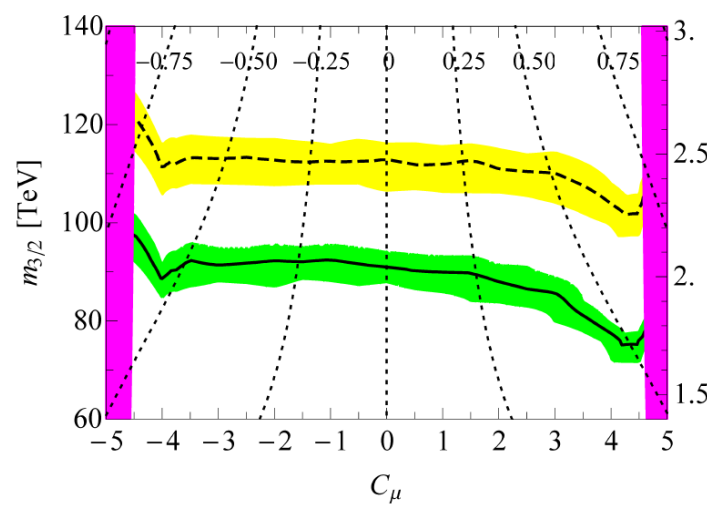

(b) $M_{\tilde{W}}[\mathrm{TeV}]$

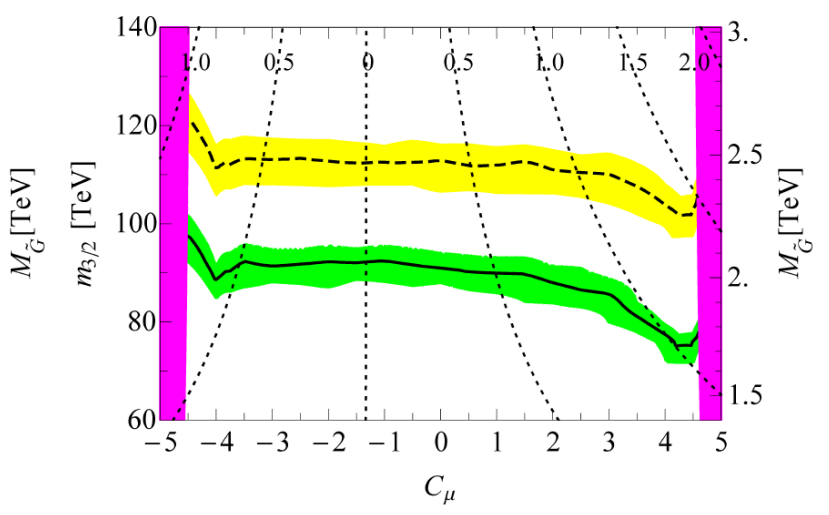

(d) $\tan \beta$

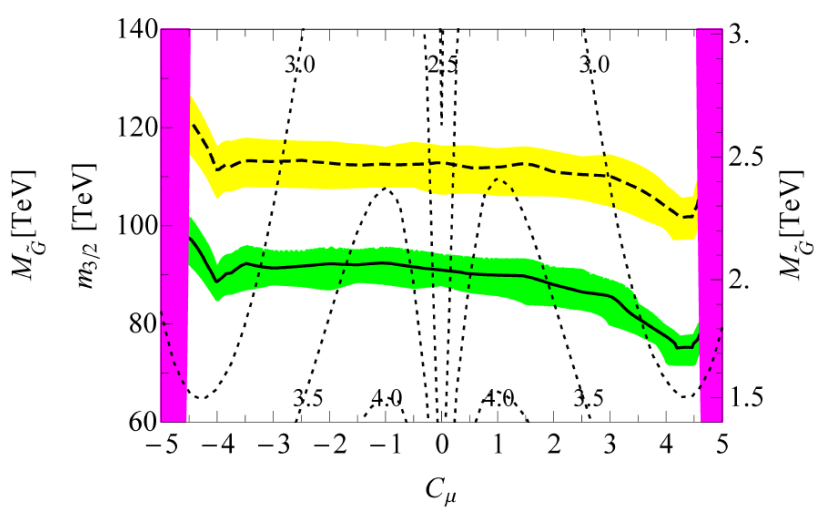

Figure 4.5: 95\% exclusion limits for anomaly mediation at LHC 14 for (solid) 300 $\mathrm{fb}^{-1}$ and (dashed) $3000 \mathrm{fb}^{-1}$ integrated luminosity. The green band corresponds to the $1 \sigma$ uncertainty on the gluino pair production cross section for $300 \mathrm{fb}^{-1}$, the yellow band corresponds to the $1 \sigma$ uncertainty on the gluino pair production cross section for $3000 \mathrm{fb}^{-1}$, and the purple bands are the forbidden region of colour-breaking vacuum. Contour lines of constant $M_{\tilde{B}}, M_{\tilde{W}}, \mu$, and $\tan \beta$ are shown respectively in (a), (b), (c), and (d). 
(a) $M_{\tilde{B}}[\mathrm{TeV}]$

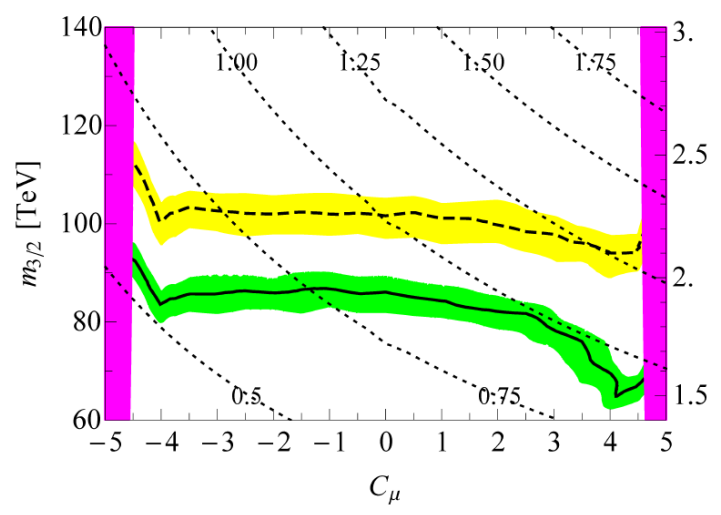

(c) $\mu[\mathrm{PeV}]$

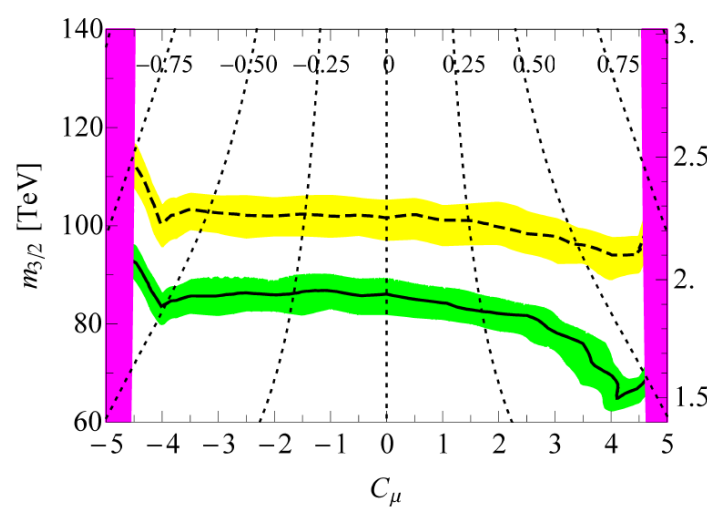

(b) $M_{\tilde{W}}[\mathrm{TeV}]$

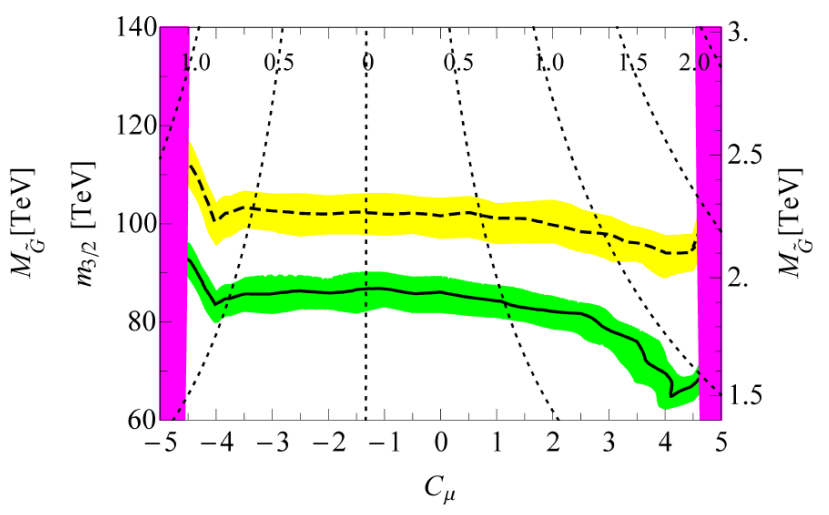

(d) $\tan \beta$

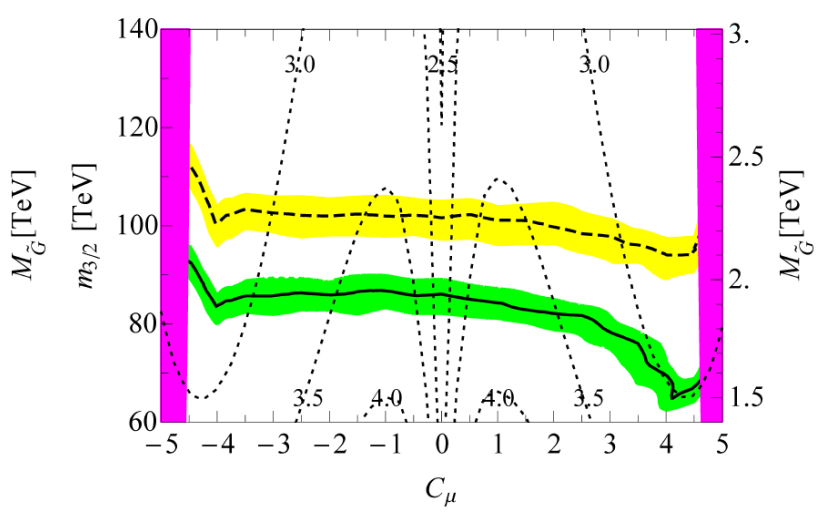

Figure 4.6: $5 \sigma$ discovery limits for anomaly mediation at LHC 14 for (solid) 300 $\mathrm{fb}^{-1}$ and (dashed) $3000 \mathrm{fb}^{-1}$ integrated luminosity. The green band corresponds to the $1 \sigma$ uncertainty on the gluino pair production cross section for $300 \mathrm{fb}^{-1}$, the yellow band corresponds to the $1 \sigma$ uncertainty on the gluino pair production cross section for $3000 \mathrm{fb}^{-1}$, and the purple bands are the forbidden region of colour-breaking vacuum. Contour lines of constant $M_{\tilde{B}}, M_{\tilde{W}}, \mu$, and $\tan \beta$ are shown respectively in (a), (b), (c), and (d). 
(a) $M_{\tilde{B}}[\mathrm{TeV}]$

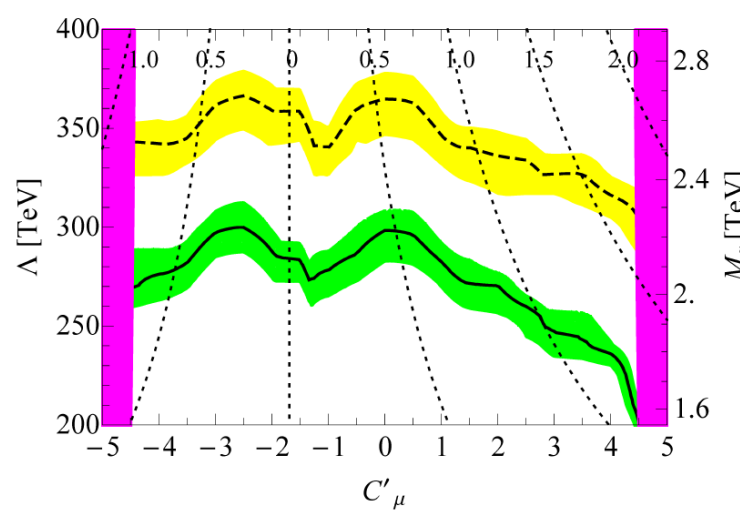

(c) $\mu[\mathrm{PeV}]$

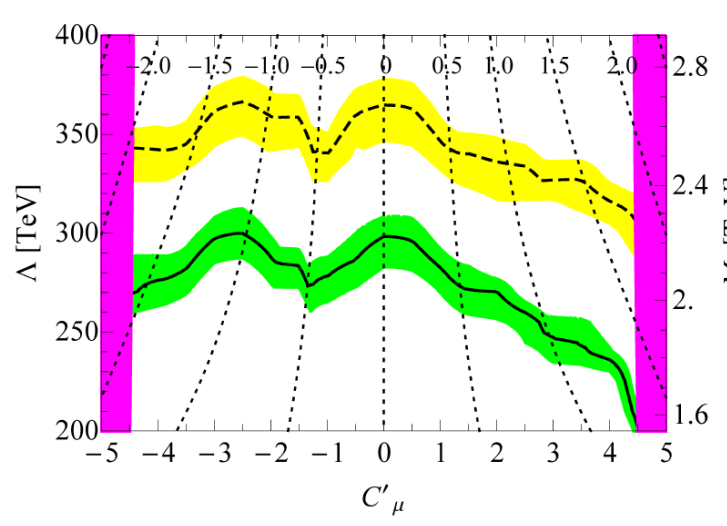

(b) $M_{\tilde{W}}[\mathrm{TeV}]$

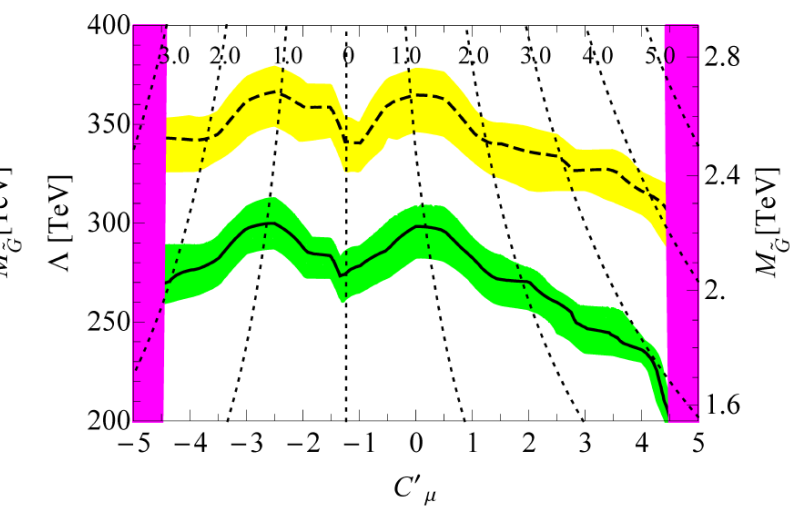

(d) $\tan \beta$

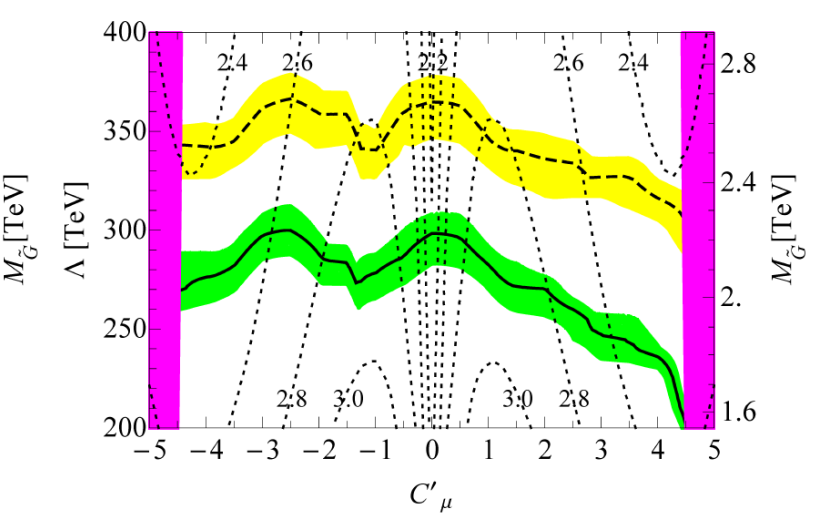

Figure 4.7: 95\% exclusion limits for gauge mediation at LHC 14 for (solid) $300 \mathrm{fb}^{-1}$ and (dashed) $3000 \mathrm{fb}^{-1}$ integrated luminosity. The green band corresponds to the $1 \sigma$ uncertainty on the gluino pair production cross section for $300 \mathrm{fb}^{-1}$, the yellow band corresponds to the $1 \sigma$ uncertainty on the gluino pair production cross section for $3000 \mathrm{fb}^{-1}$, and the purple bands are the forbidden region of colour-breaking vacuum. Contour lines of constant $M_{\tilde{B}}, M_{\tilde{W}}, \mu$, and $\tan \beta$ are shown respectively in (a), (b), (c), and (d). 
(a) $M_{\tilde{B}}[\mathrm{TeV}]$

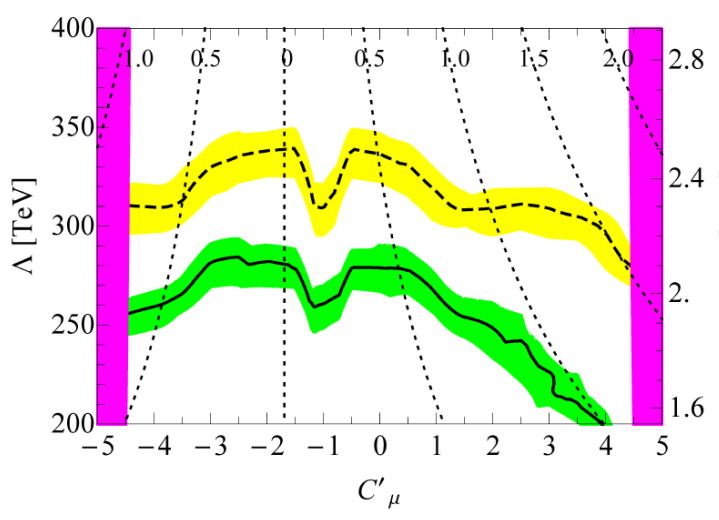

(c) $\mu[\mathrm{PeV}]$

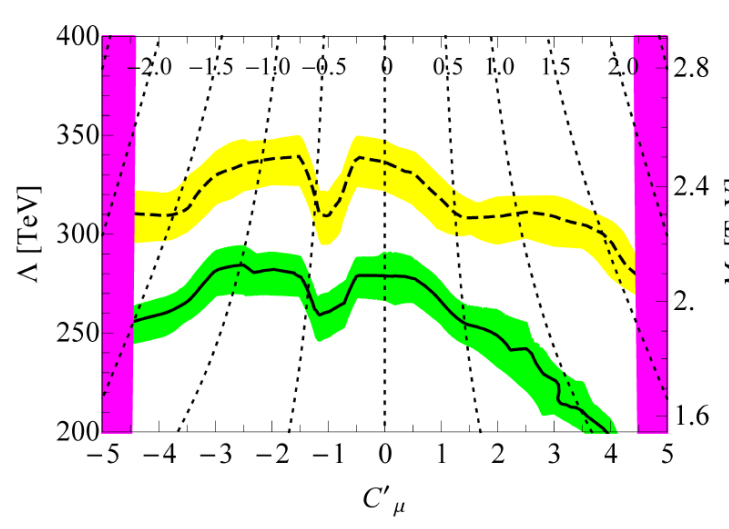

(b) $M_{\tilde{W}}[\mathrm{TeV}]$

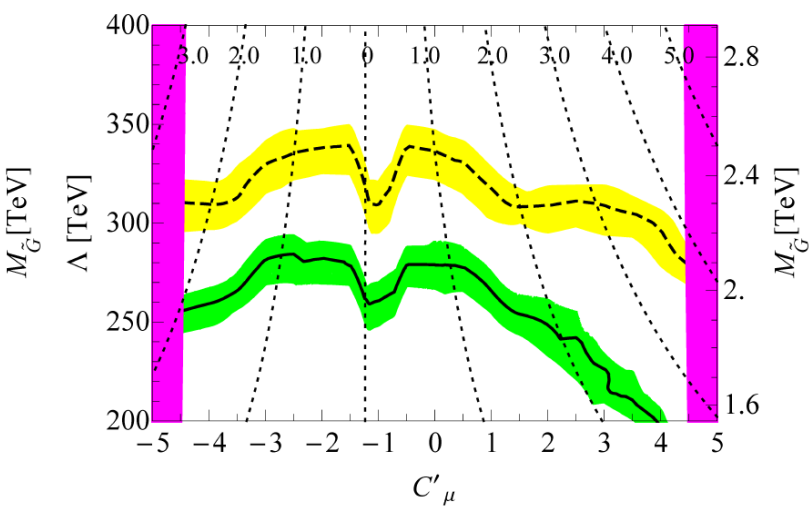

(d) $\tan \beta$

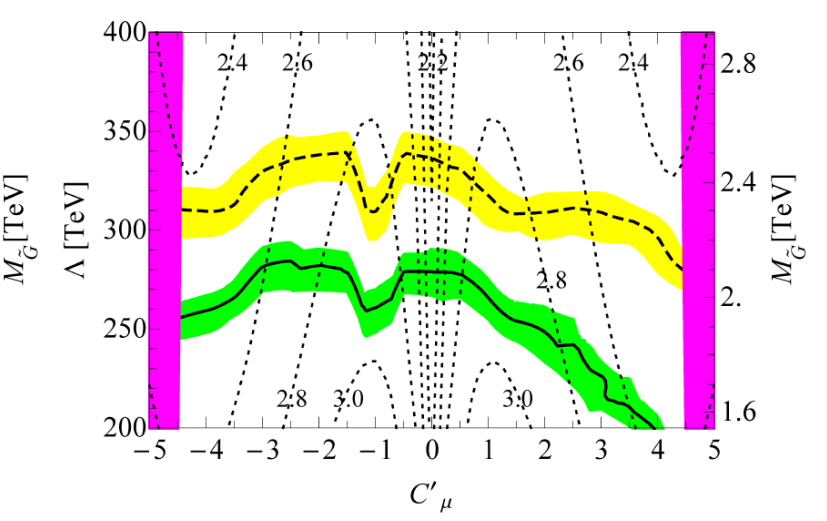

Figure 4.8: $5 \sigma$ discovery limits for gauge mediation at LHC 14 for (solid) $300 \mathrm{fb}^{-1}$ and (dashed) $3000 \mathrm{fb}^{-1}$ integrated luminosity. The green band corresponds to the $1 \sigma$ uncertainty on the gluino pair production cross section for $300 \mathrm{fb}^{-1}$, the yellow band corresponds to the $1 \sigma$ uncertainty on the gluino pair production cross section for $3000 \mathrm{fb}^{-1}$, and the purple bands are the forbidden region of colour-breaking vacuum. Contour lines of constant $M_{\tilde{B}}, M_{\tilde{W}}, \mu$, and $\tan \beta$ are shown respectively in (a), (b), (c), and (d). 


\subsubsection{Prospects at a $100 \mathrm{TeV}$ collider}

To fully explore the possibility of discovering Mini-Split supersymmetry at colliders, we study the prospects of a $100 \mathrm{TeV}$ proton proton collider following the same procedure as in the previous two sections. Our high MET cuts are adapted from [37], which are themselves based on [92]. These cuts rely on $M_{\text {eff }}$ which is defined as

$$
M_{\mathrm{eff}}=\sum_{i} p_{T}(i)+\mathrm{MET} .
$$

The sum is on jets with $p_{T}>50 \mathrm{GeV}$ and $|\eta|<5$ and leptons with $p_{T}>15 \mathrm{GeV}$ and $|\eta|<2.5$. We push things further than [37] by requiring b-jets, implementing detector simulations, and using a set of signal regions optimized for different regions of parameter space. The preselection cuts are given by [37]

- lepton veto,

- at least two jets with $p_{T}>0.1 M_{\text {eff }}$,

- $\mathrm{MET}>0.2 M_{\mathrm{eff}}$,

- $p_{T}\left(j_{1}\right)<0.35 M_{\text {eff }}$,

- $\Delta \phi\left(j_{1}, \mathrm{MET}\right)<\pi-0.2$,

- $\Delta \phi\left(j_{1}, j_{2}\right)<2 \pi / 3$.

The different signal regions correspond to different combinations of minimum b-jets requirements and $M_{\text {eff }}$ cuts and are given in table 4.2.

The SSDL cuts and the corresponding backgrounds are taken directly from [89] and correspond to their search for a gluino-neutralino model with heavy flavour decays. We verified that we could reproduce their results. 


\begin{tabular}{|c|c|c|c|}
\hline SR & b-jets & $M_{\text {eff }}[\mathrm{TeV}]$ & Background \\
\hline hMETb3A & $\geq 3$ & $>15.0$ & 23.4 \\
\hline hMETb3B & $\geq 3$ & $>17.5$ & 7.8 \\
\hline hMETb3C & $\geq 3$ & $>20.0$ & 2.3 \\
\hline hMETb4A & $\geq 4$ & $>12.5$ & 12.6 \\
\hline hMETb4B & $\geq 4$ & $>15.0$ & 3.8 \\
\hline hMETb4C & $\geq 4$ & $>17.5$ & 1.5 \\
\hline hMETb4D & $\geq 4$ & $>20.0$ & 0.5 \\
\hline
\end{tabular}

Table 4.2: Signal regions for high MET. The background for $3 \mathrm{ab}^{-1}$ is also included.

The detector card for Delphes is the standard 100 TeV card from Snowmass [90]. The background estimates for high MET are again obtained from the Snowmass online backgrounds [91]. The backgrounds for the high MET signal regions are shown in table 4.2 for $3 \mathrm{ab}^{-1}$ integrated luminosity. A $20 \%$ systematic uncertainty on all backgrounds is assumed [89]. The discussion of pile-up for high MET or SSDL from the previous section still holds. We concentrate on the 0 pile-up case, as the average pile-up of a future $100 \mathrm{TeV}$ collider is still unknown and as it only has a non-negligible effect on a small portion of our parameter space. The gluino pair production cross section is calculated using NLL-fast [79-83] customized for a $100 \mathrm{TeV}$ collider.

The results are again scaled up versions of LHC constraints with possible exclusion of up to a $14 \mathrm{TeV}$ gluino in a large region of parameter space and discovery of up to $12 \mathrm{TeV}$. These numbers are similar to those obtained by [37] which seem somewhat more optimistic (with a possible discovery of up to $\sim 15 \mathrm{TeV}$ ). ${ }^{4}$ For anomaly mediation, exclusion limits are governed by high MET signal regions and are thus very high until $C_{\mu}$ reaches 1. At this point, the spectrum becomes compressed and the limits drop. The SSDL bins then dominate and the limits stabilize with a discovery reach of about $7 \mathrm{TeV}$ (this number is in fact quite close to the result of [89]). The exact

\footnotetext{
${ }^{4}$ This might be due, for example, to the fact that we have used a detector simulation, but we have not directly checked that hypothesis.
} 
(a) $M_{\tilde{B}}[\mathrm{TeV}]$

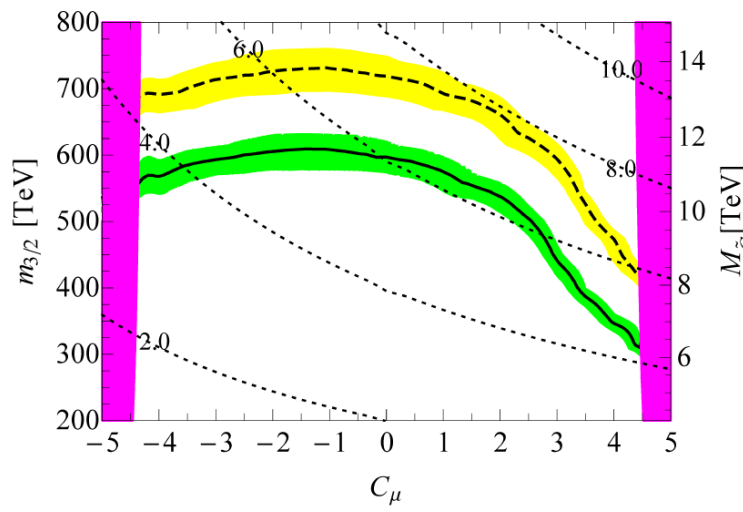

(c) $\mu[\mathrm{PeV}]$

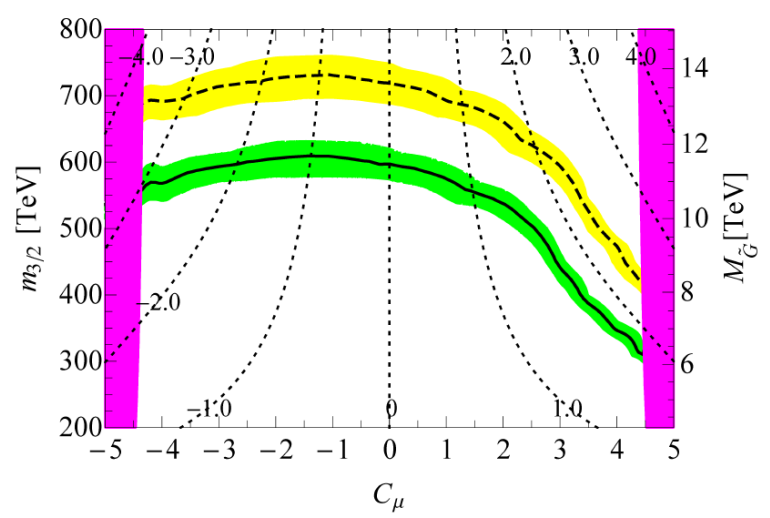

(b) $M_{\tilde{W}}[\mathrm{TeV}]$

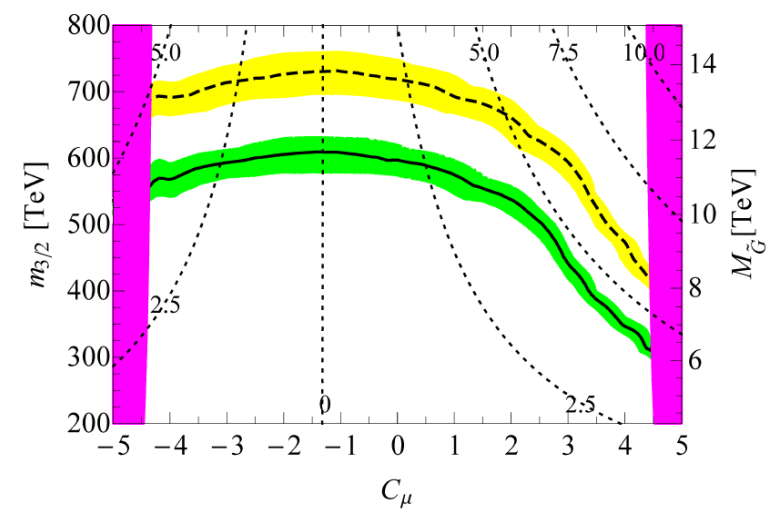

(d) $\tan \beta$

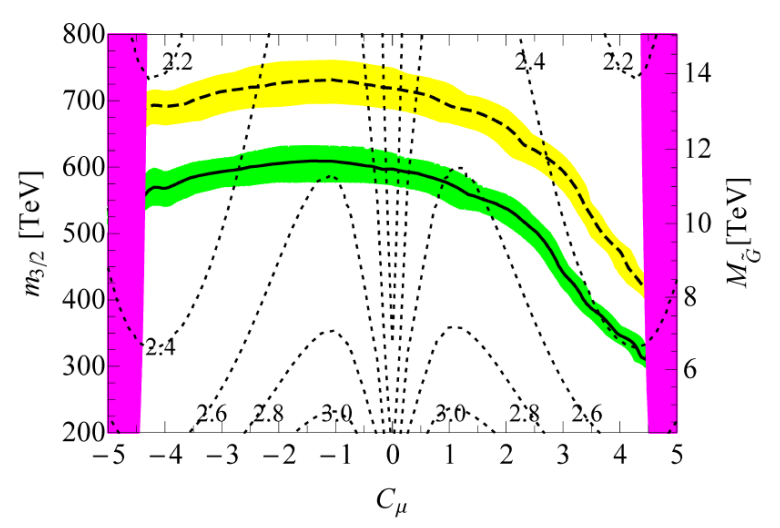

Figure 4.9: $95 \%$ (dashed) exclusion and $5 \sigma$ (solid) discovery limits for anomaly mediation at a $100 \mathrm{TeV} p p$ collider with $3 \mathrm{ab}^{-1}$ integrated luminosity. The yellow band corresponds to the $1 \sigma$ uncertainty on the gluino pair production cross section for $95 \%$ exclusion, the green band corresponds to the $1 \sigma$ uncertainty on the gluino pair production cross section for $5 \sigma$ discovery, and the purple bands are the forbidden region of colour-breaking vacuum. Contour lines of constant $M_{\tilde{B}}, M_{\tilde{W}}, \mu$, and $\tan \beta$ are shown respectively in (a), (b), (c), and (d). 
(a) $M_{\tilde{B}}[\mathrm{TeV}]$

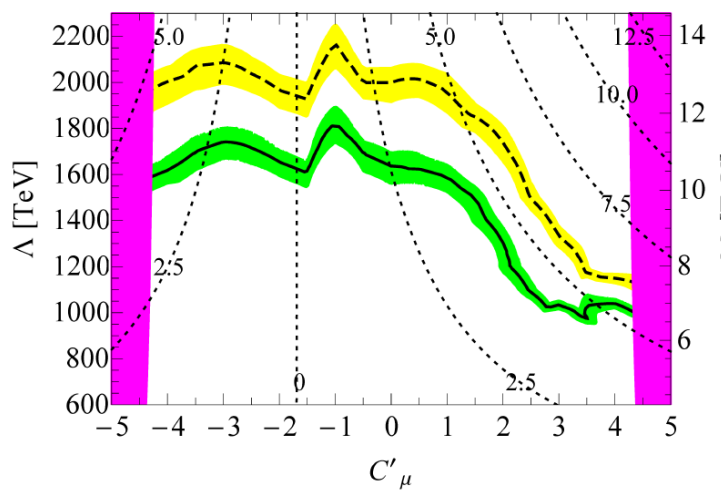

(c) $\mu[\mathrm{PeV}]$

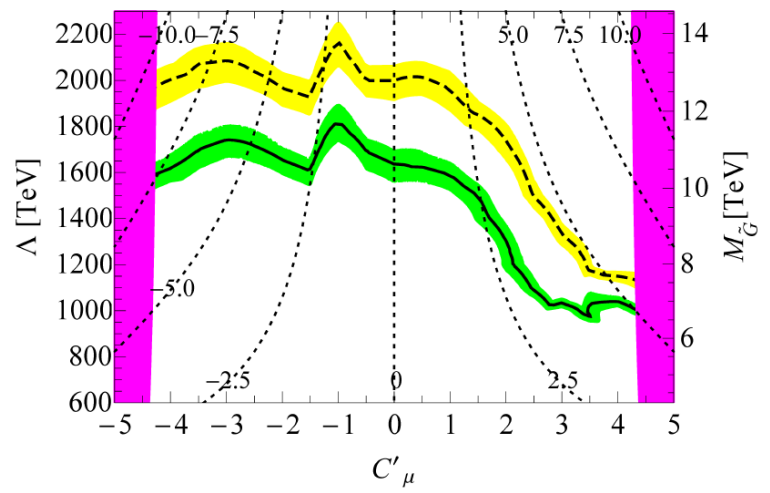

(b) $M_{\tilde{W}}[\mathrm{TeV}]$

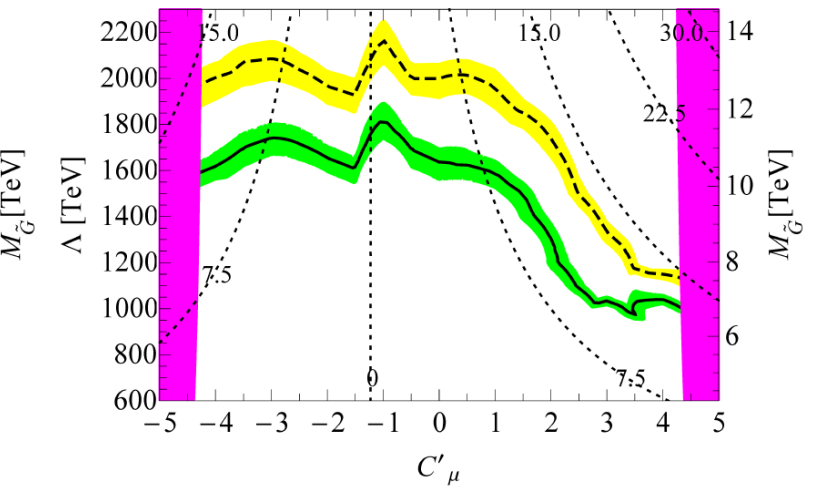

(d) $\tan \beta$

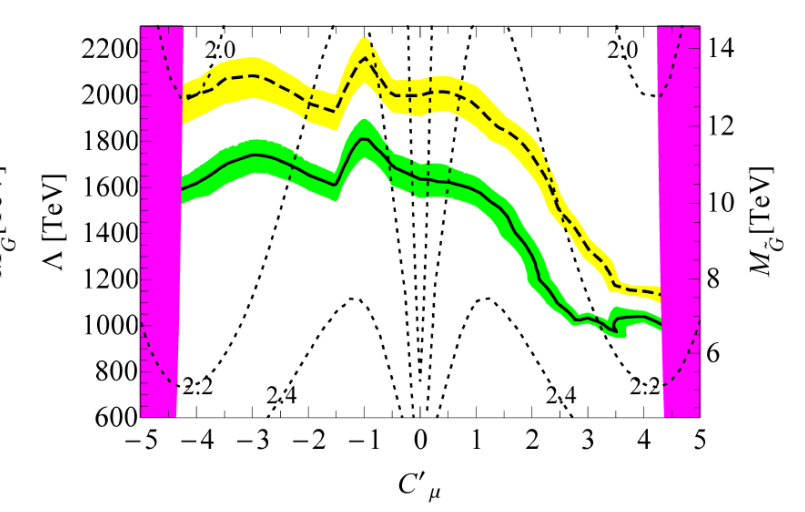

Figure 4.10: $95 \%$ (dashed) exclusion and $5 \sigma$ (solid) discovery limits for gauge mediation at a $100 \mathrm{TeV} p p$ collider with $3 \mathrm{ab}^{-1}$ integrated luminosity. The yellow band corresponds to the $1 \sigma$ uncertainty on the gluino pair production cross section for $95 \%$ exclusion, the green band corresponds to the $1 \sigma$ uncertainty on the gluino pair production cross section for $5 \sigma$ discovery, and the purple bands are the forbidden region of colour-breaking vacuum. Contour lines of constant $M_{\tilde{B}}, M_{\tilde{W}}, \mu$, and $\tan \beta$ are shown respectively in (a), (b), (c), and (d). 
same thing happens in the case of gauge mediation, except that the limits drop at $C_{\mu}^{\prime}$ equal to 0 .

\subsection{Conclusion}

In light of ever stronger constraints from collider physics, Mini-Split scenarios become more and more appealing. In these models, a small hierarchy exists between the sfermions and gauginos, with the gauginos being near the electroweak scale. This kind of spectrum could easily arise from anomaly mediation and from gauge mediation. In these models the electroweak scale is tuned, but the gauge couplings could still unify at a high scale, and the models have possible dark matter candidates. The hierarchy between the scalars and the gauginos leads to large radiative corrections which can greatly modify the standard mass spectra of anomaly and gauge mediation.

In this chapter we studied hadron collider constraints and prospects on these deflected anomaly mediation and deflected gauge mediation models. By using a simple parametrization of the models and assuming a lighter third generation and a heavy Higgsino, we recast SUSY searches from ATLAS and CMS to obtain exclusions on the parameter space of the models. The known mass of the Higgs boson and the absence of a colour-breaking vacuum were also taken into account. Results for anomaly and gauge mediation can be seen respectively in figures 4.3 and 4.4. We also obtained future prospects for deflected anomaly mediation and deflected gauge mediation for LHC 14 and a $100 \mathrm{TeV}$ proton proton collider. For LHC 14, the 95\% projected exclusion limits are shown in figures 4.5 and 4.7 for anomaly and gauge mediation respectively and the $5 \sigma$ discovery prospects are shown in figures 4.6 and 4.8. The prospects at a $100 \mathrm{TeV}$ collider for anomaly and gauge mediation are found in figures 4.9 and 4.10 respectively. 
While the goal of this chapter was to explore the collider phenomenology of Mini-Split models, dark matter properties could also be used to further restrict the parameter space. The thermal abundance of the dark matter candidate is strongly dependent on the identity of the LSP. For a Wino LSP, the correct thermal relic abundance can be obtained for a wino mass of $2.7 \mathrm{TeV}[36,57]$. This region of parameter space is not constrained by the LHC, but is within reach of a $100 \mathrm{TeV}$ collider. Wino LSP with lighter mass could be accommodated by invoking non-thermal production [57]. Similarly, Bino LSP, which tend to overclose the universe, could be accommodated if there was late entropy production or a low reheating temperature. 


\section{Chapter 5}

\section{Phenomenology of Higgsinos with}

\section{baryonic $R$-parity violation at the}

\section{LHC}

\section{$5.1 \quad$ Introduction}

In natural supersymmetric theories, the Higgsino mass parameter $\mu$ is typically of the order of the electroweak scale. This is required so that the $Z$ mass determined by the equation

$$
m_{Z}^{2}=\frac{m_{H_{u}}^{2}-m_{H_{d}}^{2}}{\cos (2 \beta)}-m_{H_{u}}^{2}-m_{H_{d}}^{2}-2 \mu^{2}
$$

is not significantly fine-tuned. If this is the case, then there should be at least two neutralinos and one chargino with masses of a few hundred GeV. Moreover, if the bino and wino soft supersymmetry breaking mass terms are larger than $\mu$, then these light neutralinos and chargino will be almost purely Higgsinos and their masses will 
be nearly degenerate.

The ATLAS and CMS collaborations at the LHC have preformed exhaustive searches looking for supersymmetric signatures. Depending on the exact details of the simplified model under consideration, many of these searches constrain the masses of neutralinos and charginos. For example, if $R$-parity is conserved, then the lightest neutralino is stable (assuming it is the lightest supersymmetric particle). Constraints can be derived from strongly produced supersymmetric particles which decay to SM particles and a pair of lightest neutralinos [93-96]. Additionally, neutralinos and charginos can also potentially be directly pair produced through electroweak processes resulting in bounds on their masses [97-99] provided there is a mass splitting between the lightest neutralino and the second lightest neutralino or chargino. On the other hand, if $R$-parity is violated, then additional decay possibilities open as the lightest neutralino is no longer stable. If the $R$-parity violating couplings are small, then production of neutralinos can lead to displaced vertices [100,101]. If the $R$-parity violating couplings are large, then the neutralinos and charginos decay promptly to final states featuring only SM particles. This affects the phenomenology of both strongly produced particles $[102,103]$ and electroweak production of neutralinos and charginos $[104,105]$.

There have also been several phenomenological studies examining many kinds of $R$-parity violating supersymmetric models at the LHC [106-135]. These studies have considered both strong and electroweak production of supersymmetric particles and have analyzed the subsequent large variety of possible decay chains resulting in final states with only SM particles due to the $R$-parity violating couplings. Again, if the $R$-parity violating couplings are small then bounds can be derived from displaced vertices whereas if they are large, then the decay chains occur promptly. 
One potentially interesting phenomenological signature that has not been examined is the pair production of nearly degenerate Higgsino neutralinos and charginos at the LHC which then promptly decay due to the $\lambda^{\prime \prime} U^{c} D^{c} D^{c}$ superpotential operator. In fact, this is in stark contrast to the operators $\lambda L L E^{c}$ and $\lambda^{\prime} L Q D^{c}$. For both of these operators, the CMS search [104] has explicitly considered the pair production of Higgsinos which then promptly decay due to the $\lambda$ and $\lambda^{\prime}$ couplings. This has led to bounds on the Higgsino masses of a few hundred $\mathrm{GeV}$, dependent, of course, on the exact flavour structure of the $\lambda$ or $\lambda^{\prime}$ coupling under consideration. The difference between these operators and the $\lambda^{\prime \prime} U^{c} D^{c} D^{c}$ operator is that these operators result in the neutralinos and charginos decaying to particles of which some or all are leptons whereas the operator $\lambda^{\prime \prime} U^{c} D^{c} D^{c}$ results in the neutralinos and charginos decaying only to quarks. Indeed, it seems challenging to derive bounds from electroweakly produced particles which decay promptly to quarks at a proton proton collider. In this chapter we show that it is possible. To do so, we turn on a single coupling of the form $\lambda_{3 j k}^{\prime \prime}$. Higgsino neutralinos can then decay to a top and two down quarks. Further, provided the Higgsino neutralinos are Majorana states (instead of a single Dirac state), then pair produced Higgsinos can lead to same sign tops. Same sign tops can lead to same sign leptons and by recasting a CMS search [136] looking for this distinctive signature and multiple b-jets, we will show that parts of the parameters space are beginning to be constrained.

There are also bounds on the $\lambda^{\prime \prime}$ couplings coming from baryon number violating processes and flavour physics. A more thorough discussion of these bounds is presented in section 6.2.1. Here we simply summarize the main results. Bounds from baryon number violating processes constrain individual $\lambda_{i j k}^{\prime \prime}$ whereas bounds from flavour physics constrain combinations of $\lambda^{\prime \prime}$ couplings with different flavour struc- 
tures [19]. Examples of baryon number violating processes that lead to bounds include proton decay, neutron antineutron oscillation, and double nucleon decay $[19,137]$. Examples of flavour physics observables which lead to bounds are $\epsilon_{K}$ and $\Delta m_{K}$ [138]. All bounds can be avoided provided only one coupling of the form $\lambda_{3 j k}^{\prime \prime}$ in the mass basis is taken to be large. Whether or not it is possible to build a model of flavour where this naturally occurs remains to be seen.

This chapter is organized as follows. First, section 5.2 presents a detailed discussion of Majorana and Dirac Higgsino neutralinos. This section considers two nearly degenerate Higgsino neutralino states and determines the condition in which it is more appropriate to treat the two states as asymptotic Majorana particles or as a single Dirac particle. It is shown that this affects the production of opposite sign and same sign tops originating from decaying Higgsino neutralinos. We then present in section 5.3 the masses of the Higgsino neutralinos and chargino including tree-level mixing effects involving the winos and the bino as well as one loop contributions. The LHC phenomenology is then studied in section 5.4. This includes a discussion of how the Higgsino neutralinos and chargino decay, the procedure used to constrain individual points in parameter space, the method to scan the parameter space, and finally our results. A conclusion to this chapter is presented in section 5.5.

\subsection{Majorana versus Dirac Higgsinos}

Neutralino mass terms in the MSSM are given by [16]

$$
\mathcal{L}=-\frac{1}{2}\left(\psi^{0}\right)^{T} M_{\tilde{N}} \psi^{0}+\text { h.c. }
$$


where $\psi^{0}$ is a vector consisting of the Weyl spinors for the bino, neutral wino, and neutral Higgsinos, $\psi^{0}=\left(\tilde{B}, \tilde{W}^{0}, \tilde{H}_{d}^{0}, \tilde{H}_{u}^{0}\right)$, and the mass matrix $M_{\tilde{N}}$ is

$$
M_{\tilde{N}}=\left(\begin{array}{cccc}
M_{1} & 0 & -c_{\beta} s_{W} m_{Z} & s_{\beta} s_{W} m_{Z} \\
0 & M_{2} & c_{\beta} c_{W} m_{Z} & -s_{\beta} c_{W} m_{Z} \\
-c_{\beta} s_{W} m_{Z} & c_{\beta} c_{W} m_{Z} & 0 & -\mu \\
s_{\beta} s_{W} m_{Z} & -s_{\beta} c_{W} m_{Z} & -\mu & 0
\end{array}\right)
$$

where $s_{\beta}=\sin \beta, c_{\beta}=\cos \beta, s_{W}=\sin \theta_{W}$, and $c_{W}=\cos \theta_{W}$. Similarly, chargino mass terms are given by [16]

$$
\mathcal{L}=-\frac{1}{2}\left(\psi^{ \pm}\right)^{T} M_{\tilde{C}} \psi^{ \pm}+\text {h.c. }
$$

where $\psi^{ \pm}$is a vector consisting of the Weyl spinors for the positively and negatively charged winos and Higgsinos, $\psi^{ \pm}=\left(\tilde{W}^{+}, \tilde{H}_{u}^{+}, \tilde{W}^{-}, \tilde{H}_{d}^{-}\right)$, and the mass matrix $M_{\tilde{C}}$ is

$$
M_{\tilde{C}}=\left(\begin{array}{cc}
0 & X^{T} \\
X & 0
\end{array}\right) \quad \text { with } \quad X=\left(\begin{array}{cc}
M_{2} & \sqrt{2} s_{\beta} m_{W} \\
\sqrt{2} c_{\beta} m_{W} & \mu
\end{array}\right)
$$

In these matrices, the free parameters are $\tan \beta$, the soft supersymmetry breaking bino and wino mass terms $M_{1}$ and $M_{2}$, and the supersymmetric Higgsino mass term $\mu$. In general, $M_{1}, M_{2}$, and $\mu$ can have arbitrary complex phases. However, we can always rephase one of these masses so that it is real and positive [139]. We choose to perform this rephasing on $M_{2}$ and therefore consider $M_{2}>0$. Moreover, complex phases for $M_{1}$ and $\mu$ will lead to CP violating effects such as electric dipole moments and, as a result, are highly constrained [140-143]. Because of this, we consider $M_{1}$ and $\mu$ to be real, although the signs of these mass terms are still free parameters. 
The mass matrices 5.3 and 5.5 generically result in four Majorana neutralinos and two Dirac charginos after they are diagonalized. However, consider taking the limit in which both $\left|M_{1}\right| \gg|\mu|$ and $M_{2} \gg|\mu|$. In this limit, there is a Higgsino chargino, $\chi^{-}$, which to zeroth order is simply

$$
\chi^{-}=\left(\begin{array}{c}
\tilde{H}_{d}^{-} \\
\epsilon \tilde{H}_{u}^{+\dagger}
\end{array}\right)
$$

with mass $m_{\chi^{-}}=+\mu$. Additionally, the masses for the neutral Higgsinos become to zeroth order

$$
\mathcal{L}=-\frac{1}{2}\left(\begin{array}{cc}
\tilde{H}_{d}^{0} & \tilde{H}_{u}^{0}
\end{array}\right)\left(\begin{array}{cc}
0 & -\mu \\
-\mu & 0
\end{array}\right)\left(\begin{array}{c}
\tilde{H}_{d}^{0} \\
\tilde{H}_{u}^{0}
\end{array}\right)+\text { h.c. }
$$

Of course, a mass matrix of this form is simply a mass matrix for a Dirac fermion

$$
\chi_{D}^{0}=\left(\begin{array}{c}
\tilde{H}_{d}^{0} \\
\epsilon \tilde{H}_{u}^{0 \dagger}
\end{array}\right)
$$

with mass $m_{\chi_{D}^{0}}=-\mu$. Alternatively, the mass matrix in equation 5.7 can also be diagonalized, which results in two Majorana fermions

$$
\chi_{S}^{0}=\frac{\tilde{H}_{d}^{0}+\tilde{H}_{u}^{0}}{\sqrt{2}} \quad \text { and } \quad \chi_{A}^{0}=\frac{\tilde{H}_{d}^{0}-\tilde{H}_{u}^{0}}{\sqrt{2}}
$$

with masses $m_{\chi_{S}^{0}}=-\mu$ and $m_{\chi_{A}^{0}}=+\mu$, respectively.

We see that in this limit, there are two ways of treating the neutral Higgsinos. One way has a single Dirac particle, $\chi_{D}^{0}$, and the other has two mass degenerate (up to a sign) Majorana particles, $\chi_{S}^{0}$ and $\chi_{A}^{0}$. As expected, when higher order corrections 


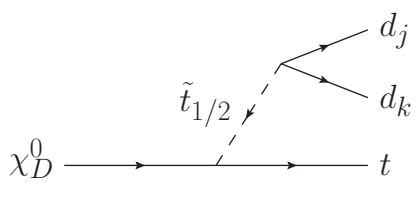

(a)

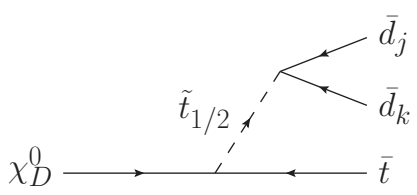

(d)

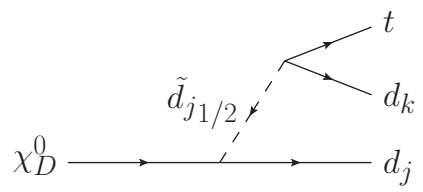

(b)

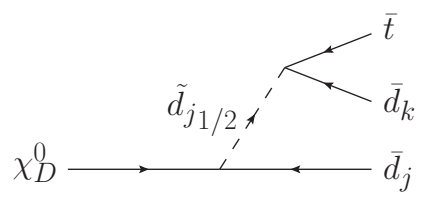

(e)

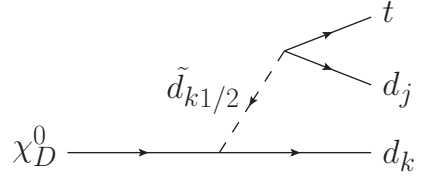

(c)

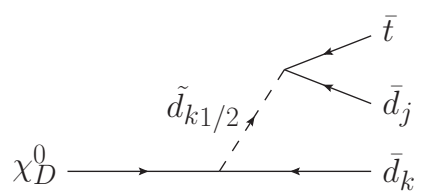

(f)

Figure 5.1: Possible decays of a Dirac Higgsino due to $\lambda_{3 j k}^{\prime \prime}$. The top row shows the diagrams for $\chi_{D}^{0} \rightarrow t d_{j} d_{k}$ and the bottom row shows the diagrams for $\chi_{D}^{0} \rightarrow \bar{t} \bar{d}_{j} \bar{d}_{k}$.

are included, the Majorana particle states will be identified as the asymptotic states. But it will prove useful to compare the possible decays and decay widths of the Dirac state $\chi_{D}^{0}$ and the two Majorana states $\chi_{S}^{0}$ and $\chi_{A}^{0}$. To allow these particles to decay, we consider the $R$-parity violating superpotential operator

$$
W \supset \frac{1}{2} \lambda_{i j k}^{\prime \prime} U_{i}^{c} D_{j}^{c} D_{k}^{c}
$$

where we have suppressed the colour indices. Gauge invariance requires these couplings to be antisymmetric with respect to the $j$ and $k$ indices, $\lambda_{i j k}^{\prime \prime}=-\lambda_{i k j}^{\prime \prime}$. As a definite example, consider the coupling $\lambda_{3 j k}^{\prime \prime}$ (with $j<k$ ). The Higgsino states can then potentially decay to final states $t d_{j} d_{k}$ and $\bar{t} \bar{d}_{j} \bar{d}_{k}$ through an internal stop or down-type squark.

In particular, consider the possible decays of the Dirac state $\chi_{D}^{0}$, which are shown in figure 5.1. The top row of this figure shows the decay $\chi_{D}^{0} \rightarrow t d_{j} d_{k}$ and the bottom row shows $\chi_{D}^{0} \rightarrow \bar{t} \bar{d}_{j} \bar{d}_{k}$. In the limit of zero left-right squark mixing, the decay $\chi_{D}^{0} \rightarrow t d_{j} d_{k}$ only proceeds through a (right-handed) stop and the decay $\chi_{D}^{0} \rightarrow \bar{t} \bar{d}_{j} \bar{d}_{k}$ 
only proceeds through (right-handed) down-type squarks, figures 5.1a, 5.1e, and 5.1f. Turning on left-right squark mixing results in the other figures, 5.1b, 5.1c, and 5.1d, having non-zero amplitudes. Therefore, both types of decays have typically non-zero, but unrelated, amplitudes.

These decays can be contrasted with the decays of the Majorana states, $\chi_{S(A)}^{0} \rightarrow$ $t d_{j} d_{k}$ and $\chi_{S(A)}^{0} \rightarrow \bar{t}_{j} \bar{d}_{k}$. The diagrams for these decays are the same as in figure 5.1 except with $\chi_{D}^{0}$ replaced with $\chi_{S(A)}^{0}$ and the arrow on the decaying Higgsino line removed. Because $\chi_{S}^{0}$ and $\chi_{A}^{0}$ are Majorana fermions, they decay equally into $t d_{j} d_{k}$ and $\bar{t} \bar{d}_{j} \bar{d}_{k}$, so that their branching ratios into these two final states are both $1 / 2$. However, the total widths of $\chi_{S}^{0}$ and $\chi_{A}^{0}$ are generally not equal, with one width larger and the other width smaller than the total width of $\chi_{D}^{0}$. In fact, the widths of the Dirac and Majorana states satisfy the relation

$$
\Gamma_{\chi_{D}^{0}}^{\text {total }}=\frac{1}{2}\left(\Gamma_{\chi_{S}^{0}}^{\text {total }}+\Gamma_{\chi_{A}^{0}}^{\text {total }}\right)
$$

As an example, consider turning on the coupling $\lambda_{312}^{\prime \prime}$. We choose $\lambda_{312}^{\prime \prime}$ so that we can neglect the diagrams with the down-type squarks due to the small down and strange Yukawa couplings. In figure 5.2a, we show the decay width for $\chi_{D}^{0} \rightarrow t d s$ and $\chi_{D}^{0} \rightarrow \bar{t} \bar{d} \bar{s}$, as well as the total decay width for $\chi_{D}^{0}$, as a function of the stop mixing angle, $\theta_{\tilde{t}}$. In this figure, and also for the other three shown in figure 5.2 , we have set $\lambda_{312}^{\prime \prime}=1, \tan \beta=40, \mu=500 \mathrm{GeV}, m_{\tilde{t}_{1}}=800 \mathrm{GeV}$, and $m_{\tilde{t}_{2}}=1400 \mathrm{GeV}$. The stop mixing angle $\theta_{\tilde{t}}$ is defined by the relations $\tilde{t}_{1}=\cos \theta_{\tilde{t}} \tilde{t}_{L}+\sin \theta_{\tilde{t}} \tilde{t}_{R}$ and $\tilde{t}_{2}=-\sin \theta_{\tilde{t}} \tilde{t}_{L}+\cos \theta_{\tilde{t}} \tilde{t}_{R}$, with $m_{\tilde{t}_{1}}<m_{\tilde{t}_{2}}$. As expected, $\chi_{D}^{0}$ decays only to the final state $t d s$ when there is zero mixing between the left and right-handed stops. Also, the decay width into $\bar{t} \bar{d} \bar{s}$ is largest when the stops are maximally mixed, $\theta_{\tilde{t}}=-\pi / 4$ or $\pi / 4$.

In figure $5.2 \mathrm{~b}$, we show the decay widths for $\chi_{S}^{0}$ and $\chi_{A}^{0}$ as a function of the 


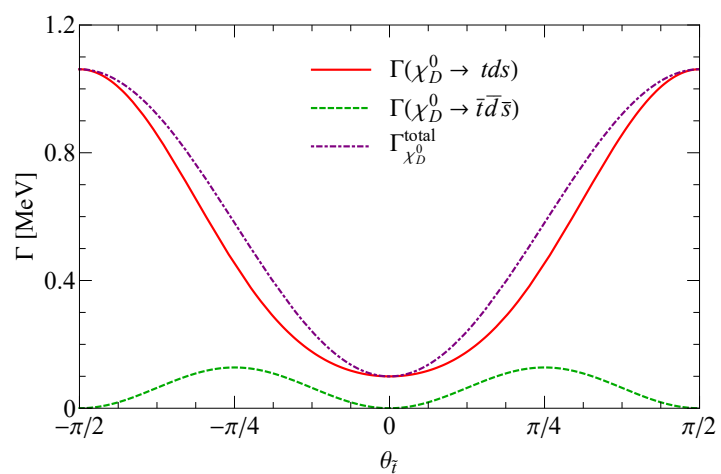

(a)

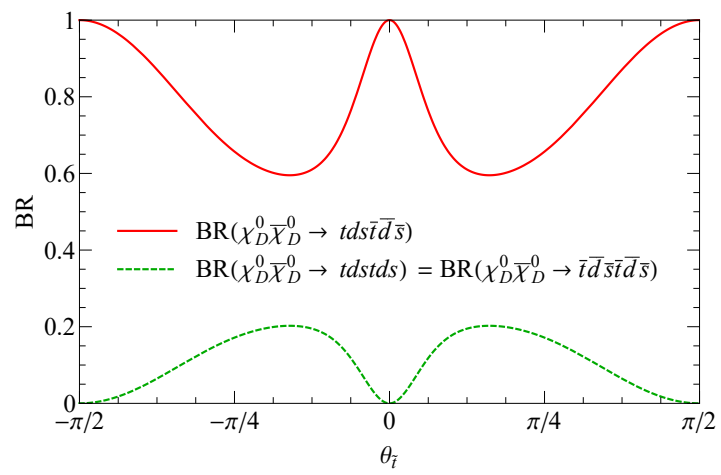

(c)

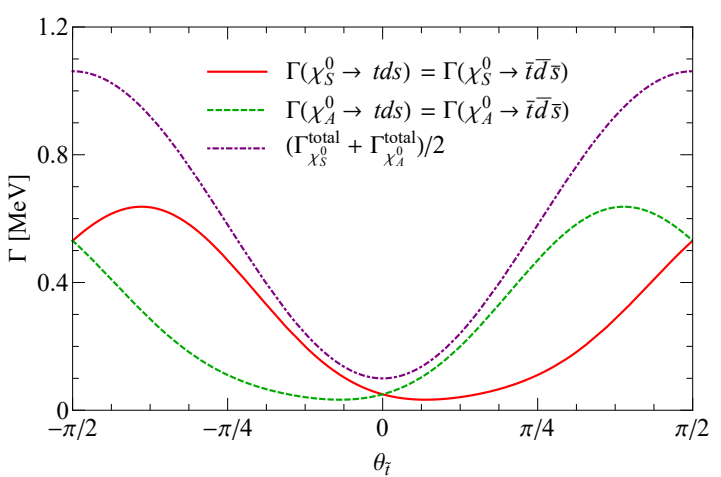

(b)

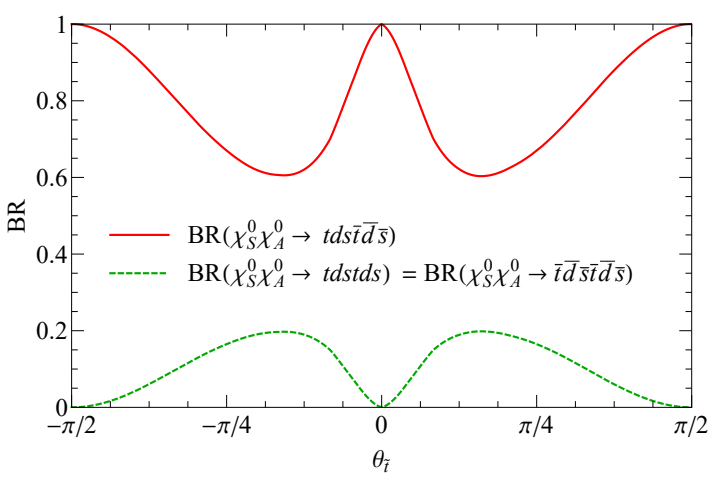

(d)

Figure 5.2: Example figures showing the widths and branching ratios for neutral Higgsinos as functions of the stop mixing angle. Figures 5.2a shows the decay widths for the Higgsino treated as a Dirac state. Figure 5.2b is similar to 5.2a, except the Higgsino is now treated as two Majorana states. Figures 5.2c and 5.2d shows the branching ratios into opposite sign and same sign top final states assuming Higgsino pair production at the LHC. Figure 5.2c treats the pair produced Higgsinos as Dirac particles and is produced by using the partial widths in figure 5.2a together with the narrow width approximation. Figure 5.2d treats the pair produced Higgsinos as Majorana particles and is produced with the assistance of MadGraph. For each of these figures, we have set $\lambda_{312}^{\prime \prime}=1, \tan \beta=40, \mu=500 \mathrm{GeV}, m_{\tilde{t}_{1}}=800 \mathrm{GeV}$, and $m_{\tilde{t}_{2}}=1400 \mathrm{GeV}$.

stop mixing angle. We see that $\chi_{S}^{0}$ and $\chi_{A}^{0}$ have the same widths when there is zero stop mixing, and that their widths are not equal otherwise. The purple dot-dashed line on this figure shows the right-hand side of equation 5.11. This curve matches the purple dot-dashed curve in figure 5.2a exactly, which is the left-hand side of equation 


\subsection{1, as anticipated.}

Next, consider production of neutral Higgsinos at the LHC. This predominantly occurs through an s-channel $Z$ boson which subsequently decays into a pair of Higgsinos. If we consider the Dirac setup, then the $Z$ decays to $\chi_{D}^{0} \bar{\chi}_{D}^{0}$. We can then use the results in figure 5.2a and the narrow width approximation to compute the branching ratio for these two particles to decay into same sign or opposite sign tops, and four down-type quarks. This is presented in figure 5.2c. As expected, when there is zero stop mixing, the pair production of neutral Higgsinos can only produce opposite sign tops in the final state. Allowing stop mixing results in production of same sign tops, with the maximum branching ratio being $\approx 0.2$ for both final states with positive tops and negative tops when $\theta_{\tilde{t}} \approx 0.5$, for this particular choice of parameters.

On the other hand, if we consider the Majorana setup, then the $Z$ decays into $\chi_{S}^{0} \chi_{A}^{0}$ (and never $\chi_{S}^{0} \chi_{S}^{0}$ or $\chi_{A}^{0} \chi_{A}^{0}$ ). If we apply the same logic as in the Dirac case to this situation, then we would conclude that opposite sign and same sign tops would be produced with equal frequency, for all values of $\theta_{\tilde{t}}$. This conclusion is incorrect. The problem is in treating $\chi_{S}^{0}$ and $\chi_{A}^{0}$ as asymptotic states and then applying the narrow width approximation to them. The correct procedure is to calculate the amplitude for a proton proton collision to produce the relevant six-body final state with internal $\chi_{S}^{0}$ and $\chi_{A}^{0}$ particles, and thus explicitly integrate over their widths. While computing this by hand would be challenging, it is possible to perform this computation with MadGraph 5 [74]. The results are shown in figure 5.2d. To compute these curves, the widths set in the MadGraph parameter card are those presented in figure 5.2b. As can be seen, the results from MadGraph, figure 5.2d, agree very well with the results from applying the narrow width approximation to the Dirac states, figure 5.2c.

So far, we have been considering the lowest order in the expansion of the neu- 


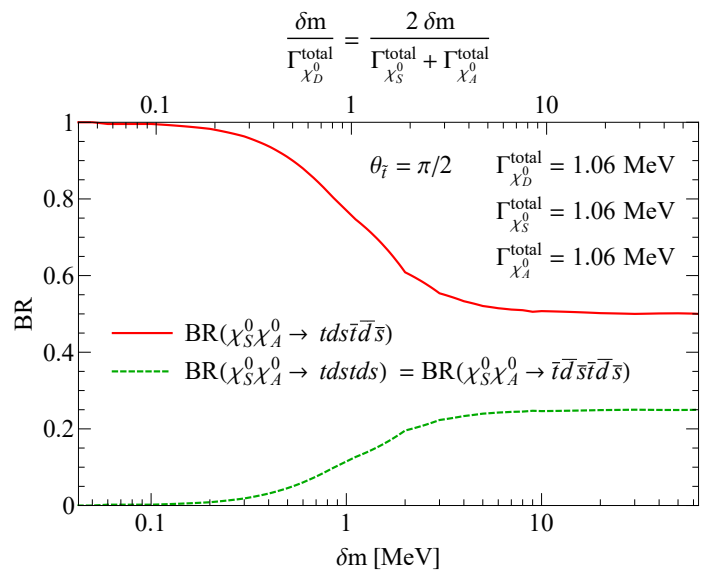

(a)

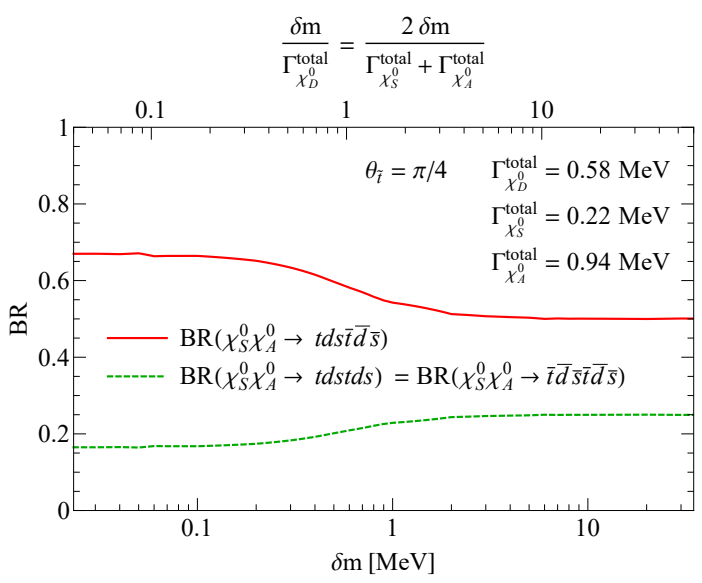

(b)

Figure 5.3: Branching ratios into final states with opposite sign and same sign tops for a $\chi_{S}^{0} \chi_{A}^{0}$ pair produced at the LHC as a function of the mass splitting between the states. Figure 5.3a considers zero stop mixing and figure 5.3b considers maximally mixed stops. The other parameters are set to the same values as in figure 5.2. Both figures have been produced with MadGraph.

tralino mass matrix in the large $\left|M_{1}\right|$ and $M_{2}$ limit. When we include higher order terms in this expansion, the states $\chi_{S}^{0}$ and $\chi_{A}^{0}$ acquire bino and wino components and their masses receive corrections. The masses for the two states then split, by an amount we denote as $\delta m .{ }^{1}$ In the Dirac setup, we can treat $\delta m$ as a perturbation. It then represents the rate at which the particle, $\chi_{D}^{0}$, oscillates into its antiparticle, $\bar{\chi}_{D}^{0}$. As a result, $\chi_{D}^{0} \bar{\chi}_{D}^{0}$ pairs produced at the LHC should decay equally to opposite sign and same sign tops, and four down-type quarks, provided each particle can become sufficiently mixed with its antiparticle before it decays. The rate at which they decay is given by $\Gamma_{\chi_{D}^{0}}^{\text {total }}$, and thus we expect equal production of opposite and same sign tops provided $\delta m \gtrsim \Gamma_{\chi_{D}^{0}}^{\text {total }}$.

We can test this within the Majorana setup by again using MadGraph. Setting the other parameters to the same values as in figure 5.2, we present in figure 5.3 the

\footnotetext{
${ }^{1}$ Loop corrections, to be discussed in section 5.3, also give additional contributions to the masses of the states $\chi_{S}^{0}$ and $\chi_{A}^{0}$. They can also potentially contribute to the mass splitting.
} 
branching ratio of a $\chi_{S}^{0} \chi_{A}^{0}$ pair produced from proton proton collisions as a function of the splitting between their masses, for two different values of stop mixing. In figure $5.3 \mathrm{a}$, we set the stop mixing angle to $\theta_{\tilde{t}}=\pi / 2$ so that the stops do not mix. In this case, the states $\chi_{D}^{0}, \chi_{S}^{0}$, and $\chi_{A}^{0}$ have the same total width. We see that when $\delta m \ll \Gamma_{\chi_{D}^{0}}^{\text {total }}$, only states with opposite sign tops are produced as expected. As $\delta m$ reaches the same order as $\Gamma_{\chi_{D}^{0}}^{\text {total }}$, final states with same sign tops begin to appear. Finally, when $\delta m$ becomes about five times larger than $\Gamma_{\chi_{D}^{0}}^{\text {total }}$, equal amounts of opposite sign and same sign tops are produced. In figure 5.3b, we present a similar plot but now with maximally mixed stops, $\theta_{\tilde{t}}=\pi / 4$. In this case, the widths of $\chi_{D}^{0}$, $\chi_{S}^{0}$, and $\chi_{A}^{0}$ are different. If $\delta m \ll \Gamma_{\chi_{D}^{0}}^{\text {total }}$, then same sign tops are produced due to stop mixing, but opposite sign tops are produced more often. Increasing $\delta m$ causes additional same sign tops to be produced and we see again that equal amounts of opposite sign and same sign tops are produced provided $\delta m$ is larger than about five times the width of the Dirac state.

\subsection{Higgsino masses}

As just mentioned, once higher order terms from the expansion of the neutralino mass matrix are included, the states $\chi_{S}^{0}$ and $\chi_{A}^{0}$ acquire neutral bino and wino components and their masses receive corrections. Similarly, including higher order terms from the expansion of the chargino mass matrix results in $\chi^{-}$acquiring a charged wino component and its mass also receiving corrections. Continuing to work in the large $\left|M_{1}\right|$ and $M_{2}$ limit, we use perturbation theory on the neutralino and chargino mass matrices to determine the leading order shifts to the masses of $\chi_{S}^{0}, \chi_{A}^{0}$, and $\chi^{-}$. We also consider mass contributions from one loop diagrams. Explicitly, we include diagrams involving the top quark-squark loops, bottom quark-squark loops, and the $\gamma, Z, W^{ \pm}$-Higgsino 
loops. ${ }^{2}$ Very similar loop results can be found in [147,148]. Reference [147] provides corrections to the mass splittings between the states and reference [148] provides corrections to the neutralino and chargino mass matrices. The masses for the states are then

$$
\begin{aligned}
& m_{\chi_{S}^{0}}=-\mu-\frac{m_{Z}^{2}\left(M_{1} c_{W}^{2}+M_{2} s_{W}^{2}+\mu\right)\left(1-s_{2 \beta}\right)}{2\left(M_{1}+\mu\right)\left(M_{2}+\mu\right)}+\Sigma_{S}(-\mu) \\
& m_{\chi_{A}^{0}}=\mu-\frac{m_{Z}^{2}\left(M_{1} c_{W}^{2}+M_{2} s_{W}^{2}-\mu\right)\left(1+s_{2 \beta}\right)}{2\left(M_{1}-\mu\right)\left(M_{2}-\mu\right)}+\Sigma_{A}(\mu) \\
& m_{\chi^{-}}=\mu-\frac{m_{W}^{2}\left(\mu+M_{2} s_{2 \beta}\right)}{M_{2}^{2}-\mu^{2}}+\Sigma_{C}(\mu)
\end{aligned}
$$

where $s_{2 \beta}=\sin (2 \beta)$ and $\Sigma_{S}(-\mu), \Sigma_{A}(\mu)$, and $\Sigma_{C}(\mu)$ represent the loop contributions. The neutralino loop functions are

$$
\begin{aligned}
\Sigma_{S}(-\mu)=-\frac{1}{32 \pi^{2}} \operatorname{Re} & \left\{3 y_{t}^{2} \mu\left(B_{1}\left[\frac{\mu^{2}}{Q^{2}}, \frac{m_{t}^{2}}{Q^{2}}, \frac{m_{\tilde{t}_{1}}^{2}}{Q^{2}}\right]+B_{1}\left[\frac{\mu^{2}}{Q^{2}}, \frac{m_{t}^{2}}{Q^{2}}, \frac{m_{\tilde{t}_{2}}^{2}}{Q^{2}}\right]\right)\right. \\
& +3 y_{t}^{2} \sin \left(2 \theta_{\tilde{t}}\right) m_{t}\left(B_{0}\left[\frac{\mu^{2}}{Q^{2}}, \frac{m_{t}^{2}}{Q^{2}}, \frac{m_{\tilde{t}_{1}}^{2}}{Q^{2}}\right]-B_{0}\left[\frac{\mu^{2}}{Q^{2}}, \frac{m_{t}^{2}}{Q^{2}}, \frac{m_{\tilde{t}_{2}}^{2}}{Q^{2}}\right]\right) \\
+ & 3 y_{b}^{2} \mu\left(B_{1}\left[\frac{\mu^{2}}{Q^{2}}, \frac{m_{b}^{2}}{Q^{2}}, \frac{m_{\tilde{b}_{1}}^{2}}{Q^{2}}\right]+B_{1}\left[\frac{\mu^{2}}{Q^{2}}, \frac{m_{b}^{2}}{Q^{2}}, \frac{m_{\tilde{b}_{2}}^{2}}{Q^{2}}\right]\right) \\
+ & 3 y_{b}^{2} \sin \left(2 \theta_{\tilde{b}}\right) m_{b}\left(B_{0}\left[\frac{\mu^{2}}{Q^{2}}, \frac{m_{b}^{2}}{Q^{2}}, \frac{m_{\tilde{b}_{1}}^{2}}{Q^{2}}\right]-B_{0}\left[\frac{\mu^{2}}{Q^{2}}, \frac{m_{b}^{2}}{Q^{2}}, \frac{m_{\tilde{b}_{2}}^{2}}{Q^{2}}\right]\right) \\
+ & \frac{g^{2}}{c_{W}^{2}} \mu\left(B_{1}\left[\frac{\mu^{2}}{Q^{2}}, \frac{\mu^{2}}{Q^{2}}, \frac{m_{Z}^{2}}{Q^{2}}\right]+2 B_{0}\left[\frac{\mu^{2}}{Q^{2}}, \frac{\mu^{2}}{Q^{2}}, \frac{m_{Z}^{2}}{Q^{2}}\right]\right) \\
& \left.+2 g^{2} \mu\left(B_{1}\left[\frac{\mu^{2}}{Q^{2}}, \frac{\mu^{2}}{Q^{2}}, \frac{m_{W}^{2}}{Q^{2}}\right]+2 B_{0}\left[\frac{\mu^{2}}{Q^{2}}, \frac{\mu^{2}}{Q^{2}}, \frac{m_{W}^{2}}{Q^{2}}\right]\right)\right\} \\
\Sigma_{A}(\mu)=\frac{1}{32 \pi^{2}} \operatorname{Re}\{ & 3 y_{t}^{2} \mu\left(B_{1}\left[\frac{\mu^{2}}{Q^{2}}, \frac{m_{t}^{2}}{Q^{2}}, \frac{m_{\tilde{t}_{1}}^{2}}{Q^{2}}\right]+B_{1}\left[\frac{\mu^{2}}{Q^{2}}, \frac{m_{t}^{2}}{Q^{2}}, \frac{m_{\tilde{t}_{2}}^{2}}{Q^{2}}\right]\right) \\
- & 3 y_{t}^{2} \sin \left(2 \theta_{\tilde{t}}\right) m_{t}\left(B_{0}\left[\frac{\mu^{2}}{Q^{2}}, \frac{m_{t}^{2}}{Q^{2}}, \frac{m_{\tilde{t}_{1}}^{2}}{Q^{2}}\right]-B_{0}\left[\frac{\mu^{2}}{Q^{2}}, \frac{m_{t}^{2}}{Q^{2}}, \frac{m_{\tilde{t}_{2}}^{2}}{Q^{2}}\right]\right) \\
+ & 3 y_{b}^{2} \mu\left(B_{1}\left[\frac{\mu^{2}}{Q^{2}}, \frac{m_{b}^{2}}{Q^{2}}, \frac{m_{\tilde{b}_{1}}^{2}}{Q^{2}}\right]+B_{1}\left[\frac{\mu^{2}}{Q^{2}}, \frac{m_{b}^{2}}{Q^{2}}, \frac{m_{\tilde{b}_{2}}^{2}}{Q^{2}}\right]\right)
\end{aligned}
$$

\footnotetext{
${ }^{2}$ See $[144-146]$ for general one loop contributions to both the neutralino and chargino masses.
} 


$$
\begin{aligned}
& -3 y_{b}^{2} \sin \left(2 \theta_{\tilde{b}}\right) m_{b}\left(B_{0}\left[\frac{\mu^{2}}{Q^{2}}, \frac{m_{b}^{2}}{Q^{2}}, \frac{m_{\tilde{b}_{1}}^{2}}{Q^{2}}\right]-B_{0}\left[\frac{\mu^{2}}{Q^{2}}, \frac{m_{b}^{2}}{Q^{2}}, \frac{m_{\tilde{b}_{2}}^{2}}{Q^{2}}\right]\right) \\
& +\frac{g^{2}}{c_{W}^{2}} \mu\left(B_{1}\left[\frac{\mu^{2}}{Q^{2}}, \frac{\mu^{2}}{Q^{2}}, \frac{m_{Z}^{2}}{Q^{2}}\right]+2 B_{0}\left[\frac{\mu^{2}}{Q^{2}}, \frac{\mu^{2}}{Q^{2}}, \frac{m_{Z}^{2}}{Q^{2}}\right]\right) \\
& \left.+2 g^{2} \mu\left(B_{1}\left[\frac{\mu^{2}}{Q^{2}}, \frac{\mu^{2}}{Q^{2}}, \frac{m_{W}^{2}}{Q^{2}}\right]+2 B_{0}\left[\frac{\mu^{2}}{Q^{2}}, \frac{\mu^{2}}{Q^{2}}, \frac{m_{W}^{2}}{Q^{2}}\right]\right)\right\}
\end{aligned}
$$

and the chargino loop function is

$$
\begin{aligned}
\Sigma_{C}(\mu)=\frac{1}{32 \pi^{2}} \operatorname{Re} & \left\{3\left(y_{t}^{2} \sin ^{2} \theta_{\tilde{t}}+y_{b}^{2} \cos ^{2} \theta_{\tilde{t}}\right) \mu B_{1}\left[\frac{\mu^{2}}{Q^{2}}, \frac{m_{b}^{2}}{Q^{2}}, \frac{m_{\tilde{t}_{1}}^{2}}{Q^{2}}\right]\right. \\
& +3\left(y_{t}^{2} \cos ^{2} \theta_{\tilde{t}}+y_{b}^{2} \sin ^{2} \theta_{\tilde{t}}\right) \mu B_{1}\left[\frac{\mu^{2}}{Q^{2}}, \frac{m_{b}^{2}}{Q^{2}}, \frac{m_{\tilde{t}_{2}}^{2}}{Q^{2}}\right] \\
& +3\left(y_{b}^{2} \sin ^{2} \theta_{\tilde{b}}+y_{t}^{2} \cos ^{2} \theta_{\tilde{b}}\right) \mu B_{1}\left[\frac{\mu^{2}}{Q^{2}}, \frac{m_{t}^{2}}{Q^{2}}, \frac{m_{\tilde{b}_{1}}^{2}}{Q^{2}}\right] \\
& +3\left(y_{b}^{2} \cos ^{2} \theta_{\tilde{b}}+y_{t}^{2} \sin ^{2} \theta_{\tilde{b}}\right) \mu B_{1}\left[\frac{\mu^{2}}{Q^{2}}, \frac{m_{t}^{2}}{Q^{2}}, \frac{m_{\tilde{b}_{2}}^{2}}{Q^{2}}\right] \\
& -3 y_{t} y_{b} \sin \left(2 \theta_{\tilde{t}}\right) m_{b}\left(B_{0}\left[\frac{\mu^{2}}{Q^{2}}, \frac{m_{b}^{2}}{Q^{2}}, \frac{m_{\tilde{t}_{1}}^{2}}{Q^{2}}\right]-B_{0}\left[\frac{\mu^{2}}{Q^{2}}, \frac{m_{b}^{2}}{Q^{2}}, \frac{m_{\tilde{t}_{2}}^{2}}{Q^{2}}\right]\right) \\
& -3 y_{t} y_{b} \sin \left(2 \theta_{\tilde{b}}\right) m_{t}\left(B_{0}\left[\frac{\mu^{2}}{Q^{2}}, \frac{m_{t}^{2}}{Q^{2}}, \frac{m_{\tilde{b}_{1}}^{2}}{Q^{2}}\right]-B_{0}\left[\frac{\mu^{2}}{Q^{2}}, \frac{m_{t}^{2}}{Q^{2}}, \frac{m_{\tilde{b}_{2}}^{2}}{Q^{2}}\right]\right) \\
& +\frac{4 g^{2}}{c_{W}^{2}}\left(-\frac{1}{2}+s_{W}^{2}\right)^{2} \mu\left(B_{1}\left[\frac{\mu^{2}}{Q^{2}}, \frac{\mu^{2}}{Q^{2}}, \frac{m_{Z}^{2}}{Q^{2}}\right]+2 B_{0}\left[\frac{\mu^{2}}{Q^{2}}, \frac{\mu^{2}}{Q^{2}}, \frac{m_{Z}^{2}}{Q^{2}}\right]\right) \\
& +4 e^{2} \mu\left(B_{1}\left[\frac{\mu^{2}}{Q^{2}}, \frac{\mu^{2}}{Q^{2}}, 0\right]+2 B_{0}\left[\frac{\mu^{2}}{Q^{2}}, \frac{\mu^{2}}{Q^{2}}, 0\right]\right) \\
& \left.+2 g^{2} \mu\left(B_{1}\left[\frac{\mu^{2}}{Q^{2}}, \frac{\mu^{2}}{Q^{2}}, \frac{m_{W}^{2}}{Q^{2}}\right]+2 B_{0}\left[\frac{\mu^{2}}{Q^{2}}, \frac{\mu^{2}}{Q^{2}}, \frac{m_{W}^{2}}{Q^{2}}\right]\right)\right\} .
\end{aligned}
$$

In these expressions, the sbottom mixing angle $\theta_{\tilde{b}}$ is defined analogously to the stop mixing angle. Additionally, the loop functions $B_{0}\left[p^{2}, m_{1}^{2}, m_{2}^{2}\right]$ and $B_{1}\left[p^{2}, m_{1}^{2}, m_{2}^{2}\right]$ are the two-point Passarino-Veltman functions [149,150] as defined in LoopTools [151, 
152]. ${ }^{3}$ Both functions contain divergent pieces (DP) which are given by

$$
\left.B_{0}\left[p^{2}, m_{1}^{2}, m_{2}^{2}\right]\right|_{\mathrm{DP}}=\Delta \quad \text { and }\left.\quad B_{1}\left[p^{2}, m_{1}^{2}, m_{2}^{2}\right]\right|_{\mathrm{DP}}=-\frac{\Delta}{2}
$$

with $\Delta=2 / \epsilon-\gamma+\log (4 \pi)$. It can then be checked that

$$
-\left.\Sigma_{S}(-\mu)\right|_{\mathrm{DP}}=\left.\Sigma_{A}(\mu)\right|_{\mathrm{DP}}=\left.\Sigma_{C}(\mu)\right|_{\mathrm{DP}}
$$

and this condition implies that the divergences can be absorbed into the renormalized $\mu$ parameter. Concretely, we have used the $\overline{\mathrm{DR}}$ renormalization scheme with renormalization scale $Q$. When we compute the masses, we take this renormalization scale to be $Q=|\mu|[148]$.

\subsection{Constraining Higgsinos at the LHC}

Now that we have discussed some of their properties and calculated their masses, we next study the phenomenology of these almost pure Higgsino neutralinos and charginos. These particles can potentially be pair produced at the LHC, and provided the coupling $\lambda_{3 j k}^{\prime \prime}$ is non-zero and not too small, these particles will decay promptly into final states with at least six quarks. Some of these final states will have same sign tops and this can lead to same sign leptons after the tops decay. By recasting a CMS search which uses this distinctive signature, we will show that parts of the parameter space are beginning to be constrained.

\footnotetext{
${ }^{3}$ Note that the LoopTools definition of the function $B_{1}\left[p^{2}, m_{1}^{2}, m_{2}^{2}\right]$ differs from the definition used in references $[147,148]$ by a minus sign.
} 


\subsubsection{Higgsino decays}

In sections 5.2 and 5.3, we computed fermion masses disregarding if they are positive or negative. For example, if the superpotential parameter $\mu$ is of order a few hundred $\mathrm{GeV}$ and positive, then equations 5.12, 5.13, and 5.14 give that $m_{\chi_{S}^{0}}$ is negative and $m_{\chi_{A}^{0}}$ and $m_{\chi^{-}}$are positive. Of course, the physical masses for these particles are simply given by the absolute value of these equations. While we compute amplitudes with both positive and negative fermion masses, it is easier to discuss the

phenomenology using the physical masses. With this in mind, we will now let $m_{\chi_{S}^{0}}$, $m_{\chi_{A}^{0}}$, and $m_{\chi^{-}}$represent the physical (i.e. positive) masses of these particles. We will also find it convenient to introduce the states $\chi_{1}^{0}$ and $\chi_{2}^{0}$ with

$$
\chi_{1}^{0}=\left\{\begin{array}{ll}
\chi_{S}^{0} & \text { if } m_{\chi_{S}^{0}}<m_{\chi_{A}^{0}} \\
\chi_{A}^{0} & \text { if } m_{\chi_{A}^{0}}<m_{\chi_{S}^{0}}
\end{array} \quad \text { and } \quad \chi_{2}^{0}= \begin{cases}\chi_{S}^{0} & \text { if } m_{\chi_{S}^{0}}>m_{\chi_{A}^{0}} \\
\chi_{A}^{0} & \text { if } m_{\chi_{A}^{0}}>m_{\chi_{S}^{0}}\end{cases}\right.
$$

so that $m_{\chi_{1}^{0}}=\min \left(m_{\chi_{S}^{0}}, m_{\chi_{A}^{0}}\right)$ and $m_{\chi_{2}^{0}}=\max \left(m_{\chi_{S}^{0}}, m_{\chi_{A}^{0}}\right)$.

To analyze the phenomenology of Higgsinos, it is necessary to understand how they decay once they are produced. We therefore list all the possibly relevant decays for $\chi_{S}^{0}, \chi_{A}^{0}$, and $\chi^{-}$. These are

$$
\begin{array}{llll}
\chi_{S}^{0} \rightarrow t d_{j} d_{k} & \chi_{S}^{0} \rightarrow \bar{t} \bar{d}_{j} \bar{d}_{k} & \chi_{S}^{0} \rightarrow \chi_{A}^{0} Z^{*} & \chi_{S}^{0} \rightarrow \chi^{ \pm} W^{\mp *} \\
\chi_{A}^{0} \rightarrow t d_{j} d_{k} & \chi_{A}^{0} \rightarrow \bar{t} \bar{d}_{j} \bar{d}_{k} & \chi_{A}^{0} \rightarrow \chi_{S}^{0} Z^{*} & \chi_{A}^{0} \rightarrow \chi^{ \pm} W^{\mp *}
\end{array}
$$

for the two neutralinos and

$$
\chi^{-} \rightarrow b d_{j} d_{k} \quad \chi^{-} \rightarrow \chi_{S}^{0} W^{-*} \quad \chi^{-} \rightarrow \chi_{A}^{0} W^{-*}
$$


for the chargino, where the $W^{ \pm}$and $Z$ bosons are off-shell. Clearly, the widths for some of these decays will be zero due to kinematic constraints for a given point in parameter space. We calculate the branching ratios for all three particles to decay into their respective final states taking note of the following points.

- As will be seen in section 5.4.4 (and in particular figure 5.4a), excluded points in the parameter space have mass splittings between the neutral Higgsino states typically of order a few $\mathrm{GeV}$. Mass splittings of this size are significantly larger than the decay widths for both $\chi_{S}^{0}$ and $\chi_{A}^{0}$ for the entirety of the parameter space considered. Therefore, we are free to treat $\chi_{S}^{0}$ and $\chi_{A}^{0}$ as asymptotic states, and this results in, for example, the final state with two same sign tops and four down-type quarks being produced as often as the final state with two opposite sign tops and four down-type quarks.

- The first chargino decay is $R$-parity violating and involves an internal stop. There are other $R$-parity violating chargino decays which involve down-type squarks $\left(\chi^{-} \rightarrow \bar{t} \bar{u}_{j} \bar{d}_{k}\right.$ and $\left.\chi^{-} \rightarrow \bar{t} \bar{d}_{j} \bar{u}_{k}\right)$ but we neglect most of these because they are proportional to first or second generation Yukawa couplings. The decay $\chi^{-} \rightarrow \bar{t} \bar{d}_{j} \bar{t}$, which could occur for non-zero $\lambda_{3 j 3}^{\prime \prime}$ and is proportional to the third generation Yukawa couplings, is also not relevant as it is kinematically forbidden for the parameter space that will be considered.

- The off-shell $W^{ \pm *}$ and $Z^{*}$ bosons are decayed into every kinematically available final state. This is straightforward if the final state involves leptons but is slightly more complicated if the final state involves hadronizing quarks. As discussed in [153], perturbative QCD can be used to compute the three-body decay $\chi^{-} \rightarrow \chi^{0} W^{ \pm *} \rightarrow \chi^{0} q \bar{q}^{\prime}$ (where $\chi^{0}$ is either neutralino) provided the mass splitting between the chargino and the neutralino is greater than one or 
two GeV. When the splitting is less than this amount, one instead needs to compute the decay width for $\chi^{-} \rightarrow \chi^{0} \pi^{-}, \chi^{-} \rightarrow \chi^{0} \pi^{-} \pi^{0}, \chi^{-} \rightarrow \chi^{0} \pi^{-} \pi^{0} \pi^{0}$, and $\chi^{-} \rightarrow \chi^{0} \pi^{-} \pi^{+} \pi^{-}$. Precisely, we use the following procedure to handle these types of decays. The width is computed as

$$
\Gamma\left(\chi^{-} \rightarrow \chi^{0} W^{-*}(\text { hadronic })\right)=\max \left[\Gamma\left(\chi^{-} \rightarrow \chi^{0} \pi^{-}\right), \Gamma\left(\chi^{-} \rightarrow \chi^{0} q \bar{q}^{\prime}\right)\right]
$$

where, for simplicity, we only include the one pion final state. Also, the threebody decay includes all possible final state quark pairs and we use constituent quark masses. We then find that for a mass splitting between the chargino and neutralino less than $1.3 \mathrm{GeV}$, the width into a pion is larger, whereas for larger splittings the width into quarks is larger. Reference [153] includes the multiple pion final states and finds a slightly larger cross over mass splitting of $1.5 \mathrm{GeV}$. Finally, the off-shell hadronic $Z$ decays are handled in the same manner, except with $\pi^{0}$ instead of $\pi^{-}$and $q \bar{q}$ instead of $q \bar{q}^{\prime}$.

\subsubsection{Constraining points in the parameter space}

To constrain the parameter space, we recast the CMS search [136], which considers a variety of supersymmetric and non-supersymmetric topologies that produce either same sign tops or four tops. The tops decay and this can lead to same sign leptons, and the search requires this distinctive signature as part of its kinematical cuts. A brief description of the strategy used by this search is given in table 5.1. For this search, we write code to implements its cuts. To check our code, we generate events by using MadGraph 5 [74] to simulate the parton level process, Pythia 8.2 [154] to simulate non-perturbative effects, and Delphes 3 [76] to simulate detector effects. We confirmed that our code can reproduce this search with good accuracy. 


\begin{tabular}{|c|c|c|}
\hline Collaboration & Search & Strategy \\
\hline & & 2 same sign leptons, jets, b-jets, \\
& & $M_{T}^{\text {min }}=\min \left(M_{T}\left(\ell_{1}, \mathrm{MET}\right), M_{T}\left(\ell_{2}, \mathrm{MET}\right)\right)$ \\
CMS & {$[136]$} & where $M_{T}=\operatorname{transverse}$ mass, \\
& & $\mathrm{MET}, H_{T}=\sum_{\text {jets }} p_{T}$ \\
\hline
\end{tabular}

Table 5.1: Experimental search recasted for this analysis.

We use the following procedure to determine constraints from this CMS search. First, the masses of the Higgsino states are determined by using the results in section 5.3. We then use Prospino [84] to compute the production cross sections at the LHC with a centre of mass energy of $13 \mathrm{TeV}$ for all possible Higgsino pairs: $\chi_{S}^{0} \chi_{A}^{0}, \chi_{S}^{0} \chi^{-}$, $\chi_{S}^{0} \chi^{+}, \chi_{A}^{0} \chi^{-}, \chi_{A}^{0} \chi^{+}$, and $\chi^{-} \chi^{+} .{ }^{4}$ The Higgsinos decay as in equations 5.21 and 5.22, and this results in many possible topologies. We then use MadGraph, Pythia, and Delphes to generate events for each topology. By applying the code used to recast the CMS search to these events, we determine the efficiencies for each of the topologies to pass the cuts. The efficiencies are generally small, especially in the signal regions which give constraints, and we therefore generate 250000 events for each topology. By combining these efficiencies with the integrated luminosity, production cross sections, and appropriate branching ratios as computed in section 5.4.1, we determine the expected number of events for each signal region given in the search. These numbers are compared to the $95 \%$ CL upper limit for each signal region which we calculate using the experimental data in the search and standard $\mathrm{CL}_{\mathrm{S}}$ techniques $[85,86]$. We consider a parameter point excluded if the expected number of events for any signal region exceeds its upper limit.

We make the following simplifying assumption when determining the efficien-

\footnotetext{
${ }^{4}$ The Higgsino pairs $\chi_{S}^{0} \chi_{S}^{0}$ and $\chi_{A}^{0} \chi_{A}^{0}$ can also potentially be produced at the LHC but the cross section for doing so is highly suppressed and we therefore ignore these production modes.
} 
cies. Since the mass splittings between the different Higgsino states are only of order a few $\mathrm{GeV}$, any particles created from off-shell $Z$ and $W$ bosons coming from the cascade decays of the Higgsinos are soft enough so that their effects on the efficiencies are negligible. For example, we use the same efficiencies for each of the processes

$$
\begin{aligned}
& p p \rightarrow \chi_{S}^{0} \chi_{A}^{0} \rightarrow t d_{j} d_{k} t d_{j} d_{k}, \\
& p p \rightarrow \chi_{S}^{0} \chi_{A}^{0} \rightarrow Z^{*} \chi_{A}^{0} \chi_{A}^{0} \rightarrow Z^{*} t d_{j} d_{k} t d_{j} d_{k}, \\
& p p \rightarrow \chi^{-} \chi_{A}^{0} \rightarrow W^{-*} \chi_{A}^{0} \chi_{A}^{0} \rightarrow W^{-*} t d_{j} d_{k} t d_{j} d_{k} .
\end{aligned}
$$

We tested this assumption and found that it is valid for mass splittings considered in this work. The necessity of making this assumption is that it significantly reduces the computational running time since we only need to determine efficiencies for the topologies in which both Higgsinos immediately decay by $\lambda_{3 j k}^{\prime \prime}$ into three quarks.

\subsubsection{Parameter scan}

Next, we discuss the relevant parameters. These are $\lambda_{3 j k}^{\prime \prime}$, the parameters in the neutralino and chargino mass matrices: $M_{1}, M_{2}, \mu$, and $\tan \beta$, and the parameters in the stop and sbottom mass matrices: $m_{Q_{3}}, m_{U_{3}}, m_{D_{3}}, A_{t}$, and $A_{b}$. The stop and sbottom mass matrices are given by the standard expressions

$$
m_{\tilde{t}}^{2}=\left(\begin{array}{cc}
m_{Q_{3}}^{2}+m_{t}^{2}+\left(\frac{1}{2}-\frac{2}{3} s_{W}^{2}\right) \cos (2 \beta) m_{Z}^{2} & m_{t}\left(A_{t}-\mu \cot \beta\right) \\
m_{t}\left(A_{t}-\mu \cot \beta\right) & m_{U_{3}}^{2}+m_{t}^{2}+\frac{2}{3} s_{W}^{2} \cos (2 \beta) m_{Z}^{2}
\end{array}\right)
$$


and

$$
m_{\tilde{b}}^{2}=\left(\begin{array}{cc}
m_{Q_{3}}^{2}+m_{b}^{2}+\left(-\frac{1}{2}+\frac{1}{3} s_{W}^{2}\right) \cos (2 \beta) m_{Z}^{2} & m_{b}\left(A_{b}-\mu \tan \beta\right) \\
m_{b}\left(A_{b}-\mu \tan \beta\right) & m_{D_{3}}^{2}+m_{b}^{2}-\frac{1}{3} s_{W}^{2} \cos (2 \beta) m_{Z}^{2}
\end{array}\right)
$$

Since there are many, in principle, free parameters, we scan over the parameter space to find points which are excluded. This is done for both $\lambda_{312}^{\prime \prime}$ and $\lambda_{3 j 3}^{\prime \prime}$. As explained below, the cases with $\lambda_{313}^{\prime \prime}$ and $\lambda_{323}^{\prime \prime}$ are considered together. We use uniform distributions for each of the parameters except $\lambda_{3 j k}^{\prime \prime}$. For this parameter, we instead use a uniform distribution for $\log _{10} \lambda_{3 j k}^{\prime \prime}$. The ranges for these uniform distributions for each parameter except $A_{t}$ and $A_{b}$ are

- $-4<\log _{10} \lambda_{3 j k}^{\prime \prime}<0$,

- $1 \mathrm{TeV}<\left|M_{1}\right|<3 \mathrm{TeV}$,

- $1 \mathrm{TeV}<M_{2}<3 \mathrm{TeV}$,

- $180 \mathrm{GeV}<|\mu|<310 \mathrm{GeV}$,

- $2<\tan \beta<40$,

- $1 \mathrm{TeV}<m_{Q_{3}}<3 \mathrm{TeV}$,

- $1 \mathrm{TeV}<m_{U_{3}}<3 \mathrm{TeV}$,

- $1 \mathrm{TeV}<m_{D_{3}}<3 \mathrm{TeV}$.

To determine appropriate ranges for $A_{t}$ and $A_{b}$, note that if these parameters become too large in magnitude, then there will be charge and colour breaking minimums in the scalar potential deeper than the normal electroweak breaking minimum [155-158]. 
By looking in the $\left|Q_{3}\right|=\left|U_{3}\right|=\left|H_{u}\right|=X$ direction, one can derive the well known bound on $A_{t}[158]$

$$
A_{t}^{2}<3\left(m_{Q_{3}}^{2}+m_{U_{3}}^{2}+m_{H_{u}}^{2}+\mu^{2}\right) .
$$

Similarly, by looking in the $\left|Q_{3}\right|=\left|D_{3}\right|=\left|H_{d}\right|=X$ direction, we derive an analogous bound on $A_{b}$

$$
A_{b}^{2}<3\left(m_{Q_{3}}^{2}+m_{D_{3}}^{2}+m_{H_{d}}^{2}+\mu^{2}\right) .
$$

We use these two inequalities to set the ranges for $A_{t}$ and $A_{b}$, where, for simplicity and also because they can be small compared to our values for $m_{Q_{3}}^{2}, m_{U_{3}}^{2}$, and $m_{D_{3}}^{2}$, we set $m_{H_{u}}^{2} \rightarrow 0$ and $m_{H_{d}}^{2} \rightarrow 0$. With this choice of parameters, the stop masses can range from $770 \mathrm{GeV}$ to $3200 \mathrm{GeV}$ and the sbottom masses can range from $970 \mathrm{GeV}$ to $3015 \mathrm{GeV}$.

The gaugino masses $M_{1}$ and $M_{2}$ are chosen in the TeV range so that the light neutralinos and charginos will be almost pure Higgsinos. Additionally, the stop and sbottom parameters are chosen so that the masses of the stops and sbottoms are mostly in the TeV range as well. In fact, the LHC phenomenology of right-handed stops and $\lambda_{3 j k}^{\prime \prime}$ has been studied in detail in [131] as well as chapter 6 of this thesis. This phenomenology can lead to multiple distinctive signatures. This includes dijets, same sign tops, four tops (with up to three having the same sign), and once the tops decay, same sign leptons and a large number of jets. Importantly, the parameter space excluded by studying this phenomenology does not overlap with the parameter space excluded here. 


\subsubsection{Results}

The results of the parameter scan are shown in figure 5.4. Each of these plots show excluded points in the parameter space projected onto different axes. We show in figure 5.4a the masses for the Higgsinos. In this plot, $\chi_{1}^{0}$ and $\chi_{2}^{0}$, both defined in equation 5.20, represent the lighter and heavier neutralinos. In figure 5.4b we show the excluded points projected onto the $\mu$ and $\lambda_{3 j 3}^{\prime \prime}$ axes. Notice, specifically, that in this figure the vertical axis is $\lambda_{313}^{\prime \prime}$ or $\lambda_{323}^{\prime \prime}$. These two couplings will result in final states which only differ in the production of down versus strange quarks. Since this difference is irrelevant for the recasted search, the results for both cases are the same and so are presented together. Additionally, we did not find any excluded parameter space for the $\lambda_{312}^{\prime \prime}$ coupling. The difference for this coupling is that it results in less b-jet production compared to $\lambda_{313}^{\prime \prime}$ or $\lambda_{323}^{\prime \prime}$. Finally, figure $5.4 \mathrm{c}$ show the values of $M_{1}$ and $M_{2}$ and figures $5.4 \mathrm{~d}$ and $5.4 \mathrm{e}$ show the stop and sbottom masses and their respective mixing angles. ${ }^{5}$

Many features of these figures can be understood as follows. The only final states with non-negligible efficiencies are those which have a pair of same sign tops, and these tops originate from decaying neutralinos. The neutralinos can be produced directly from the parton collision, or they can be produced in the $R$-parity conserving decays of the chargino. In fact, we find that the cross section for $\chi_{S}^{0} \chi_{A}^{0}$ pair production alone is not large enough to constrain any points in the parameter space. That is, for a point to be excluded it is necessary for the chargino to mostly decay into a neutralino and a soft off-shell $W$ boson instead of decaying by the $R$-parity violating $\lambda_{3 j 3}^{\prime \prime}$ coupling into three down-type quarks. This generally happens two different ways: either the parameter point results in a large mass splitting between the chargino and

\footnotetext{
${ }^{5}$ The $\tan \beta$ values of the excluded points coming from the scan are approximately uniformly distributed between small and large values and so we to not make a plot for this parameter.
} 


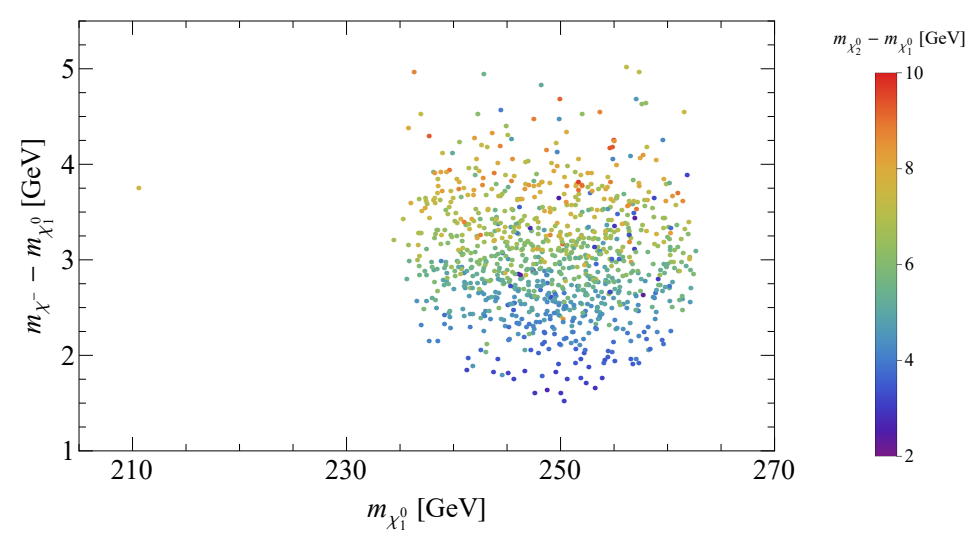

(a)

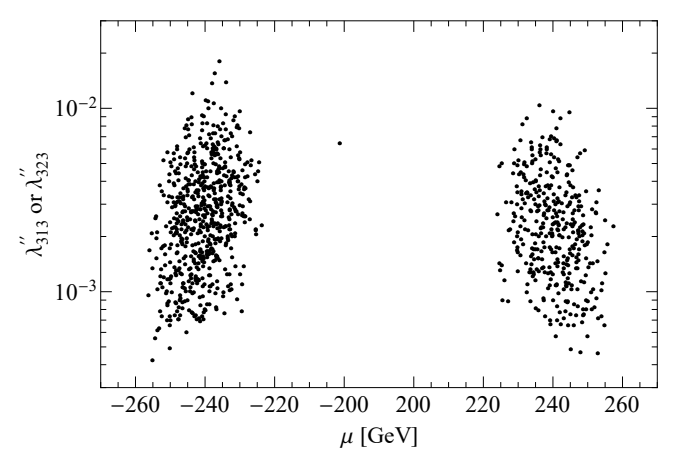

(b)

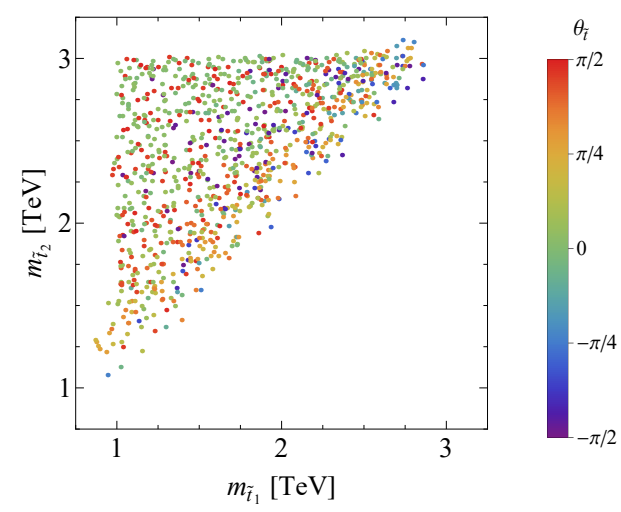

(d)

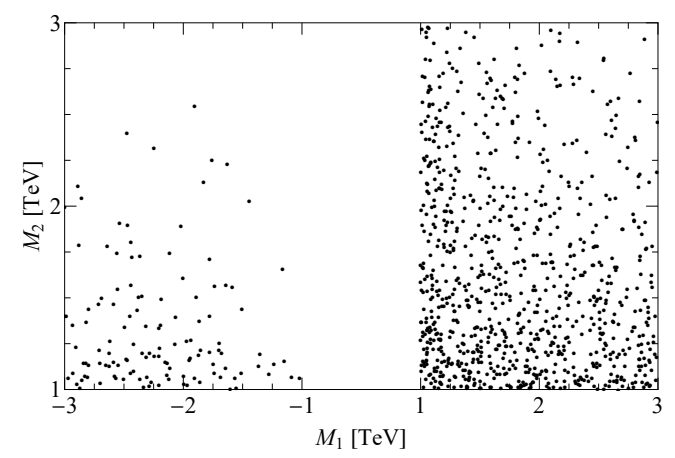

(c)

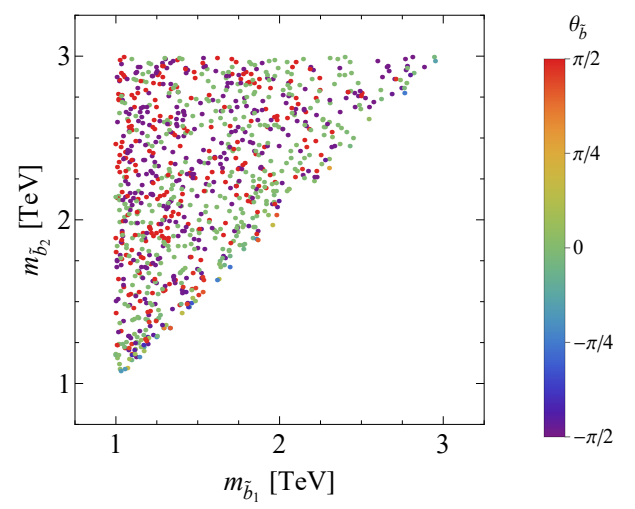

(e)

Figure 5.4: Excluded points found by the parameter scan projected onto different axes. Figure 5.4a shows the Higgsino masses, where $m_{\chi_{1(2)}^{0}}$ is the mass of the lighter (heavier) neutralino, and figures 5.4b, 5.4c, 5.4d, and 5.4e show, respectively, $\lambda_{3 j 3}^{\prime \prime}$ and $\mu, M_{1}$ and $M_{2}$, the stop masses and the stop mixing angle, and the sbottom masses and the sbottom mixing angle. 
the lightest neutralino so as to enhance the $R$-parity conserving decay or it sufficiently suppresses the $R$-parity violating decay of the chargino.

For example, we see in figure 5.4a that the mass splittings between the chargino and the lightest neutralino range between 1.5 and $5.5 \mathrm{GeV}$. The smaller this mass splitting is the more likely the chargino decays into three down-type quarks instead of into the lightest neutralino. Consequently, as the mass splitting approaches its lower limit of $1.5 \mathrm{GeV}$, the mass of the lightest neutralino approaches $250 \mathrm{GeV}$, which maximizes the cross sections times efficiencies. This plot also shows that, with one exception, the lightest Higgsino mass for excluded parameter points found by the scan is greater than $\approx 230 \mathrm{GeV}$ and is less than $\approx 265 \mathrm{GeV}$. Continuing, figure $5.4 \mathrm{~b}$ shows that negative values for $\mu$ are more likely to be excluded compared to positive values. This is simply because negative values for $\mu$ tend to cause larger mass splittings between the chargino and lightest neutralino than positive values. This figure also shows that the $\lambda_{3 j 3}^{\prime \prime}$ values for excluded parameter points fall into a typical range. Too large values of $\lambda_{3 j 3}^{\prime \prime}$ result in the chargino predominately decaying into three down-type quarks. On the other hand, if $\lambda_{3 j 3}^{\prime \prime}$ is too small, then the lightest neutralino becomes too long lived. Since the recasted search relies on promptly decaying particles, we cutoff the exclusion if the lightest neutralino's decay length is longer than $1 \mathrm{~mm}$ [159]. Figure 5.4c shows that positive values of $M_{1}$ are more likely to be excluded than negative values, and that, if $M_{1}$ is positive then smaller values are more likely to be excluded whereas if it is negative then larger absolute values are slightly more likely to be excluded. This figure also shows that excluded points typically have smaller $M_{2}$ values. These regions in the $M_{1}$ and $M_{2}$ parameter space again tend to maximize the mass splitting between the chargino and the lightest neutralino. Figure 5.4d shows that when the excluded point has one light and one heavy stop, the heavier stop is 


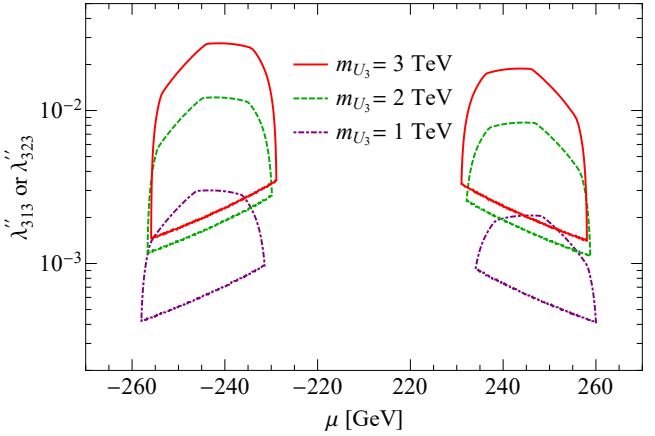

(a)

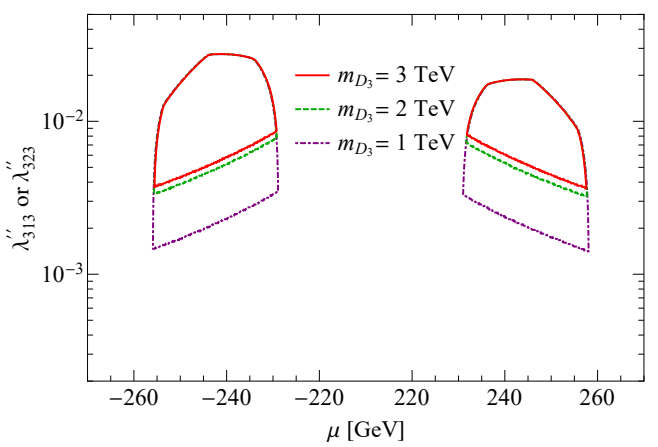

(b)

Figure 5.5: Exclusion curves in the $\mu$ and $\lambda_{3 j 3}^{\prime \prime}$ plane. Figure 5.5a sets $m_{D_{3}}=1 \mathrm{TeV}$ and shows curves for three different vales of $m_{U_{3}}$. Figure $5.5 \mathrm{~b}$ sets $m_{U_{3}}=3 \mathrm{TeV}$ and shows curves for three different vales of $m_{D_{3}}$. The other parameters have been set to $M_{1}=M_{2}=1 \mathrm{TeV}, \tan \beta=10, m_{Q_{3}}=2 \mathrm{TeV}$, and $A_{t}=A_{b}=0$. The region within each curve is excluded.

more likely to be primarily right-handed $\left(\theta_{\tilde{t}}=0\right)$. This configuration suppresses the $R$-parity violating chargino decay. Finally, figure 5.4e shows that when the excluded point has one light and one heavy sbottom, the lighter sbottom is more likely to be primarily right-handed $\left(\theta_{\tilde{b}}=\pi\right.$ or $\left.-\pi\right)$. While this does not directly affect the chargino since its $R$-parity violating decay involves an internal stop, this configuration increases the lightest neutralinos decay width and so increases the chance this particle decays promptly.

To better understand the last points regarding the stop and sbottom masses and mixing angles, consider the exclusions curves presented in figure 5.5. Figure 5.5 a demonstrates the affects the right-handed stop mass $m_{U_{3}}$ has on the excluded parameter region. Larger values of $m_{U_{3}}$ suppress the $R$-parity violating decay of the chargino and so allow larger values of $\lambda_{3 j 3}^{\prime \prime}$ to be excluded. However, larger values also suppress the neutralino decay width and requiring this particle to decay promptly increases the lower limit on $\lambda_{3 j 3}^{\prime \prime}$. Overall, the excluded region shifts upwards with respect to $\lambda_{3 j 3}^{\prime \prime}$ and becomes slightly larger. Figure 5.5b demonstrates the affects 
the right-handed sbottom mass $m_{D_{3}}$ has on the excluded parameter region. Smaller values for $m_{D_{3}}$ increase the neutralino decay width and so allow smaller values of $\lambda_{3 j 3}^{\prime \prime}$ to be excluded. This effect is most noticeable when the right-handed stop mass is large which is why we have set $m_{U_{3}}=3 \mathrm{TeV}$ in this figure.

\subsection{Conclusion}

This chapter studied the LHC phenomenology of pair produced Higgsinos which promptly decay to final states featuring only Standard Model particles due to a single coupling of the form $\lambda_{3 j k}^{\prime \prime}$. To do so, we first examined the properties of Higgsino neutralinos in the limit in which the two Majorana neutralinos can be treated as a single Dirac particle. We showed that the Dirac particle decays with unequal branching ratios to final states $t d_{j} d_{k}$ and $\bar{t} \bar{d}_{j} \bar{d}_{k}$ whereas the Majorana states decay with equal branching ratios to these two final states. Additionally, the total widths of the Majorana states are typically unequal, although their average is equal to the width of the Dirac state as given by equation 5.11. Moreover, in contrast to what is usually expected, equal production of opposite sign and same sign tops from pair produced Higgsinos at the LHC does not occur in the Dirac limit. This can be seen in the Dirac setup by simply applying the narrow width approximation to the Dirac particle and its antiparticle. Showing the same property with two Majorana states is more challenging as it requires computing the amplitude to produce a final state with six quarks treating the two neutralinos as internal particles and thus explicitly integrating over their widths. It is possible to perform this computation with MadGraph and doing so gives the exact same results as in the Dirac setup, as expected. Further, we showed that the usual Majorana behaviour of equal production of opposite and same sign tops is reestablished provided that the mass splitting between the two neutralinos is 
greater than their widths. Since this mass splitting plays an important role, we computed the masses of the Higgsino neutralinos and chargino including both tree-level mixing effects as well as one loop level contributions.

We then proceeded by attempting to constrain the relevant parameter space by studying the LHC phenomenology. First, we examined all possible decay chains after the pair production of Higgsino neutralinos and charginos. Here we noted that the mass splitting between the two neutralinos is typically of order a few $\mathrm{GeV}$ and this is much larger than their widths. Thus, opposite sign and same sign tops will be produced equally often. We then found that by recasting a recent CMS search looking for same sign leptons and multiple b-jets, we were able to exclude individual points in the parameter space. Since there are several free parameters in the model, we scanned over the parameter space using uniform distributions to find a list of excluded points. Our main results are presented in figure 5.4, which show these excluded points projected onto different axes. These plots show that excluded points have Higgsino masses between $\approx 230 \mathrm{GeV}$ and $\approx 265 \mathrm{GeV}$ and that only $\lambda_{3 j 3}^{\prime \prime}$ couplings are excluded and that their values lie between $\approx 3 \times 10^{-4}$ and $\approx 3 \times 10^{-2}$. Finally, these plots emphasize that for a point to be excluded, charginos must undergo an $R$-parity conserving decay into a neutralino and an off-shell $W$ boson. This is to be expected since only neutralinos can decay to tops and only final states with same sign tops have non-negligible efficiencies to pass the CMS search cuts. This point is further demonstrated with regards to the masses and mixing angles for stops and sbottoms in figure 5.5. 


\section{Chapter 6}

\section{Phenomenology of supersymmetric models with a $\mathrm{U}(1)_{R}$ baryon}

\section{number}

\subsection{Introduction}

The previous chapter studied some of the phenomenological consequences of nonzero $\lambda^{\prime \prime}$ couplings in the superpotential. This chapter continues along similar lines but does so from a slightly different viewpoint. Recall that in section 3.9 the idea of $R$-symmetries was introduced. As described in this section, the presence of an $R$-symmetry imposes additional requirements on both the supersymmetry preserving and the soft supersymmetry breaking terms. For example, $R$-symmetric models require Dirac gauginos [160-181] with mass terms of the form given in equation 3.70. Additionally, Dirac gauginos can exhibit supersoft supersymmetry breaking [162] and lead to reduced cross section for the production of squarks [182-184]. It has also been shown that $\mathrm{U}(1)_{R}$ symmetric models have weaker flavour constraints $[164,185]$. Fur- 
thermore, the $\mathrm{U}(1)_{R}$ symmetry can be identified with a lepton or baryon number leading to models where the superpartners have non-standard charges under these symmetries. Such identification can lead to models with unusual structure and phenomenology. For example, if the $\mathrm{U}(1)_{R}$ symmetry is identified with a lepton number, the sneutrino can acquire a significant vacuum expectation value and play the role of the down type Higgs [172-176, 186-188].

The $\mathrm{U}(1)_{R}$ symmetry can alternatively be identified with baryon number $[175$, 189], and we devote this chapter to examining the phenomenology of these types of models. Because this symmetry does not commute with supersymmetry, superpartners have different baryon numbers than their corresponding Standard Model particles which themselves retain their standard baryon number. Under this charge assignment, the standard $R$-parity violating superpotential term of the form $\lambda^{\prime \prime} U^{c} D^{c} D^{c}$ is now baryon number conserving. The bound on such a term is therefore weakened significantly and this can naturally allow large values for the couplings. The LHC phenomenology is then modified as previously demonstrated in chapter 5 , where the decay chains of Higgsinos were considered. More generally, large values of $\lambda^{\prime \prime}$ results in all types of superpartners decaying promptly, making displaced vertices signatures, which are very constraining, less prevalent. Furthermore, an exact $\mathrm{U}(1)_{R}$ would forbid stop decays containing two same sign tops which can lead to same sign leptons, which is also a very constraining signature. Indeed, production of opposite sign and same sign tops also played a crucial role in the previous chapter. In this chapter, this property can now be easily understood in terms of conservation of baryon number.

In this chapter, we first look at how the bounds on the $\lambda^{\prime \prime}$ couplings are modified by the presence of the approximate $\mathrm{U}(1)_{R}$ symmetry. This is presented in section 6.2. In section 6.3 we examine the collider constraints on the model when a single coupling 
of the form $\lambda_{3 j k}^{\prime \prime}$ is important. In particular, we study the constraints coming from stop production and production of the first two generations of squarks. The stop phenomenology is in many cases very similar to the one studied in [131] (see also for example $[132-135,190])$. We conclude this chapter in section 6.4.

\subsection{The model}

The model we consider is an extension of the minimal $R$-symmetric Supersymmetric Standard Model (MRSSM) [164]. It has an approximate $\mathrm{U}(1)_{R}$ symmetry and Dirac gauginos whose mass terms can be written as

$$
\int d^{2} \theta \frac{\mathcal{F}^{\prime \alpha}}{\sqrt{2} M_{*}}\left[c_{1} \mathcal{F}_{\mathcal{B} \alpha} S+c_{2} \mathcal{F}_{\mathcal{W} \alpha}^{b} T^{b}+c_{3} \mathcal{F}_{\mathcal{G} \alpha}^{a} O^{a}\right]+\text { h.c. }
$$

where $\mathcal{F}^{\prime \alpha}=\theta^{\alpha} D^{\prime}$ is a spurion field-strength superfield with a non-zero $D$-term. $S, T^{b}$, and $O^{a}$ are chiral superfields in the adjoint representation of $\mathrm{U}(1)_{\mathrm{Y}}, \mathrm{SU}(2)_{\mathrm{L}}$, and $\mathrm{SU}(3)_{\mathrm{C}}$ respectively, $\mathcal{F}_{i \alpha}(i=\mathcal{B}, \mathcal{W}, \mathcal{G})$ are the Standard Model field-strength superfields, and $M_{*}$ is the supersymmetry breaking mediation scale. The gaugino masses then take the form

$$
M_{i}^{D}=c_{i} \frac{D^{\prime}}{M_{*}}
$$

Further, the standard $\mu$ term is also forbidden by the $\mathrm{U}(1)_{R}$ symmetry, and this requires the addition of the chiral superfields $R_{u}$ and $R_{d}$ to provide masses to the Higgsinos. These new fields have the same gauge numbers as the Higgsinos but different $\mathrm{U}(1)_{R}$ charges. They have bilinear $\mu$-like terms with the Higgs superfields but their scalar components do not acquire vevs. The $\mathrm{U}(1)_{R}$ symmetry can then be identified with baryon number by assigning the right-handed quark superfields $R$-charge $2 / 3$ and the left-handed quark superfields $R$-charge $4 / 3$. The charge assignments of 


\begin{tabular}{|c|c|}
\hline Fields & $R$-charge \\
\hline$H_{u, d}$ & 0 \\
\hline$R_{u, d}$ & 2 \\
\hline$U^{c}, D^{c}$ & $2 / 3$ \\
\hline$S, T, O$ & 0 \\
\hline$Q$ & $4 / 3$ \\
\hline$L, E^{c}$ & 1 \\
\hline
\end{tabular}

Table 6.1: $R$-charge assignment of chiral superfields of the model.

the remaining superfields are shown in table 6.1. Under this symmetry all the Standard Model particles have their usual baryon number. However, superpartners have non-standard baryon numbers. For example, the right-handed squarks have baryon number $2 / 3$ and thus are diquarks, while the left-handed squarks have baryon number $4 / 3$ and the gauginos baryon number 1 . Gauge symmetries and the $\mathrm{U}(1)_{R}$ symmetry lead to the following superpotential

$$
\begin{aligned}
W= & y_{u} Q H_{u} U^{c}-y_{d} Q H_{d} D^{c}-y_{e} L H_{d} E^{c}+\mu_{u} H_{u} R_{d}+\mu_{d} R_{u} H_{d} \\
& +\lambda_{u}^{t} H_{u} T R_{d}+\lambda_{d}^{t} R_{u} T H_{d}+\lambda_{u}^{s} S H_{u} R_{d}+\lambda_{d}^{s} S R_{u} H_{d}+\frac{1}{2} \lambda_{i j k}^{\prime \prime} U_{i}^{c} D_{j}^{c} D_{k}^{c}
\end{aligned}
$$

where $T=T^{b} \sigma^{b} / 2$. This superpotential is equal to the superpotential of the MRSSM to which the standard $R$-parity violating term of the form $\lambda^{\prime \prime} U^{c} D^{c} D^{c}$ has been added. ${ }^{1}$ Beside gaugino masses, the soft SUSY breaking terms include non-holomorphic scalar masses, $B_{\mu}$ like terms, and a linear term for $S$

$$
V_{\text {soft }}=\sum_{\Phi} M_{\Phi}^{2}|\Phi|^{2}+\left[B_{\mu} H_{u} H_{d}+\frac{1}{2} b_{S} S^{2}+\frac{1}{2} b_{T} T^{2}+\frac{1}{2} b_{O} O^{2}+f_{S} S+\text { h.c. }\right] .
$$

Various tri-linear terms are also allowed by the symmetries of the model but can be suppressed [175]. In addition, the $f_{S}$ term needs to be small to avoid destabilizing

\footnotetext{
${ }^{1}$ This term violates the standard $R$-parity but not the $\mathrm{U}(1)_{R}$ symmetry defined in table 6.1 .
} 
the hierarchy. On general grounds, the $\mathrm{U}(1)_{R}$ symmetry cannot remain an exact symmetry of the theory. The breaking will manifest itself at least through the gravitino mass. This breaking will then unavoidably be communicated to the Standard Model sector through anomaly mediation [26,27]. Majorana gaugino mass terms and tri-linear $A$ terms will in this case be generated with size of order

$$
M \sim A \sim \frac{1}{16 \pi^{2}} m_{3 / 2}
$$

\subsubsection{Bounds on $\lambda^{\prime \prime}$}

The bounds on the $\lambda^{\prime \prime}$ couplings come in our model from the same sources as in the $R$ Parity Violating Supersymmetric Standard Model, namely flavour violating processes and baryon number violating processes.

The situation in the RPVMSSM goes as follows. The flavour violating processes put severe constraints on products of $\lambda^{\prime \prime}$ s with different flavour structures while baryon number violating processes can impose strong constraints on the $\lambda^{\prime \prime}$ individually [19]. The baryon number violating processes that put the most stringent bounds are proton decay, neutron antineutron oscillation, and double nucleon decay. The proton decay constraint can be avoided if we assume that lepton number is conserved and that the gravitino is heavier than the proton, leaving neutron antineutron oscillation and double nucleon decay which are still very constraining for many of the $\lambda^{\prime \prime}$ s, with the constraint on $\lambda_{112}^{\prime \prime}$ being the strongest. One approach to satisfy both the flavour violating and baryon number violating constraints is to assume a minimal flavour violating $(\mathrm{MFV})$ structure for the $\lambda_{i j k}^{\prime \prime}[137]$. This leads to very small couplings and the LHC phenomenology is then characterized by displaced vertices. Another approach to avoid the bounds is to assume that in the mass eigenstate basis only 
one coupling of the form $\lambda_{3 j k}^{\prime \prime}$ is large while the $\lambda^{\prime \prime}$ s with different flavour structures are very suppressed. The bounds are then easily satisfied. Single stop production becomes relevant at the LHC, and neutralinos can decay promptly via an off-shell stop to a top and two jets. This phenomenology was explored in [131]. The difficulty in such a scenario is to build a flavour model that leads in the mass basis to a large $\lambda_{3 j k}^{\prime \prime}$ coupling but a very small $\lambda_{112}^{\prime \prime}$ coupling.

\subsubsection{Bounds from baryon number violating processes}

In the model we consider, baryon number is violated only by the small $\mathrm{U}(1)_{R}$ breaking terms coming from anomaly mediation which are proportional to the gravitino mass. Constraints from baryon number violating processes are then potentially weaker than in the RPVMSSM. However, if the gravitino is lighter than the proton, the proton can decay to a gravitino and a kaon. This process proceeds through a $\lambda_{112}^{\prime \prime}$ coupling and is the same as in the RPVMSSM, leading to a bound of [19]

$$
\lambda_{112}^{\prime \prime} \lesssim 6 \times 10^{-15}\left(\frac{m_{\tilde{q}}}{1 \mathrm{TeV}}\right)^{2}\left(\frac{m_{3 / 2}}{1 \mathrm{eV}}\right)
$$

When the gravitino is heavier than the proton, the bounds from neutron antineutron oscillation and double nucleon decay still apply. The best experimental limit on neutron antineutron oscillation comes from the non-observation of ${ }^{16} \mathrm{O}$ decay to various final states with multiple pions and omega particles at SuperKamiokande [191]. This process receives tree-level contributions from diagrams of the form shown in figure 6.1a. This leads to a bound on $\lambda_{11 i}^{\prime \prime}$ which is somewhat model dependent as the diagram requires flavour mixing mass insertions on the squark lines. It also requires the insertion of a Majorana mass term for the gluino which we take to be given by anomaly mediation: $M_{3}=3 \alpha_{s} m_{3 / 2} / 4 \pi$. The amplitude for this process can be 


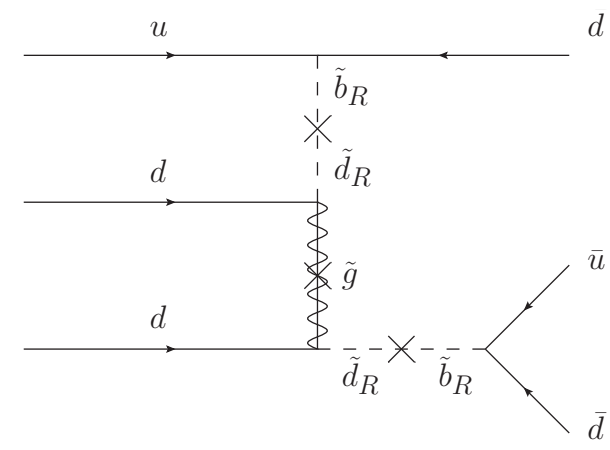

(a)

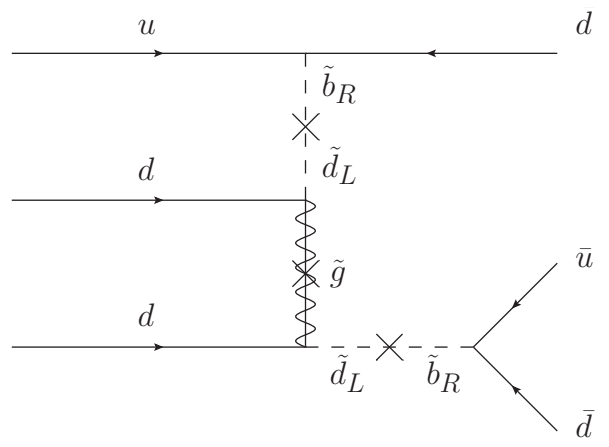

(b)

Figure 6.1: Diagrams leading to neutron antineutron oscillation. Flavour changing insertions are needed on the squark lines and a Majorana mass insertion is needed on the gluino line. (a) shows a diagram with flavour changing insertions of the righthanded squarks. (b) shows a diagram requiring a left-right squark mixing which is further suppressed by the gravitino mass.

estimated to be [137]

$$
M_{n-\bar{n}} \sim 4 \pi \alpha_{s}\left(\lambda_{11 i}^{\prime \prime}\right)^{2} \frac{\left(\delta_{i 1}^{R R}\right)^{2}}{m_{\tilde{q}}^{4}} \frac{M_{3}}{\left(M_{3}^{D}\right)^{2}} \Lambda^{6}
$$

where $\delta_{i j}^{R R}$ is the ratio of the flavour non-diagonal elements of the right-handed downtype squark mass matrix to the flavour diagonal ones and $\Lambda$ is the characteristic scale for the neutron matrix elements which is expected to be close to the QCD scale. Taking $\alpha_{s}=0.12$, we find a bound of the form [137]

$$
\lambda_{11 i}^{\prime \prime} \lesssim 2 \times 10^{-5}\left(\frac{1}{\delta_{i 1}^{R R}}\right)\left(\frac{M_{3}^{D}}{1 \mathrm{TeV}}\right)\left(\frac{1 \mathrm{GeV}}{m_{3 / 2}}\right)^{1 / 2}\left(\frac{m_{\tilde{q}}}{1 \mathrm{TeV}}\right)^{2}\left(\frac{250 \mathrm{MeV}}{\Lambda}\right)^{3}
$$

If for some reason the effect of the flavour mixing in the right-handed squark mass matrix is small, which could happen if, for example, this matrix follows an MFV pattern ${ }^{2}$ then the process needs to involve left-right squark mixing (see figure 6.1b). In

\footnotetext{
${ }^{2}$ With an MFV structure, there exists a basis where both the right-handed squark matrix and the gauge Yukawa interactions involving down-type quarks are flavour diagonal.
} 


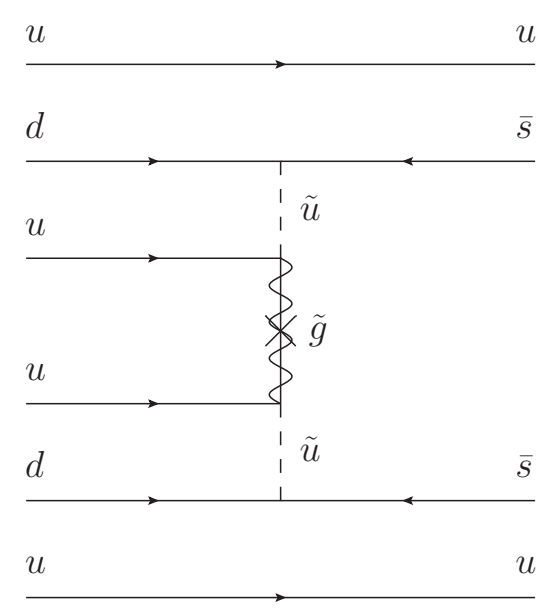

Figure 6.2: Diagram mediating the $p p \rightarrow K^{+} K^{+}$process.

the limit of an exact $\mathrm{U}(1)_{R}$, these mixings, which come from $A$ terms, are forbidden. They are however expected to be generated with a size proportional to the gravitino mass once $\mathrm{U}(1)_{R}$ breaking effects are taken into account. Taking the anomaly mediation value for the $A$ terms, the bound becomes

$$
\lambda_{11 i}^{\prime \prime} \lesssim 2\left(\frac{1}{y_{1 i}^{d}}\right)\left(\frac{M_{3}^{D}}{1 \mathrm{TeV}}\right)\left(\frac{1 \mathrm{GeV}}{m_{3 / 2}}\right)^{3 / 2}\left(\frac{m_{\tilde{q}}}{1 \mathrm{TeV}}\right)^{4}\left(\frac{250 \mathrm{MeV}}{\Lambda}\right)^{3}
$$

where $y_{i j}^{d}$ is the down-type Yukawa matrix, and we see that order one $\lambda^{\prime \prime}$ become easily allowed.

The bound coming from double nucleon decay is more independent from flavour physics as it can proceed through a diagram such as the one showed in figure 6.2 which does not require flavour mixing on the squark lines. The diagram on the other hand still requires the insertion of a gluino Majorana mass term. The best limit on this process also comes from the non-observation of ${ }^{16} \mathrm{O}$ decay to ${ }^{14} \mathrm{C} K^{+} K^{+}$at Superkamiokande [192]. The bound on the partial lifetime is found to be $1.7 \times 10^{32}$ years. A rough estimate for the amplitude can be obtained in a similar way to the 
$n-\bar{n}$ process $[137,193]$. It leads to a bound on $\lambda_{112}^{\prime \prime}$ of the form

$$
\lambda_{112}^{\prime \prime} \lesssim 2 \times 10^{-4}\left(\frac{M_{3}^{D}}{1 \mathrm{TeV}}\right)\left(\frac{1 \mathrm{GeV}}{m_{3 / 2}}\right)^{1 / 2}\left(\frac{m_{\tilde{q}}}{1 \mathrm{TeV}}\right)^{2}\left(\frac{150 \mathrm{MeV}}{\tilde{\Lambda}}\right)^{5 / 2}
$$

where $\tilde{\Lambda}$ is the hadronic scale which is hard to estimate and introduces significant uncertainty on the bound. It is expected to be suppressed compared to $\Lambda_{\mathrm{QCD}}$ due to nucleon repulsion $[137,193]$.

\subsubsection{Bounds from flavour physics}

Flavour physics also puts strong bounds on the $\lambda^{\prime \prime}$ parameters. The bounds are on products of two $\lambda^{\prime \prime}$ s with different flavour structures $[19,138]$. For example, there are loop diagrams that contribute to $\epsilon_{K}$ and $\Delta m_{K}$, leading to a bound of the form [138]

$$
\sqrt{\left|\operatorname{Im}\left(\lambda_{i 23}^{\prime \prime} \lambda_{i 13}^{\prime \prime *}\right)^{2}\right|} \lesssim 2.8 \times 10^{-3}\left(\frac{m_{\tilde{u}_{i}}}{1 \mathrm{TeV}}\right)
$$

from $\epsilon_{K}$ while $\Delta m_{K}$ gives

$$
\sqrt{\left|\operatorname{Re}\left(\lambda_{i 23}^{\prime \prime} \lambda_{i 13}^{\prime \prime *}\right)^{2}\right|} \lesssim 4.6 \times 10^{-2}\left(\frac{m_{\tilde{u}_{i}}}{1 \mathrm{TeV}}\right)
$$

There are also strong bounds on $\lambda_{i 23}^{\prime \prime} \lambda_{i 12}^{\prime \prime *}$ from $B$-mixing and from bounds on $\operatorname{BR}\left(B^{ \pm} \rightarrow\right.$ $\left.\phi \pi^{ \pm}\right)$. These bounds can be satisfied by having only one of the $\lambda^{\prime \prime}$ s sizable in the mass eigenstate basis. Whether or not this can be easily achieved depends on the structure of the flavour physics. For example, it might be possible to arrange for one of the $\lambda^{\prime \prime}$ to be dominant in the gauge basis, but when rotating to the mass basis other flavour structures will be generated. If the rotation has the same structure as the CKM matrix a $\lambda_{312}^{\prime \prime}$ coupling of order one in the gauge basis is allowed by flavour physics constraints provided the squarks have masses in the $\mathrm{TeV}$ range [138]. How- 
ever, in order to satisfy the bound on $\lambda_{112}^{\prime \prime}$ from equation 6.10 , the rotation of the right-handed up squarks from the gauge to the mass basis must induce a suppression of $\sim 10^{-4}$, and a CKM like structure will be insufficient. If $\lambda_{313}^{\prime \prime}$ is dominant in the gauge basis, $K-\bar{K}$ mixing constrains this coupling to be $\lesssim 0.1$, and in this case a CKM-like rotation structure for the up squarks will put $\lambda_{112}^{\prime \prime}$ close to the bound of equation 6.10.

\subsubsection{Spectrum and parameter space}

In view of the strong constraints on the $\lambda^{\prime \prime}$ couplings, we focus from now on models where only a single coupling of the form $\lambda_{3 j k}^{\prime \prime}$ is important. The relevant features of the phenomenology will crucially depend on the size of this coupling and on the spectrum. For example, for large $\lambda_{3 j k}^{\prime \prime}$ single stop production can be important while a smaller coupling leads to pair production being dominant. Large $\lambda_{3 j k}^{\prime \prime}$ couplings will also lead to the prompt decay of neutralinos to a top quark and two jets, leading to a distinct phenomenology from the displaced vertices characteristic of the small RPV coupling case.

In the limit where the $\mathrm{U}(1)_{R}$ symmetry is exact there is a distinction between neutralinos and antineutralinos. ${ }^{3}$ One of them has baryon number 1 and decays to $t j j$, while the other has baryon number -1 and decays to $\bar{t} j j$. In this case, the decay of a stop will always involve opposite sign tops: $\tilde{t} \rightarrow t \bar{\chi}_{0} \rightarrow t \bar{t} j j$. However, in the presence of Majorana mass terms for the gauginos, the Dirac neutralinos split into two Majorana states which can both decay to either $t j j$ or $\bar{t} j j$. This is important for the phenomenology as in this case there will be a signature with two same sign

\footnotetext{
${ }^{3}$ The following discussion regarding Dirac versus Majorana neutralinos (which is also continued in section 6.3.1.3) is similar to the one presented in section 5.2. However, we repeat it here in detail so that each chapter can be read independently. Also, in contrast to the RPVMSSM, the Dirac case in this chapter is well motivated as it follows from the limit in which the $R$-symmetry is unbroken.
} 
leptons. We can see how this works by looking at a bino LSP interacting with the stop through the following potential

$$
M_{1}^{D} \tilde{S} \tilde{B}+\frac{1}{2} M_{1} \tilde{B} \tilde{B}-\frac{2 \sqrt{2}}{3} g^{\prime} \tilde{t}_{R}^{\dagger}\left(t_{R} \tilde{B}\right)+\lambda_{323}^{\prime \prime} \tilde{t}_{R}\left(b_{R} s_{R}\right)+\text { h.c. }
$$

where $M_{1}$ is a small Majorana mass term for the bino. The mass eigenstates are two pseudo-Dirac states [194] given by ${ }^{4}$

$$
\begin{aligned}
& \chi_{1}^{B}=i \frac{1}{\sqrt{2}}(\tilde{B}-\tilde{S}) \\
& \chi_{2}^{B}=\frac{1}{\sqrt{2}}(\tilde{B}+\tilde{S})
\end{aligned}
$$

with corrections of order $M_{1} / M_{1}^{D}$. The masses of the two eigenstates are given by $m_{1}^{B}=M_{1}^{D}-M_{1} / 2$ and $m_{2}^{B}=M_{1}^{D}+M_{1} / 2$ to leading order. In terms of the mass eigenstates, the potential can then be written as

$$
\frac{m_{1}^{B}}{2} \chi_{1}^{B} \chi_{1}^{B}+\frac{m_{2}^{B}}{2} \chi_{2}^{B} \chi_{2}^{B}+i \frac{2 g^{\prime}}{3} \tilde{t}_{R}^{\dagger} t_{R} \chi_{1}^{B}-\frac{2 g^{\prime}}{3} \tilde{t}_{R}^{\dagger} t_{R} \chi_{2}^{B}+\lambda_{323}^{\prime \prime} \tilde{t}_{R}\left(b_{R} s_{R}\right)+\text { h.c. }
$$

The decay of the stop can proceed via an on-shell $\chi_{1}^{B}$ or $\chi_{2}^{B}$ as shown in figure 6.3. In the case of a decay to $t t j j$, the two amplitudes have opposite sign and interfere destructively, while for the decay to $t \bar{t} j j$, the amplitudes have the same sign and add. Therefore, for a mass splitting smaller than the width of $\chi_{1}^{B}$ and $\chi_{2}^{B}$, decay chains with same sign tops are suppressed whereas for a larger mass splitting they occur as often as opposite sign tops. In our study of the LHC phenomenology we will compute the bound on squark masses as a function of the $\lambda_{3 j k}^{\prime \prime}$ for bino and Higgsino-up LSP. For gravitino masses slightly above $\sim 1 \mathrm{GeV}$, for which the bound from proton decay

\footnotetext{
${ }^{4}$ In this chapter we rephase the antisymmetric combination of Weyl spinors by a factor of $i$ so that the mass terms for $\chi_{1}^{B}$ and $\chi_{2}^{B}$ have the same sign (cf. equation 5.9).
} 


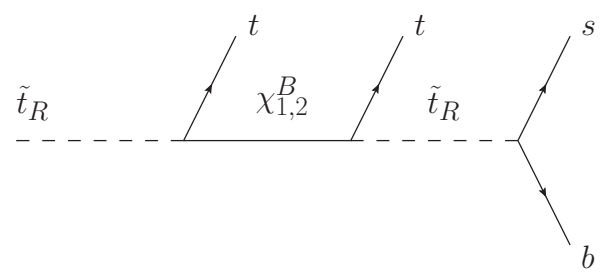

Figure 6.3: Decay of the stop through the two on-shell pseudo-Dirac states $\chi_{1}^{B}$ and $\chi_{2}^{B}$. When the mass difference between the two states is smaller than the width, the diagram with $\chi_{1}^{B}$ cancels the one with $\chi_{2}^{B}$.

to gravitino does not apply, the mass splitting between the pseudo-Dirac neutralino states is small enough to be ignored for most processes, except for the stop decay. A mass splitting of order $1 \mathrm{GeV}$ is much larger than the typical neutralino width and, as a consequence, decay chains with same sign tops will occur. We will also show bounds for the case where the $\mathrm{U}(1)_{R}$ symmetry is nearly exact with no same sign top signatures. This last case requires a very low $m_{3 / 2}$, which might be difficult to achieve, but could have interesting consequences for cosmology [195].

\subsection{Collider constraints}

In this section, we constrain the parameter space of the model by using a variety of LHC searches. We focus on two different scenarios. The first scenario is resonant stop production together with stop pair production. The second scenario is pair production of the first and second generations of squarks. From here on out we simply refer to this scenario as squark production. For both scenarios, we consider the cases in which the $\mathrm{U}(1)_{R}$ symmetry is either strictly preserved or, alternatively, broken. As mentioned above, whether or not the $\mathrm{U}(1)_{R}$ symmetry is broken changes the phenomenology. 


\subsubsection{Placing limits on stops}

\subsubsection{Stop production}

The main phenomenological novelty of the model is the presence in the superpotential of the term

$$
\frac{1}{2} \lambda_{3 j k}^{\prime \prime} U_{3}^{c} D_{j}^{c} D_{k}^{c}
$$

which can only contain stops that are right-handed. Consequently, we concentrate on the production of right-handed stops, which we simply refer to as stops for the remainder of this chapter. The left-handed stop, which does not mix with the righthanded one as it possesses a different $R$-charge, is assumed to be decoupled.

If any of $\lambda_{312}^{\prime \prime}, \lambda_{313}^{\prime \prime}$, or $\lambda_{323}^{\prime \prime}$ is non-zero, resonant stop production can potentially take place at the LHC. For example, turning on $\lambda_{312}^{\prime \prime}$ will result in the partonic level processes $d s \rightarrow \tilde{t}^{*}$ and $\bar{d} \bar{s} \rightarrow \tilde{t}$, provided that the stop is not too heavy. Precisely, the partonic level cross section for $d_{j} d_{k} \rightarrow \tilde{t}^{*}$ is [196]

$$
\hat{\sigma}\left(d_{j} d_{k} \rightarrow \tilde{t}^{*}\right)=\frac{\pi}{6} \frac{\left|\lambda_{3 j k}^{\prime \prime}\right|^{2}}{m_{\tilde{t}}^{2}} \delta\left(1-m_{\tilde{t}}^{2} / \hat{s}\right),
$$

where $\hat{s}$ is the partonic centre of mass energy. Due to the valance down quark, the cross section to produce $\tilde{t}^{*}$ is generally much larger than that to produce $\tilde{t}$ (although if only $\lambda_{323}^{\prime \prime}$ is non-zero than $\tilde{t}^{*}$ and $\tilde{t}$ are produced in roughly equal amounts). Additionally, due to the small content of strange and bottom within the proton, stop production through $\lambda_{312}^{\prime \prime}$ is larger than that through $\lambda_{313}^{\prime \prime}$, which is itself larger than that through $\lambda_{323}^{\prime \prime}$, assuming equal values for $\lambda_{312}^{\prime \prime}, \lambda_{313}^{\prime \prime}$, and $\lambda_{323}^{\prime \prime}$. We use MadGraph $5[74]$ to calculate the leading order ( $\mathrm{LO})$ cross section at centre of mass energies 
of 8 and $13 \mathrm{TeV}$ for resonant stop production (summing both $\tilde{t}^{*}$ and $\tilde{t}$ ) turning on $\lambda_{312}^{\prime \prime}, \lambda_{313}^{\prime \prime}$, and $\lambda_{323}^{\prime \prime}$ one at a time. ${ }^{5}$ In this fashion, all constraints placed throughout this section assume only a single $\lambda_{3 j k}^{\prime \prime}$ is non-zero. Our limits are then conservative compared to the case where multiple $\lambda_{3 j k}^{\prime \prime}$ are non-zero.

Naturally, the LO cross section will be corrected by next-to-leading order (NLO) QCD effects. The NLO cross section for single stop production has been calculated in Ref. [198]. There, K-factors for each of the $\lambda_{3 j k}^{\prime \prime}$ are presented for stop masses between 200 and $800 \mathrm{GeV}$ at a centre of mass energy of $14 \mathrm{TeV}$. It was found that the K-factors varied between approximately 1.2 and 1.4. To account for this, we simply multiply the LO cross sections computed with MadGraph by a constant K-factor of 1.3 for all stop masses. Figure 6.4 shows the resulting cross sections where each $\lambda_{3 j k}^{\prime \prime}$ has been set individually to one.

As in the MSSM, stops will also be produced in pairs. However, due to the $\lambda_{3 j k}^{\prime \prime}$ coupling, there are new diagrams that contribute. These diagrams consist of two $\lambda_{3 j k}^{\prime \prime}$ vertices, two initial state quarks, and a t-channel quark. Couplings of order one can give significant contributions to the cross sections. For example, using MadGraph to compute the LO pair production cross section for $200 \mathrm{GeV}$ stops at $13 \mathrm{TeV}$, we find a $20 \%$ increase when $\lambda_{312}^{\prime \prime}$ is set to one compared to when it is zero. However, as far as we know, NLO corrections have not been computed for these new diagrams. Moreover, for $\lambda_{3 j k}^{\prime \prime}$ of order one, single top production dominates the exclusion in most of the parameter space. For these reasons, we choose not to include this new contribution to our stop pair production cross section. Instead, we compute the cross section for this process using NLL-fast [83,199,200] and NNLL-fast [199-202] for centre of mass energies of 8 and $13 \mathrm{TeV}$, respectively. We verify the results using Prospino [84]. Our

\footnotetext{
${ }^{5}$ To simulate collisions, we used the Mathematica package FeynRules 2.0 [197] to produce our own MRSSM MadGraph models, one with the $\mathrm{U}(1)_{R}$ symmetry preserved and another with the symmetry broken.
} 


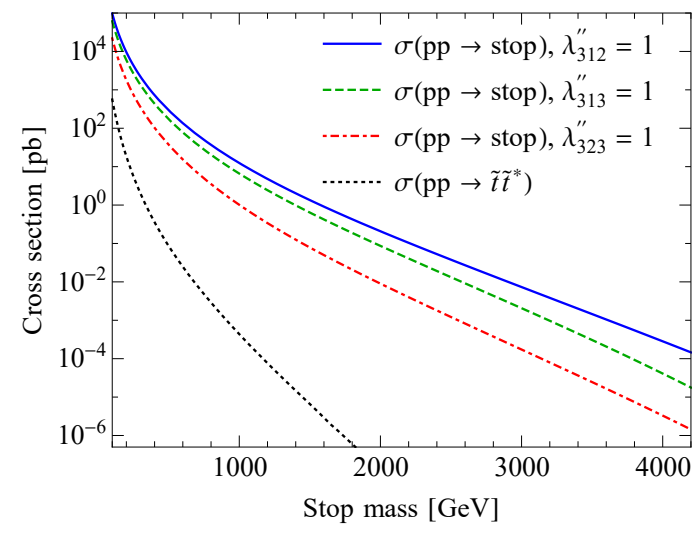

(a) $8 \mathrm{TeV}$

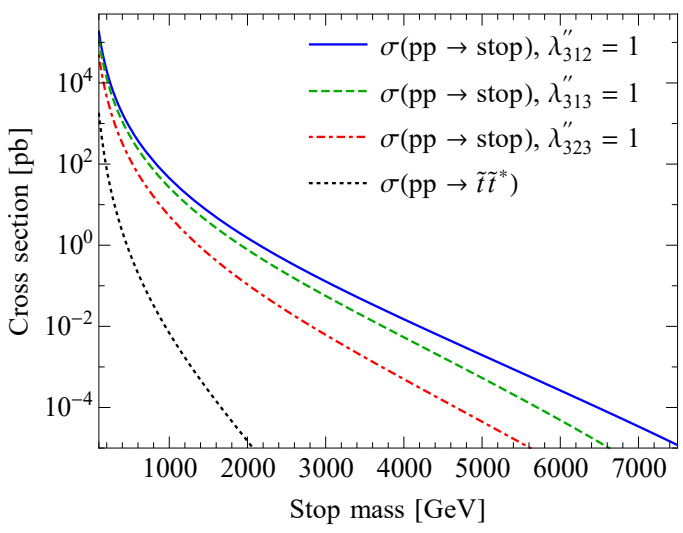

(b) $13 \mathrm{TeV}$

Figure 6.4: Stop production at centre of mass energy of 8 and $13 \mathrm{TeV}$. Here $\sigma(\mathrm{pp} \rightarrow$ stop) stands for $\sigma\left(\mathrm{pp} \rightarrow \tilde{t}^{*}\right)+\sigma(\mathrm{pp} \rightarrow \tilde{t})$. For resonant stop production, only one $\lambda_{3 j k}^{\prime \prime}$ is non-zero at a time.

limits from stop pair production are then conservative, particularly when the $\lambda_{3 j k}^{\prime \prime}$ are of order one. The cross section is also shown in figure 6.4. As can be seen, resonant stop production is quite a bit larger than stop pair production for any of the $\lambda_{3 j k}^{\prime \prime}$ set to unity.

\subsubsection{Stop LSP}

If the stop is the LSP, then it will decay directly into two quarks through the $\lambda_{3 j k}^{\prime \prime}$ coupling with a branching ratio of one. In this situation, there are two processes of interest

$$
p p \rightarrow \tilde{t}^{*} \rightarrow d_{j} d_{k}
$$

(2) $p p \rightarrow \tilde{t}^{*} \tilde{t} \rightarrow d_{j} d_{k} \bar{d}_{j} \bar{d}_{k}$

where the final state quarks depend on which one $\lambda_{3 j k}^{\prime \prime}$ is non-zero. The process $(1)$ also includes its charge conjugated version. We now constrain the parameter space using these two processes. 
Let us focus on the first process. This case is sensitive to dijet searches performed at the LHC. We examined many of these searches and selected the following ones to recast: [203-205] from ATLAS and [206,207] from CMS. The procedures used to recast these searches are described below. Notably, each one of these searches is independent of the flavour of the final state quarks as they do not utilize b-tagging. We also considered the ATLAS dijet search [208] which does utilize b-tagging but found that the exclusion limits did not improve. Particularly, search [205] provides stronger limits than [208]. The reason for this is that even though [208] requires a b-tagged jet, the limits on the cross section times branching ratio times acceptance between [205] and [208] are comparable. However, requiring a b-tagged jet results in the acceptance for [208] being about half that of [205], thus making it less constraining.

Both the ATLAS and CMS experiments have developed special techniques to place limits on low mass resonances decaying to dijets. The ATLAS technique is known as Trigger-object Level Analysis (TLA) and was implemented in [204] to constrain masses below $1.1 \mathrm{TeV}$. The CMS technique is known as data scouting and was implemented in searches $[206,207]$ to constrain masses below $1.6 \mathrm{TeV}$. The low mass region is experimentally difficult due to a combination of the limited bandwidth available to record events to disk and the large Standard Model multijet rate. Either a large fraction of events must be discarded or stringent triggers must be used in order to keep the amount of recorded data to an acceptable level. However, both options limit the statistical power of the search. The TLA and data scouting approach is to record only the portion of the event data, such as jet four-momenta, needed to perform the dijet search. By doing so, event sizes can be reduced to $5 \%(2 \%)$ of what they would normally be for ATLAS [204] (CMS [206]). This allows for more statistics and hence stronger limits. 
To recast ATLAS dijet searches, we followed the procedure within Appendix A of [203] to set limits on models of new physics with Gaussian resonances. First, for each search we chose a selection of stop masses $M$ to sample. Then, for each $M$, we used MadGraph to generate 10000 events of resonant stop production with the stop subsequently decaying into quarks. The events were given to Pythia 8.2 [154] to simulate non-perturbative effects and then fed into Delphes $3[76]$ for detector simulation. The package HepMC2 [209] was used to interface between Pythia and Delphes. Next, code was written to implement the kinematic cuts. The cuts for each search were

$$
\begin{aligned}
& \text { [203]: }\left|y_{j_{1}}\right|<2.8,\left|y_{j_{2}}\right|<2.8, p_{T_{j_{1}}}>50 \mathrm{GeV}, p_{T_{j_{2}}}>50 \mathrm{GeV} \\
& \left|\Delta y_{j_{1} j_{2}}\right|<1.2, m_{j_{1} j_{2}}>250 \mathrm{GeV}, 0.8 M<m_{j_{1} j_{2}}<1.2 M, \\
& \text { [204] : }\left|\eta_{j_{1}}\right|<2.8,\left|\eta_{j_{2}}\right|<2.8, p_{T_{j_{1}}}>185 \mathrm{GeV}, p_{T_{j_{2}}}>85 \mathrm{GeV} \text {, } \\
& \left|\Delta y_{j_{1} j_{2}}\right|<\left\{\begin{array}{l}
0.6 \text { if } 425 \mathrm{GeV}<m_{G}<550 \mathrm{GeV}, \\
1.2 \text { if } 550 \mathrm{GeV}<m_{G}<1100 \mathrm{GeV},
\end{array}\right. \\
& 0.8 M<m_{j_{1} j_{2}}<1.2 M, \\
& {[205]: p_{T_{j_{1}}}>440 \mathrm{GeV}, p_{T_{j_{2}}}>60 \mathrm{GeV}} \\
& \left|\Delta y_{j_{1} j_{2}}\right|<1.2, m_{j_{1} j_{2}}>1100 \mathrm{GeV}, 0.8 M<m_{j_{1} j_{2}}<1.2 M,
\end{aligned}
$$

where, for the two leading jets $j_{1}$ and $j_{2}: y_{j_{1}}$ and $y_{j_{2}}$ are their rapidities, $\eta_{j_{1}}$ and $\eta_{j_{2}}$ are their pseudorapidities, $p_{T_{j_{1}}}$ and $p_{T_{j_{2}}}$ are their transverse momenta, $\Delta y_{j_{1} j_{2}}$ is the difference between their rapidities, and $m_{j_{1} j_{2}}$ is their invariant mass. The cut $0.8 M<m_{j_{1} j_{2}}<1.2 M$ is designed to remove any long tails in the reconstructed $m_{j_{1} j_{2}}$ distribution which has been assumed to be Gaussian. The acceptance for a search is then the fraction of the events to pass its cuts. The acceptances are shown in the top 


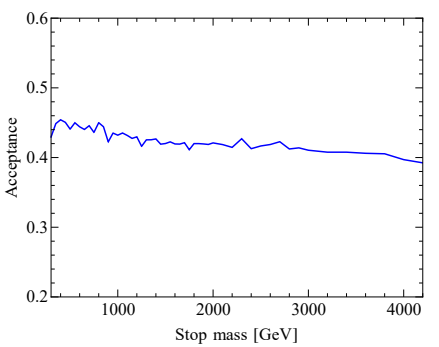

(a) [203]

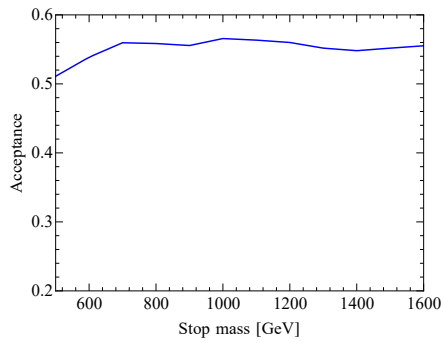

(d) $[206]$

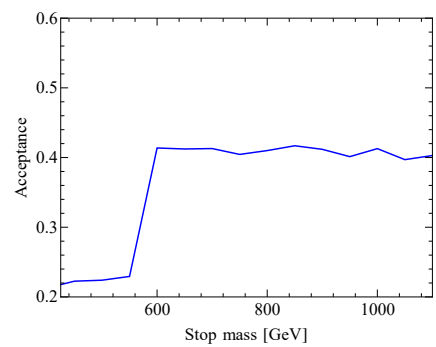

(b) $[204]$

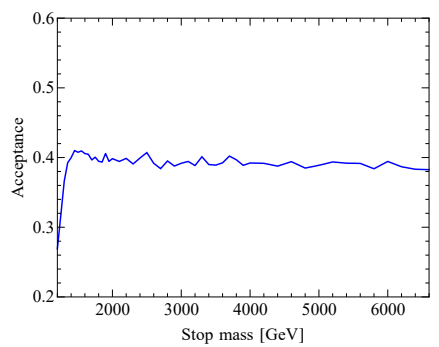

(c) $[205]$

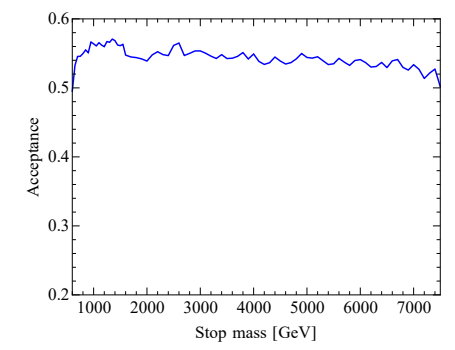

(e) [207]

Figure 6.5: Acceptances for the dijet searches recasted in this analysis. Top row: ATLAS searches. Bottom row: CMS searches.

row of figure 6.5. Additionally, events that passed had their values of $m_{j_{1} j_{2}}$ recorded in a histogram. A Gaussian distribution was then fit to the histogram and the standard deviation, $\sigma_{G}$, and mean, $m_{G}$, were determined. Finally, each search provided $95 \%$ CL upper limits on the cross section times branching ratio times acceptance as a function of $m_{G}$ for different values of $\sigma_{G} / m_{G}$. We found that the vast majority of $\sigma_{G} / m_{G}$ values fell between 0.05 and 0.07 .

Recasting CMS dijet searches followed a similar procedure up until applying the cuts. A major component of the cuts centred around reconstructing two "wide jets". The two leading jets served as the seeds for the two wide jets and the four-momentum of any other jet would be added to the closest leading jet if the two were separated by less than $\Delta R=1.1$. Then, for a stop with mass $M$, the cuts for each search were

$$
[206]: H_{T}=\sum_{j} p_{T_{j}}>250 \mathrm{GeV}, \Delta \phi_{j_{1} j_{2}}>\pi / 3,\left|\Delta \eta_{J_{1} J_{2}}\right|<1.3, m_{J_{1} J_{2}}>390 \mathrm{GeV}
$$




$$
\begin{aligned}
{[207]: H_{T}=\sum_{j} p_{T_{j}}>\left\{\begin{array}{l}
250 \mathrm{GeV} \text { if } 0.6 \mathrm{TeV}<M<1.6 \mathrm{TeV}, \\
800 \mathrm{GeV} \text { if } 1.6 \mathrm{TeV}<M<7.5 \mathrm{TeV},
\end{array}\right.} \\
\left|\Delta \eta_{J_{1} J_{2}}\right|<1.3, \\
m_{J_{1} J_{2}}>\left\{\begin{array}{l}
453 \mathrm{GeV} \text { if } 0.6 \mathrm{TeV}<M<1.6 \mathrm{TeV}, \\
1058 \mathrm{GeV} \text { if } 1.6 \mathrm{TeV}<M<7.5 \mathrm{TeV},
\end{array}\right.
\end{aligned}
$$

where $H_{T}$ is the scalar sum of the transverse momenta of all the jets, $\Delta \phi_{j_{1} j_{2}}$ is the azimuthal angle between the two leading jets, and, for the two wide jets $J_{1}$ and $J_{2}$, $\Delta \eta_{J_{1} J_{2}}$ is the difference between their pseudorapidities and $m_{J_{1} J_{2}}$ is their invariant mass. Once again, the acceptance for a search is the fraction of the events to pass its cuts. The acceptances are shown in the bottom row of figure 6.5. Both CMS searches provided 95\% CL upper limits on the cross section times branching ratio times acceptance for dijets originating from two quarks.

It is also possible to constrain the parameter space using the second process outlined at the beginning of this section, stop pair production with subsequent decay into four quarks. As a matter of fact, there have been several experimental searches looking for exactly this signature. These include searches [210-212] from ATLAS and [213] from CMS. We directly read off the limits on the cross section times branching ratio as a function of stop mass. For stops decaying only into quarks, the most powerful search is [212], which is independent of the flavour of the final state quarks. That is, it does not explicitly require b-tagged jets. This is in contrast to the other searches, [210,211] both require b-jets while [213] provides different limits depending on whether or not b-jets are produced. As a result, the limits on the stop mass are the same for all three $\lambda_{3 j k}^{\prime \prime}$, once again assuming a branching ratio of one.

Combining the limits from the two types of searches considered in this section, 


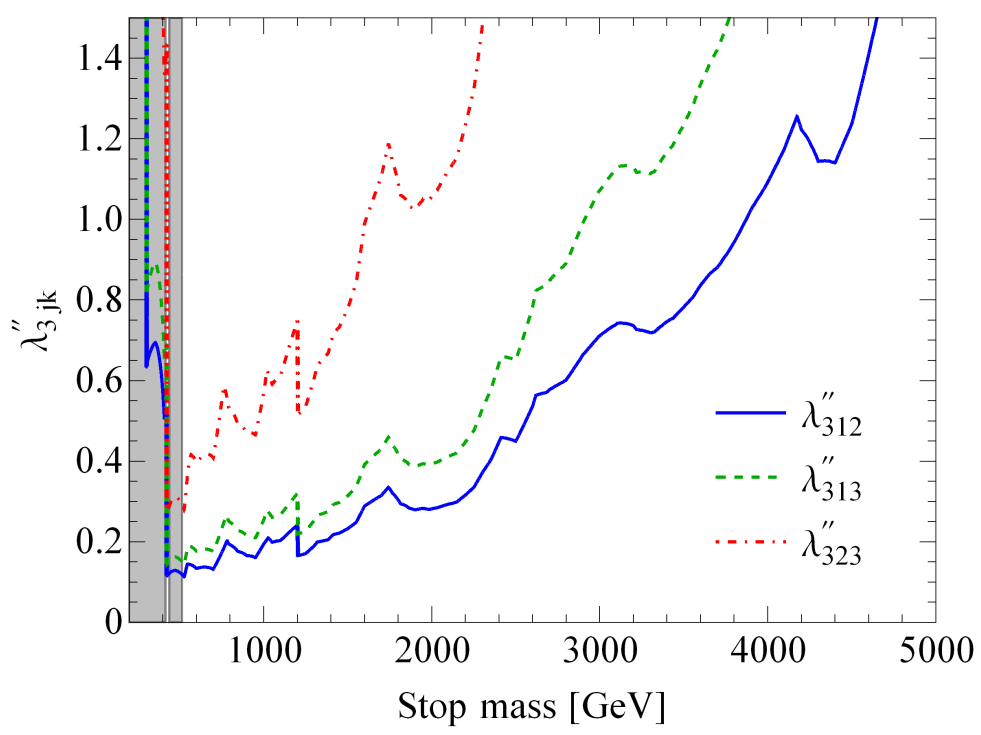

Figure 6.6: Exclusion plot for an LSP stop. The area above each curve is excluded by dijet searches. Additionally, the grey area is excluded by stop pair production with subsequent decay into four quarks. As explained in the text, it applies equally to all $\lambda_{3 j k}^{\prime \prime}$.

we constrain the $\lambda_{3 j k}^{\prime \prime}$ and stop mass parameter space. The result is shown in figure 6.6. The small white band of stop masses slightly above $400 \mathrm{GeV}$ fails to be excluded due to an upward fluctuation of the signal in the search [212]. Interestingly, for each $\lambda_{3 j k}^{\prime \prime}$ exclusion curve resulting from the dijet searches, at least a portion of its left edge happens to fall directly in this small unexcluded range. Future searches will likely close this gap. Disregarding this feature for a moment, we see that stop masses up to 3870, 2910, and $1610 \mathrm{GeV}$ are excluded for $\lambda_{312}^{\prime \prime}, \lambda_{313}^{\prime \prime}$, and $\lambda_{323}^{\prime \prime}$ set to one, respectively. A similar plot is also presented within Ref. [131]. For comparison, Ref. [131] found that stop masses up to 3150, 2830, and 1500 (plus a small region between 1730 and 1870) $\mathrm{GeV}$ are excluded for $\lambda_{312}^{\prime \prime}, \lambda_{313}^{\prime \prime}$, and $\lambda_{323}^{\prime \prime}$ set to one, respectively. 


\subsubsection{Neutralino LSP}

If the LSP is a neutralino, additional phenomenological possibilities emerge. However, the stop is assumed to be right-handed, and as such couples only to the bino or the Higgsino-up. Therefore, we focus on two different possibilities: the LSP neutralino is essentially pure bino or essentially pure Higgsino-up. Naturally, for the Higgsino-up case, there is also an accompanying chargino with approximately the same mass. The next lightest neutralino or chargino is then taken to be heavier than the stop. This assures no cascade decays between neutralinos which would complicate the possible decay topologies. This type of spectrum, while not necessarily the most general, allows us to investigate the parameter space in a fairly straightforward and intuitive manner. We mention here that throughout this section and the section in which we constrain squarks, 6.3.2.2, we set $\tan \beta=10$.

In general, there are now three different possibilities for how the stop can decay: $\tilde{t}^{*} \rightarrow d_{j} d_{k}, \tilde{t}^{*} \rightarrow \bar{t} \chi^{0}$, or $\tilde{t}^{*} \rightarrow \bar{b} \chi^{-}$. The first decay mode occurs, as before, through the $\lambda_{3 j k}^{\prime \prime}$ coupling. For the last two decay modes, $\chi^{0}$ refers to the lightest neutralino and $\chi^{-}$is the lightest chargino. Of course, if the LSP is a bino neutralino, only the first two decays will have non-zero branching ratios. On the other hand, if the Higgsino-up neutralino is the LSP then all three decay modes will occur. For both cases, we compute the branching ratios for the stop into each of the possible final states. For example, figure 6.7 presents the branching ratios for a $600 \mathrm{GeV}$ stop as a function of $\lambda_{312}^{\prime \prime}$, with the neutralino mass set to $200 \mathrm{GeV}$. As the figure shows, the stop decays mostly into dijets for $\lambda_{312}^{\prime \prime}$ of order one, while the other modes quickly begin to dominate for lesser values.

We now discuss the decay modes for the neutralinos and charginos, starting with the latter. Along with the normal decay of the chargino into the neutralino and 


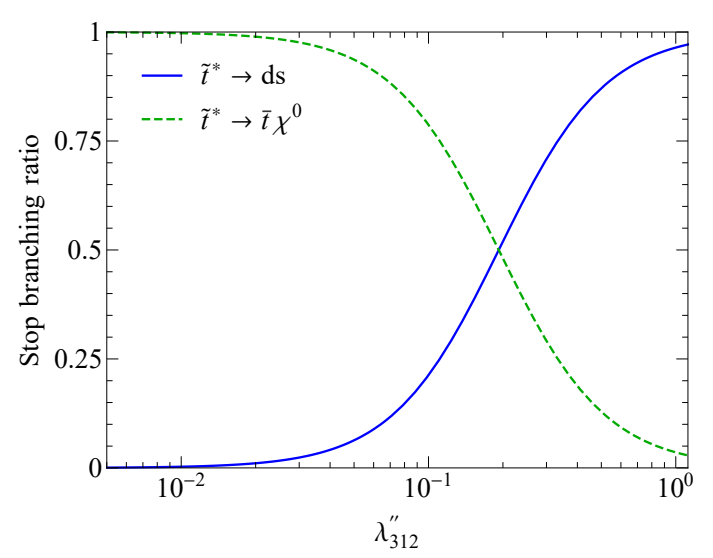

(a) Bino neutralino

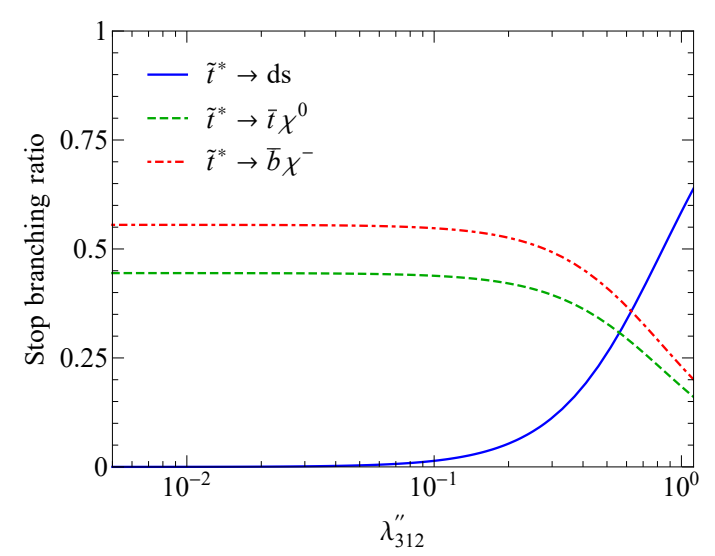

(b) Higgsino-up neutralino

Figure 6.7: Branching ratios for a $600 \mathrm{GeV}$ stop as a function of of $\lambda_{312}^{\prime \prime}$. For both plots, the neutralino mass has been set to $200 \mathrm{GeV}$.

a $W$ boson, the $\lambda_{3 j k}^{\prime \prime}$ coupling allows for an additional decay into three quarks through an off-shell stop. Precisely, this new decay is $\chi^{-} \rightarrow b \tilde{t}^{*} \rightarrow b d_{j} d_{k}$. For the type of spectrum under consideration, the splitting between the chargino and neutralino is quite small. It then follows that the decay of the chargino into a neutralino and an off-shell $W$ boson is highly phase space suppressed. As a result, for essentially all values of $\lambda_{3 j k}^{\prime \prime}$ and stop masses considered in this analysis, the RPV decay for the chargino dominates. We explicitly checked this by computing the branching ratios for the chargino and confirmed that this is indeed the case. Unless otherwise stated, we consider the chargino to decay into three quarks with a branching ratio of one. ${ }^{6}$

Due to the $\lambda_{3 j k}^{\prime \prime}$ coupling, the neutralino is also unstable and will decay into three quarks. This decay also occurs within the RPVMSSM but there is now an important difference. As explained in section 6.2.2, our model has Dirac neutralinos which split into two pseudo-Dirac states once the small $\mathrm{U}(1)_{R}$ breaking is taken into account. For Dirac neutralinos, there is only a single decay mode while for pseudo-

\footnotetext{
${ }^{6}$ This choice of parameters differs from the one made in chapter 5 where the mass splitting between the chargino and neutralino was large enough so that the chargino's RPC decay competed with its RPV decay.
} 
Dirac neutralinos there are two. Specifically, the decay mode for Dirac neutralinos is $\chi^{0} \rightarrow t \tilde{t}^{*} \rightarrow t d_{j} d_{k}$ (the antineutralino decay is $\bar{\chi}^{0} \rightarrow \bar{t} \tilde{t} \rightarrow \bar{t} \bar{d}_{j} \bar{d}_{k}$ ). Pseudo-Dirac neutralinos can decay by $\chi^{0} \rightarrow t \tilde{t}^{*} \rightarrow t d_{j} d_{k}$ or $\chi^{0} \rightarrow \tilde{t} \tilde{t} \rightarrow \bar{t} \bar{d}_{j} \bar{d}_{k}$. For a mass splitting, proportional to the scale of the $\mathrm{U}(1)_{R}$ breaking, larger than the width, the two decay modes for the pseudo-Dirac neutralinos become equally relevant. The neutralinos will then behave similarly to the standard Majorana neutralinos of the RPVMSSM. Conversely, for mass splitting smaller than the width, the neutralino behaves as a purely Dirac state with a single decay mode.

To demonstrate this feature, consider $600 \mathrm{GeV}$ stops decaying through approximately $200 \mathrm{GeV}$ binos with $\lambda_{312}^{\prime \prime}=1$. In figure 6.8 we show the partial decay widths and corresponding branching ratios for the stop as a function of the bino Majorana mass term. The branching ratios for the two different decays become equal when the Majorana mass is about five times the decay width of the neutralinos. ${ }^{7}$ We thoroughly explore the parameter space and find equivalent behaviour for the opposite sign and same sign decay widths. However, we note that this result crucially depends on the neutralinos being produced on-shell. If the stops decay through off-shell neutralinos, then the propagators of the neutralinos are not inversely proportional to their widths. In this case, the equality of branching ratios occurs when the mass splitting is comparable to the Dirac mass. ${ }^{8}$

To understand the phenomenological significance of this, suppose a $\tilde{t}^{*}$ is pro-

${ }^{7}$ Figure 6.8 is very similar to figure 5.3. The main conclusion of equal production of same sign and opposite sign tops when the mass splitting between the two Higgsinos is about five time their widths can be seen from either figure.

${ }^{8}$ Although this discussion has been in terms of a bino LSP, similar results also hold for a Higgsinoup LSP. However, as a Majorana mass term for the Higgsino-up is not necessarily generated, the mass splitting between the two pseudo-Dirac Higgsino-up neutralinos results from a combination of Majorana gaugino masses for the bino and wino and mixing. For example, setting $\lambda_{312}^{\prime \prime}=1$, $m_{\tilde{t}}=600 \mathrm{GeV}, \mu_{u}=200 \mathrm{GeV}, M_{1}^{D}=M_{2}^{D}=10 \mathrm{TeV}$, and $M_{1}$ and $M_{2}$ to their anomaly mediation masses, we find equal branching ratios for opposite sign and same sign tops resulting from stop decays for $m_{3 / 2} \gtrsim 7 \times 10^{-2} \mathrm{GeV}$. If the Dirac masses for the bino and wino are lowered to $1 \mathrm{TeV}$, then equal branching ratios occur for $m_{3 / 2} \gtrsim 3 \times 10^{-4} \mathrm{GeV}$. 


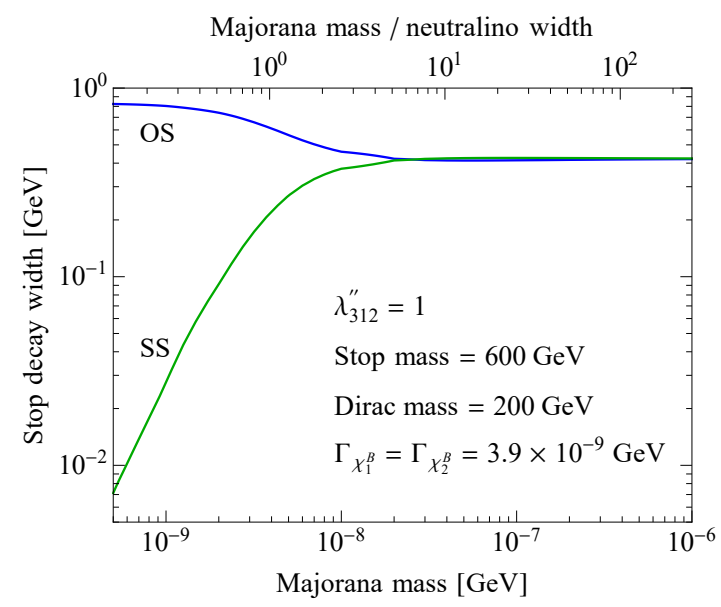

(a)

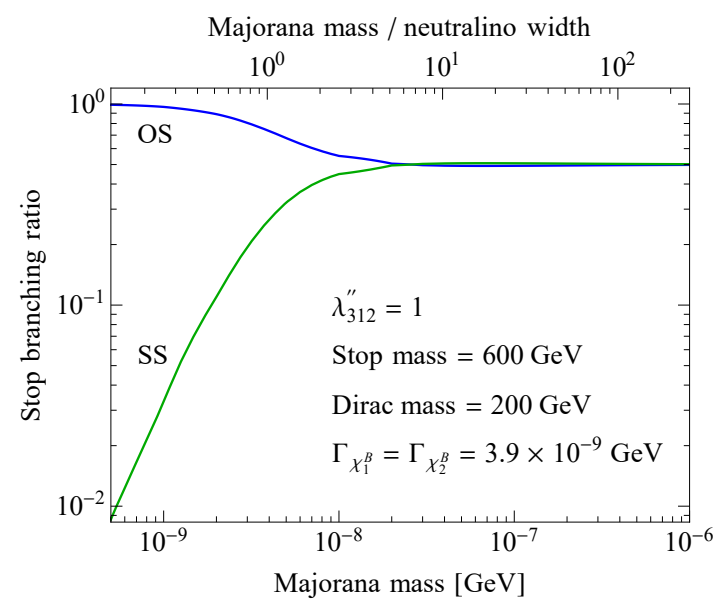

(b)

Figure 6.8: Partial decay widths (a) and branching ratios (b) for $600 \mathrm{GeV}$ stops decaying into opposite sign (OS) and same sign (SS) tops through approximately 200 $\mathrm{GeV}$ bino neutralinos with $\lambda_{312}^{\prime \prime}=1$.

duced at the LHC and that it decays into a top and a neutralino. Then, for Dirac neutralinos, the final state quarks will always be $\bar{t} t d_{j} d_{k}$ while, for Majorana neutralinos, the final state quarks can either be $\bar{t} t d_{j} d_{k}$ or $\overline{t t} \bar{d}_{j} \bar{d}_{k}$. Resonant stop production with Majorana neutralinos can lead to same sign tops whereas opposite sign tops are always produced for Dirac neutralinos. Same sign tops can potentially lead to same sign leptons, which is a powerful phenomenological signature for separating signal from background. In contrast, a final state of $\bar{t} t d_{j} d_{k}$ is difficult to distinguish from a background such as $t \bar{t}$ and jets. Similarly, stop pair production is also affected by whether or not the neutralino is Dirac or Majorana. If both stops decay into neutralinos, then a total of four tops will be produced. Dirac neutralinos will always result in two positively and two negatively charged tops. However, Majorana neutralinos will result in two positively and two negatively charged tops only half of the time. For the other half, three tops with the same sign will be produced, along with a single top with the opposite sign. Of note, the latter case has a larger probability of producing a same sign lepton pair. 
There is one more possible decay mode that we need to consider. If the Majorana mass term is large enough so that the mass splitting between the pseudo-Dirac neutralino states is non-negligible, then the decays $\chi_{2}^{B} \rightarrow \chi_{1}^{B} Z$ and $\chi_{2}^{B} \rightarrow \chi_{1}^{B} h$ potentially open up. In these decays the $Z$ and $h$ are off-shell for small mass splittings. However, for all neutralino masses and $\lambda_{3 j k}^{\prime \prime}$ couplings considered in this analysis, the decay width for the neutralinos is relatively small (generally less than $1 \mathrm{GeV}$ ). As a result, only a modest Majorana mass term is needed to ensure that opposite sign and same sign tops are produced equally from stop decays. Thus, we make the following assumption. If the $\mathrm{U}(1)_{R}$ symmetry is broken, then the Majorana mass term is large enough such that the stops decay into opposite sign and same sign tops with equal branching ratios, while, at the same time, is small enough so that the decays $\chi_{2}^{B} \rightarrow \chi_{1}^{B} Z$ and $\chi_{2}^{B} \rightarrow \chi_{1}^{B} h$ can be safely ignored. This also has the added benefit of making the analysis of the possible decay chains simpler. Finally, we also note that under this assumption, the phenomenology of the MRSSM with a broken $\mathrm{U}(1)_{R}$ symmetry is essentially identical to the RPVMSSM.

Now that we have discussed the various decay modes for the stop, neutralino, and chargino, we consider all processes involving stop production. First, consider the case were the $\mathrm{U}(1)_{R}$ symmetry is strictly preserved. Then, enumerating all the possibilities, we get the following list

(1)

$$
\begin{aligned}
p p \rightarrow \tilde{t}^{*} & \rightarrow d_{j} d_{k} \\
p p & \rightarrow \tilde{t}^{*} \rightarrow \bar{b} \chi^{-} \rightarrow \bar{b} b d_{j} d_{k} \\
p p \rightarrow \tilde{t}^{*} \tilde{t} & \rightarrow \bar{t} \chi^{0} t \bar{\chi}^{0} \\
& \rightarrow \bar{t} t d_{j} d_{k} t \bar{t} \bar{d}_{j} \bar{d}_{k}
\end{aligned}
$$

$$
\begin{aligned}
p p & \rightarrow \tilde{t}^{*} \rightarrow \bar{t} \chi^{0} \rightarrow \bar{t} t d_{j} d_{k} \\
p p & \rightarrow \tilde{t}^{*} \tilde{t} \\
& \rightarrow d_{j} d_{k} \bar{d}_{j} \bar{d}_{k} \\
p p & \rightarrow \tilde{t}^{*} \tilde{t} \rightarrow \bar{b} \chi^{-} b \chi^{+} \\
& \rightarrow \bar{b} b d_{j} d_{k} b \bar{b} \bar{d}_{j} \bar{d}_{k}
\end{aligned}
$$




$$
\begin{aligned}
p p \rightarrow \tilde{t}^{*} \tilde{t} & \rightarrow d_{j} d_{k} t \bar{\chi}^{0} \quad \text { (8) } \begin{aligned}
p p \rightarrow \tilde{t}^{*} \tilde{t} & \rightarrow d_{j} d_{k} b \chi^{+} \\
& \rightarrow d_{j} d_{k} t \bar{t} \bar{d}_{j} \bar{d}_{k}
\end{aligned} \quad \rightarrow d_{j} d_{k} b \bar{b} \bar{d}_{j} \bar{d}_{k} \\
p p \rightarrow \tilde{t}^{*} \tilde{t} & \rightarrow \bar{t} \chi^{0} b \chi^{+} \\
& \rightarrow \bar{t} t d_{j} d_{k} b \bar{b} \bar{d}_{j} \bar{d}_{k} .
\end{aligned}
$$

If, instead, the $\mathrm{U}(1)_{R}$ symmetry is broken, then processes $2,5,7$, and 9 need to be modified

(2) $p p \rightarrow \tilde{t}^{*} \rightarrow \bar{t} \chi^{0} \rightarrow\left\{\begin{array}{l}\bar{t} t d_{j} d_{k} \\ \overline{t t} \bar{d}_{j} \bar{d}_{k}\end{array}\right.$

$$
p p \rightarrow \tilde{t}^{*} \tilde{t} \rightarrow d_{j} d_{k} t \chi^{0} \rightarrow\left\{\begin{array}{l}
d_{j} d_{k} t \bar{t} \bar{d}_{j} \bar{d}_{k} \\
d_{j} d_{k} t t d_{j} d_{k}
\end{array}\right.
$$

$$
p p \rightarrow \tilde{t}^{*} \tilde{t} \rightarrow \bar{t} \chi^{0} t \chi^{0} \rightarrow\left\{\begin{array}{l}
\bar{t} t d_{j} d_{k} t \bar{t} \bar{d}_{j} \bar{d}_{k} \\
\bar{t} t d_{j} d_{k} t t d_{j} d_{k} \\
\overline{t t} \bar{d}_{j} \bar{d}_{k} t \bar{t} \bar{d}_{j} \bar{d}_{k} \\
\bar{t} \bar{t} \bar{d}_{j} \bar{d}_{k} t t d_{j} d_{k}
\end{array}\right.
$$

(9) $p p \rightarrow \tilde{t}^{*} \tilde{t} \rightarrow \bar{t} \chi^{0} b \chi^{+} \rightarrow\left\{\begin{array}{l}\bar{t} t d_{j} d_{k} b \bar{b} \bar{d}_{j} \bar{d}_{k} \\ \overline{t t} \bar{d}_{j} \bar{d}_{k} b \bar{b} \bar{d}_{j} \bar{d}_{k} .\end{array}\right.$

Where appropriate, each process also includes its charge conjugated version.

Processes 1 and 4 can be constrained by using the results for stop LSP (section 6.3.1.2) with appropriate modifications to the branching ratios. When presenting plots of the parameter space, the region ruled out by process 1 is referred to as the region excluded by dijets searches. Likewise, the region ruled out by process 4 is referred to as the region excluded by paired dijet searches. Further exclusion is possible if other types of experimental searches are considered. Our methodology for choosing which searches to recast is as follows. First, there have been several experimental searches featuring supersymmetric particles decaying through the $\lambda_{3 j k}^{\prime \prime}$ couplings. We select three of the most recent searches of this variety. These are 
[214-216], of which all are from ATLAS. Next, notice that many of the different possible final states contain either four tops or two same sign tops. We therefore examine searches that constrain these types of final states. This led us to choosing searches $[217,218]$ from ATLAS and [219] from CMS. A brief outline of the strategy for each search is summarized in table 6.2. The region of parameter space ruled out by these searches is referred to as the region excluded by neutralino LSP searches.

The procedure used to recast these searches is similar to the procedures used to recast dijets searches described above. First, the neutralino mass is set to 200 $\mathrm{GeV}$ and the stop mass is scanned between 200 and $1000 \mathrm{GeV}$. For all combinations, we use MadGraph, Pythia, and Delphes to simulate 10000 events for each of the nine possible decay chains. This was done twice for processes $2,5,7$, and 9 , once with the $\mathrm{U}(1)_{R}$ symmetry preserved and a second time with it broken. Code was implemented to simulate the cuts for each of the six searches. We verified our code by reproducing each search with good accuracy. Using the simulated events, our code produced acceptances for every signal region described within each search. Then, within the stop mass and $\lambda_{3 j k}^{\prime \prime}$ parameter space, the acceptances are combined with production cross sections and appropriate branching ratios to determine the number of expected signals for each signal region. The 95\% CL upper limit for each signal region were then determined. Searches [214-216] explicitly provided these upper limits. Conversely, searches [217-219] did not, and so we calculate the upper limits using the $\mathrm{CL}_{\mathrm{S}}$ technique $[85,86]$. A point in parameter space is then excluded if the expected number of signals in any of the signal regions exceed its upper limit.

The region of small $\lambda_{3 j k}^{\prime \prime}$ can additionally be constrained by searches for displaced vertices. The efficiency for reconstructing a single displaced vertex is to good approximation only a function of the mass $m$ and decay length $c \tau$ of the particle in- 


\begin{tabular}{|c|c|c|}
\hline Collaboration & Search & Strategy \\
\hline ATLAS & [214] & $\begin{array}{c}2 \text { (potentially negative) same sign leptons, } \\
\text { total number of leptons, } \\
\text { jets with } p_{T}>25,40, \text { or } 50 \mathrm{GeV}, \text { b-jets, } \\
\text { MET, } m_{\text {eff }}=\sum_{\text {jets }} p_{T}+\mathrm{MET} \\
\text { leptons }\end{array}$ \\
\hline ATLAS & {$[215]$} & $\begin{array}{c}\text { large }(R=1.0) \text { jets } J_{i} \\
p_{T J_{1}}>440 \mathrm{GeV},\left|\Delta \eta_{J_{1} J_{2}}\right|<1.4 \\
M_{J}^{\Sigma}=\sum_{i=1}^{4} m_{J_{i}}, \text { small }(R=0.4) \text { b-jets }\end{array}$ \\
\hline ATLAS & {$[216]$} & $\begin{array}{c}\text { at least } 1 \text { lepton, } \\
\text { jets with } p_{T}>40 \text { or } 60 \mathrm{GeV} \\
\text { b-jets with } p_{T}>40 \text { or } 60 \mathrm{GeV}\end{array}$ \\
\hline ATLAS & [217] & $\begin{array}{c}\text { exactly } 1 \text { lepton, jets, b-jets, } \\
\text { mass-tagged jets }=\text { large }(R=1.0) \text { jets with } \\
\text { cuts, } \\
m_{b b}^{\min \Delta R}=\text { invariant mass of closest b-jets, } \\
\text { MET, MET }+M_{T}(\ell, \text { MET }) \\
\text { where } M_{T}=\text { transverse mass }\end{array}$ \\
\hline ATLAS & [218] & $\begin{array}{c}2 \text { same sign leptons, jets, b-jets, } \\
\qquad \mathrm{MET}, H_{T}=\sum_{\substack{\text { jets } \\
\text { leptons }}} p_{T}\end{array}$ \\
\hline CMS & {$[219]$} & $\begin{array}{c}2 \text { same sign leptons, jets, b-jets, } \\
M_{T}^{\min }=\min \left(M_{T}\left(\ell_{1}, \mathrm{MET}\right), M_{T}\left(\ell_{2}, \mathrm{MET}\right)\right) \\
\text { where } M_{T}=\text { transverse mass, } \\
\operatorname{MET}, H_{T}=\sum_{\text {jets }} p_{T}\end{array}$ \\
\hline
\end{tabular}

Table 6.2: Neutralino LSP searches. For searches [215,217], we use FastJet [77,220] for manipulating large $(R=1)$ jets. This mainly involves jet reclustering and jet trimming. Additionally, searches that feature missing transverse energy (MET) either have very lenient cuts on this quantity or also contain signal regions probing $R$-parity conserving supersymmetry signatures.

volved. We make the further approximation that this function, which we call $f(m, c \tau)$, can be factorized as $f_{1}(m) f_{2}(c \tau)$. This is justified by the results of Ref. [221], which presents upper limits on the cross section for pair production of hadronically decaying neutralinos as a function of their mass for a fixed decay length. These results 
are based on the CMS search [222]. The function $f_{1}(m)$ can be read from Ref. [221] up to a multiplicative factor that can be absorbed in $f_{2}(c \tau)$. The latter function can be extracted from Ref. [159], which presents exclusion limits on displaced vertices for Higgsino LSP in the parameter space of Higgsino mass and decay length. These results are based on the same search and assume charged Higgsinos decay promptly to the almost degenerate neutral one. Higgsinos are again assumed to be pair produced and for the lightest one to decay hadronically. Knowing the cross section involved and upper limit on the signal, $f_{2}(c \tau)$ can be reconstructed everywhere except for very short and very long decay lengths. In these regions, the efficiency decreases exponentially as expected and we extrapolate this behaviour. This allows for a complete reconstruction of $f(m, c \tau)$, which is shown in figure 6.9.

Next, note that the displaced vertices in our model result from neutralino decays. We consider both neutralino pair production and neutralinos produced from stop decays. Furthermore, in this part of the parameter space, we assume the Higgsino-up charginos decay dominantly into neutralinos. This is in contrast to our previous benchmark points where the RPV decay for charginos was assumed. For small values of $\lambda_{3 j k}^{\prime \prime}$ the charginos will decay into neutralinos provided that the spectrum is not too degenerate. A large enough splitting can easily be generated provided that the wino is not exceptionally heavy. To compute the cross sections for Higgsinoup and bino pair production we use Prospino. The bino cross section depends on the masses for the first and second generations of squarks. We consider two different cases. For the first case, we decouple the squarks by setting their masses to $10 \mathrm{TeV}$. For the second case, we set their masses to $1 \mathrm{TeV}$. Combining these cross sections with the known $f(m, c \tau)$, limits on displaced vertices can easily be applied to our parameter spaces using once again Ref. [222]. 


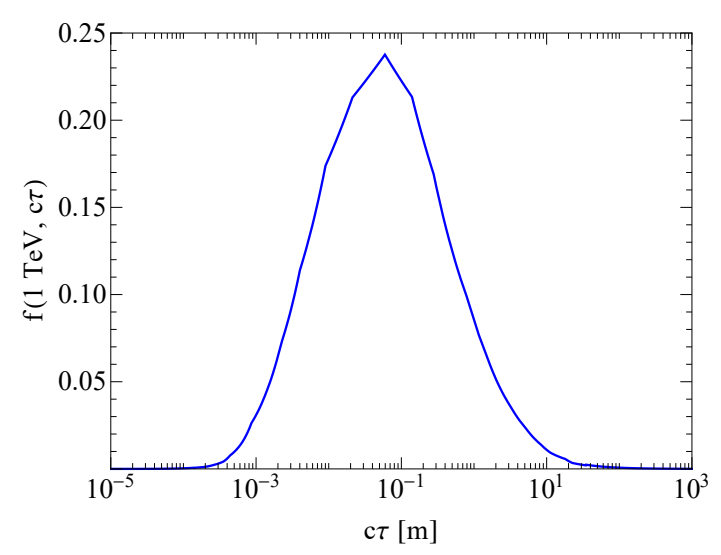

(a)

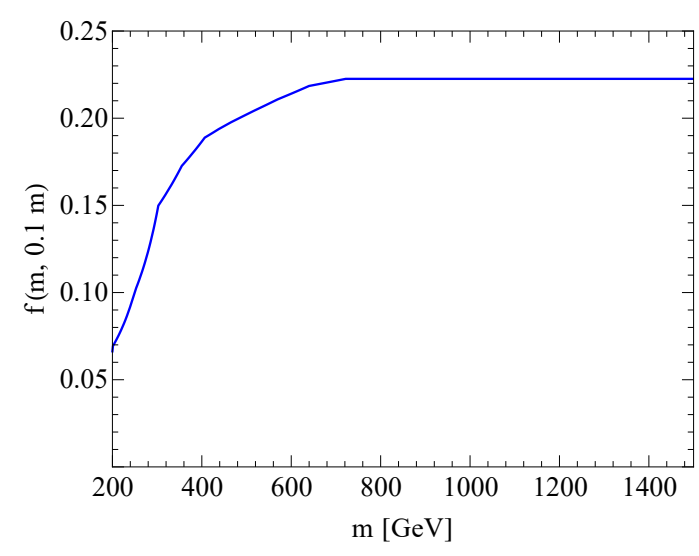

(b)

Figure 6.9: The efficiency for reconstructing a single vertex $f(m, c \tau)$ for (a) fixed $m=1 \mathrm{TeV}$ and (b) fixed $c \tau=0.1 \mathrm{~m}$ for the CMS search [222].

Combining all types of constraints discussed above, we present exclusion plots within the stop mass and $\lambda_{3 j k}^{\prime \prime}$ parameter space. Figures 6.10 and 6.11 show the regions excluded provided that the $\mathrm{U}(1)_{R}$ symmetry is strictly preserved for bino LSP and Higgsino-up LSP, respectively. Similarly, figures 6.12 and 6.13 show the regions excluded when the $\mathrm{U}(1)_{R}$ symmetry is broken, again for bino LSP and Higgsino-up LSP, respectively. Notice that the limits coming from the neutralino LSP searches (green area) do not extend into the smallest values of $\lambda_{3 j k}^{\prime \prime}$ shown in the plots. These searches rely on promptly decaying particles and so we conservatively cutoff their exclusion capabilities when the neutralino's decay length becomes longer than $1 \mathrm{~mm}$ [159]. The green area excluded for $\lambda_{3 j k}^{\prime \prime} \lesssim 0.1$ mostly results from stop pair production with subsequent decay into neutralinos. Larger stop masses are excluded for the bino than the Higgsino-up for this range of $\lambda_{3 j k}^{\prime \prime}$ coupling because there is no competing chargino decay. The green area for $\lambda_{3 j k}^{\prime \prime} \gtrsim 0.1$ is mostly excluded by resonant stop production with subsequent decay through neutralinos. Note that quite a bit more of this parameter space is excluded when the $\mathrm{U}(1)_{R}$ symmetry is broken. This is largely 
due to the production of same sign tops which is absent when the $\mathrm{U}(1)_{R}$ symmetry is preserved. Another interesting feature for this part of the parameter space is that approximately equal areas are excluded for $\lambda_{312}^{\prime \prime}$ and $\lambda_{313}^{\prime \prime}$. The reason for this is that while the cross section for resonant stop production is smaller for $\lambda_{313}^{\prime \prime}$, the efficiencies are generally larger than for $\lambda_{312}^{\prime \prime}$ due to the production of extra bottom quarks. These two effects are seen to approximately compensate one another.

Figures 6.12 and 6.13 , with the $\mathrm{U}(1)_{R}$ symmetry broken, are similar to figures shown in Ref. [131]. (The figures within Ref. [131] are presented within a RPVMSSM context. However, as previously noted, the phenomenology of the MRSSM with the $\mathrm{U}(1)_{R}$ symmetry broken is nearly identical to the RPVMSSM.) Our neutralino LSP searches exclude larger amounts of the parameter space than the corresponding searches considered by Ref. [131]. This is simply because we use more recent, and, in particular, $13 \mathrm{TeV}$ experimental searches. Our exclusion regions for displaced vertices searches are, on the other hand, significantly smaller than Ref. [131].

\subsubsection{Placing limits on first and second generation squarks}

\subsubsection{Squark production}

As previously mentioned, the label squarks refers to only the first and second gener-

ations. Explicitly, we consider the states $\tilde{d}_{L}, \tilde{u}_{L}, \tilde{s}_{L}, \tilde{c}_{L}, \tilde{d}_{R}, \tilde{u}_{R}, \tilde{s}_{R}$, and $\tilde{c}_{R}$ and their charge conjugates. The squarks are taken to be mass degenerate.

As we are considering the $\lambda_{3 j k}^{\prime \prime}$ couplings, squarks can only be produced in pairs. In general, squark pairs are produced either from initial state gluons or initial state quarks with a t-channel gluino propagator. (We again ignore potential squark production involving two $\lambda_{3 j k}^{\prime \prime}$ couplings.) Although, due to the Dirac nature of the gluino, some of the production mechanisms present in the MSSM are forbidden within the 

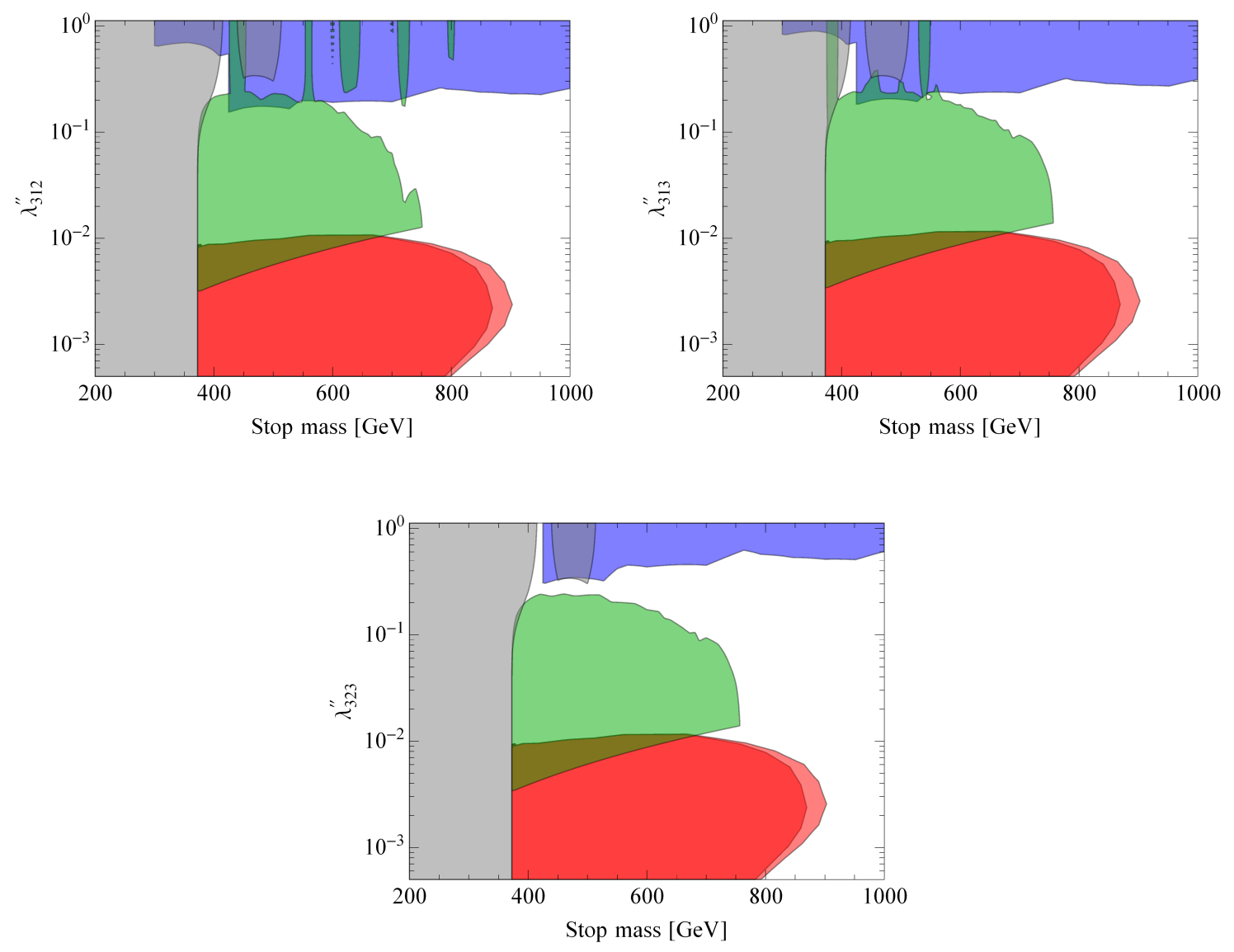

Figure 6.10: Exclusion plots for a $200 \mathrm{GeV}$ bino neutralino LSP with the $\mathrm{U}(1)_{R}$ symmetry strictly preserved. The grey region on the left side of the plots $\left(m_{\tilde{t}} \lesssim 375\right.$ $\mathrm{GeV}$ ) is excluded by paired dijet searches. Next, consider the middle region of the plots. Starting from large $\lambda_{3 j k}^{\prime \prime}$ couplings and working downwards, the blue region is excluded by dijet searches, the green region is excluded by neutralino LSP searches, and the red regions are excluded by displaced vertices searches. Bino pair production, which contributes to the displaced vertices limits, depends on the masses of the first and second generations of squarks. Setting the masses of these squarks to $10 \mathrm{TeV}$ results in the darker red region being excluded. Instead, setting the masses of these squarks to $1 \mathrm{TeV}$ excludes both the darker red and lighter red regions.

MRSSM. In particular, diagrams which require a gluino Majorana mass insertion are forbidden $[182,183]$. This prevents the production of $\tilde{q}_{L} \tilde{q}_{L}, \tilde{q}_{R} \tilde{q}_{R}$, and $\tilde{q}_{L} \tilde{q}_{R}^{*}$ and their charge conjugates. Additionally, breaking the $\mathrm{U}(1)_{R}$ symmetry with a small gluino mass will only reintroduce the forbidden diagrams by negligible amounts. There is no 

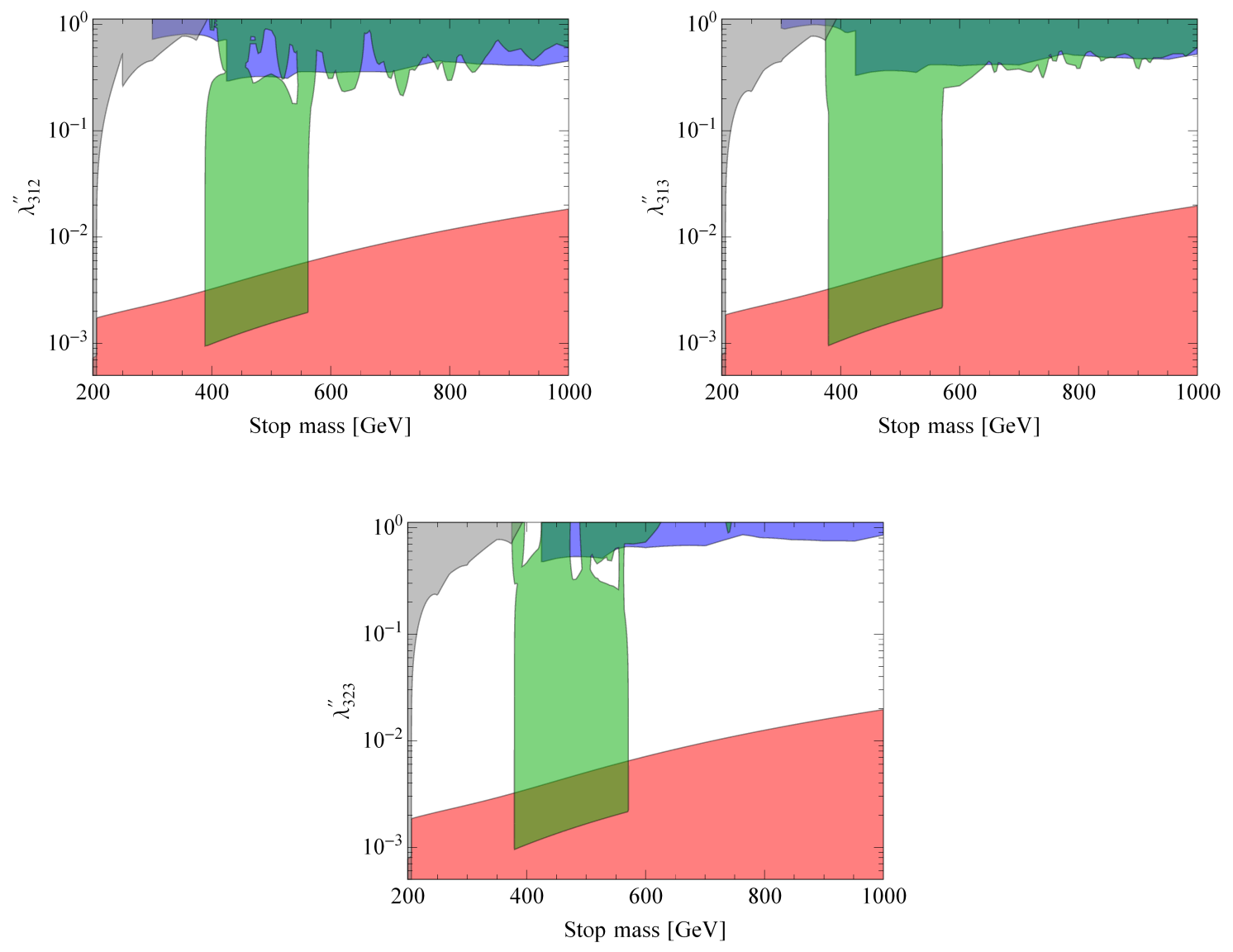

Figure 6.11: Exclusion plots for a $200 \mathrm{GeV}$ Higgsino-up neutralino LSP with the $\mathrm{U}(1)_{R}$ symmetry strictly preserved. The grey region on the left side of the plots $\left(m_{\tilde{t}} \lesssim 205 \mathrm{GeV}\right)$ is excluded by paired dijet searches. Next, consider the middle region of the plots. Starting from large $\lambda_{3 j k}^{\prime \prime}$ couplings and working downwards, the blue region is excluded by dijet searches, the green region is excluded by neutralino LSP searches, and the red region is excluded by displaced vertices searches.

enhancement comparable to stops decaying into same sign tops. As noted above, this enhancement requires the neutralino from the stop decay to be produced on-shell. Here, the four-momentum of the t-channel gluino is spacelike and thus the gluino is never on-shell.

As a result, we are interested in the production of $\tilde{q}_{L} \tilde{q}_{L}^{*}, \tilde{q}_{R} \tilde{q}_{R}^{*}, \tilde{q}_{L} \tilde{q}_{R}$, and $\tilde{q}_{L}^{*} \tilde{q}_{R}^{*}$. We use MadGraph to calculate the LO cross sections for these final states. To estimate 

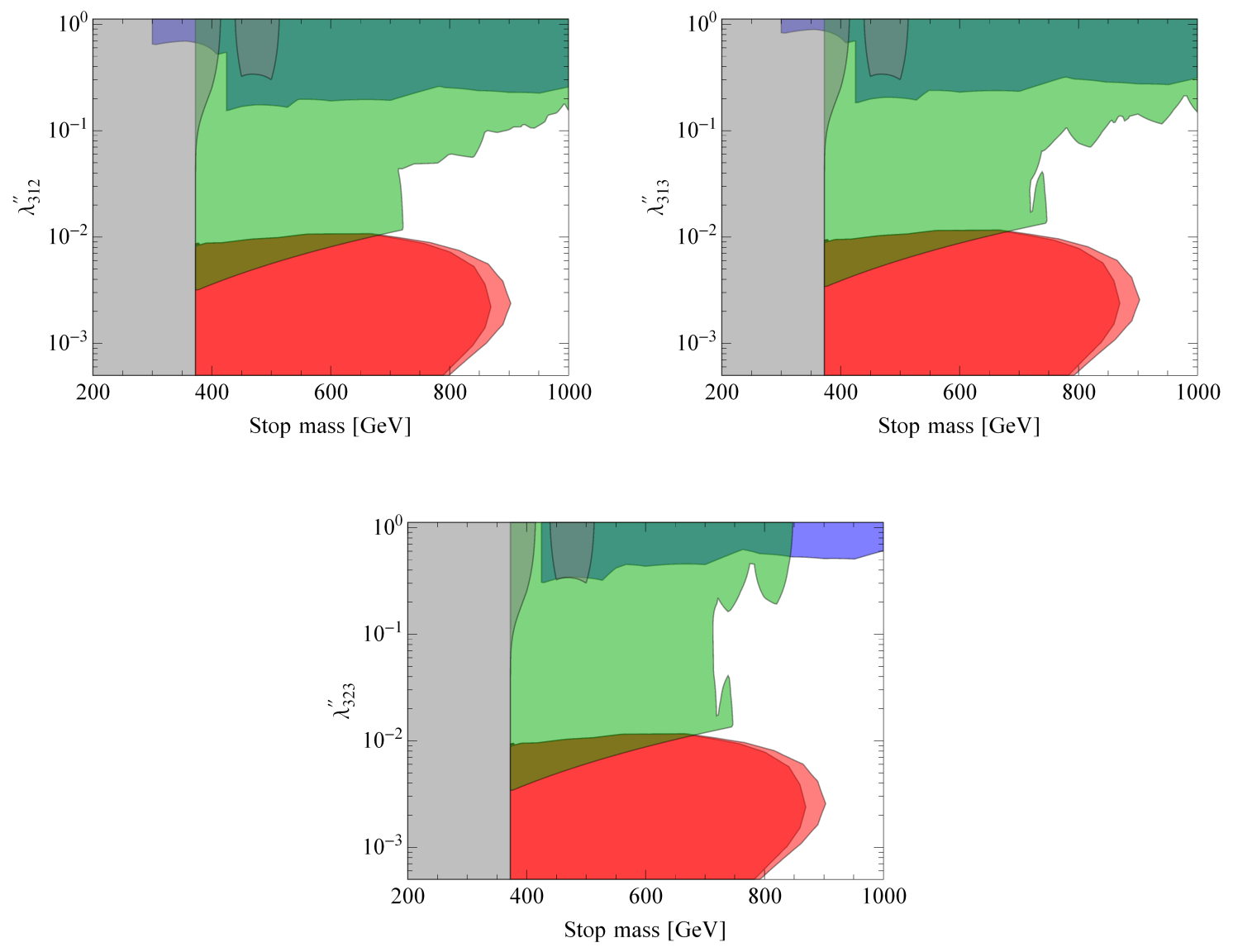

Figure 6.12: Exclusion plots for a $200 \mathrm{GeV}$ bino neutralino LSP with the $\mathrm{U}(1)_{R}$ symmetry broken. The grey region on the left side of the plots $\left(m_{\tilde{t}} \lesssim 375 \mathrm{GeV}\right)$ is excluded by paired dijet searches. Next, consider the middle region of the plots. Starting from large $\lambda_{3 j k}^{\prime \prime}$ couplings and working downwards, the blue region is excluded by dijet searches, the green region is excluded by neutralino LSP searches, and the red regions are excluded by displaced vertices searches. Bino pair production, which contributes to the displaced vertices limits, depends on the masses of the first and second generations of squarks. Setting the masses of these squarks to $10 \mathrm{TeV}$ results in the darker red region being excluded. Instead, setting the masses of these squarks to $1 \mathrm{TeV}$ excludes both the darker red and lighter red regions.

higher order effects, we use NNLL-fast [79-82, 201,223-225] to compute MSSM Kfactors for squark-antisquark and squark-squark production as a function of the mass of the squarks. The gluino mass is set to $2 \mathrm{TeV}$ for both steps. Below, we present plots using both the LO and the MSSM K-factor improved cross sections. 

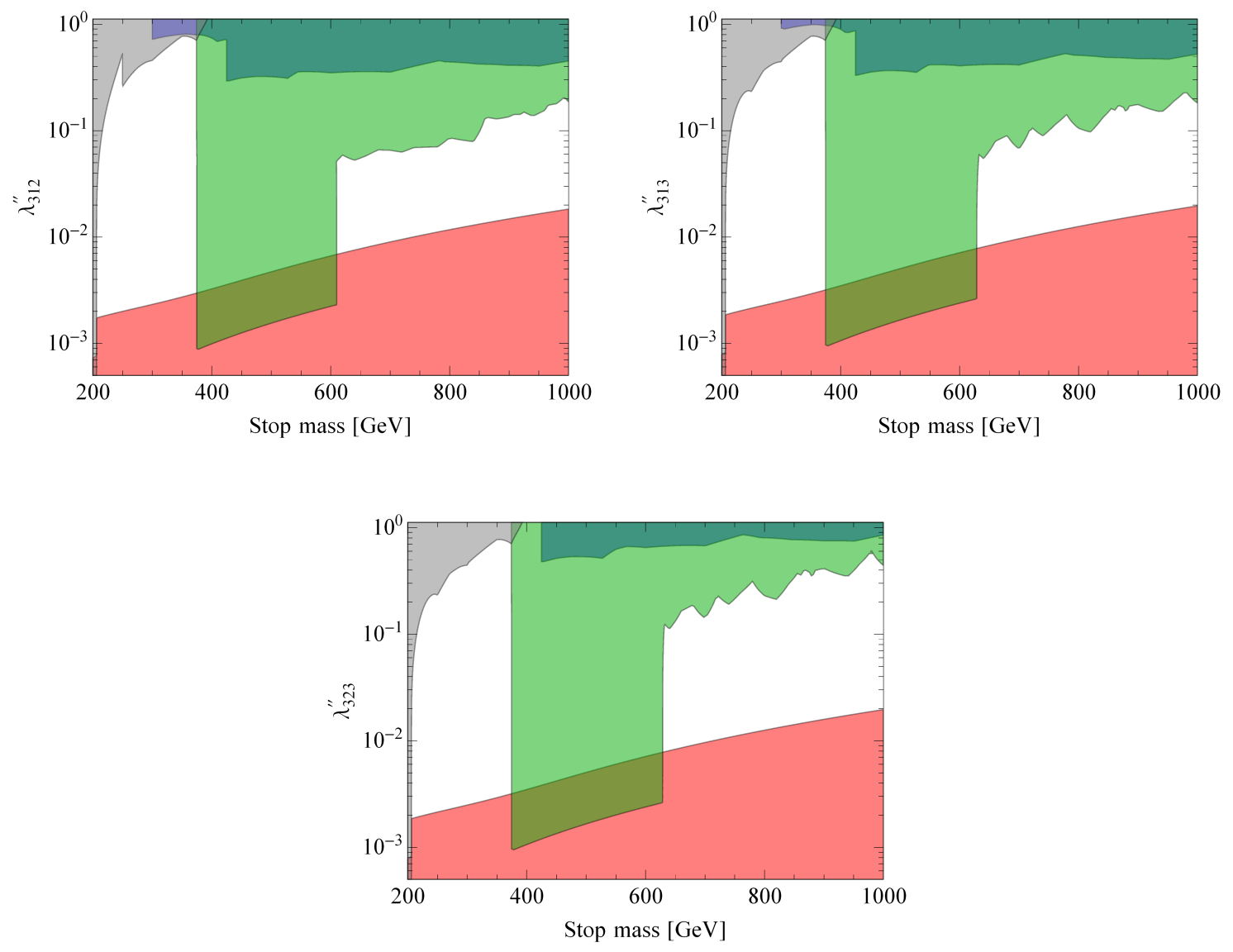

Figure 6.13: Exclusion plots for a $200 \mathrm{GeV}$ Higgsino-up neutralino LSP with the $\mathrm{U}(1)_{R}$ symmetry broken. The grey region on the left side of the plots $\left(m_{\tilde{t}} \lesssim 205\right.$ $\mathrm{GeV}$ ) is excluded by paired dijet searches. Next, consider the middle region of the plots. Starting from large $\lambda_{3 j k}^{\prime \prime}$ couplings and working downwards, the blue region is excluded by dijet searches, the green region is excluded by neutralino LSP searches, and the red region is excluded by displaced vertices searches.

\subsubsection{Neutralino LSP}

To avoid long lived squarks, we require a neutralino LSP. We again only consider a bino neutralino or a Higgsino-up neutralino. Furthermore, we consider the stop heavier than the squarks but light enough so that the neutralinos and charginos 
decay promptly. Then, the possible decay chains are

(1)

$$
\begin{aligned}
p p & \rightarrow \tilde{q}^{*} \tilde{q} \\
& \rightarrow d_{j / k} t \bar{d}_{j / k} \bar{t}
\end{aligned}
$$

$$
\begin{aligned}
p p \rightarrow \tilde{q}^{*} \tilde{q} & \rightarrow \bar{q}^{\prime} \chi^{+} q^{\prime} \chi^{-} \\
& \rightarrow \bar{q}^{\prime} \bar{b} \bar{d}_{j} \bar{d}_{k} q^{\prime} b d_{j} d_{k}
\end{aligned}
$$

$$
\begin{aligned}
p p \rightarrow \tilde{q}^{*} \tilde{q} & \rightarrow d_{j / k} t q^{\prime} \chi^{-} \\
& \rightarrow d_{j / k} t q^{\prime} b d_{j} d_{k}
\end{aligned}
$$

$$
\begin{aligned}
p p \rightarrow \tilde{q}^{*} \tilde{q} & \rightarrow \bar{q} \chi^{0} q \bar{\chi}^{0} \\
& \rightarrow \bar{q} t d_{j} d_{k} q \bar{t} \bar{d}_{j} \bar{d}_{k} \\
p p \rightarrow \tilde{q}^{*} \tilde{q} & \rightarrow d_{j / k} t q \bar{\chi}^{0} \\
& \rightarrow d_{j / k} t q \bar{t} \bar{d}_{j} \bar{d}_{k} \\
p p \rightarrow \tilde{q}^{*} \tilde{q} & \rightarrow \bar{q} \chi^{0} q^{\prime} \chi^{-} \\
& \rightarrow \bar{q} t d_{j} d_{k} q^{\prime} b d_{j} d_{k} .
\end{aligned}
$$

The decays involving the $\lambda_{3 j k}^{\prime \prime}$ coupling can only occur for squarks $\tilde{d}_{R}$ and $\tilde{s}_{R}$ and their charge conjugates. The notation $d_{j / k}$ stands for $d_{j}$ or $d_{k}$. Whether the final state is $d_{j}$ or $d_{k}$ depends on which squark is decaying and which one of the $\lambda_{3 j k}^{\prime \prime}$ is non-zero. The other squarks are required to decay through either a neutralino or chargino. If, instead, the $\mathrm{U}(1)_{R}$ symmetry is broken, then processes 2,4 , and 6 are modified
(2) $p p \rightarrow \tilde{q}^{*} \tilde{q} \rightarrow \bar{q} \chi^{0} q \chi^{0} \rightarrow\left\{\begin{array}{l}\bar{q} t d_{j} d_{k} q \bar{t} \bar{d}_{j} \bar{d}_{k} \\ \bar{q} t d_{j} d_{k} q t d_{j} d_{k} \\ \bar{q} \bar{t} \bar{d}_{j} \bar{d}_{k} q \bar{t} \bar{d}_{j} \bar{d}_{k} \\ \bar{q} \bar{t} \bar{d}_{j} \bar{d}_{k} q t d_{j} d_{k}\end{array}\right.$
(4) $p p \rightarrow \tilde{q}^{*} \tilde{q} \rightarrow d_{j / k} t q \chi^{0} \rightarrow\left\{\begin{array}{l}d_{j / k} t q \bar{t} \bar{d}_{j} \bar{d}_{k} \\ d_{j / k} t q t d_{j} d_{k}\end{array}\right.$
(6) $p p \rightarrow \tilde{q}^{*} \tilde{q} \rightarrow \bar{q} \chi^{0} q^{\prime} \chi^{-} \rightarrow\left\{\begin{array}{l}\bar{q} t d_{j} d_{k} q^{\prime} b d_{j} d_{k} \\ \bar{q} \bar{t} \bar{d}_{j} \bar{d}_{k} q^{\prime} b d_{j} d_{k}\end{array}\right.$ 
Note that these are very similar to the decay chains for stop pair production. Although, a major difference is that at most two tops are produced whereas stop pair production resulted in four tops. If the $\mathrm{U}(1)_{R}$ symmetry is broken, then production of two same sign leptons can still potentially take place, but now with a much lower probability than in the stop scenario.

A nearly identical procedure to the one described above is used to constrain the parameter space. Here, we scan the stop mass and neutralino mass parameter space simulating each of the decay chains above. The acceptances are once again determined for the neutralino LSP searches of table 6.2. We then compute the branching ratios for each of the squarks. Combining the cross sections, branching ratios, and acceptances, we produce exclusion curves within the stop mass and neutralino mass parameter space.

Figures 6.14 and 6.15 present the exclusions curves for bino and Higgsino-up LSP, respectively. For each plot, the $\lambda_{3 j k}^{\prime \prime}$ have been set to one. Note that curves are shown only for the case in which the $\mathrm{U}(1)_{R}$ symmetry is preserved. As previously noted, breaking the symmetry only introduces a small probability of producing a same sign lepton pair. Consequently, the searches that require same sign leptons are less constraining then the searches that require multiple jets. The ATLAS search [216], which does not rely on a same sign lepton pair, dominates for the entirety of the exclusion curve for both the $\mathrm{U}(1)_{R}$ symmetry preserved and broken. As a result, the exclusion curves for the two cases are the same. Additionally, the cases $\lambda_{313}^{\prime \prime}$ and $\lambda_{323}^{\prime \prime}$ are presented together. The only difference between these two cases is the production of down versus strange quarks, which is irrelevant to the searches involved. An interesting feature is that the excluded region prefers large neutralino masses. This follows from the decay chains as most of the final state quarks come from decaying 


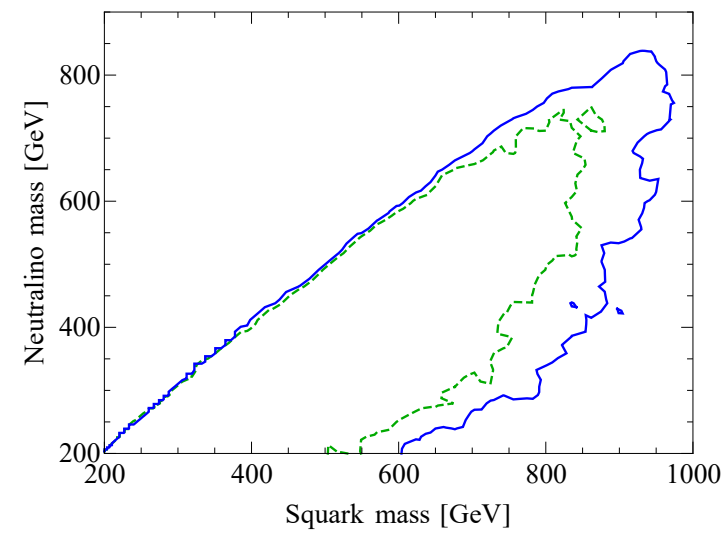

(a) $\lambda_{312}^{\prime \prime}=1$

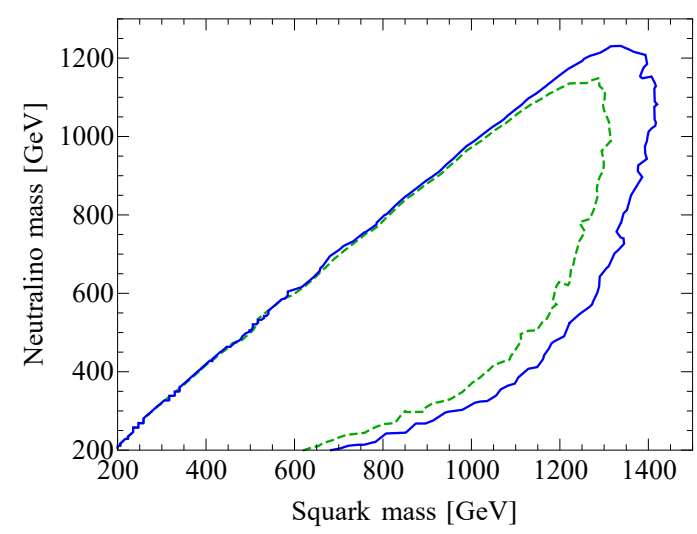

(b) $\lambda_{313}^{\prime \prime}=1$ or $\lambda_{323}^{\prime \prime}=1$

Figure 6.14: Exclusion curves within the neutralino-squark mass parameter space for a bino neutralino. Figure (a) presents $\lambda_{312}^{\prime \prime}=1$ while (b) presents either $\lambda_{313}^{\prime \prime}=1$ or $\lambda_{323}^{\prime \prime}=1$. The dashed green curves assume leading order squark production whereas the solid blue curves include MSSM K-factors.

neutralinos or charginos. This is in contrast to RPC MSSM searches with decaying squarks, which exclude light neutralino masses preferentially (see figure 11(a) of the ATLAS search [226] for example). Finally, these limits on squark production are presented in a MRSSM framework. However, the exclusion curves can also be seen as lower limits for the RPVMSSM as the major difference is the exclusion of some of the possible production cross sections.

\subsection{Conclusion}

In this chapter, we studied the LHC phenomenology of a supersymmetric model with a $\mathrm{U}(1)_{R}$ symmetry which is identified with baryon number. The model we considered is an extension of the MRSSM with the inclusion of an $R$-parity breaking term of

the form $\lambda_{i j k}^{\prime \prime} U_{i}^{c} D_{j}^{c} D_{k}^{c}$. Because of the non-standard baryon number assignment of the superpartners, such a term is baryon number conserving in this model. This relaxes the bounds on the $\lambda^{\prime \prime}$ couplings significantly compared to the RPVMSSM. In 


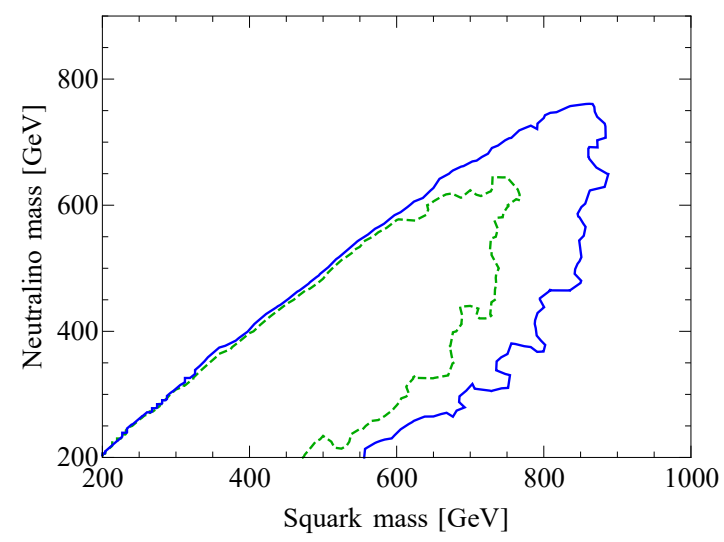

(a) $\lambda_{312}^{\prime \prime}=1$

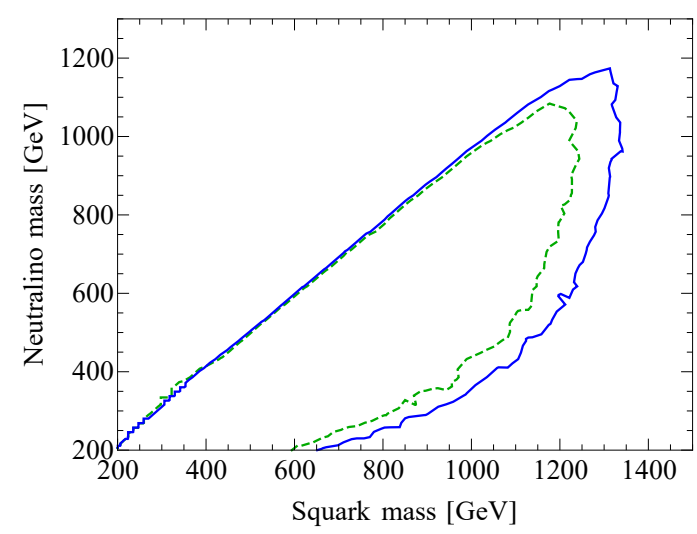

(b) $\lambda_{313}^{\prime \prime}=1$ or $\lambda_{323}^{\prime \prime}=1$

Figure 6.15: Exclusion curves within the neutralino-squark mass parameter space for a Higgsino-up neutralino. Figure (a) presents $\lambda_{312}^{\prime \prime}=1$ while (b) presents either $\lambda_{313}^{\prime \prime}=1$ or $\lambda_{323}^{\prime \prime}=1$. The dashed green curves assume leading order squark production whereas the solid blue curves include MSSM K-factors.

particular, the bounds from neutron-antineutron oscillation and from double nucleon decay are considerably loosened. However, they cannot be removed completely as the $\mathrm{U}(1)_{R}$ will be broken by the gravitino mass and communicated to the superpartners of the Standard Model by anomaly mediation. Furthermore, the gravitino must be heavier than the proton to avoid proton decay to a gravitino and a kaon. Flavour physics also puts bounds on products of $\lambda^{\prime \prime}$ couplings which are the same in our model as in the RPVMSSM.

The introduction of large $\lambda^{\prime \prime}$ couplings leads to a collider phenomenology that is significantly different from the MSSM and from the RPVMSSM with very small $\lambda^{\prime \prime}$ couplings. We examined simplified models where a single one of the $\lambda^{\prime \prime}$ involving the third generation is large. We looked at both single and pair production of stops and their subsequent decays for bino or Higgsino-up LSP. When Majorana mass terms are included, a Dirac neutralino splits into two states close in mass. We showed that when this mass splitting is larger than the width of the neutralino, the stop can decay via 
this neutralino to two same sign tops. On the other hand, when the mass splitting is smaller, the branching ratio of a stop decaying to two same sign tops is highly suppressed. Because same sign leptons are a powerful tool to reject background, the two cases present different phenomenology and in this chapter we presented results for both hypotheses. We also presented limits on the masses of the first and second generation of squarks as their production cross section is altered in models with Dirac gluinos.

We note that the structure of these models is quite rich and we did not explore the complete phenomenology of all sectors of the theory. For example, the model has extra scalars as part of the adjoint chiral superfields. Some of these fields could in fact be responsible for obtaining the correct Higgs mass in these models [173,177,227,228]. 


\section{Chapter 7}

\section{Contributions to $b \rightarrow$ sll}

\section{anomalies from $R$-parity violating}

\section{interactions}

\subsection{Introduction}

For a number of years, various experiments have reported anomalies in measurements of semileptonic $B$ decays. For example, consider $R_{K}$ and $R_{K^{*}}$

$$
R_{K^{(*)}}=\frac{\operatorname{Br}\left(B \rightarrow K^{(*)} \mu^{+} \mu^{-}\right)}{\operatorname{Br}\left(B \rightarrow K^{(*)} e^{+} e^{-}\right)} .
$$

As these observables are ratios of branching ratios, they are virtually free of hadronic uncertainties, and thus are excellent tests of lepton flavour universality. The $R_{K}$ ratio, for the dilepton invariant mass squared range 1 to $6 \mathrm{GeV}^{2}$, has been measured 
to be $[229]$

$$
R_{K}=0.745_{-0.074}^{+0.090} \text { (stat) } \pm 0.036 \text { (syst) }, 1<m_{\ell \ell}^{2}<6 \mathrm{GeV}^{2}
$$

by the LHCb collaboration. This represents a $2.6 \sigma$ deviation away from the Standard Model prediction, which is 1 with an uncertainty of $\sim 10^{-2}[230,231]$. Further, the ratio $R_{K^{*}}$ has been measured for two invariant mass squared bins [232]

$$
R_{K^{*}}= \begin{cases}0.66_{-0.07}^{+0.11}(\text { stat }) \pm 0.03(\text { syst }), & 0.045<m_{\ell \ell}^{2}<1.1 \mathrm{GeV}^{2} \\ 0.69_{-0.07}^{+0.11}(\text { stat }) \pm 0.05(\text { syst }), & 1.1<m_{\ell \ell}^{2}<6.0 \mathrm{GeV}^{2}\end{cases}
$$

also by the LHCb collaboration. The Standard Model prediction for these observables varies between 0.878 and 0.944 for the low invariant mass squared bin and 0.990 and 1.010 for the high invariant mass squared bin [232]. The measured values then represent $2.3 \sigma$ and $2.5 \sigma$ deviations for the low and high invariant mass squared bins, respectively. Moreover, some angular distributions also show tension with the Standard Model predictions. In particular, the $P_{5}^{\prime}$ observable [233-235] in the $B \rightarrow K^{*} \mu \mu$ decay as measured by Belle $[236,237]$ and LHCb $[238,239]$ shows a $2.9 \sigma$ discrepancy [240]. Finally, LHCb has also observed a deficit exceeding $3 \sigma$ in another $b \rightarrow s \mu \mu$ transition, namely the $B_{s} \rightarrow \phi \mu^{+} \mu^{-}$decay [241,242].

Taken independently, none of these measurements are in dramatic tension with the Standard Model. However, an interesting feature of these anomalies is that model independent analyses $[240,243-249]$ have shown that new physics contributions to effective four-fermi operators can consistently explain nearly all of them. In fact, a fit of the $b \rightarrow s \ell \ell$ transition data to a set of higher dimensional operators shows that new physics is preferred over the Standard Model at the $5 \sigma$ level [240]. Furthermore, these fits unequivocally demonstrate that one potential way to explain these anomalies is 
to generate new physics contributions to the operator

$$
\left(\bar{s} \gamma_{\alpha} P_{L} b\right)\left(\bar{\mu} \gamma^{\alpha} P_{L} \mu\right)
$$

We also note that there are signs of lepton flavour universality violation in the $b \rightarrow c \ell \nu$ transitions as well. Namely, the ratios of branching ratios $R_{D}$ and $R_{D^{*}}$

$$
R_{D^{(*)}}=\frac{\operatorname{Br}\left(B \rightarrow D^{(*)} \tau \nu\right)}{\operatorname{Br}\left(B \rightarrow D^{(*)} \ell \nu\right)}
$$

where $\ell=e$ or $\mu$, have been measured by Babar [250,251], Belle [252-256], and LHCb [257] and the results seem to be in tension with the Standard Model [258]. However, we do not focus on these discrepancies, although we do briefly discuss them near the end of the chapter.

Many different models featuring new particles, for example leptoquarks (either scalar or vector) that couple to a quark and a lepton, have been proposed to potentially explain these anomalies. Depending on the flavour structure of their couplings, such particles can contribute to the $B$ to $K$ processes, $B$ to $D$ processes, or both [259-274]. In supersymmetric models featuring the $R$-parity violating term $\lambda^{\prime} L Q D^{c}$ in the superpotential, the squarks are in fact leptoquarks. Therefore, such models provide a natural framework to address the anomalies [275-279]. To explain the anomalies in the $b \rightarrow s \mu \mu$ transition we are led to consider loop level contributions as tree level exchange of squarks lead to four-fermi operators with incorrect chirality structures. In these models, there are various kinds of box diagrams that contribute. One class of diagrams involve only intermediate squarks and were considered in a previous work on leptoquarks [262]. In addition, there are diagrams that also involve sleptons which are specific to supersymmetric models. Those contributions 
were considered in [276] which found regions of parameters space that could explain the anomalies and avoid constraints. These regions are characterized by large $\lambda^{\prime}$ couplings and $\mathrm{TeV}$-scale superpartners. As a part of this chapter, we reexamine this parameter space and find new constraints. Finally, in supersymmetric RPV models, there are diagrams involving winos. These have not been considered previously in the literature with regards to the anomalies. Therefore, in this chapter we focus our attention on regions of parameter space where such diagrams give significant contributions. This leads us to a parameter space where the couplings $\lambda_{223}^{\prime}, \lambda_{233}^{\prime}, \lambda_{323}^{\prime}$, and $\lambda_{333}^{\prime}$ are each large. Additionally, the masses of the left-handed squark doublets need to be of order $1 \mathrm{TeV}$, while to avoid various experiment constraints the masses of the right-handed sbottom and the left-handed slepton doublets need to be of order $10 \mathrm{TeV}$.

This chapter is structured as follows. In section 7.2 we compute the contribution of our model to the relevant four-fermi effective operators. We then discuss the region of parameter space which we focus on. In section 7.3 we present various constraints on the model. In particular, the processes $\tau \rightarrow \mu \mu \mu, B_{s}-\bar{B}_{s}$ mixing, $B \rightarrow K^{(*)} \nu \bar{\nu}, Z$ decays to charged leptons, direct LHC searches, and the presence of Landau poles are examined. Finally, we present our results in section 7.4 and we conclude in section 7.5 .

\subsection{Setup and calculations}

The effects of new physics on the decay $b \rightarrow s l l$ can be encoded in contributions to higher dimensional operators. Specifically, the low energy effective Hamiltonian is 
often parametrized as

$$
\mathcal{H}_{\mathrm{eff}}=-\frac{4 G_{F}}{\sqrt{2}} V_{t b} V_{t s}^{*} \frac{\alpha}{4 \pi} \sum_{\ell=e, \mu}\left(C_{9}^{\ell} O_{9}^{\ell}+C_{10}^{\ell} O_{10}^{\ell}+C_{9}^{\ell \ell} O_{9}^{\prime \ell}+C_{10}^{\prime \ell} O_{10}^{\prime \ell}\right)+\text { h.c. }
$$

where $G_{F}$ is Fermi's constant, $V_{i j}$ is the CKM matrix, $\alpha$ is the fine-structure constant, and

$$
\begin{aligned}
O_{9}^{\ell} & =\left(\bar{s} \gamma_{\alpha} P_{L} b\right)\left(\bar{\ell} \gamma^{\alpha} \ell\right), & O_{9}^{\prime \ell} & =\left(\bar{s} \gamma_{\alpha} P_{R} b\right)\left(\bar{\ell} \gamma^{\alpha} \ell\right), \\
O_{10}^{\ell} & =\left(\bar{s} \gamma_{\alpha} P_{L} b\right)\left(\bar{\ell} \gamma^{\alpha} \gamma_{5} \ell\right), & O_{10}^{\prime \ell} & =\left(\bar{s} \gamma_{\alpha} P_{R} b\right)\left(\bar{\ell} \gamma^{\alpha} \gamma_{5} \ell\right) .
\end{aligned}
$$

We find it convenient to switch to the basis described in Ref. [260] where the effective Hamiltonian contains

$$
\mathcal{H}_{\text {eff }} \supset-\frac{4 G_{F}}{\sqrt{2}} V_{t b} V_{t s}^{*} \frac{\alpha}{4 \pi} \sum_{\ell=e, \mu} C_{L L}^{\ell} O_{L L}^{\ell}+\text { h.c. }
$$

where $O_{L L}^{\ell}=\left(O_{9}^{\ell}-O_{10}^{\ell}\right) / 2=\left(\bar{s} \gamma_{\alpha} P_{L} b\right)\left(\bar{\ell} \gamma^{\alpha} P_{L} \ell\right)$ and $C_{L L}^{\ell}=C_{9}^{\ell}-C_{10}^{\ell}$, as well as the analogous operators with the other possible chiral structures. One potential way to explain the anomalies in $b \rightarrow s \mu \mu$ is to generate a large, in absolute value, and negative contribution to $C_{L L}^{\mu} \cdot{ }^{1}$ Using all relevant data, the model independent analysis performed by Ref. [240] finds the best fit value for $C_{L L}^{\mu}$ (assuming that only this coupling receives new physics contributions) to be -1.24 with the $2 \sigma$ range being $-1.76<C_{L L}^{\mu}<-0.74$.

In attempting to explain these anomalies, we consider the $R$-parity violating superpotential term $\lambda_{i j k}^{\prime} L_{i} Q_{j} D_{k}^{c}$. In this expression, the $\lambda^{\prime}$ couplings and the superfields are in a basis where the down-type quark mass matrix is diagonal. To switch

\footnotetext{
${ }^{1}$ Below we often refer to generating large $C_{L L}^{\mu}$. By this we mean large in absolute value and negative.
} 


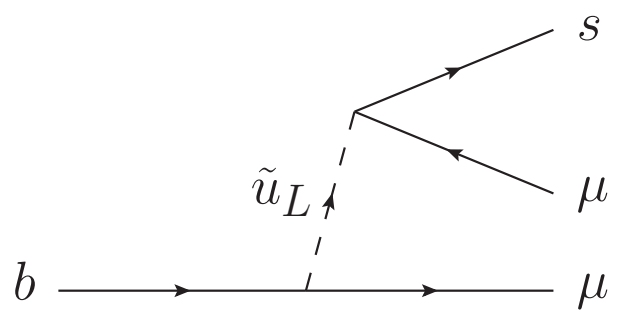

Figure 7.1: Tree level decay for $b \rightarrow s \mu \mu$ involving two $\lambda^{\prime}$ interactions.

to the mass basis, we assume that the scalar soft masses are diagonal in flavour space and apply a rotation to the left-handed up-type superfields. Then, after expanding the superfields in terms of their fermions and sfermions, we get

$$
\begin{aligned}
\mathcal{L} \supset & -\lambda_{i j k}^{\prime}\left(\tilde{\nu}_{i} d_{L j} \bar{d}_{L k}+\tilde{d}_{L j} \nu_{i} \bar{d}_{L k}+\tilde{d}_{R k}^{*} \nu_{i} d_{L j}\right) \\
& +\tilde{\lambda}_{i j k}^{\prime}\left(\tilde{e}_{L i} u_{L j} \bar{d}_{L k}+\tilde{u}_{L j} e_{L i} \bar{d}_{L k}+\tilde{d}_{R k}^{*} e_{L i} u_{L j}\right)+\text { h.c. },
\end{aligned}
$$

where we use 2-spinor notation to denote the fermion fields. In this equation, and throughout the rest of the chapter unless otherwise stated, all repeated indices are assumed to be summed over. We have labeled the couplings involving left-handed down quarks and squarks as $\lambda^{\prime}$ and the couplings involving left-handed up quarks and squarks as $\tilde{\lambda}^{\prime}$. The $\lambda^{\prime}$ and $\tilde{\lambda}^{\prime}$ couplings are related by

$$
\tilde{\lambda}_{i j k}^{\prime}=\lambda_{i l k}^{\prime} V_{j l}^{*}
$$

As shown in figure 7.1, the decay $b \rightarrow s \mu \mu$ can occur at tree level through two $\lambda^{\prime}$ interactions. After integrating out the left-handed up squark we are left with the 
effective Lagrangian

$$
\mathcal{L}_{\text {eff }}=-\frac{\tilde{\lambda}_{2 j 2}^{\prime} \tilde{\lambda}_{2 j 3}^{*}}{2 m_{\tilde{u}_{L j}}^{2}}\left(\bar{s} \gamma^{\alpha} P_{R} b\right)\left(\bar{\mu} \gamma_{\alpha} P_{L} \mu\right)+\text { h.c. }
$$

Notice that this tree level decay necessarily involves a right-handed quark current, and operators involving a right-handed quark current are unable to explain the anomalies. Since we are considering a spectrum which features left-handed up squarks, it is imperative to forbid these diagrams. To do so, we only consider non-zero $\lambda_{i j k}^{\prime}$ for a single value of $k$. This is the same approach as taken in [276]. As will be discussed in section 7.3.1, the couplings with $k=1$ or $k=2$ are excluded in the setup we consider due to $\tau$ decays. However, for the sake of generality, we choose to keep $k$ as a free index in the equations presented in this section. Accordingly, in these equations, the index $k$ is not assumed to be summed over.

With the tree level decay forbidden, the next step is to examine potential loop level processes capable of mediating $b \rightarrow s \mu \mu$. Examples of the different box diagrams that we consider in this chapter are shown in figure $7.2 .^{2}$ First, consider the diagram involving a $W$ boson and a right-handed down squark, figure 7.2a. This diagram is just one of many diagrams involving these two types of particles (if we ignore internal indices then there are four other diagrams, three with a $W$ boson and one with a Goldstone boson). Collectively, we refer to these diagrams as the $W$ loop diagrams. Second, consider the diagram involving a wino and a down quark, figure 7.2b. This diagram is just one of many diagrams involving these two types of particles (if we

\footnotetext{
${ }^{2}$ It is worth noting that there are other potential one loop box diagrams for $b \rightarrow s \mu \mu$ involving $\lambda^{\prime}$ and gauge couplings. However, these diagrams necessarily require the external quarks to be right-handed and thus, after Fierz rearrangements, will generate operators involving a right-handed quark current. Analogous to the tree level diagram, this is undesirable as operators involving a right-handed quark current are unable explain the anomalies. Fortunately, the same trick employed to forbid the tree level diagram, only turning on $\lambda_{i j k}^{\prime}$ for a single value $k$, removes these diagrams as well.
} 


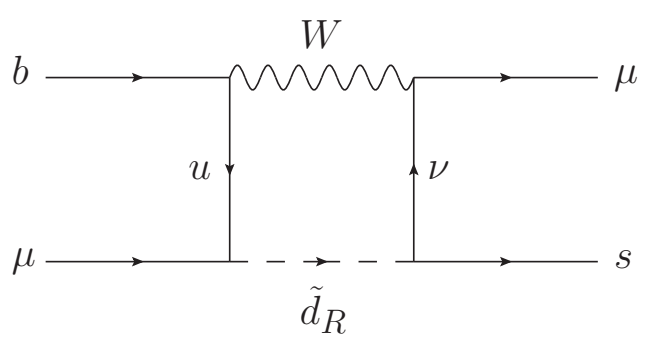

(a)

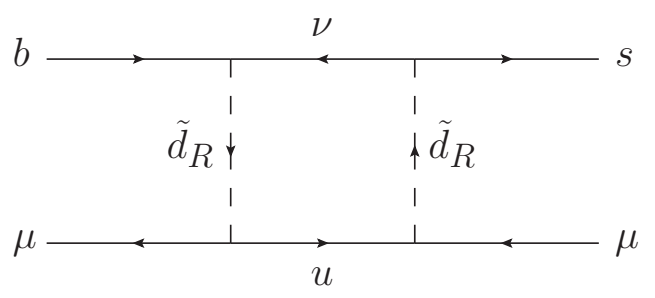

(c)

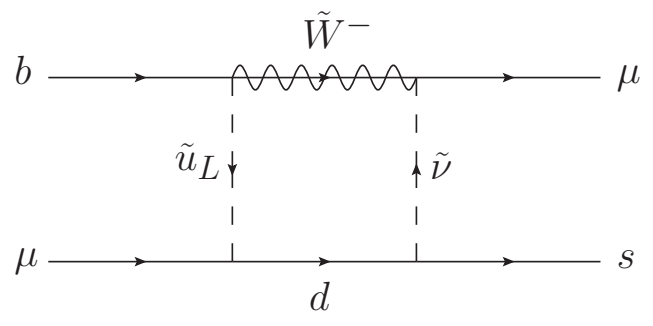

(b)

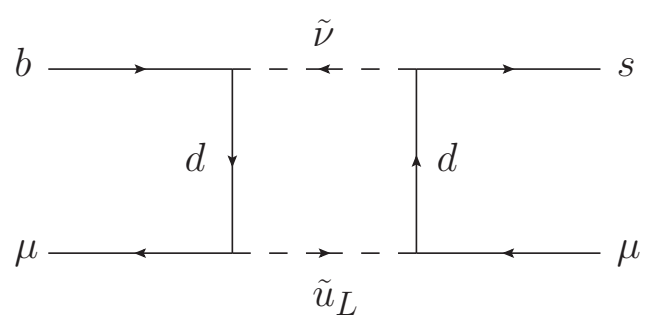

(d)

Figure 7.2: Box diagrams studied in this chapter. Figure 7.2a shows an example $W$ loop diagram, figure $7.2 \mathrm{~b}$ shows an example wino loop diagram, and figures $7.2 \mathrm{c}$ and $7.2 \mathrm{~d}$ show the four- $\lambda^{\prime}$ loop diagrams.

ignore internal indices then there are three other diagrams). Collectively, we refer to these diagrams as the wino loop diagrams. Finally, consider the diagrams involving four $\lambda^{\prime}$ couplings, figures $7.2 \mathrm{c}$ and $7.2 \mathrm{~d}$. Collectively, we refer to these diagrams as the four- $\lambda^{\prime}$ loop diagrams.

Each of these diagrams contribute to $C_{L L}^{\mu}$. Indeed, the $W$ loop diagrams and the four- $\lambda^{\prime}$ loop diagrams have previously been considered in the literature in the context of the $b \rightarrow s \mu \mu$ anomalies. For example, Ref. [262] studied a leptoquark model where equivalent diagrams to the $W$ loop and the four- $\lambda^{\prime}$ loop with two right-handed down squarks were considered. Additionally, Ref. [276] studied an RPV supersymmetry model where the $W$ loop and both four- $\lambda^{\prime}$ loop diagrams were considered. To the best of our knowledge, the wino loop diagrams have not been considered in the context of these anomalies. We now proceed by writing down the contributions of each of these 
diagrams to $C_{L L}^{\mu}$. Although the results for the $W$ loop diagrams and the four- $\lambda^{\prime}$ loop diagrams can be found in the given references, we present them here for completeness.

First, it is convenient to introduce the integrals

$$
\begin{aligned}
D_{0}\left[m_{1}^{2}, m_{2}^{2}, m_{3}^{2}, m_{4}^{2}\right] \equiv & \int \frac{d^{4} k}{(2 \pi)^{4}} \frac{1}{\left(k^{2}-m_{1}^{2}\right)\left(k^{2}-m_{2}^{2}\right)\left(k^{2}-m_{3}^{2}\right)\left(k^{2}-m_{4}^{2}\right)} \\
= & -\frac{i}{16 \pi^{2}}\left(\frac{m_{1}^{2} \log \left(m_{1}^{2}\right)}{\left(m_{1}^{2}-m_{2}^{2}\right)\left(m_{1}^{2}-m_{3}^{2}\right)\left(m_{1}^{2}-m_{4}^{2}\right)}\right. \\
& \left.\quad+\left(m_{1} \leftrightarrow m_{2}\right)+\left(m_{1} \leftrightarrow m_{3}\right)+\left(m_{1} \leftrightarrow m_{4}\right)\right)
\end{aligned}
$$

and

$$
\begin{aligned}
D_{2}\left[m_{1}^{2}, m_{2}^{2}, m_{3}^{2}, m_{4}^{2}\right] \equiv & \int \frac{d^{4} k}{(2 \pi)^{4}} \frac{k^{2}}{\left(k^{2}-m_{1}^{2}\right)\left(k^{2}-m_{2}^{2}\right)\left(k^{2}-m_{3}^{2}\right)\left(k^{2}-m_{4}^{2}\right)} \\
= & -\frac{i}{16 \pi^{2}}\left(\frac{m_{1}^{4} \log \left(m_{1}^{2}\right)}{\left(m_{1}^{2}-m_{2}^{2}\right)\left(m_{1}^{2}-m_{3}^{2}\right)\left(m_{1}^{2}-m_{4}^{2}\right)}\right. \\
& \left.\quad+\left(m_{1} \leftrightarrow m_{2}\right)+\left(m_{1} \leftrightarrow m_{3}\right)+\left(m_{1} \leftrightarrow m_{4}\right)\right)
\end{aligned}
$$

which arise when computing the box diagrams. These are simply the four-point Passarino-Veltman functions where the external momenta have been ignored [149, 150]. These two integrals can also be written so that the arguments of the logarithms are dimensionless ratios of squared masses. We write them in this form to show the symmetry between $m_{1}^{2}, m_{2}^{2}, m_{3}^{2}$, and $m_{4}^{2}$. These integrals also have many well-defined limits when, for example, any of the masses are set to zero or any two masses are set equal. We will often use some of these limits below.

The contribution to $C_{L L}^{\mu}$ due to the $W$ loop diagrams is given by

$$
C_{L L}^{\mu(W)}=\frac{\sqrt{2}}{4 G_{F}} \frac{4 \pi}{\alpha} \frac{1}{V_{t b} V_{t s}^{*}} \frac{1}{i}\left(\frac{g^{2}}{4} \tilde{\lambda}_{2 i k}^{\prime} \lambda_{22 k}^{\prime *} V_{i b} D_{2}\left[m_{\tilde{d}_{R k}}^{2}, m_{u_{i}}^{2}, m_{W}^{2}, 0\right]\right.
$$




$$
\begin{aligned}
& -\frac{g^{2}}{4} \tilde{\lambda}_{2 i k}^{\prime} \tilde{\lambda}_{2 j k}^{\prime *} V_{i b} V_{j s}^{*} D_{2}\left[m_{\tilde{d}_{R k}}^{2}, m_{u_{i}}^{2}, m_{u_{j}}^{2}, m_{W}^{2}\right]+\frac{g^{2}}{4} \lambda_{23 k}^{\prime} \tilde{\lambda}_{2 j k}^{*} V_{j s}^{*} D_{2}\left[m_{\tilde{d}_{R k}}^{2}, m_{u_{j}}^{2}, m_{W}^{2}, 0\right] \\
& \left.-\frac{g^{2}}{4} \lambda_{23 k}^{\prime} \lambda_{22 k}^{*} D_{2}\left[m_{\tilde{d}_{R k}}^{2}, m_{W}^{2}, 0,0\right]+\tilde{\lambda}_{2 i k}^{\prime} \tilde{\lambda}_{2 j k}^{\prime *} V_{i b} V_{j s}^{*} \frac{m_{u_{i}}^{2} m_{u_{j}}^{2}}{2 v^{2}} D_{0}\left[m_{\tilde{d}_{R k}}^{2}, m_{u_{i}}^{2}, m_{u_{j}}^{2}, m_{W}^{2}\right]\right)
\end{aligned}
$$

where $v \approx 174 \mathrm{GeV}$ is the vacuum expectation value of the Standard Model Higgs doublet. In the limit $m_{\tilde{d}_{R k}}^{2} \gg m_{t}^{2}$, this simplifies to

$$
C_{L L}^{\mu(W)}=\frac{\left|\lambda_{23 k}^{\prime}\right|^{2}}{8 \pi \alpha}\left(\frac{m_{t}^{2}}{m_{\tilde{d}_{R k}}^{2}}\right) .
$$

Other combinations of $\lambda^{\prime}$ couplings also contribute to $C_{L L}^{\mu(W)}$ but these are all much smaller. Next, the contribution from the wino loop diagrams is given by the similar expression

$$
\begin{aligned}
& C_{L L}^{\mu(\tilde{W})}=\frac{\sqrt{2}}{4 G_{F}} \frac{4 \pi}{\alpha} \frac{1}{V_{t b} V_{t s}^{*}} \frac{1}{i}\left(\frac{g^{2}}{4} \tilde{\lambda}_{2 i k}^{\prime} \lambda_{22 k}^{\prime *} V_{i b} D_{2}\left[m_{\tilde{W}}^{2}, m_{\tilde{u}_{L i}}^{2}, m_{\tilde{\nu}_{\mu}}^{2}, m_{d_{k}}^{2}\right]\right. \\
& -\frac{g^{2}}{4} \tilde{\lambda}_{2 i k}^{\prime} \tilde{\lambda}_{2 j k}^{\prime *} V_{i b} V_{j s}^{*} D_{2}\left[m_{\tilde{W}}^{2}, m_{\tilde{u}_{L i}}^{2}, m_{\tilde{u}_{L j}}^{2}, m_{d_{k}}^{2}\right]+\frac{g^{2}}{4} \lambda_{23 k}^{\prime} \tilde{\lambda}_{2 j k}^{\prime *} V_{j s}^{*} D_{2}\left[m_{\tilde{W}^{2}}^{2}, m_{\tilde{u}_{L j}}^{2}, m_{\tilde{\nu}_{\mu}}^{2}, m_{d_{k}}^{2}\right] \\
& \left.-\frac{g^{2}}{4} \lambda_{23 k}^{\prime} \lambda_{22 k}^{* *} D_{2}\left[m_{\tilde{W}}^{2}, m_{\tilde{\nu}_{\mu}}^{2}, m_{\tilde{\nu}_{\mu}}^{2}, m_{d_{k}}^{2}\right]\right) .
\end{aligned}
$$

If we make the assumption that the masses of the three left-handed up squarks are degenerate, then this simplifies to

$$
\begin{aligned}
C_{L L}^{\mu(\tilde{W})}= & \frac{\sqrt{2} g^{2} \lambda_{23 k}^{\prime} \lambda_{22 k}^{\prime *}}{64 \pi G_{F} \alpha V_{t b} V_{t s}^{*} m_{\tilde{W}^{2}}^{2}}\left(\frac{1}{x_{\tilde{\nu}_{\mu}}-1}+\frac{1}{x_{\tilde{u}_{L}}-1}\right. \\
& \left.+\frac{\left(x_{\tilde{\nu}_{\mu}}-2 x_{\tilde{\nu}_{\mu}}^{2}+x_{\tilde{u}_{L}}\right) \log \left(x_{\tilde{\nu}_{\mu}}\right)}{\left(x_{\tilde{\nu}_{\mu}}-1\right)^{2}\left(x_{\tilde{\nu}_{\mu}}-x_{\tilde{u}_{L}}\right)}+\frac{\left(x_{\tilde{u}_{L}}-2 x_{\tilde{u}_{L}}^{2}+x_{\tilde{\nu}_{\mu}}\right) \log \left(x_{\tilde{u}_{L}}\right)}{\left(x_{\tilde{u}_{L}}-1\right)^{2}\left(x_{\tilde{u}_{L}}-x_{\tilde{\nu}_{\mu}}\right)}\right)
\end{aligned}
$$

where $x_{\tilde{\nu}_{\mu}}=m_{\tilde{\nu}_{\mu}}^{2} / m_{\tilde{W}}^{2}, x_{\tilde{u}_{L}}=m_{\tilde{u}_{L}}^{2} / m_{\tilde{W}}^{2}$, and we have set $m_{d_{k}}^{2} \rightarrow 0$. Notice that if $x_{\tilde{\nu}_{\mu}}=x_{\tilde{u}_{L}}$, then this expression vanishes due to a super GIM mechanism. Another 


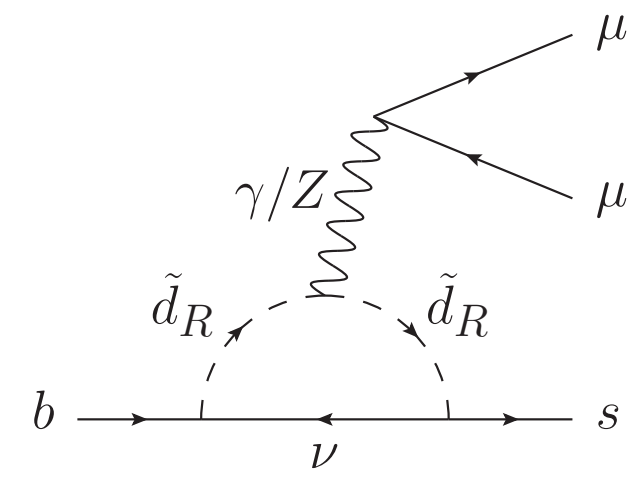

Figure 7.3: An example penguin diagram for $b \rightarrow s \mu \mu$.

relevant limit is $x_{\tilde{\nu}_{\mu}} \gg x_{\tilde{u}_{L}}$, in which case $C_{L L}^{\mu(\tilde{W})}$ further simplifies to

$$
C_{L L}^{\mu(\tilde{W})}=\frac{\sqrt{2} g^{2} \lambda_{23 k}^{\prime} \lambda_{22 k}^{\prime *}}{64 \pi G_{F} \alpha V_{t b} V_{t s}^{*} m_{\tilde{W}}^{2}}\left(\frac{1}{x_{\tilde{u}_{L}}-1}-\frac{\log \left(x_{\tilde{u}_{L}}\right)}{\left(x_{\tilde{u}_{L}}-1\right)^{2}}\right)
$$

which is simply the result of the box diagram with two left-handed up squarks in the loop. Finally, the contribution from the four- $\lambda^{\prime}$ loop diagrams is given by

$$
\begin{aligned}
C_{L L}^{\mu\left(4 \lambda^{\prime}\right)}=\frac{\sqrt{2}}{4 G_{F}} \frac{4 \pi}{\alpha} \frac{1}{V_{t b} V_{t s}^{*}} \frac{1}{4} \lambda_{i 3 k}^{\prime} \lambda_{i 2 k}^{\prime *} \tilde{\lambda}_{2 j k}^{\prime} \tilde{\lambda}_{2 j k}^{\prime *} \frac{1}{i}\left(D_{2}\left[m_{\tilde{d}_{R k}}^{2}, m_{\tilde{d}_{R k}}^{2}, m_{u_{j}}^{2}, 0\right]\right. \\
\left.+D_{2}\left[m_{\tilde{u}_{L j}}^{2}, m_{\tilde{\nu}_{i}}^{2}, m_{d_{k}}^{2}, m_{d_{k}}^{2}\right]\right) .
\end{aligned}
$$

Assuming the masses of the three left-handed up squarks are degenerate and taking the limit $m_{\tilde{d}_{R k}}^{2} \gg m_{t}^{2}$, this simplifies to

$$
C_{L L}^{\mu\left(4 \lambda^{\prime}\right)}=-\frac{\sqrt{2} \lambda_{i 3 k}^{\prime} \lambda_{i 2 k}^{\prime *} \lambda_{2 j k}^{\prime} \lambda_{2 j k}^{\prime *}}{64 \pi G_{F} \alpha V_{t b} V_{t s}^{*}}\left(\frac{1}{m_{\tilde{d}_{R k}}^{2}}+\frac{\log \left(m_{\tilde{\nu}_{i}}^{2} / m_{\tilde{u}_{L}}^{2}\right)}{m_{\tilde{\nu}_{i}}^{2}-m_{\tilde{u}_{L}}^{2}}\right)
$$

So far, we have considered only box diagrams for $b \rightarrow s \mu \mu$. We now consider potential photonic and $Z$ penguin contributions, for which an example diagram is shown in figure 7.3. Starting with the photonic penguin, we determine its contribution 
to $C_{L L}^{\mu}$ as follows. Consider first the generic amplitude for the process $\bar{b} \rightarrow \bar{s} \gamma^{(*)}$

$$
\begin{aligned}
& i \mathcal{M}=i e \epsilon^{\alpha *} \bar{v}_{b}(p) {\left[\gamma^{\beta}\left(g_{\alpha \beta} q^{2}-q_{\alpha} q_{\beta}\right)\left(A_{b 1}^{L} P_{L}+A_{b 1}^{R} P_{R}\right)\right.} \\
&\left.+m_{b} \sigma_{\alpha \beta} i q^{\beta}\left(A_{b 2}^{L} P_{L}+A_{b 2}^{R} P_{R}\right)\right] v_{s}(p-q) .
\end{aligned}
$$

Adapting the results of Ref. [280], who study the process $\mu^{+} \rightarrow e^{+} \gamma^{(*)}$ with $R$-parity violation, we find

$$
\begin{aligned}
& A_{b 1}^{L}=\frac{1}{3} \frac{\lambda_{i 23}^{\prime} \lambda_{i 33}^{\prime *}}{16 \pi^{2}}\left(-\frac{1}{3}\left(\frac{4}{3}+\log \left(\frac{m_{b}^{2}}{m_{\tilde{\nu}_{i}}^{2}}\right)\right) \frac{1}{m_{\tilde{\nu}_{i}}^{2}}+\frac{1}{18 m_{\tilde{b}_{R}}^{2}}\right) \\
& A_{b 1}^{R}=0
\end{aligned}
$$

as well as

$$
\begin{aligned}
A_{b 2}^{L} & =\frac{1}{3} \frac{\lambda_{i 23}^{\prime} \lambda_{i 33}^{\prime *}}{16 \pi^{2}}\left(\frac{1}{12 m_{\tilde{b}_{R}}^{2}}-\frac{1}{6 m_{\tilde{\nu}_{i}}^{2}}\right), \\
A_{b 2}^{R} & =0
\end{aligned}
$$

where we have momentarily considered the case $k=3$. Here, $A_{b 1}^{R}$ and $A_{b 2}^{R}$ are zero because we are only considering non-zero $\lambda_{i j k}^{\prime}$ couplings for a single value of $k$. Next, we match this amplitude onto effective operators. To resolve any potential sign ambiguities, we compare the effective operator for the dipole term with the results present in the literature [281,282]. From these effective operators, we determine a photonic penguin contribution to $C_{L L}^{\mu}$ given by

$$
C_{L L}^{\mu(\gamma)}=-\frac{\sqrt{2} \lambda_{i 33}^{\prime} \lambda_{i 23}^{\prime *}}{12 G_{F} V_{t b} V_{t s}^{*}}\left(-\frac{1}{3}\left(\frac{4}{3}+\log \left(\frac{m_{b}^{2}}{m_{\tilde{\nu}_{i}}^{2}}\right)\right) \frac{1}{m_{\tilde{\nu}_{i}}^{2}}+\frac{1}{18 m_{\tilde{b}_{R}}^{2}}\right)
$$

as well as an equal contribution to $C_{L R}^{\mu}$ as defined in [260]. Notice, however, that $C_{L L}^{e}$ 
and $C_{L R}^{e}$ will receive identical contributions. Thus the photonic penguin diagrams should not have any effect on lepton universality violating observables such as $R_{K^{(*)}}$. On the other hand, they should still affect the other types of variables, such as the various angular observables, used when making the fits for the Wilson coefficients. Regardless, it so happens that, in the setup we consider, all potential contributions from the photonic penguin diagrams are very small. We decide to add $C_{L L}^{\mu(\gamma)}$ to $C_{L L}^{\mu}$ but emphasize that this only has a negligible effect. Finally, we find that the $Z$ penguin diagrams vanish in the limit of zero down-type quark masses.

To explain the anomalies, we need to generate negative contributions to $C_{L L}^{\mu}$. From equation 7.15, we see that the $W$ loop diagrams necessarily give a positive contribution. Next, the term in the large brackets in equation 7.17 is positive for all values of $x_{\tilde{\nu}_{\mu}}$ and $x_{\tilde{u}_{L}}$. Assuming real $\lambda^{\prime}$, which we do for the remainder of this section, we need to take the product $\lambda_{22 k}^{\prime} \lambda_{23 k}^{\prime}>0$ to make $C_{L L}^{\mu(\tilde{W})}$ negative. Further, as previously mentioned, $C_{L L}^{\mu(\tilde{W})}$ vanishes in the limit $x_{\tilde{\nu}_{\mu}}=x_{\tilde{u}_{L}}$ due to a super GIM mechanism. As a result, to increase the magnitude of $C_{L L}^{\mu(\tilde{W})}$ it is beneficial to split the muon sneutrino and left-handed up squark masses. Taking the muon sneutrino mass much larger than the left-handed up squark masses leads to equation 7.18. Finally, by examining equation 7.20 , we see that if $\lambda_{22 k}^{\prime} \lambda_{23 k}^{\prime}>0$, then $C_{L L}^{\mu\left(4 \lambda^{\prime}\right)}$ receives a positive contribution. On the other hand, if we take $\lambda_{12 k}^{\prime} \lambda_{13 k}^{\prime}<0$ or $\lambda_{32 k}^{\prime} \lambda_{33 k}^{\prime}<0$ then this will result in negative contributions to $C_{L L}^{\mu\left(4 \lambda^{\prime}\right)}$.

With these considerations, we envision the following spectrum. The masses of the wino and the three left-handed up squarks are light, of order $1 \mathrm{TeV}$. The product $\lambda_{22 k}^{\prime} \lambda_{23 k}^{\prime}$ is positive and, to enhance the wino loop diagrams, fairly large. As we will see in section 7.3.2, the product $\lambda_{22 k}^{\prime} \lambda_{23 k}^{\prime}$ is highly constrained by $B_{s}-\bar{B}_{s}$ mixing. To get around this constraint, the sfermions which enable $B_{s}-\bar{B}_{s}$ mixing with $\lambda^{\prime}$ 
interactions, the right-handed down squarks and sneutrinos, must be made heavy. We set the masses of these particles to order $10 \mathrm{TeV}$. The $W$ loop diagrams and the four- $\lambda^{\prime}$ loop diagrams proportional to $\lambda_{22 k}^{\prime} \lambda_{23 k}^{\prime}$, which each give positive contributions to $C_{L L}^{\mu}$, are then suppressed. Furthermore, we find that it is still difficult to generate large enough $C_{L L}^{\mu}$ to explain the anomalies in this setup. Thus, we also turn on the product $\lambda_{32 k}^{\prime} \lambda_{33 k}^{\prime}$ and make it negative so that the four- $\lambda^{\prime}$ loop diagrams proportional to this product of couplings then give negative contributions to $C_{L L}^{\mu}$. In fact, if we take $-\lambda_{32 k}^{\prime} \lambda_{33 k}^{\prime}>\lambda_{22 k}^{\prime} \lambda_{23 k}^{\prime}$ then $C_{L L}^{\mu\left(4 \lambda^{\prime}\right)}$ will be negative. However, we must then consider constraints involving taus. One such constraint, examined in section 7.3.1, is $\tau$ decays to a $\mu$ and a meson. There we find that the cases $k=1$ or $k=2$ are ruled out, and we are forced to consider $k=3$. Due to this, the only right-handed down squark which is now relevant is the sbottom. In summary, we consider a light wino, light left-handed up squarks, a heavy right-handed sbottom, heavy sneutrinos, and the four $R$-parity violating couplings $\lambda_{223}^{\prime}, \lambda_{233}^{\prime}, \lambda_{323}^{\prime}, \lambda_{333}^{\prime}$ with $\lambda_{223}^{\prime} \lambda_{233}^{\prime}>0$ and $\lambda_{323}^{\prime} \lambda_{333}^{\prime}<0$.

There are two last points we wish to make before discussing potential constraints. First, we have chosen to turn on $\lambda_{323}^{\prime} \lambda_{333}^{\prime}$ instead of $\lambda_{123}^{\prime} \lambda_{133}^{\prime}$. There are two reasons for making this choice. The first is that if $\lambda_{123}^{\prime} \lambda_{133}^{\prime}$ is taken to be non-zero, then there will be diagrams contributing to $C_{L L}^{e}$. We avoid this since the fits, using all relevant observables, tend to prefer new physics in the muon channel than in the electron channel. Interpreting our results would also become much more challenging. The second reason for this choice of parameters is that by turning on $\lambda_{323}^{\prime} \lambda_{333}^{\prime}$ instead of $\lambda_{123}^{\prime} \lambda_{133}^{\prime}$ we need only to consider weaker constraints involving taus as opposed to stronger constraints involving electrons. For example, in section 7.3.1 we consider constraints from $\tau \rightarrow \mu \mu \mu$. This process is much less constrained than $\mu \rightarrow$ eee. 
Finally, the last point we make is that taking $\lambda_{223}^{\prime} \lambda_{233}^{\prime}>0$ and $\lambda_{323}^{\prime} \lambda_{333}^{\prime}<0$ has an additional benefit, it tends to cause cancellations amongst diagrams contributing to potentially constraining processes. For example, as we will see in section 7.3.2, such cancellations happen in $B_{s}-\bar{B}_{s}$ mixing. We consider these cancellations a feature of the model, as the choice of parameters which lead to them is what is precisely preferred to explain the anomalies.

\subsection{Constraints}

\subsection{1 $\tau$ decays}

The first type of constraints we discuss are those which follow from $\tau$ decays to a $\mu$

and a meson. This type of process was considered in [283] (see also [19]) to bound various combinations of RPV couplings. We will show the results in [283] which are relevant to our parameter space and update the bounds using the latest experimental data.

This type of process can be divided into two subcategories, $\tau \rightarrow \mu V$ and $\tau \rightarrow$ $\mu P$, where $V$ represents a vector meson and $P$ a pseudoscalar. Both types of $\tau$ decays can occur via a tree level exchange of a $\tilde{u}_{L}$ or a $\tilde{d}_{R}$ depending on which meson is in the final state. However, as also noted in [19], we find that stronger constraints come from $\tau$ decays to vector mesons than from $\tau$ decays to pseudoscalars. Particularly, the mesons which give the strongest bounds are $\rho^{0}$ and $\phi$. The branching ratio for the decay $\tau \rightarrow \mu V$ is given by [283]

$$
\operatorname{Br}(\tau \rightarrow \mu V)=\frac{1}{512 \pi}\left|A_{V}\right|^{2} f_{V}^{2} m_{\tau}^{3}\left(1+\frac{m_{V}^{2}}{m_{\tau}^{2}}-2 \frac{m_{V}^{4}}{m_{\tau}^{4}}\right)\left(1-\frac{m_{V}^{2}}{m_{\tau}^{2}}\right) \tau_{\tau}
$$

where $\tau_{\tau}$ is the mean lifetime of the $\tau$ and we have taken the $m_{\mu}^{2} / m_{\tau}^{2} \rightarrow 0$ limit. The 
vector meson decay constant $f_{V}$ is defined by [283]

$$
\left\langle\rho^{0}(p, \epsilon)\left|\bar{u} \gamma_{\alpha} u(0)\right| 0\right\rangle=m_{\rho} f_{\rho} \epsilon_{\alpha}^{*}=-\left\langle\rho^{0}(p, \epsilon)\left|\bar{d} \gamma_{\alpha} d(0)\right| 0\right\rangle
$$

for $\rho^{0}$, and

$$
\left\langle\phi(p, \epsilon)\left|\bar{s} \gamma_{\alpha} s(0)\right| 0\right\rangle=m_{\phi} f_{\phi} \epsilon_{\alpha}^{*}
$$

for $\phi$, with $f_{\rho}=153 \mathrm{MeV}$ and $f_{\phi}=237 \mathrm{MeV}$. Additionally, $A_{V}$ is given by [283]

$$
A_{\rho^{0}}=\frac{\tilde{\lambda}_{3 j 1}^{\prime} \tilde{\lambda}_{2 j 1}^{\prime *}}{m_{\tilde{u}_{L j}}^{2}}-\frac{\tilde{\lambda}_{31 k}^{\prime} \tilde{\lambda}_{21 k}^{\prime *}}{m_{\tilde{d}_{R k}}^{2}}
$$

for $\rho^{0}$, and

$$
A_{\phi}=\frac{\tilde{\lambda}_{3 j 2}^{\prime} \tilde{\lambda}_{2 j 2}^{\prime *}}{m_{\tilde{u}_{L j}}^{2}}
$$

for $\phi$. The current experimental upper limits on the branching ratios for these two processes are $\operatorname{Br}\left(\tau \rightarrow \mu \rho^{0}\right)<1.2 \times 10^{-8}$ and $\operatorname{Br}(\tau \rightarrow \mu \phi)<8.4 \times 10^{-8}$ [284]. These translate into the bounds

$$
\left|\tilde{\lambda}_{3 j 1}^{\prime} \tilde{\lambda}_{2 j 1}^{\prime *}\left(\frac{1 \mathrm{TeV}}{m_{\tilde{u}_{L j}}}\right)^{2}-\tilde{\lambda}_{31 k}^{\prime} \tilde{\lambda}_{21 k}^{\prime *}\left(\frac{1 \mathrm{TeV}}{m_{\tilde{d}_{R k}}}\right)^{2}\right|<0.019
$$

and

$$
\left|\tilde{\lambda}_{3 j 2}^{\prime} \tilde{\lambda}_{2 j 2}^{\prime *}\left(\frac{1 \mathrm{TeV}}{m_{\tilde{u}_{L j}}}\right)^{2}\right|<0.036
$$

respectively. As we are considering the masses of the left-handed up squarks to be 
of order $1 \mathrm{TeV}$, these two bounds are highly constraining. Indeed, explaining the anomalies with the couplings $\lambda_{22 k}^{\prime}, \lambda_{23 k}^{\prime}, \lambda_{32 k}^{\prime}$, and $\lambda_{33 k}^{\prime}$ with $k=1$ or $k=2$ proves to be impossible due to these stringent limits. This is why we are forced to consider the couplings $\lambda_{223}^{\prime}, \lambda_{233}^{\prime}, \lambda_{323}^{\prime}$, and $\lambda_{333}^{\prime}$. Below, we will discuss constraints which would otherwise depend on $m_{\tilde{d}_{R k}}$. However, because of this restriction, we will only mention the right-handed sbottom from here on out.

Other $\tau$ decays which can potentially constrain the parameter space include $\tau \rightarrow \mu \gamma$ and the similar processes $\tau \rightarrow \mu \mu \mu$ and $\tau \rightarrow \mu e^{+} e^{-}$. The processes $\mu \rightarrow e \gamma$ and $\mu \rightarrow e e e$ in the context of RPV supersymmetry are considered in detail in Ref. [280] and we modify their results for $\tau$ decays. First, note that the amplitude for $\tau^{+} \rightarrow \mu^{+} \gamma^{(*)}$ is the same, up to appropriate modifications, as the amplitude given in equation 7.21. The dipole term contributes to the decay $\tau \rightarrow \mu \gamma$ and leads to a branching ratio of $[280]$

$$
\operatorname{Br}(\tau \rightarrow \mu \gamma)=\frac{\alpha m_{\tau}^{5}}{4}\left(\left|A_{\tau 2}^{L}\right|^{2}+\left|A_{\tau 2}^{R}\right|^{2}\right) \tau_{\tau}
$$

where we have again taken the $m_{\mu}^{2} / m_{\tau}^{2} \rightarrow 0$ limit, and [280]

$$
\begin{aligned}
& A_{\tau 2}^{L}=-\frac{\lambda_{2 j 3}^{\prime} \lambda_{3 j 3}^{\prime *}}{64 \pi^{2} m_{\tilde{b}_{R}}^{2}}, \\
& A_{\tau 2}^{R}=0 .
\end{aligned}
$$

Interestingly, $A_{\tau 2}^{L}$ does not depend on the masses of the left-handed up squarks, even though there are diagrams which involve these particles. This is because in the limit $m_{b}^{2} / m_{\tilde{u}_{L}}^{2} \rightarrow 0$ and $m_{\tau}^{2} / m_{\tilde{u}_{L}}^{2} \rightarrow 0$ there is an exact cancellation amongst the individual diagrams. Also worth noting is that to reach $A_{\tau 2}^{L}$ shown above we have taken the 


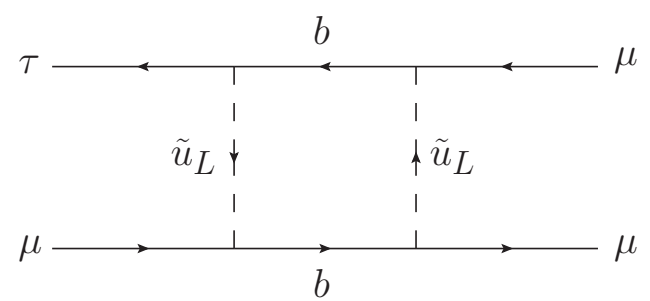

(a)

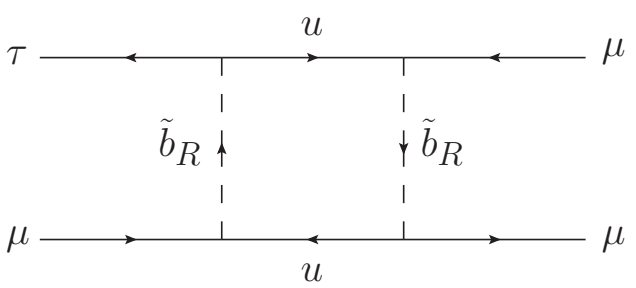

(b)

Figure 7.4: One loop box diagrams contributing to $\tau \rightarrow \mu \mu \mu$.

$m_{t}^{2} / m_{\tilde{b}_{R}}^{2} \rightarrow 0$ limit. The branching ratio then becomes

$$
\operatorname{Br}(\tau \rightarrow \mu \gamma)=\frac{\alpha m_{\tau}^{5}}{16384 \pi^{4} m_{\tilde{b}_{R}}^{4}}\left|\lambda_{223}^{\prime} \lambda_{323}^{\prime *}+\lambda_{233}^{\prime} \lambda_{333}^{\prime *}\right|^{2} \tau_{\tau}
$$

and this, using the current experimental upper limit $\operatorname{Br}(\tau \rightarrow \mu \gamma)<4.4 \times 10^{-8}$ [284], leads to the bound

$$
\left|\lambda_{223}^{\prime} \lambda_{323}^{\prime *}+\lambda_{233}^{\prime} \lambda_{333}^{\prime *}\right|<1.1\left(\frac{m_{\tilde{b}_{R}}}{1 \mathrm{TeV}}\right)^{2}
$$

Since we are considering $m_{\tilde{b}_{R}}$ to be of order $10 \mathrm{TeV}$, we find no constraints from $\tau \rightarrow \mu \gamma$

Next we consider the decay $\tau^{+} \rightarrow \mu^{+} \mu^{+} \mu^{-}$. This decay receives three different types of contributions, photonic and $Z$ penguin diagrams and box diagrams with four $\lambda^{\prime}$ couplings. We write this as

$$
i \mathcal{M}=i \mathcal{M}^{\gamma}+i \mathcal{M}^{Z}+i \mathcal{M}^{4 \lambda^{\prime}}
$$

The photonic penguin amplitude $i \mathcal{M}^{\gamma}$ is given by

$$
i \mathcal{M}^{\gamma}=i e^{2}\left[\bar{v}_{\tau}(p)\left(\gamma_{\alpha}\left(A_{\tau 1}^{L} P_{L}+A_{\tau 1}^{R} P_{R}\right)+m_{\tau} \sigma_{\alpha \beta} \frac{i q^{\beta}}{q^{2}}\left(A_{\tau 2}^{L} P_{L}+A_{\tau 2}^{R} P_{R}\right)\right) v_{\mu}\left(p_{2}\right)\right]
$$




$$
\left[\bar{u}_{\mu}\left(p_{3}\right) \gamma^{\alpha} v_{\mu}\left(p_{1}\right)\right]-\left(p_{1} \leftrightarrow p_{2}\right)
$$

The functions $A_{\tau 2}^{L}$ and $A_{\tau 2}^{R}$ are still given by equations 7.35 and 7.36 , respectively, and $A_{\tau 1}^{R}=0$. The function $A_{\tau 1}^{L}$ is similar in nature to equation 7.22 but is slightly more complicated. Its exact form can be determined from the results in [280]. We do note though that, unlike $A_{\tau 2}^{L}, A_{\tau 1}^{L}$ does depend on the masses of the left-handed up squarks. Next, the amplitude for the $Z$ penguin is given by

$i \mathcal{M}^{Z}=i \frac{g^{2}}{32 \pi^{2} c_{W}^{2} m_{Z}^{2}} B_{32}^{2}\left[\bar{v}_{\tau}(p) \gamma^{\alpha} P_{L} v_{\mu}\left(p_{2}\right)\right]\left[\bar{u}_{\mu}\left(p_{3}\right) \gamma_{\alpha}\left(\kappa_{L} P_{L}+\kappa_{R} P_{R}\right) v_{\mu}\left(p_{1}\right)\right]-\left(p_{1} \leftrightarrow p_{2}\right)$

where $\kappa_{L}=-\frac{1}{2}+s_{W}^{2}, \kappa_{R}=s_{W}^{2}, c_{W}=\cos \theta_{W}, s_{W}=\sin \theta_{W}$, and the function $B_{32}^{2}$ is given in equation 7.57 with $m_{Z}^{2} \rightarrow 0$. Finally, consider the two box diagrams shown in figure 7.4. The amplitude for these two diagrams is

$$
i \mathcal{M}^{4 \lambda^{\prime}}=i C_{\tau}\left[\bar{v}_{\tau}(p) \gamma^{\alpha} P_{L} v_{\mu}\left(p_{2}\right)\right]\left[\bar{u}_{\mu}\left(p_{3}\right) \gamma_{\alpha} P_{L} v_{\mu}\left(p_{1}\right)\right]-\left(p_{1} \leftrightarrow p_{2}\right)
$$

where $C_{\tau}$ is given by

$$
C_{\tau}=-\frac{1}{4} \tilde{\lambda}_{2 i 3}^{\prime} \tilde{\lambda}_{2 i 3}^{\prime *} \tilde{\lambda}_{2 j 3}^{\prime} \tilde{\lambda}_{3 j 3}^{\prime *} \frac{1}{i}\left(D_{2}\left[m_{\tilde{u}_{L i}}^{2}, m_{\tilde{u}_{L j}}^{2}, m_{b}^{2}, m_{b}^{2}\right]+D_{2}\left[m_{\tilde{b}_{R}}^{2}, m_{\tilde{b}_{R}}^{2}, m_{u_{i}}^{2}, m_{u_{j}}^{2}\right]\right)
$$

Assuming mass degenerate left-handed up squarks, $m_{\tilde{u}_{L}}^{2} \gg m_{b}^{2}$, and $m_{\tilde{b}_{R}}^{2} \gg m_{t}^{2}$, this simplifies to

$$
C_{\tau}=\frac{\lambda_{2 i 3}^{\prime} \lambda_{2 i 3}^{*} \lambda_{2 j 3}^{\prime} \lambda_{3 j 3}^{\prime *}}{64 \pi^{2}}\left(\frac{1}{m_{\tilde{u}_{L}}^{2}}+\frac{1}{m_{\tilde{b}_{R}}^{2}}\right)
$$

To compute potential limits from $\tau \rightarrow \mu \mu \mu$, we first write the amplitude in Math- 
ematica with the assistance of FeynCalc [285,286]. Then, also using FeynCalc, we square the amplitude and sum and average over spins. Finally, we numerically integrate over the three-body phase space to determine the partial width. This value is then multiplied by the mean lifetime of the $\tau$ to determine the branching ratio, which is then compared to the experimental upper limit $\operatorname{Br}(\tau \rightarrow \mu \mu \mu)<2.1 \times 10^{-8}$ [284].

Potential constraints from $\tau^{+} \rightarrow \mu^{+} e^{+} e^{-}$are determined in a completely analogous fashion. Although, for this decay, only the photonic and $Z$ penguin diagrams contribute, whose amplitudes are similar to equations 7.40 and 7.41 , respectively, with appropriate modifications. The branching ratio is again computed with the assistance of FeynCalc and the result is compared with the experimental upper limit $\operatorname{Br}\left(\tau \rightarrow \mu e^{+} e^{-}\right)<1.8 \times 10^{-8}[284]$.

The last type of process we consider involving taus is the decay $\tau \rightarrow K \nu$. This decay, which occurs in the Standard Model through a $W$ boson, can also potentially occur via a tree level exchange of a right-handed sbottom with two $\lambda^{\prime}$ interactions. However, because we consider the right-handed sbottom to be heavy, we find no meaningful constraints from this decay.

\subsection{2 $B$ mesons}

Strong constraints on the parameters in our model can be derived from $B_{s}-\bar{B}_{s}$ mixing. Particularly, the $\lambda^{\prime}$ interactions induce $B_{s}-\bar{B}_{s}$ mixing via one loop box diagrams with either two right-handed sbottoms or two sneutrinos in the loop. Additionally, $B_{s}-\bar{B}_{s}$ mixing can also be induced by a one loop box diagram with two left-handed up squarks and two winos in the loop. It is useful to define the effective Lagrangian for this process

$$
\mathcal{L}_{\text {eff }}=C_{B_{s}}^{\mathrm{SM}(\mathrm{NP})}\left(\bar{s} \gamma^{\alpha} P_{L} b\right)\left(\bar{s} \gamma_{\alpha} P_{L} b\right)+\text { h.c. }
$$


where $C_{B_{s}}^{\mathrm{SM}(\mathrm{NP})}$ is generated by the Standard Model (new physics). Explicitly, these are given by

$$
C_{B_{s}}^{\mathrm{SM}}=-\frac{g^{4}}{128 \pi^{2} m_{W}^{2}}\left(V_{t b} V_{t s}^{*}\right)^{2} S_{0}\left(x_{t}\right)
$$

with $x_{t}=m_{t}^{2} / m_{W}^{2}, m_{t}=m_{t}\left(m_{t}\right) \approx 162.3 \mathrm{GeV}$, and $S_{0}\left(x_{t}\right)=\frac{x_{t}\left(4-11 x_{t}+x_{t}^{2}\right)}{4\left(1-x_{t}\right)^{2}}-\frac{3 x_{t}^{3} \log \left(x_{t}\right)}{2\left(1-x_{t}\right)^{3}} \approx$ 2.30 , and

$$
\begin{aligned}
C_{B_{s}}^{\mathrm{NP}} & =\frac{1}{8} \lambda_{i 33}^{\prime} \lambda_{i 23}^{* *} \lambda_{j 33}^{\prime} \lambda_{j 23}^{\prime *} \frac{1}{i}\left(D_{2}\left[m_{\tilde{b}_{R}}^{2}, m_{\tilde{b}_{R}}^{2}, 0,0\right]+D_{2}\left[m_{\tilde{\nu}_{i}}^{2}, m_{\tilde{\nu}_{j}}^{2}, m_{b}^{2}, m_{b}^{2}\right]\right) \\
& +\frac{g^{4}}{8} V_{i b} V_{i s}^{*} V_{j b} V_{j s}^{*} \frac{1}{i} D_{2}\left[m_{\tilde{u}_{L i}}^{2}, m_{\tilde{u}_{L j}}^{2}, m_{\tilde{W}^{2}}^{2}, m_{\tilde{W}^{2}}^{2}\right] .
\end{aligned}
$$

In the limit of degenerate left-handed up squarks (which removes the wino contribution due to a super GIM mechanism) and $m_{\tilde{\nu}}^{2} \gg m_{b}^{2}, C_{B_{s}}^{\mathrm{NP}}$ simplifies to

$$
C_{B_{s}}^{\mathrm{NP}}=-\frac{\lambda_{i 33}^{\prime} \lambda_{i 23}^{\prime *} \lambda_{j 33}^{\prime} \lambda_{j 23}^{\prime *}}{128 \pi^{2}}\left(\frac{1}{m_{\tilde{b}_{R}}^{2}}+\frac{\log \left(m_{\tilde{\nu}_{i}}^{2} / m_{\tilde{\nu}_{j}}^{2}\right)}{m_{\tilde{\nu}_{i}}^{2}-m_{\tilde{\nu}_{j}}^{2}}\right)
$$

Notice the $\lambda^{\prime}$ dependence of this equation. The choice of parameters $\lambda_{223}^{\prime} \lambda_{233}^{\prime}>0$ and $\lambda_{323}^{\prime} \lambda_{333}^{\prime}<0$, initially motivated to achieve large values for $C_{L L}^{\mu}$, causes cancellations amongst the various diagrams. This is an example of the cancellations mentioned at the very end of section 7.2. Importantly, these cancellations help lessen the constraints coming from $B_{s}-\bar{B}_{s}$ mixing. Again, we consider this a feature of the model, as the choice of parameters which lead to these cancellations is what is precisely preferred by $C_{L L}^{\mu}$. To constrain the relevant parameters, we follow the UT $f$ it collaboration [287] and define

$$
C_{B_{s}} e^{2 i \phi_{B_{s}}}=\frac{\left\langle B_{s}^{0}\left|H_{\mathrm{eff}}^{\mathrm{full}}\right| \bar{B}_{s}^{0}\right\rangle}{\left\langle B_{s}^{0}\left|H_{\mathrm{eff}}^{\mathrm{SM}}\right| \bar{B}_{s}^{0}\right\rangle}
$$


We then have that $C_{B_{s}}$ and $\phi_{B_{s}}$ are given by

$$
C_{B_{s}}=\left|1+\frac{C_{B_{s}}^{\mathrm{NP}}}{C_{B_{s}}^{\mathrm{SM}}}\right| \quad \text { and } \quad \phi_{B_{s}}=\frac{1}{2} \operatorname{Arg}\left(1+\frac{C_{B_{s}}^{\mathrm{NP}}}{C_{B_{s}}^{\mathrm{SM}}}\right) .
$$

The $2 \sigma$ bounds on these two values, which can be found on the UT $f$ it collaboration's website, are given by $0.899<C_{B_{s}}<1.252$ and $-1.849^{\circ}<\phi_{B_{s}}<1.959^{\circ}$. We find that, even with the cancellations between the diagrams, the constraint on $C_{B_{s}}$ still requires us to take $m_{\tilde{b}_{R}}$ and $m_{\tilde{\nu}}$ of order $10 \mathrm{TeV}$ if we want the product $\lambda_{223}^{\prime} \lambda_{233}^{\prime}$ to be large.

The next decay we consider is $B \rightarrow K^{(*)} \nu \bar{\nu}$ which results from $b \rightarrow s \nu \bar{\nu}$. The quark level decay can potentially occur by a tree level exchange of a right-handed sbottom with two $\lambda^{\prime}$ interactions. It is useful to define the effective Lagrangian for this process

$$
\mathcal{L}_{\text {eff }}=C_{b \rightarrow s \nu_{i} \bar{\nu}_{j}}^{\mathrm{SM}(\mathrm{NP})}\left(\bar{s} \gamma^{\alpha} P_{L} b\right)\left(\bar{\nu}_{i} \gamma_{\alpha} P_{L} \nu_{j}\right)+\text { h.c. }
$$

where $C_{b \rightarrow s \nu_{i} \bar{\nu}_{j}}^{\mathrm{SM}(\mathrm{NP})}$ is generated by the Standard Model (new physics). Explicitly, these are given by

$$
C_{b \rightarrow s \nu_{i} \bar{\nu}_{j}}^{\mathrm{SM}}=-\delta_{i j} \frac{g^{4}}{16 \pi^{2} m_{W}^{2}} V_{t b} V_{t s}^{*} X_{0}\left(x_{t}\right)
$$

with $x_{t}$ defined as before and $X_{0}\left(x_{t}\right)=\frac{x_{t}\left(x_{t}+2\right)}{8\left(x_{t}-1\right)}+\frac{3 x_{t}\left(x_{t}-2\right)}{8\left(x_{t}-1\right)^{2}} \log \left(x_{t}\right) \approx 1.48$, and

$$
C_{b \rightarrow s \nu_{i} \bar{\nu}_{j}}^{\mathrm{NP}}=\frac{\lambda_{j 33}^{\prime} \lambda_{i 23}^{\prime *}}{2 m_{\tilde{b}_{R}}^{2}}
$$

Next, consider the ratio $R_{B \rightarrow K^{(*)} \nu \bar{\nu}}=\Gamma^{\mathrm{SM}+\mathrm{NP}}\left(B \rightarrow K^{(*)} \nu \bar{\nu}\right) / \Gamma^{\mathrm{SM}}\left(B \rightarrow K^{(*)} \nu \bar{\nu}\right)$. In 
terms of $C_{b \rightarrow s \nu_{i} \bar{\nu}_{j}}^{\mathrm{SM}}$ and $C_{b \rightarrow s \nu_{i} \bar{\nu}_{j}}^{\mathrm{NP}}$, it is given by

$$
R_{B \rightarrow K^{(*)} \nu \bar{\nu}}=\frac{\sum_{i=1}^{3}\left|C_{b \rightarrow s \nu_{i} \bar{\nu}_{i}}^{\mathrm{SM}}+C_{b \rightarrow s \nu_{i} \bar{\nu}_{i}}^{\mathrm{NP}}\right|^{2}+\sum_{i, j=1}^{3}\left(1-\delta_{i j}\right)\left|C_{b \rightarrow s \nu_{i} \bar{\nu}_{j}}^{\mathrm{NP}}\right|^{2}}{\sum_{i=1}^{3}\left|C_{b \rightarrow s \nu_{i} \bar{\nu}_{i}}\right|^{2}} .
$$

The Belle search [288] provides 90\% CL upper bounds $R_{B \rightarrow K \nu \bar{\nu}}<3.9$ and $R_{B \rightarrow K^{*} \nu \bar{\nu}}<$ 2.7 on these ratios. We determine constraints on our parameter space from the limit on $R_{B \rightarrow K^{*} \nu \bar{\nu}}$.

Another potentially constraining process is the decay $B \rightarrow X_{\bar{s}} \gamma$ corresponding to the decay $\bar{b} \rightarrow \bar{s} \gamma$. The amplitude for the quark level process is given in equation 7.21 where, due to the photon being on-shell, only the dipole term contributes. We see that this amplitude depends on $A_{b 2}^{L}$, given in equation 7.24, which is itself proportional to the inverse squared masses of the right-handed sbottom and sneutrinos. Because we take these particles to be heavy, we find no constraints from these decays.

Finally, we also examined the decays $B \rightarrow \tau \nu$ and $B \rightarrow \mu \nu$. Both these decays occur in the Standard Model through a $W$ boson, although the latter decay is highly suppressed due to angular momentum conservation. They can also potentially occur as a result of a tree level right-handed sbottom exchange with two $\lambda^{\prime}$ interactions. However, because we take the mass of the right-handed sbottom to be heavy, we find no constraints from these two decays.

\subsection{3 $Z$ decays}

Loop level processes involving the right-handed sbottom and left-handed up squarks can potentially shift the partial width of the $Z$ to same flavour charged leptons or induce $Z$ decays to opposite flavour charged leptons. Example one loop Feynman 


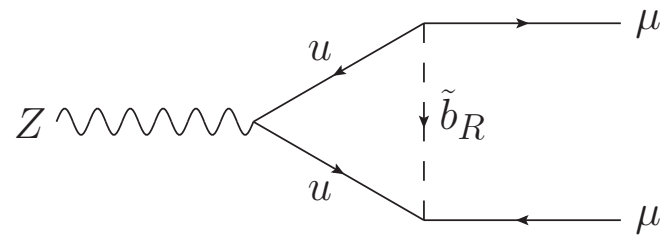

(a)

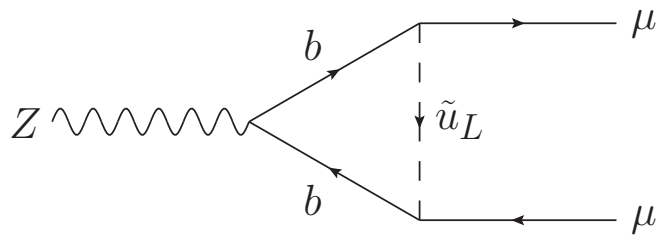

(b)

Figure 7.5: Example one loop Feynman diagrams contributing to $Z \rightarrow \mu \mu$.

diagrams are shown in figure 7.5. These diagrams contribute to the amplitude

$$
i \mathcal{M}=i \frac{g}{32 \pi^{2} c_{W}} B_{i j} \epsilon^{\alpha} \bar{u}_{e_{i}} \gamma_{\alpha} P_{L} v_{e_{j}}
$$

where $B_{i j}=B_{i j}^{1}+B_{i j}^{2}+B_{i j}^{3}$ and

$$
\begin{aligned}
B_{i j}^{1} & =\sum_{l=1}^{2} \tilde{\lambda}_{j l 3}^{\prime} \tilde{\lambda}_{i l 3}^{\prime *} \frac{m_{Z}^{2}}{m_{\tilde{b}_{R}}^{2}}\left[\left(1-\frac{4}{3} s_{W}^{2}\right)\left(\log \left(\frac{m_{Z}^{2}}{m_{\tilde{b}_{R}}^{2}}\right)-i \pi-\frac{1}{3}\right)+\frac{s_{W}^{2}}{9}\right] \\
B_{i j}^{2} & =3 \tilde{\lambda}_{j 33}^{\prime} \tilde{\lambda}_{i 33}^{\prime *}\left\{\frac{m_{t}^{2}}{m_{\tilde{b}_{R}}^{2}}\left(-\log \left(\frac{m_{t}^{2}}{m_{\tilde{b}_{R}}^{2}}\right)-1\right)\right. \\
& \left.+\frac{m_{Z}^{2}}{18 m_{\tilde{b}_{R}}^{2}}\left[\left(11-10 s_{W}^{2}\right)+\left(6-8 s_{W}^{2}\right) \log \left(\frac{m_{t}^{2}}{m_{\tilde{b}_{R}}^{2}}\right)+\frac{1}{10}\left(-9+16 s_{W}^{2}\right) \frac{m_{Z}^{2}}{m_{t}^{2}}\right]\right\}, \\
B_{i j}^{3} & =\sum_{l=1}^{3} \tilde{\lambda}_{j l 3}^{\prime} \tilde{\lambda}_{i l 3}^{\prime *} \frac{m_{Z}^{2}}{m_{\tilde{u}_{L l}}^{2}}\left[\left(-\frac{2}{3} s_{W}^{2}\right)\left(\log \left(\frac{m_{Z}^{2}}{m_{\tilde{u}_{L l}}^{2}}\right)-i \pi-\frac{1}{2}\right)+\left(-\frac{1}{6}+\frac{1}{9} s_{W}^{2}\right)\right] .
\end{aligned}
$$

The function $B_{i j}^{1}$ is the contribution from the diagrams with a right-handed bottom squark and an up or charm quark in the loop. The function $B_{i j}^{2}$ is the contribution from the diagrams with a right-handed bottom squark and a top quark in the loop. These two functions match the results presented in [262], although we have retained additional terms in $B_{i j}^{2}$. The final function $B_{i j}^{3}$ is the contribution from the diagrams with a left-handed up squark and a bottom quark in the loop. 
For the decays $Z \rightarrow \mu \mu$ and $Z \rightarrow \tau \tau$, we derive bounds by demanding that the interference term in the partial width computation between the Standard Model tree level diagram and the one loop contribution presented above is less than twice the experimental uncertainty on the partial width as given in [284]. This leads to the bounds

$$
\left|\operatorname{Re}\left[B_{22}\right]\right|<0.32 \text { and }\left|\operatorname{Re}\left[B_{33}\right]\right|<0.39
$$

The decays $Z \rightarrow \mu \tau$ are bounded by demanding that the one loop contribution does not lead to a branching ratio larger than the experimental upper limit $\operatorname{Br}(Z \rightarrow \mu \tau)<$ $1.2 \times 10^{-5}[284]$. This results in the bound

$$
\sqrt{\left|B_{23}\right|^{2}+\left|B_{32}\right|^{2}}<2.1
$$

\subsubsection{Other possible decays}

The right-handed sbottom and $\lambda^{\prime}$ couplings can also induce several different tree level decays of $D$ mesons. For example, potential constraints can be derived from

examining the decay $D^{0} \rightarrow \mu \mu$, the ratio of branching ratios $R_{D^{+}}^{(*)}=\operatorname{Br}\left(D^{+} \rightarrow\right.$ $\left.\mu^{+} \nu \bar{K}^{0(*)}\right) / \operatorname{Br}\left(D^{+} \rightarrow e^{+} \nu \bar{K}^{0(*)}\right)$ and $R_{D^{0}}=\operatorname{Br}\left(D^{0} \rightarrow \mu^{+} \nu \bar{K}^{-}\right) / \operatorname{Br}\left(D^{0} \rightarrow e^{+} \nu \bar{K}^{-}\right)$, and the decays $D_{s} \rightarrow \tau \nu$ and $D_{s} \rightarrow \mu \nu$. However, because we take the mass of the right-handed sbottom to be large, we find that none of these processes constrain our parameter space.

The last type of processes we consider are upsilon decays to charged lepton pairs, $\Upsilon(1 S) \rightarrow e_{i}^{-} e_{j}^{+}$. The corresponding quark level process $b \bar{b} \rightarrow e_{i}^{-} e_{j}^{+}$can potentially be induced by a tree level exchange of left-handed up squarks and two $\lambda^{\prime}$ interactions. Integrating out the left-handed up squarks, we are left with the following effective 
Lagrangian

$$
\mathcal{L}_{\mathrm{eff}}=-\frac{\tilde{\lambda}_{j l 3}^{\prime} \tilde{\lambda}_{i l 3}^{\prime *}}{2 m_{\tilde{u}_{L l}}^{2}}\left(\bar{b} \gamma^{\alpha} P_{R} b\right)\left(\bar{e}_{i} \gamma_{\alpha} P_{L} e_{j}\right)
$$

Using this effective Lagrangian we can compute the branching ratio for the decay $\Upsilon \rightarrow \mu \tau$ as well potential modifications to the ratio of branching ratios $\operatorname{Br}(\Upsilon \rightarrow$ $\mu \mu) / \operatorname{Br}(\Upsilon \rightarrow e e)$ and $\operatorname{Br}(\Upsilon \rightarrow \tau \tau) / \operatorname{Br}(\Upsilon \rightarrow e e)$. However, we find that the experimental upper limit on $\operatorname{Br}(\Upsilon \rightarrow \mu \tau)$ is not stringent enough and that the decays $\Upsilon \rightarrow e_{i}^{-} e_{i}^{+}$are not measured precisely enough to give any constraints on our parameter space.

\subsubsection{Collider searches}

The next type of constraint we discuss is direct LHC searches for pair produced up squarks subsequently decaying by $\lambda^{\prime}$ interactions. Provided the up squarks are light enough, this process at the LHC would look like $p p \rightarrow \tilde{u}_{L} \tilde{u}_{L}^{*} \rightarrow \ell^{+} \ell^{-} j j$ where, in our case, the two individual leptons can be either muons or taus, and both jets are b-jets. Thus, the possible signatures are two opposite sign muons, an opposite sign muon and tau pair, or two opposite sign taus, together with two b-jets.

There have been several ATLAS and CMS searches looking for these types of topologies, of which one of the most recent is [289]. This is an ATLAS search with centre of mass energy $\sqrt{s}=13 \mathrm{TeV}$ and integrated luminosity $36.1 \mathrm{fb}^{-1}$. It considers stop pair production with the stops decaying by $\lambda^{\prime}$ interactions. The final state topologies it considers are $\ell^{+} \ell^{-} j j$ where $\ell=e$ or $\mu$ and both jets are b-jets. The search presents lower limits for stop masses in the $\operatorname{Br}(\tilde{t} \rightarrow b e)+\operatorname{Br}(\tilde{t} \rightarrow b \mu)+\operatorname{Br}(\tilde{t} \rightarrow b \tau)=1$ plane. To extract limits from this search, we first make the simplifying assumption that the efficiencies to pass the cuts (which require one of $e e, e \mu$, or $\mu \mu$ ) are zero if 
either stop decays to a $\tau$ and a $b$. Then, using the exclusion plot, the provided $95 \%$ CL upper limit on the number of beyond the Standard Model (BSM) signals, and the stop pair production cross section which we compute using NNLL-fast [199-202], we can determine the efficiencies for both stops decaying to a $\mu$ and $b$. Once we have the efficiencies, determining limits on our model is straightforward.

To do this, we first determine the production cross sections for the three individual up squarks. For simplicity, we use the pair production cross section for stops for the first two generations as well. This is equivalent to assuming a heavy gluino. Then, we compute the branching ratios for our up squarks to decay to a $\mu$ and a $b$. Here, we consider the decays $\tilde{u}_{L} \rightarrow \mu b, \tilde{u}_{L} \rightarrow \tau b$, and $\tilde{u}_{L} \rightarrow \tilde{W} q$ where the last decay includes both neutral and charged winos. For large values of the $\lambda^{\prime}$ couplings $\left(\lambda^{\prime} \gtrsim 1\right)$, the first two decays dominate. We then compute the number of expected signals by multiplying the integrated luminosity, the cross sections, the efficiencies, and the squared branching ratios for $\tilde{u}_{L} \rightarrow \mu b$. Comparing this number to the provided 95\% CL upper limit on the number of BSM signals, we determine whether points in parameter space are excluded.

It is worth mentioning that we also examined experimental searches looking for the final state $\tau^{+} \tau^{-} j j$ where again the jets are b-jets. One of the most recent searches looking for this final state is the CMS search [290]. However, this search fails to provide any additional constraints in the parameter space we examine. This is simply because these types of searches provide weaker limits than searches looking for $\ell^{+} \ell^{-} j j(\ell=e$ or $\mu)$ due to the difficulty in reconstructing taus. 


\subsubsection{Landau poles}

To generate large values of $C_{L L}^{\mu}$, we will need to take the four $\lambda^{\prime}$ couplings under consideration to be fairly large. This will then result in Landau poles below the Planck scale. To calculate the energy scales of these Landau poles, we use the following procedure. First, we evolve the three gauge couplings and the top, bottom, and tau Yukawa couplings up to the left-handed up squarks mass scale using the Standard Model beta functions. From there, we evolve these parameters and the four $\lambda^{\prime}$ couplings up to the right-handed sbottom and sneutrino mass scale. The beta functions used for this evolution are the one loop RPVMSSM beta functions [291], except with the following modification. As some of the sparticle masses are at the very top of this evolution scale, we remove their effects on the beta functions. Precisely, we remove the effects on the beta functions due to the sfermions coming from the superfields $U^{c}, D^{c}, L$, and $E^{c}$. Lastly, we evolve the parameters upwards from this scale using the full one loop RPVMSSM beta functions and determine the Landau pole accordingly. The beta functions used to evolve the parameters from the left-handed up squarks mass scale to the right-handed sbottom and sneutrino mass scale and from the right-handed sbottom and sneutrino mass scale upwards are given in appendix A.

\subsection{Results}

Our results are presented in the four plots in figure 7.6. In these plots, we show solid contours of constant values of $C_{L L}^{\mu}$. Also shown are dashed contours representing energy scales in $\mathrm{TeV}$ at which Landau poles occur. In addition, we find that relevant parameter space is excluded by the processes $\tau \rightarrow \mu \mu \mu, B_{s}-\bar{B}_{s}$ mixing, and $B \rightarrow$ $K^{(*)} \nu \bar{\nu}$, as well as direct LHC searches. In making these plots, we have taken $\lambda_{223}^{\prime}$, $\lambda_{233}^{\prime}$, and $\lambda_{323}^{\prime}$ positive and $\lambda_{333}^{\prime}$ negative. There are essentially identical plots with 


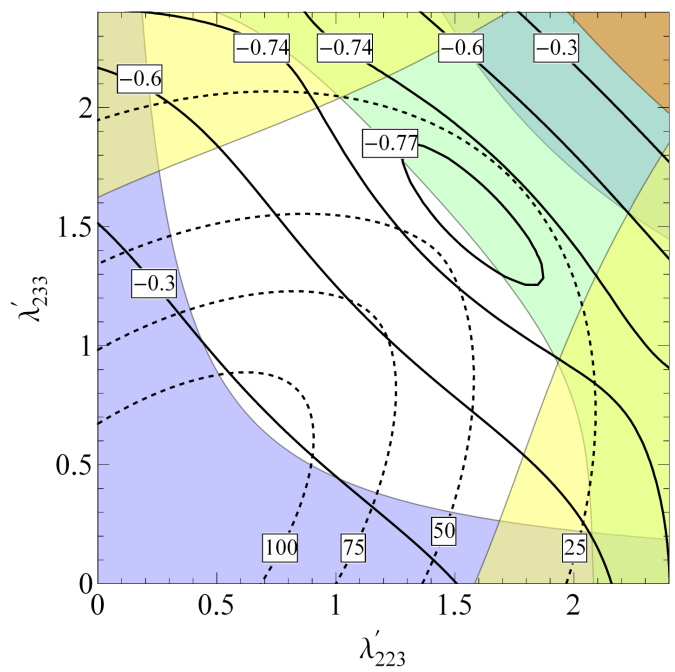

(a)

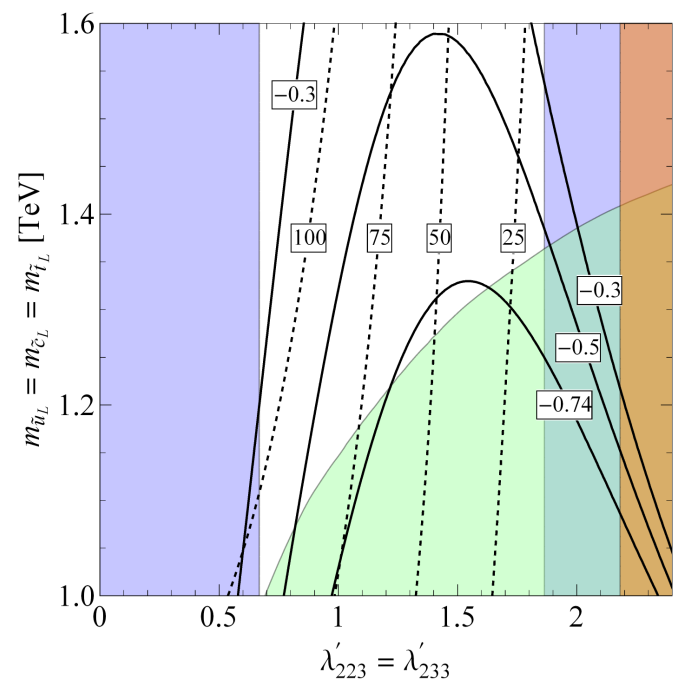

(c)

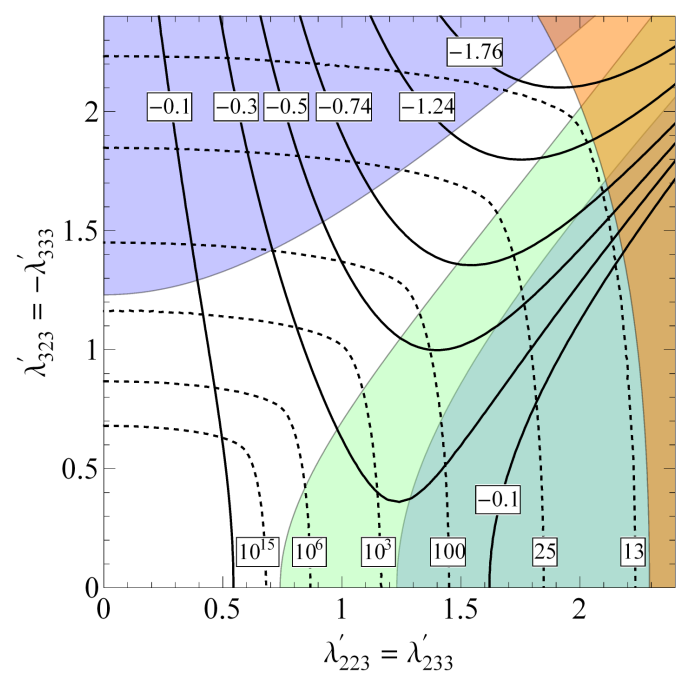

(b)

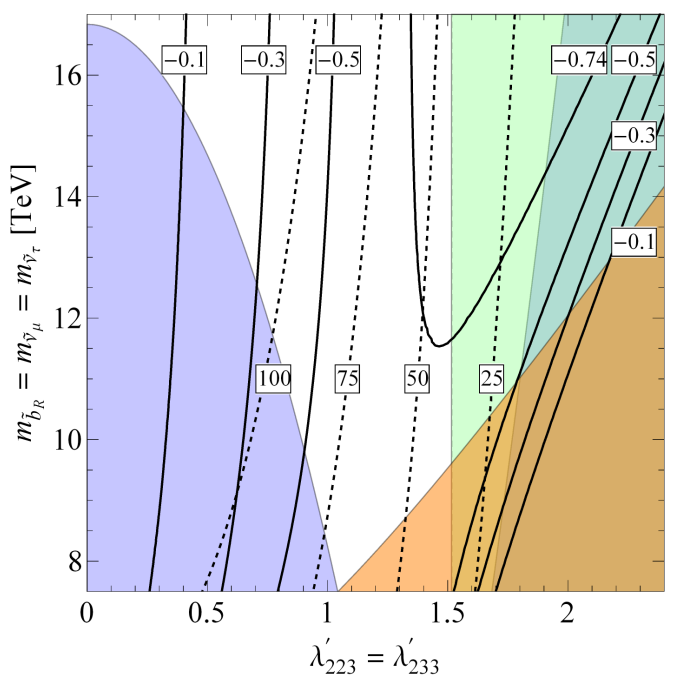

(d)

Figure 7.6: Four example figures showing solid contours of $C_{L L}^{\mu}$. For figure 7.6a, we set $\lambda_{323}^{\prime}=-\lambda_{333}^{\prime}=1.4, m_{\tilde{W}}=300 \mathrm{GeV}, m_{\tilde{u}_{L}}=m_{\tilde{c}_{L}}=m_{\tilde{t}_{L}}=1.3 \mathrm{TeV}$, and $m_{\tilde{b}_{R}}=m_{\tilde{\nu}_{\mu}}=m_{\tilde{\nu}_{\tau}}=13 \mathrm{TeV}$. For figure $7.6 \mathrm{~b}$, the masses are set to the same values as in figure $7.6 \mathrm{a}$. For figures $7.6 \mathrm{c}$ and $7.6 \mathrm{~d}, \lambda_{323}^{\prime}, \lambda_{333}^{\prime}$, and the masses not being varied are again set to the values used in figure 7.6a. Dashed contours show energy scales of Landau poles in TeV. Parameter space excluded by $\tau \rightarrow \mu \mu \mu$ is shown in yellow. Parameter space excluded by $B_{s}-\bar{B}_{s}$ mixing is shown in blue. Parameter space excluded by $B \rightarrow K^{(*)} \nu \bar{\nu}$ is shown in orange. Finally, parameter space excluded by direct LHC searches is shown in green. 
$\lambda_{223}^{\prime}<0$ and $\lambda_{233}^{\prime}<0$ or $\lambda_{323}^{\prime}<0$ and $\lambda_{333}^{\prime}>0$. For each of these plots, we have set the mass of the wino to be $300 \mathrm{GeV}$. We have also only considered mass degenerate left-handed up squarks and we have set the mass of the right-handed sbottom equal to the masses of the sneutrinos. Since we are primarily interested in the wino diagrams contribution to $C_{L L}^{\mu}$ we vary the parameters $\lambda_{223}^{\prime}$ and $\lambda_{233}^{\prime}$ in each of the plots. These are the only parameters varied in figure $7.6 \mathrm{a}$, while we also vary $\lambda_{323}^{\prime}$ and $\lambda_{333}^{\prime}$ in figure 7.6b, the masses of the left-handed up squarks in figure 7.6c, and masses of the right-handed sbottom and the sneutrinos in figure 7.6d.

Examining the plots, we observe the following features. First, it is difficult to generate very large values of $C_{L L}^{\mu}$ in this setup. We see that in all four plots only a small portion of the unexcluded parameter space has $C_{L L}^{\mu}<-0.74$, the upper limit of the $2 \sigma$ region capable of explaining the anomalies as stated in Ref. [240]. Indeed, in figures 7.6a, 7.6c, and 7.6d the largest value of $C_{L L}^{\mu}$ which can be generated and is not excluded is $\approx-0.77$. We see in figure $7.6 \mathrm{~b}$ that larger values of $C_{L L}^{\mu}$ can be generated but only if all four $\lambda^{\prime}$ couplings are taken large in magnitude. This leads to the second feature, large values of $C_{L L}^{\mu}$ necessarily imply low scale Landau poles. For each plot, the parameter region with $C_{L L}^{\mu}<-0.74$ also has a Landau pole at an energy scale $\lesssim 70 \mathrm{TeV}$. In fact, in figure $7.6 \mathrm{~b}$ we see that a portion of the otherwise unexcluded parameter space has Landau poles at energy scales less than the masses of the right-handed sbottom and sneutrinos. This region is thus also excluded. Third, notice that in figure $7.6 \mathrm{~b}$ the two regions excluded by $B_{s}-\bar{B}_{s}$ mixing do not converge, even for the largest values of the $\lambda^{\prime}$ couplings. This is an example of the cancellation amongst diagrams discussed in section 7.3.2. Fourth, as shown by figure 7.6c, the direct LHC search constraints require the masses of the left-handed up squarks to be $\gtrsim 1.4 \mathrm{TeV}$ if these particles decay only to $\mu b$. Smaller masses are allowed provided the 
left-handed up squarks decay to $\tau b$ as well. The final feature we wish to mention is that, as shown in figure 7.6d, the masses of the right-handed sbottom and sneutrinos need to be $\gtrsim 7.5 \mathrm{TeV}$. This demonstrates the smallest mass splitting between these particles and the left-handed up squarks that we can achieve in this setup.

\subsubsection{Additional remarks}

It is interesting to compare our results with those in Ref. [276]. There, the masses of all the sparticles are at the $\mathrm{TeV}$ scale and the negative contributions to $C_{L L}^{\mu}$ come from the four- $\lambda^{\prime}$ loop diagrams. In figure 7.7 , we show an example plot examining this parameter space. In this figure, we have set $\lambda_{323}^{\prime}=0.05, \lambda_{333}^{\prime}=-0.5, m_{\tilde{W}}=300 \mathrm{GeV}$, and $m_{\tilde{u}_{L}}=m_{\tilde{c}_{L}}=m_{\tilde{t}_{L}}=m_{\tilde{b}_{R}}=m_{\tilde{\nu}_{\mu}}=m_{\tilde{\nu}_{\tau}}=2 \mathrm{TeV}$. By setting the masses of the left-handed up squarks and right-handed bottom squark to $2 \mathrm{TeV}$ we avoid potential constraints from direct LHC searches. ${ }^{3}$ A new feature in this figure compared to the plots in figure 7.6 is that some of the parameter space is excluded by $Z$ decays to charge leptons. This type of constraint was not considered in Ref. [276]. Further, we see that achieving values of $C_{L L}^{\mu}<-0.74$ is still difficult in this setup as well. Also, the energy scales of the Landau poles are similar to those in figure 7.6. Finally, we note that by setting the masses of the sparticles to be of the same order, we are required to consider a hierarchical structure for the four $\lambda^{\prime}$ couplings under consideration. In our setup, the $\lambda^{\prime}$ couplings can be of the same magnitude but we are forced to consider a hierarchical structure for the sparticle masses.

To generate large values of $C_{L L}^{\mu}$ we have considered large values for the four parameters $\lambda_{223}^{\prime}, \lambda_{233}^{\prime}, \lambda_{323}^{\prime}$, and $\lambda_{333}^{\prime}$. Moreover, these couplings should also generate

\footnotetext{
${ }^{3}$ As shown in figure $7.6 \mathrm{c}$, the limits from pair produced squarks decaying to $\mu \mu b b$ saturate at $\sim 1.4 \mathrm{TeV}$. Additionally, pair produced right-handed sbottoms can also decay to the final state $\nu \nu b b$. The limits from this type of signature saturate at $\sim 1.1 \mathrm{TeV}[292]$.
} 


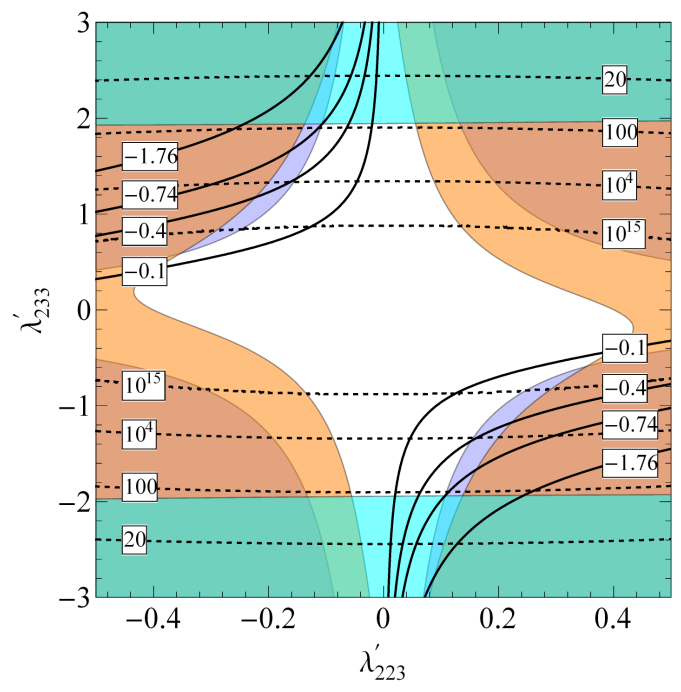

Figure 7.7: Example figure showing solid contours of $C_{L L}^{\mu}$ for parameter space similar to that considered in Ref. [276]. For this figure, we set $\lambda_{323}^{\prime}=0.05, \lambda_{333}^{\prime}=-0.5$, $m_{\tilde{W}}=300 \mathrm{GeV}$, and $m_{\tilde{u}_{L}}=m_{\tilde{c}_{L}}=m_{\tilde{t}_{L}}=m_{\tilde{b}_{R}}=m_{\tilde{\nu}_{\mu}}=m_{\tilde{\nu}_{\tau}}=2 \mathrm{TeV}$. Dashed contours show energy scales of Landau poles in TeV. Parameter space excluded by $B_{s}-\bar{B}_{s}$ mixing is shown in blue. Parameter space excluded by $B \rightarrow K^{(*)} \nu \bar{\nu}$ is shown in orange. Finally, parameter space excluded by $Z$ decays to charged leptons is shown in cyan.

contributions to $C_{L L}^{\tau}, C_{L L}^{\tau \mu}$, and $C_{L L}^{\mu \tau}$, each defined analogously to $C_{L L}^{\mu}$

$$
\mathcal{H}_{\mathrm{eff}}=-\frac{4 G_{F}}{\sqrt{2}} V_{t b} V_{t s}^{*} \frac{\alpha}{4 \pi} C_{L L}^{i j}\left(\bar{s} \gamma_{\alpha} P_{L} b\right)\left(\bar{\ell}_{i} \gamma^{\alpha} P_{L} \ell_{j}\right)+\text { h.c. }
$$

with $C_{L L}^{i} \equiv C_{L L}^{i i}$. These operators result in decays such as $B_{s} \rightarrow \mu \tau, B_{s} \rightarrow \tau \tau$, $B \rightarrow K^{(*)} \mu \tau$, and $B \rightarrow K^{(*)} \tau \tau$. Generically, each of these decays are not measured precisely enough (or at all) to cause any potential conflicts. For example, using just the effective Hamiltonian above, ${ }^{4}$ we find

$$
\operatorname{Br}\left(B_{s} \rightarrow \mu \tau\right)=5.4 \times 10^{-9}\left(\left|C_{L L}^{\mu \tau}\right|^{2}+\left|C_{L L}^{\tau \mu}\right|^{2}\right)
$$

\footnotetext{
${ }^{4}$ This is not quite right for the decay $B_{s} \rightarrow \tau \tau$ since there is also a Standard Model contribution given by $\operatorname{Br}\left(B_{s} \rightarrow \tau \tau\right)=(7.73 \pm 0.49) \times 10^{-7}[293,294]$.
} 
and

$$
\operatorname{Br}\left(B_{s} \rightarrow \tau \tau\right)=1.0 \times 10^{-8}\left|C_{L L}^{\tau}\right|^{2}
$$

However, we are unaware of any experimental bound on the former decay ${ }^{5}$ while the current experimental bound on the latter decay is $\operatorname{Br}\left(B_{s} \rightarrow \tau \tau\right)<6.8 \times 10^{-3}$ [297]. More details regarding the other two decays can be found in $[298,299]$.

The $\lambda^{\prime}$ couplings will also induce neutrino masses at the one loop level. Applying the general formula found in [19] to our setup, we find contributions to the neutrino mass matrix given by

$$
M_{i j}^{\nu}=\frac{3}{16 \pi^{2}} \lambda_{i 33}^{\prime} \lambda_{j l 3}^{\prime} m_{b}\left(\tilde{m}_{L R}^{d 2}\right)_{l 3} \frac{\log \left(m_{\tilde{b}_{R}}^{2} / m_{\tilde{d}_{L l}}^{2}\right)}{m_{\tilde{b}_{R}}^{2}-m_{\tilde{d}_{L l}}^{2}}+(i \leftrightarrow j)
$$

where $\tilde{m}_{L R}^{d 2}$ is the left-right sdown mass mixing matrix. In the normal RPVMSSM, this will generate neutrino masses that are far too large. As an example, consider the contribution to the $i=j=2$ entry from the case $l=3$. We then have that $\left(\tilde{m}_{L R}^{d 2}\right)_{33}=\left(A_{b}-\mu \tan \beta\right) m_{b}$. Taking $A_{b}-\mu \tan \beta=1 \mathrm{TeV}, m_{\tilde{b}_{R}}=13 \mathrm{TeV}, m_{\tilde{b}_{L}}=$ $1.3 \mathrm{TeV}$, and $\lambda_{233}^{\prime}=1.2$, we find $M_{22}^{\nu} \sim 10 \mathrm{keV}$, much larger than the $\sim 0.1 \mathrm{eV}$ limit on the neutrino mass scale. This potential difficulty was also pointed out in Ref. [279], who suggested $A_{b}$ and $\mu \tan \beta$ may cancel each other so that $\tilde{m}_{L R}^{d 2}$ is small. Another possibility mentioned in the same reference is that there may be additional unrelated contributions to the neutrino mass matrix which cancel those coming from equation 7.65. Alternatively, the situation can be improved by assuming a model of supersymmetry that possesses a $\mathrm{U}(1)_{R}$ symmetry identified with lepton number

\footnotetext{
${ }^{5} \mathrm{An}$ indirect bound can be placed on $\operatorname{Br}\left(B_{s} \rightarrow \mu \tau\right)$ by noting that this branching ratio is similar in size to $\operatorname{Br}\left(B^{+} \rightarrow K^{+} \mu \tau\right)$ [295] and that the Babar search [296] has provided the bound $\operatorname{Br}\left(B^{+} \rightarrow\right.$ $\left.K^{+} \mu \tau\right)<4.8 \times 10^{-5}$.
} 
$[172,175,186]$. These types of models, which feature the $\lambda^{\prime}$ couplings, assign different lepton number charges to the left and right-handed squarks. As a result, $\tilde{m}_{L R}^{d 2}$ vanishes in the limit that the $R$-symmetry is exact. However, the $R$-symmetry will be broken by at least anomaly mediation and this will generate contributions to $\tilde{m}_{L R}^{d 2}$ proportional to the gravitino mass. Parametrically we have

$$
\left(\tilde{m}_{L R}^{d 2}\right)_{33} \sim m_{3 / 2} \frac{m_{b}}{16 \pi^{2}}
$$

and this leads to

$$
M_{22}^{\nu} \sim 0.1 \mathrm{eV}\left(\frac{m_{3 / 2}}{1 \mathrm{GeV}}\right)
$$

where we have used the same values for the parameters as before. Thus, provided that the gravitino mass is lighter than $1 \mathrm{GeV}$, the model is safe from bounds on neutrino masses. Note that a gravitino in that mass range and stable on cosmological time scales can be problematic for cosmology as it can overclose the universe [300]. This can be solved by having a low reheat temperature or late entropy production.

Finally, we would like to briefly comment on the $R_{D^{(*)}}$ anomalies. These anomalies are the apparent enhancement of the ratio of branching ratios $R_{D}$ and $R_{D^{*}}$ defined in equation 7.5. Specifically, the current experimental values for these ratios are [258]

$R_{D}=0.403 \pm 0.040$ (stat) \pm 0.024 (syst) and $R_{D^{*}}=0.310 \pm 0.015$ (stat) \pm 0.008 (syst)

while the Standard Model predicts [301]

$$
R_{D}=0.299 \pm 0.003 \text { and } R_{D^{*}}=0.257 \pm 0.003
$$


When combined, these measurements represent an approximate $4 \sigma$ deviation away from the Standard Model [258]. The underlying quark transition $b \rightarrow c \ell \nu(\ell=e$, $\mu$, or $\tau$ ) can potentially occur by a tree level exchange of a right-handed sbottom with two $\lambda^{\prime}$ interactions. Indeed, the effect of these diagrams on the anomalies has previously been examined in the literature [277-279]. Following the analysis in [278], we find that our setup has essentially no impact on these anomalies because we have taken the mass of the right-handed sbottom to be large.

\subsection{Conclusion}

In this chapter, we examined the $b \rightarrow s \mu \mu$ anomalies within a supersymmetric framework with $R$-parity violation. Model independent analyses performed by different groups have shown that one way to explain these anomalies is to generate a negative contribution to the four-fermi operator $\left(\bar{s} \gamma_{\alpha} P_{L} b\right)\left(\bar{\mu} \gamma^{\alpha} P_{L} \mu\right)$. To do this, we considered the $R$-parity violating superpotential term $\lambda^{\prime} L Q D^{c}$ and studied many different diagrams. Initially, we examined a potentially relevant tree level diagram but found that it generates an effective four-fermi operator with an incorrect chirality structure. We then proceeded by studying multiple types of one loop diagrams. Specifically, we investigated the scenario in which the primary contribution is given by one loop box diagrams featuring a wino, with smaller contributions from one loop box diagrams featuring four $\lambda^{\prime}$ interactions. This led us to turning on the couplings $\lambda_{223}^{\prime}$, $\lambda_{233}^{\prime}, \lambda_{323}^{\prime}$, and $\lambda_{333}^{\prime}$ with $\lambda_{223}^{\prime} \lambda_{233}^{\prime}>0$ and $\lambda_{323}^{\prime} \lambda_{333}^{\prime}<0$. Additionally, this scenario requires a spectrum in which the masses of the wino and left-handed up squarks are of order $1 \mathrm{TeV}$ and the masses of the right-handed sbottom and sneutrinos are of order $10 \mathrm{TeV}$. We then studied many physical processes relevant to our parameters. Constraints were derived from various $\tau$ decays including $\tau \rightarrow \mu$ meson, $\tau \rightarrow \mu \gamma$, 
$\tau \rightarrow \mu \mu \mu$, and $\tau \rightarrow \mu e^{+} e^{-}$. Additional constraints were determined from $B_{s}-\bar{B}_{s}$ mixing, $B \rightarrow K^{(*)} \nu \bar{\nu}, Z$ decays to charged leptons, and direct LHC searches. Four example plots examining the parameter space were presented. These plots demonstrated that this setup can potentially explain the anomalies, although generating large contributions can be challenging. Moreover, to explain the anomalies, the four $\lambda^{\prime}$ couplings each need to be large and this necessarily leads to low scale Landau poles. We then compared our setup with a more traditional supersymmetric spectrum in which the masses of all the sparticles are at the $\mathrm{TeV}$ scale. Finally, we briefly discussed decays such as $B_{s} \rightarrow \mu \tau$ and $B_{s} \rightarrow \tau \tau$, contributions to the neutrino mass matrix, and how our model effects the anomalies related to the observables $R_{D^{(*)}}$.

To conclude, we will briefly summarize the different potential solutions to the $b \rightarrow s \mu \mu$ anomalies and the $R_{D^{(*)}}$ anomalies which involve $R$-parity violation. We have found a new region of parameter space capable of potentially explaining the $b \rightarrow s \mu \mu$ anomalies. This region is characterized by the wino and left-handed up squarks having masses of order $1 \mathrm{TeV}$ and the right-handed sbottom and sneutrinos having masses of order $10 \mathrm{TeV}$. The four couplings $\lambda_{223}^{\prime}, \lambda_{233}^{\prime}, \lambda_{323}^{\prime}$, and $\lambda_{333}^{\prime}$ are each of order 1 . For these parameters, $R_{D^{(*)}}$ receives no significant additional contributions, and thus this region of parameter space is unable to explain the anomalies associated with these observables. Crucially, this potential solution to the $b \rightarrow s \mu \mu$ anomalies relies on a light wino. If, on the other hand, the wino turns out to be heavy, then the $b \rightarrow s \mu \mu$ anomalies can still be explained as presented in [276]. This requires the masses of the left-handed up squarks, right-handed sbottom, and sneutrinos to be each of order $1 \mathrm{TeV}$. The same four $\lambda^{\prime}$ as in the light wino case are again non-zero but now $\lambda_{233}^{\prime}$ and $\lambda_{333}^{\prime}$ are of order 1 while $\lambda_{223}^{\prime}$ and $\lambda_{323}^{\prime}$ are much smaller. Although, as shown in figure 7.7 , totally explaining the $b \rightarrow s \mu \mu$ anomalies can still be challenging. 
These parameters can also lead to moderate contributions to $R_{D^{(*)}}$ [276]. Finally, it is possible to fully explain the anomalies in $R_{D^{(*)}}$ by making the mass of the righthanded sbottom less than $1 \mathrm{TeV}$ and only $\lambda_{333}^{\prime}$ large, but in this case it is now difficult to also explain the $b \rightarrow s \mu \mu$ anomalies [277-279]. 


\section{Chapter 8}

\section{The spontaneous $\mathbb{Z}_{2}$ breaking Twin}

\section{Higgs}

\subsection{Introduction}

Models of natural supersymmetry are examples of beyond the Standard Model (BSM) theories that stabilize the hierarchy between the electroweak and Planck scale. There are, of course, other examples of BSM physics that can potentially stabilize this hierarchy. To this end, most BSM models introduce partner particles that cancel the quadratically divergent corrections to the Higgs mass. These partners are generally assumed to be charged under the Standard Model gauge groups. More specifically, the partner particle for the top quark is assumed to be charged under SM colour. Unfortunately, the lack of discovery of new particles during run 1 and run 2 of the LHC has put strong constraints on these partners and further accentuates the little hierarchy problem [302]. Thus, for the final project presented in this thesis, we turn away from supersymmetry and consider other ways of realizing a natural theory while at the same time avoiding constraints coming from the LHC. 
One way to do this is neutral naturalness, the idea that partners are not charged under the SM gauge groups, or, at the very least, not charged under SM colour. Perhaps the best example of this is Twin Higgs [303] (see [304] for another neutral naturalness model known as folded supersymmetry and [305-322] for other related work on neutral naturalness models). This model rests on a global SU(4) which is broken spontaneously to $\mathrm{SU}(3)$ at a scale $f$, leading to a set of Goldstone bosons. The $\mathrm{SU}(4)$ is explicitly broken by gauging a $\mathrm{SU}(2)_{A} \times \mathrm{SU}(2)_{B}$ subgroup (with $\mathrm{SU}(2)_{A}$ being identified with the $\mathrm{SM} \mathrm{SU}(2)_{\mathrm{L}}$ and $\mathrm{SU}(2)_{B}$ a similar symmetry of a mirror sector) and by adding Yukawa couplings. In principle, this breaking would give a mass of order $f$ to the Goldstone bosons. Remarkably, imposing a $\mathbb{Z}_{2}$ symmetry between the two sectors ensures that the theory is still $\mathrm{SU}(4)$ invariant at the quadratic level, leading to a light pseudo-Goldstone Higgs. A soft $\mathbb{Z}_{2}$ breaking is however needed to obtain a hierarchy of vacuum expectation values between the Standard Model Higgs and the mirror sector Higgs [303,305]. ${ }^{1}$

Despite its success, even the Twin Higgs is not free from tuning. A moderate amount of tuning between the $\mathbb{Z}_{2}$ and the $\mathrm{SU}(4)$ breaking sectors is needed to push the cutoff beyond experimental constraints. Various attempts at addressing this issue can be found in the literature. Reference [323] tries to do so in the context of a two Higgs doublet model with misaligned vevs. In [324], the issue is addressed in a supersymmetric UV completion by introducing Dirac gauginos [162]. Finally, [325] also addresses the supersymmetric completion, but by forcing $\tan \beta=1$ in the mirror sector. Both of these models try to remove the D-term quartics which are a source of tuning in supersymmetric versions of the Twin Higgs. One thing all of these models have in common is an explicit $\mathbb{Z}_{2}$ breaking.

In this chapter, we propose a novel approach to improving the tuning in Twin

\footnotetext{
${ }^{1}$ See section 8.2 .1 for more details.
} 
Higgs, which is based on spontaneous breaking of the $\mathbb{Z}_{2}$ symmetry. The proposed model includes two Higgses in the fundamental representation of a SU(4) global symmetry. As in the original Twin Higgs model, a $\mathrm{SU}(2)_{A} \times \mathrm{SU}(2)_{B}$ subgroup is gauged and a $\mathbb{Z}_{2}$ symmetry is imposed between the two sectors. We take the vacuum of the first Higgs to preserve $\mathbb{Z}_{2}$, while the other breaks it spontaneously. A bilinear term containing the two Higgses is added (similar to the $B_{\mu}$ term of the MSSM) and the $\mathbb{Z}_{2}$ breaking is transmitted from the broken to the unbroken sector. This naturally produces a hierarchy between the vevs of the SM sector Higgses and those of the mirror sector. The $B_{\mu}$ term acts as an effective tadpole and no explicit $\mathbb{Z}_{2}$ breaking is necessary. The presence of this effective tadpole and feedback between the two Higgses lead to less tuning than the original Twin Higgs. The resulting Higgs boson is naturally very SM-like.

This chapter is organized as follows. We begin by summarizing the original Twin Higgs model to isolate the origin of the tuning and obtain results that will make comparisons with our model easier. Our model is then presented in detail. An analysis of the radiative corrections follows. A detailed analysis of the tuning of the model compared to the original Twin Higgs is then performed. Finally, a few concluding remarks including possible UV completions are presented.

\subsection{The model}

\subsubsection{The original Twin Higgs}

To put the problem our model attempts to solve in context and to establish our notation, we summarize the Twin Higgs model. We follow closely [303]. Assume a complex scalar field $H$ which transforms in the fundamental representation of a global 
$\mathrm{SU}(4)$. Its potential can be written as

$$
V_{\mathrm{SU}(4)}(H)=-\mu^{2} H^{\dagger} H+\lambda\left(H^{\dagger} H\right)^{2}
$$

The potential exhibits spontaneous symmetry breaking of $\mathrm{SU}(4) \rightarrow \mathrm{SU}(3)$. This leads to $\langle H\rangle \equiv f=\mu / \sqrt{2 \lambda}$ and 7 Goldstone bosons. The SM-like Higgs doublet is associated with 4 of these Goldstone bosons and is at this stage massless.

The $\mathrm{SU}(4)$ is then explicitly broken by gauging one of its $\mathrm{SU}(2)_{A} \times \mathrm{SU}(2)_{B}$ subgroups. The field $H$ is now divided into fundamental representations of $\mathrm{SU}(2)_{A}$ and $\mathrm{SU}(2)_{B}$ as $H=\left(H_{A}, H_{B}\right)$. The $A$ sector is conventionally associated with the Standard Model and the $B$ sector with the mirror sector. The leading correction to the potential introduced by gauging the $\mathrm{SU}(2)$ 's is

$$
\Delta V(H)=\frac{9 g_{A}^{2} \Lambda^{2}}{64 \pi^{2}} H_{A}^{\dagger} H_{A}+\frac{9 g_{B}^{2} \Lambda^{2}}{64 \pi^{2}} H_{B}^{\dagger} H_{B}
$$

where $g_{A}$ and $g_{B}$ are the gauge coupling constants of $\mathrm{SU}(2)_{A}$ and $\mathrm{SU}(2)_{B}$ respectively and $\Lambda$ is the cutoff of the theory. If a $\mathbb{Z}_{2}$ symmetry is imposed between the $A$ and $B$ sector, $g_{A}=g_{B} \equiv g$ and $\Delta V(H)$ accidentally respects the original $\mathrm{SU}(4)$ symmetry. The Goldstone bosons therefore do not acquire any mass from equation 8.2. Alternatively, one can then consider equation 8.2 as simply a correction to $\mu^{2}$. $\mathrm{SU}(4)$ will however be broken by terms of the form $\kappa\left(\left|H_{A}\right|^{4}+\left|H_{B}\right|^{4}\right)$, where $\kappa$ is of order $\left(g^{2} / 16 \pi^{2}\right) \log (\Lambda / f)$. These logarithmic divergences can be reabsorbed in $\lambda$ and a $\mathrm{SU}(4)$ breaking potential of the form

$$
V_{\text {SU(4) }}(H)=\alpha H_{A}^{\dagger} H_{A} H_{B}^{\dagger} H_{B}
$$


A similar story holds for the top Yukawa coupling. A $\mathbb{Z}_{2}$ symmetry is imposed on this sector by adding a 'mirror top' which is not charged under the SM groups, but which couples to $H_{B}$ in exactly the same way in which the SM top couples to $H_{A}$.

The total potential at this point is the sum of equations 8.1 and 8.3. The end result is that, of the original 7 Goldstone bosons, 6 will remain massless and be eaten by massive gauge bosons and the one left over will be a light pseudo-Goldstone boson that can be associated with the $125 \mathrm{GeV}$ Higgs. Since $\alpha$ is the only term in the potential that breaks $\mathrm{SU}(4)$, it can naturally be smaller than $\lambda$, which is what we assume. This ensures that the Higgs remains light even for relatively large $f$.

The symmetry breaking structure is controlled by the sign of $\alpha$ [305]. If $\alpha<0$, the minimum preserves $\mathbb{Z}_{2}$ and $\left\langle H_{A}\right\rangle=\left\langle H_{B}\right\rangle=\mu / \sqrt{4 \lambda+\alpha} \approx 174 \mathrm{GeV}$. This is the sign of $\alpha$ assumed in the original Twin Higgs model. The fact that $\left\langle H_{A}\right\rangle=\left\langle H_{B}\right\rangle$ leads to the Standard Model Higgs strongly mixing with the mirror sector Higgs and results in large deviations of the Higgs measurements [305]. It also means that $f$ is only slightly above the electroweak scale. The energy scale $\sim 4 \pi f$, at which new physics needs to appear to avoid fine-tuning, is then not much larger than in the Standard Model. These issues are easily resolved by aligning the vev closer to the $B$ sector, thereby allowing for a larger $f$ while preserving $\left\langle H_{A}\right\rangle=174 \mathrm{GeV}$. This can be done via an explicit soft $\mathbb{Z}_{2}$ breaking potential of the form

$$
V_{\mathbb{Z}_{2}}(H)=\Delta m^{2} H_{A}^{\dagger} H_{A} .
$$

The parameter $\Delta m^{2}$ can naturally be small as it is the only term that explicitly breaks $\mathbb{Z}_{2}$. The potential can be minimized by using the following parametrization of 
the relevant parts of $H$

$$
H=f\left(\begin{array}{c}
0 \\
\sin \theta \\
0 \\
\cos \theta
\end{array}\right)
$$

with $\theta$ being $\pi / 4$ when $\Delta m^{2}$ is 0 . The potential is minimized for a value of $f$ of

$$
f^{2}=\frac{2 \mu^{2}-\Delta m^{2}}{4 \lambda+\alpha}
$$

while minimizing the potential with respect to $\theta$ gives the following equation

$$
\alpha f^{4} \sin 4 \theta+4 \Delta m^{2} f^{2} \sin \theta \cos \theta=0 .
$$

This equation only yields non-zero $\theta$ for $\Delta m^{2}$ below a maximal value. Thus, we define $\Delta m_{\max }^{2}$ as the largest value of $\Delta m^{2}$ for which there is still electroweak symmetry breaking in the $A$ sector. It can be found by rewriting equation 8.7 as

$$
F_{1}(\theta) \equiv \frac{1}{4} \frac{\sin 4 \theta}{\sin \theta \cos \theta}=\frac{\Delta m^{2}}{\left(-\alpha f^{2}\right)} \approx \frac{\Delta m^{2}}{\Delta m_{\max }^{2}}
$$

where the last relation holds in the limit of small $\alpha$ and $\Delta m_{\max }^{2}$ is given by the exact relation

$$
\Delta m_{\max }^{2}=-\frac{\alpha \mu^{2}}{2 \lambda}
$$

The solution to equation 8.7 is

$$
\sin ^{2} \theta=\frac{v^{2}}{f^{2}}=\frac{1}{2}\left(1-\frac{\Delta m^{2}}{\left(-\alpha f^{2}\right)}\right) \approx \frac{1}{2}\left(1-\frac{\Delta m^{2}}{\Delta m_{\max }^{2}}\right)
$$


where $v$ is the SM Higgs vev of $174 \mathrm{GeV}$. Requesting a large $f$ implies a tuning between the $\mathrm{SU}(4)$ breaking and the $\mathbb{Z}_{2}$ breaking potentials. This is reflected in equation 8.10 by the last term on the right needing to be close to 1 .

Alternatively, one can take $\alpha>0$. The $\mathbb{Z}_{2}$ symmetry is then spontaneously broken and the system falls in one of the two minima at $\left\langle H_{A}\right\rangle=\mu / \sqrt{2 \lambda}$ and $\left\langle H_{B}\right\rangle=0$ or $\left\langle H_{A}\right\rangle=0$ and $\left\langle H_{B}\right\rangle=\mu / \sqrt{2 \lambda}$. However, the vev must be taken to fall in the SM sector and this leads to a massless mirror sector. This proves to be unviable for cosmological reasons [305]. The potential must then be modified in a way similar to equation 8.4 to prevent the minimum from being in one sector only. Unfortunately, a quick inspection shows that no term that only breaks $\mathbb{Z}_{2}$ softly and respects gauge invariance can do so. The term of equation 8.4 does not solve this problem, as equation 8.7 is satisfied by a $\theta$ of 0 for all values of $\Delta m^{2}$. The case of $\alpha>0$ therefore poses serious issues.

\subsubsection{Spontaneous $\mathbb{Z}_{2}$ breaking}

In the last section, part of the problem in the $\alpha>0$ case was that $H$ was the only scalar with gauge charges. This forced all terms in the potential to be an even power of $H$ and forbade tadpole terms, which could have potentially prevented the vev from falling in one sector only. The inclusion of a second Higgs field can solve this problem by including a term linear in both fields which acts as an effective tadpole for $H$ (see [326] for a similar idea in a context unrelated to $\mathbb{Z}_{2}$ breaking or the Twin Higgs). In addition, the $\mathbb{Z}_{2}$ breaking soft term for $\alpha<0$ also needed to be quadratic in $H$. It is possible that a similar term with a lower power of $H$ could potentially produce the same hierarchy of vevs while requiring less tuning. Again, a term linear in $H$ and another Higgs can do this. Taking these considerations into account, our 
model includes two fundamental representations of $\mathrm{SU}(4), H_{1}=\left(H_{1 A}, H_{1 B}\right)$ and $H_{2}=\left(H_{2 A}, H_{2 B}\right)$, that are gauged as in Twin Higgs and which interact with each other to create a hierarchy of vevs. We take the minimum of $H_{1}$ to preserve $\mathbb{Z}_{2}$ and that of $H_{2}$ to break it. It is the interaction between $H_{1}$ and $H_{2}$ that transmits the $\mathbb{Z}_{2}$ breaking to $H_{1}$ and there is no need for an explicit $\mathbb{Z}_{2}$ breaking. We explain the finer details below.

\subsubsection{Potential and vevs}

As a starting point, we write down the potential for $H_{1}$ by itself

$$
V_{H_{1}}\left(H_{1}\right)=-\mu_{1}^{2} H_{1}^{\dagger} H_{1}+\lambda_{1}\left(H_{1}^{\dagger} H_{1}\right)^{2}+\alpha_{1} H_{1 A}^{\dagger} H_{1 A} H_{1 B}^{\dagger} H_{1 B}
$$

and assume $\alpha_{1}<0$, which means that the vacuum preserves $\mathbb{Z}_{2}$. At this point, the pseudo-Goldstone boson from $H_{1}$ corresponds to the angular mode and is an equal admixture of the components of $H_{1 A}$ and $H_{1 B}$. Similarly, we write a potential for $H_{2}$ by itself

$$
V_{H_{2}}\left(H_{2}\right)=-\mu_{2}^{2} H_{2}^{\dagger} H_{2}+\lambda_{2}\left(H_{2}^{\dagger} H_{2}\right)^{2}+\alpha_{2} H_{2 A}^{\dagger} H_{2 A} H_{2 B}^{\dagger} H_{2 B}
$$

and this time with $\alpha_{2}>0$, meaning that the vacuum breaks $\mathbb{Z}_{2}$ in this case. We take the vev to fall in the $B$ sector by convention, as the vev falling in the other sector would just mean a relabelling of $B$ as the SM and $A$ as the mirror sector. The pseudo-Goldstone boson again corresponds to the angular mode. This time however, the position of the minimum means that the pseudo-Goldstone boson is purely a component of $H_{2 A}$.

The interaction between these two fields is then codified by the following potential

$$
V_{H_{1} H_{2}}\left(H_{1}, H_{2}\right)=-B_{\mu} H_{1}^{\dagger} H_{2}+\text { h.c. }
$$




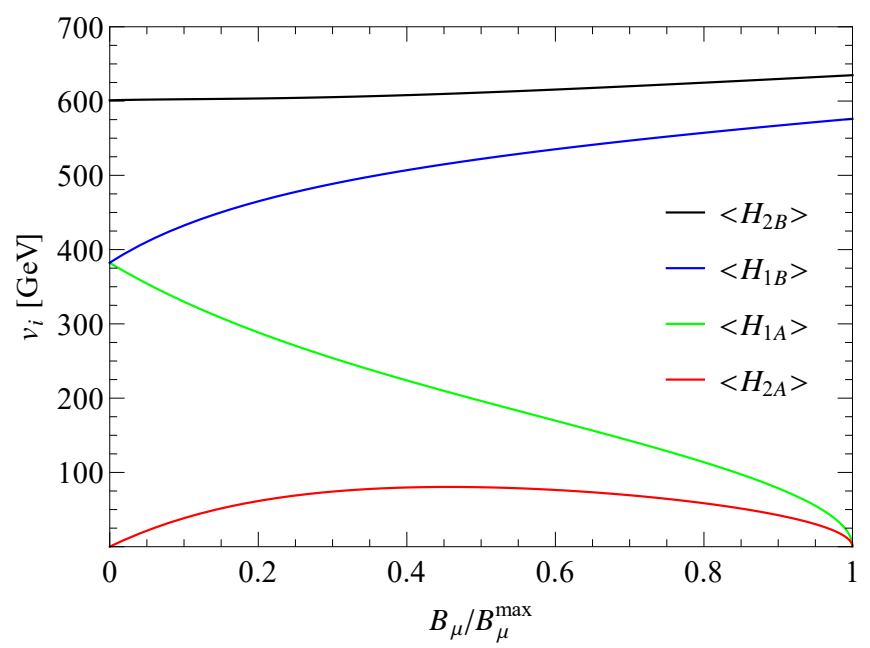

Figure 8.1: Example of the different vevs as a function of $B_{\mu} / B_{\mu}^{\max }$. The parameters are $\mu_{1}=750 \mathrm{GeV}, \mu_{2}=850 \mathrm{GeV}, \alpha_{1}=-0.15, \alpha_{2}=0.2$, and $\lambda_{1}=\lambda_{2}=1$.

We note that it is technically natural to have $B_{\mu}$ small as it breaks a Peccei-Quinn symmetry. For $B_{\mu}$ small and greater than zero, equation 8.13 serves essentially two purposes. First, the part $H_{1 B}^{\dagger} H_{2 B}$ serves as an effective tadpole for $H_{1 B}$. It therefore pushes the vev of $H_{1}$ toward the $B$ sector, as desired. Second, the part $H_{1 A}^{\dagger} H_{2 A}$ serves as an effective tadpole for $H_{2 A}$. It accordingly provides a small positive $A$ component to the vev of $H_{2}$. As $B_{\mu}$ increases, non-linear effects and feedback between the different terms become important. An example of the different vevs is shown in figure 8.1.

\subsubsection{Small $\alpha_{i}$ 's approximation}

To gain a better understanding of the interactions between $H_{1}$ and $H_{2}$, we decompose them in a similar way to equation 8.5 and take the limit of small $\alpha_{i}$ 's. As will be made clear in equation $8.19, B_{\mu}$ will be a factor of $\alpha_{1} / \lambda_{1}$ smaller than the $\mu_{i}^{2}$ 's in the physically viable and natural region of parameter space. We therefore assume it to be small. In general, all approximations will be valid up to $\mathcal{O}\left(\alpha_{i} / \lambda_{i}\right)$. The decomposition 
of the Higgses is

$$
H_{1}=f_{1}\left(\begin{array}{c}
0 \\
\sin \theta_{1} \\
0 \\
\cos \theta_{1}
\end{array}\right) \quad H_{2}=f_{2}\left(\begin{array}{c}
0 \\
\sin \theta_{2} \\
0 \\
\cos \theta_{2}
\end{array}\right)
$$

where $f_{1} \approx \mu_{1} / \sqrt{2 \lambda_{1}}$ and $f_{2} \approx \mu_{2} / \sqrt{2 \lambda_{2}}$. The minimization of the potential with respect to the angles leads to the set of equations

$$
\begin{aligned}
& \alpha_{1} f_{1}^{4} \sin 4 \theta_{1}+4 B_{\mu} f_{1} f_{2} \sin \left(\theta_{1}-\theta_{2}\right)=0 \\
& \alpha_{2} f_{2}^{4} \sin 4 \theta_{2}-4 B_{\mu} f_{1} f_{2} \sin \left(\theta_{1}-\theta_{2}\right)=0 .
\end{aligned}
$$

When $B_{\mu}=0$, the minimum is located at $\theta_{1}=\pi / 4$ and $\theta_{2}=0$. In the general case, adding both equations leads to

$$
\sin 4 \theta_{2}=\Omega \sin 4 \theta_{1}
$$

where $\Omega$ is a constant in the small $\alpha$ approximation and is defined by

$$
\Omega \equiv-\frac{\alpha_{1}}{\alpha_{2}}\left(\frac{f_{1}}{f_{2}}\right)^{4}
$$

First, consider $\Omega<1$. Increasing $B_{\mu}$ will make $\theta_{1}$ pass from $\pi / 4$ to 0 . The angle $\theta_{2}$ starts by increasing but decreases once $\theta_{1}$ drops below $\pi / 8$. Eventually, both angles settle at 0 . When $\Omega>1$, this behaviour is reversed. Increasing $B_{\mu}$ will make $\theta_{2}$ pass from 0 to $\pi / 4$. The angle $\theta_{1}$ decreases until $\theta_{2}$ reaches $\pi / 8$, but increases afterward. Both angles ultimately settle to $\pi / 4$. This behaviour is not bad in itself as it can still lead to a small hierarchy, but obtaining a large one proves to be impossible. Taking 


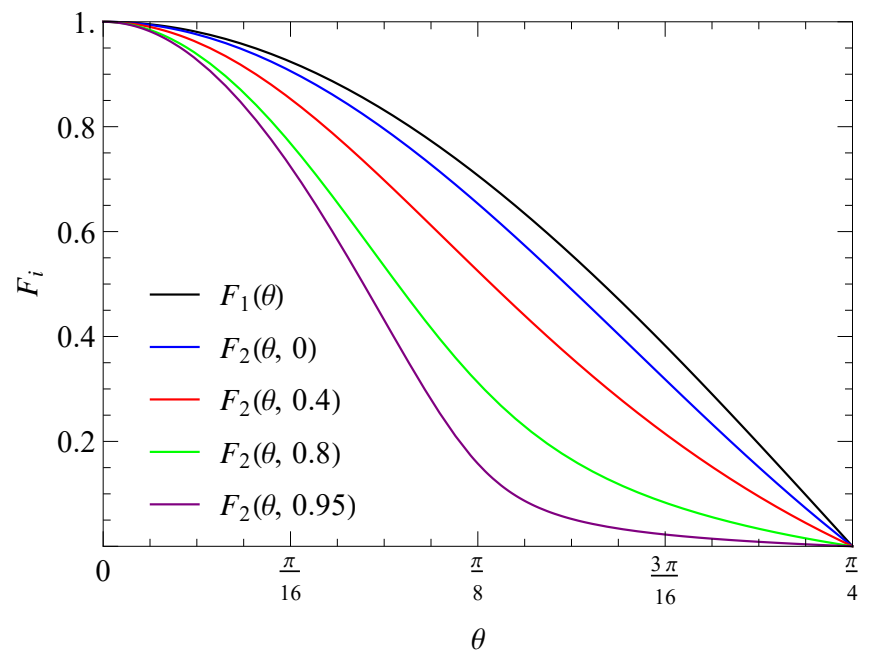

Figure 8.2: $F_{1}(\theta)$ and $F_{2}(\theta, \Omega)$ for different values of $\Omega$.

these considerations into account, we focus on the domain where $\Omega<1$.

Analogous to the Twin Higgs case, we define $B_{\mu}^{\max }$ as the largest value of $B_{\mu}$ for which there is still electroweak symmetry breaking in the $A$ sector. The first line of equation 8.15 can then be rewritten as

$$
F_{2}\left(\theta_{1}, \Omega\right) \equiv \frac{(1-\Omega)}{4} \frac{\sin 4 \theta_{1}}{\sin \left(\theta_{1}-\theta_{2}\right)}=\frac{B_{\mu}}{\left(-\frac{\alpha_{1} f_{1}^{3}}{f_{2}(1-\Omega)}\right)} \approx \frac{B_{\mu}}{B_{\mu}^{\max }}
$$

where $\theta_{2}$ is related to $\theta_{1}$ by equation 8.16 . In the small $\alpha_{i}{ }^{\prime}$ s approximation, $B_{\mu}^{\max }$ is then

$$
B_{\mu}^{\max } \approx-\frac{\alpha_{1} f_{1}^{3}}{f_{2}(1-\Omega)}
$$

While it is hard to solve equation 8.18 for $\theta_{1}$, it is easy to see that small values of $\theta_{1}$ require $B_{\mu}$ to be close to $B_{\mu}^{\max }$. This is similar to the Twin Higgs case where $\Delta m^{2}$ needed to be close to $\Delta m_{\max }^{2}$ to obtain a small ratio of vevs.

We can compare the two theories by looking at $F_{1}(\theta)$ and $F_{2}(\theta, \Omega)$ which are plotted in figure 8.2 for different values of $\Omega$ between 0 and 1 . When $0<\theta<\pi / 4$, 
$F_{2}(\theta, \Omega)$ is always smaller than $F_{1}(\theta)$. This means that, for the same angle, our model does not require $B_{\mu}$ as close to $B_{\mu}^{\max }$ as the Twin Higgs requires $\Delta m^{2}$ close to $\Delta m_{\max }^{2}$. This translates to less tuning. In contrast to the Twin Higgs, one must keep in mind that for our model $\left\langle H_{1 A}\right\rangle<v=174 \mathrm{GeV}$, as it is a two Higgs doublet model. As avoiding large tuning requires new physics near $\sim 4 \pi f_{1}$, this suggests that for equivalent tuning and cutoff one must choose $\theta_{1}$ smaller than the equivalent angle in Twin Higgs. Fortunately, our model naturally leads to $\left\langle H_{1 A}\right\rangle$ considerably larger than $\left\langle H_{2 A}\right\rangle$. Thus, the difference is small and the argument about tuning remains valid.

Further insight can be obtained by taking the small $\theta_{1}$ limit of equation 8.18

$$
\theta_{1}^{2} \approx \frac{3}{8} \frac{\left(B_{\mu}^{\max }-B_{\mu}\right)}{\left(B_{\mu}^{\max }+g(\Omega) B_{\mu}\right)} \stackrel{B_{\mu} \rightarrow B_{\mu}^{\max }}{\sim} \frac{3}{8(1+g(\Omega))}\left(1-\frac{B_{\mu}}{B_{\mu}^{\max }}\right)
$$

where

$$
g(\Omega) \equiv \frac{1}{16}\left(15 \Omega^{2}+18 \Omega-1\right)
$$

As mentioned above, a more appropriate quantity to make the comparison with the Twin Higgs is

$$
\begin{aligned}
\frac{v^{2}}{f_{1}^{2}} & \sim \frac{3}{8(1+g(\Omega))}\left(1+\left(-\frac{\alpha_{2}}{\alpha_{1}}\right)^{-1 / 2} \Omega^{3 / 2}\right)\left(1-\frac{B_{\mu}}{B_{\mu}^{\max }}\right) \\
& \equiv C\left(-\alpha_{2} / \alpha_{1}, \Omega\right)\left(1-\frac{B_{\mu}}{B_{\mu}^{\max }}\right) .
\end{aligned}
$$

This is to be compared to equation 8.10 which has a similar structure but with $C\left(-\alpha_{2} / \alpha_{1}, \Omega\right)$ replaced by $1 / 2$. Figure 8.3 shows $C\left(-\alpha_{2} / \alpha_{1}, \Omega\right)$ as a function of $\Omega$ for fixed values of $-\alpha_{2} / \alpha_{1}$. We see that, unless $-\alpha_{2} / \alpha_{1}$ is very small, $C\left(-\alpha_{2} / \alpha_{1}, \Omega\right)$ is smaller than $1 / 2$ for $\Omega$ in the whole range of 0 to 1 . This shows that our model 


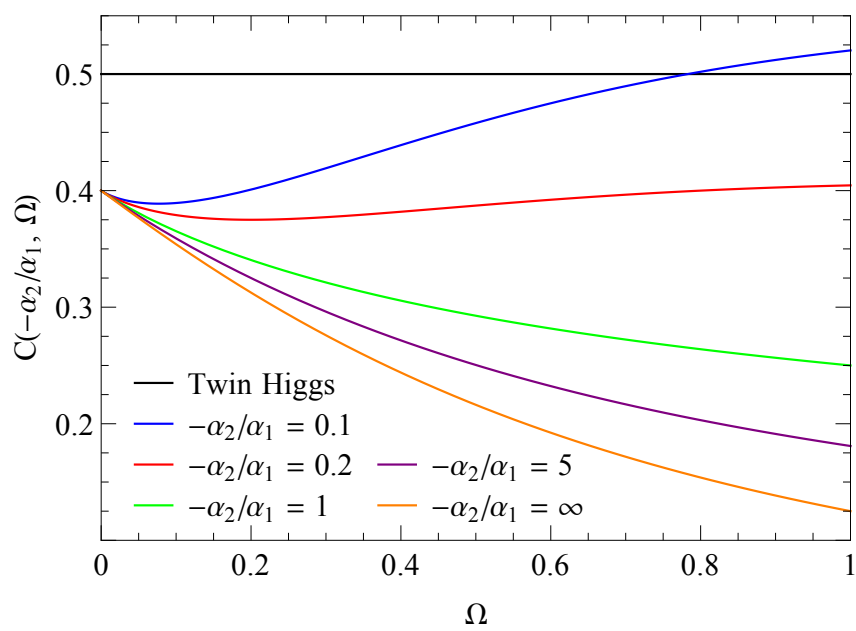

Figure 8.3: $C\left(-\alpha_{2} / \alpha_{1}, \Omega\right)$ for various values for $-\alpha_{2} / \alpha_{1}$. Also shown is the corresponding value for Twin Higgs.

can easily obtain the same cutoff as the Twin Higgs for less tuning.

The improvement in the tuning can ultimately be attributed to two sources. First, we can look at the limit of small $\Omega$, which means that $\theta_{2}$ is also small. This limit means that $H_{2}$ only serves as an effective tadpole and does not mix with $H_{1}$. The fact that the symmetry breaking is induced by an effective tadpole translates to equation 8.18 missing the factor of $\cos \theta$ present in equation 8.8. This by itself is enough to ensure that $F_{2}(\theta, \Omega)$ be smaller than $F_{1}(\theta)$. Second, there is considerable feedback between $H_{1}$ and $H_{2}$ when $\Omega$ is close to 1 . This translates to $\theta_{2}$ and $1-\Omega$ appearing in equation 8.18. The presence of these terms further decreases $F_{2}(\theta, \Omega)$, as is clearly shown in figure 8.2. Obviously, taking $\Omega$ close to 1 is a tuning in itself, though certainly not large enough to spoil our results, and we take this into account in section 8.3. 


\subsubsection{Additional properties}

A few additional properties of the model are worth mentioning. The first one is that the behaviour of figure 8.1 can differ outside of the region of parameter space considered up to now. The case of $\Omega>1$ mentioned above is an example. Even when $\Omega<1$, the vevs can act differently if the $\alpha_{i}$ 's or $B_{\mu}$ are large. In particular, it is possible to choose parameters such that the vevs of the $A$ sector start like those of figure 8.1 but fail to reach 0 . It is also possible for the vevs of the $A$ sector to be 0 for an interval of $B_{\mu}$ but then become non-zero again for very large $B_{\mu}$. We therefore define more precisely $B_{\mu}^{\max }$ as the smallest positive value of $B_{\mu}$ for which the vevs of the $A$ sector are zero. Fortunately, a sufficient condition for $B_{\mu}^{\max }$ to exist, which is that the vevs of the $A$ sector settle to 0 for large $B_{\mu}$, is easily satisfied and given by

$$
\frac{\alpha_{1}}{\lambda_{1}}+\frac{\alpha_{2}}{\lambda_{2}}+\frac{\alpha_{1} \alpha_{2}}{2 \lambda_{1} \lambda_{2}}>0
$$

When this relation is close to being satisfied but not quite, it is possible that the system falls in the scenario where the vevs in the $A$ sector are 0 for an interval but become positive again for large $B_{\mu}$. This relation comes from looking at the limit of large $B_{\mu}$, where the $\mu_{i}^{2}$ 's can be ignored. In this case, setting $\alpha_{2}$ to 0 will result in the potential being minimized for both $\theta_{i}$ 's being $\pi / 4$. Increasing $\alpha_{2}$ while keeping the other parameters fixed will cause both angles to eventually move toward 0 . The angles will settle to 0 (which is always an extremum) when this point becomes a minimum, which happens when equation 8.23 is satisfied. The vevs of the $A$ sector will then be 0 for large enough $B_{\mu}$ and it is therefore sufficient for $B_{\mu}^{\max }$ to exist.

Also of importance is that when $B_{\mu}=0$ the pseudo-Goldstone boson from $H_{1}$ is an equal combination of the $A$ and $B$ sector, while the one from $H_{2}$ is purely in 
the $A$ sector. One would then expect that turning on $B_{\mu}$ would cause the resulting light pseudo-Goldstone boson to be more $A$-like than in the equivalent case for Twin Higgs. This turns out to be the case. To see this, we decompose the lightest pseudoGoldstone as

$$
h=a h_{1 A}+b h_{2 A}+c h_{1 B}+d h_{2 B}
$$

where $h_{1 A}$ is defined via $H_{1 A}^{0}=\left(v_{1 A}+\left(h_{1 A}+i A_{1 A}\right) / \sqrt{2}\right)$ and identically for the other $h_{i}$ 's. The parameter $\Theta_{B} \equiv c^{2}+d^{2}$ represents a measure of how much the Higgs is $B$-like. A similar quantity can easily be defined for the Twin Higgs. The comparison for both models can be seen in figure 8.4. Note that the pseudo-Goldstone is most $A$ like for large mixing between $H_{1}$ and $H_{2}$. The price to pay for this is that constraints akin to those in the usual two Higgs doublet model become important. Fortunately, these constraints can easily be avoided, as the model naturally leads to a hierarchy between the vevs in the $A$ sector and fairly little mixing with mirror sector Higgses. Generally speaking, this means that our model will be better at avoiding constraints on Higgs couplings, though a full study of this is beyond the scope of this chapter.

The particle spectrum in the $A$ sector is the usual two Higgs doublet model one. Generically speaking, creating a small hierarchy will push the masses of the heavier Higgses up for a fixed value of the lightest Higgs. The constraints from heavy scalar searches can therefore be easily avoided.

Another point worth mentioning is that the potential we wrote down does not contain all possible $\mathbb{Z}_{2}$ preserving terms. We verified that these extra terms do not affect the qualitative behaviour of the system, as long as they are not much bigger than the terms already included. Even small explicit $\mathbb{Z}_{2}$ breaking terms do not affect the qualitative behaviour of the system. Unless stated otherwise, such terms will be ignored from now on to avoid obscuring the analysis. 


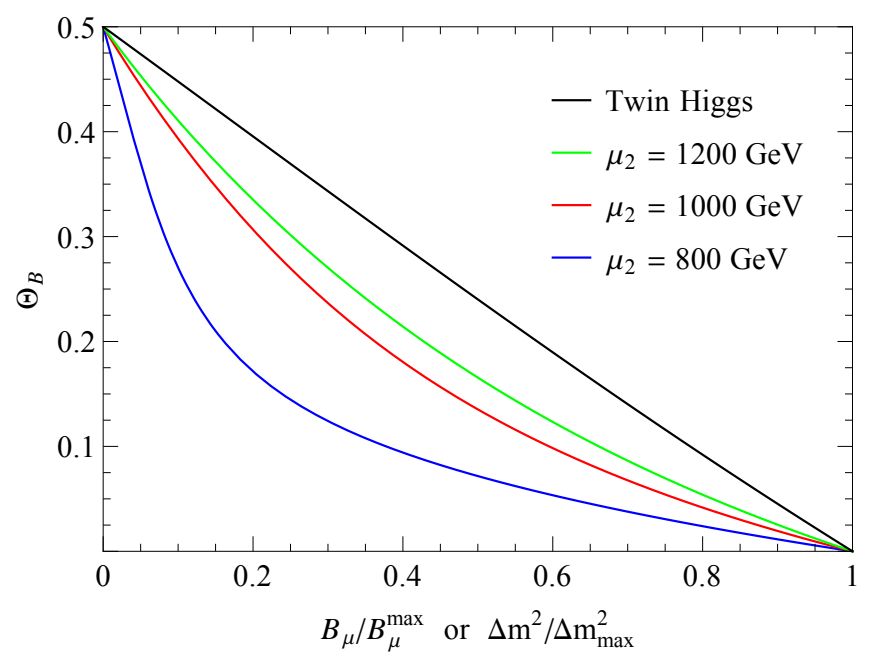

Figure 8.4: Example of $\Theta_{B}$ for the Twin Higgs and spontaneous $\mathbb{Z}_{2}$ breaking model with different values of $\mu_{2}$. The parameters for the Twin Higgs model are $\mu=750$ $\mathrm{GeV}, \alpha=-0.15$, and $\lambda=1$. The parameters for the spontaneous $\mathbb{Z}_{2}$ breaking model are $\mu_{1}=750 \mathrm{GeV}, \alpha_{1}=-0.15, \alpha_{2}=0.2$, and $\lambda_{1}=\lambda_{2}=1$.

\subsubsection{Radiative corrections}

In this section, we present the one loop leading radiative corrections for both the Twin Higgs and our model. Due to the similarities between both models, the radiative corrections are nearly identical for the two. The main differences result from the Twin Higgs only having a single SU(4) fundamental representation while our model contains two. These results are also similar to the radiative corrections given in [323], another Twin Higgs model with two SU(4) fundamental representations. The differences between their radiative corrections and ours follow from different forms of the quartic interactions.

To compute the radiative corrections, it is necessary to specify how the top couples to the different Higgses. In the Twin Higgs, this is encoded in the Lagrangian

$$
\mathcal{L}_{\text {top }}=-y_{t}\left(\bar{q}_{A} \tilde{H}_{A} t_{A}^{c}+\bar{q}_{B} \tilde{H}_{B} t_{B}^{c}\right)+\text { h.c. }
$$


where the $B$ sector quarks $q_{B}$ and $t_{B}^{c}$ do not carry Standard Model colour and the tilde notation stands for $\tilde{H}=i \sigma_{2} H^{*}$. The other Yukawa couplings can be safely ignored. The leading radiative corrections to the parameters of the Twin Higgs are then

$$
\begin{aligned}
\delta \mu^{2} & =\frac{1}{16 \pi^{2}}\left(6 y_{t}^{2}-\frac{9}{4} g^{2}-\frac{3}{4} g^{\prime 2}-10 \lambda-2 \alpha\right) \Lambda^{2}, \\
\delta \lambda & =\frac{1}{16 \pi^{2}}\left(6 y_{t}^{4}-\frac{9}{8} g^{4}-\frac{3}{4} g^{2} g^{\prime 2}-\frac{3}{8} g^{\prime 4}-32 \lambda^{2}-8 \lambda \alpha-2 \alpha^{2}\right) \log \frac{\Lambda}{f}, \\
\delta \alpha & =\frac{1}{16 \pi^{2}}\left(-12 y_{t}^{4}+\frac{9}{4} g^{4}+\frac{3}{2} g^{2} g^{\prime 2}+\frac{3}{4} g^{\prime 4}-24 \lambda \alpha\right) \log \frac{\Lambda}{f}, \\
\delta \Delta m^{2} & =\frac{1}{16 \pi^{2}}(-4 \lambda+4 \alpha) \Delta m^{2} \log \frac{\Lambda}{f},
\end{aligned}
$$

where $y_{t}$ is the top Yukawa coupling, $g$ and $g^{\prime}$ are the SM gauge couplings, and $\Lambda$ denotes the cutoff scale of the theory.

For our model, we must also specify how the top sector couples to the various Higgses. We choose the top to couple to $H_{1}$ only and to follow the structure of equation 8.25. The radiative corrections also depend on how the down-type quarks and the charged lepton couple to the Higgses, but the size of their Yukawa couplings makes these contributions irrelevant.

Another difference between our model and the Twin Higgs is that, in our case, radiative corrections also generate an additional operator of the form

$$
-\kappa\left(H_{1 A}^{\dagger} H_{1 A} H_{2 A}^{\dagger} H_{2 A}+H_{1 B}^{\dagger} H_{1 B} H_{2 B}^{\dagger} H_{2 B}\right)
$$

As mentioned above, the presence of such a term does not modify qualitatively the behaviour of the potential, as long as its coefficient is sufficiently small. We verified that this is the case for the operator of equation 8.30 with a coefficient of the size 
of its radiative correction. Even a considerably larger coefficient does not affect the behaviour much. Because of this, we limit ourselves to writing down its radiative correction and ignore it afterward. The leading radiative corrections then take the form

$$
\begin{aligned}
\delta \mu_{1}^{2} & =\frac{1}{16 \pi^{2}}\left(6 y_{t}^{2}-\frac{9}{4} g^{2}-\frac{3}{4} g^{\prime 2}-10 \lambda_{1}-2 \alpha_{1}\right) \Lambda^{2}, \\
\delta \lambda_{1} & =\frac{1}{16 \pi^{2}}\left(6 y_{t}^{4}-\frac{9}{8} g^{4}-\frac{3}{4} g^{2} g^{\prime 2}-\frac{3}{8} g^{\prime 4}-32 \lambda_{1}^{2}-8 \lambda_{1} \alpha_{1}-2 \alpha_{1}^{2}\right) \log \frac{\Lambda}{f_{1}} \\
\delta \alpha_{1} & =\frac{1}{16 \pi^{2}}\left(-12 y_{t}^{4}+\frac{9}{4} g^{4}+\frac{3}{2} g^{2} g^{\prime 2}+\frac{3}{4} g^{\prime 4}-24 \lambda_{1} \alpha_{1}\right) \log \frac{\Lambda}{f_{1}}, \\
\delta \mu_{2}^{2} & =\frac{1}{16 \pi^{2}}\left(-\frac{9}{4} g^{2}-\frac{3}{4} g^{\prime 2}-10 \lambda_{2}-2 \alpha_{2}\right) \Lambda^{2}, \\
\delta \lambda_{2} & =\frac{1}{16 \pi^{2}}\left(-\frac{9}{8} g^{4}-\frac{3}{4} g^{2} g^{\prime 2}-\frac{3}{8} g^{\prime 4}-32 \lambda_{2}^{2}-8 \lambda_{2} \alpha_{2}-2 \alpha_{2}^{2}\right) \log \frac{\Lambda}{f_{2}}, \\
\delta \alpha_{2} & =\frac{1}{16 \pi^{2}}\left(\frac{9}{4} g^{4}+\frac{3}{2} g^{2} g^{\prime 2}+\frac{3}{4} g^{\prime 4}-24 \lambda_{2} \alpha_{2}\right) \log \frac{\Lambda}{f_{2}}, \\
\delta B_{\mu} & =0 \\
\delta \kappa & =\frac{1}{16 \pi^{2}}\left(-\frac{9}{4} g^{4}-\frac{3}{2} g^{2} g^{\prime 2}-\frac{3}{4} g^{\prime 4}\right) \log \frac{\Lambda}{f_{1}} .
\end{aligned}
$$

For all radiative corrections presented above, we have neglected finite contributions.

\subsection{Numerical analysis of the fine-tuning}

In this section, we seek to compare more precisely the fine-tuning of our model to the original Twin Higgs. For both models, the fine-tuning comes from requesting a small $v / f$. In the case of the Twin Higgs, one has to tune the $\mathbb{Z}_{2}$ breaking sector against the $\mathrm{SU}(4)$ breaking sector. The tuning is evaluated in a similar way to [327] by defining

$$
\Delta_{\mathrm{TH}}=\left|\frac{\partial \log \left(v^{2} / f^{2}\right)}{\partial \log \Delta m^{2}}\right| .
$$


The tuning is then $\Delta_{\mathrm{TH}}^{-1}$. There are however a number of constraints that need to be satisfied. The vev $v$ and the mass of the lightest Higgs must be adjusted to their correct values, which we take to be $174.10 \mathrm{GeV}$ [328] and $125.09 \mathrm{GeV}$ [329] respectively. In addition, $f / v$ must be large enough to avoid experimental constraints. Setting this ratio to a given value imposes an additional constraint. Alternatively, one can set the fine-tuning to a given number and be interested in $f / v$, which can be used to estimate the cutoff.

There are four parameters in the Twin Higgs potential: $\mu^{2}, \lambda, \alpha$, and $\Delta m^{2}$. Matching $v$ and the mass of the Higgs with their respective values sets two parameters. Fixing $f / v$ or the tuning determines another one. We are therefore left with a single free parameter. For convenience sake, we take that parameter to be $\lambda$. We give two benchmarks. First, setting $\lambda=1$ and $f / v=3$ leads to a tuning of $27.7 \%$. Second, setting $\lambda=1$ and requesting a tuning of $20 \%$ leads to a $f / v$ of 3.42 .

A similar measure of fine-tuning can be defined in our model, but a few differences need to be taken into account. First, $B_{\mu}$ plays a similar role to $\Delta m^{2}$. As explained in section 8.2.2, one can obtain a very large ratio of vevs for a relatively small $B_{\mu} / B_{\mu}^{\max }$, given a very large mixing of $H_{1}$ and $H_{2}$. This however requires a fine-tuning of the parameters of the second Higgs $\left(\mu_{2}^{2}, \lambda_{2}\right.$, and $\left.\alpha_{2}\right)$ against those of the first. This tuning corresponds to $\Omega$ being close to 1 and needs to be taken into account. Second, there are simply more parameters in our case than in the original Twin Higgs. A measure that addresses all of these issues in a relatively fair manner is

$$
\Delta_{\text {Spontaneous }}=\operatorname{Max}\left\{\left|\frac{\partial \log \left(v^{2} / f_{1}^{2}\right)}{\partial \log B_{\mu}}\right|,\left|\frac{\partial \log \left(v^{2} / f_{1}^{2}\right)}{\partial \log \mu_{2}^{2}}\right|,\left|\frac{\partial \log \left(v^{2} / f_{1}^{2}\right)}{\partial \log \lambda_{2}}\right|,\left|\frac{\partial \log \left(v^{2} / f_{1}^{2}\right)}{\partial \log \alpha_{2}}\right|\right\} .
$$


The tuning is then $\Delta_{\text {Spontaneous. }}^{-1}{ }^{2}$ The number of parameters in the model is $7\left(\mu_{1}^{2}\right.$, $\mu_{2}^{2}, \lambda_{1}, \lambda_{2}, \alpha_{1}, \alpha_{2}$, and $\left.B_{\mu}\right)$. Three of them can be used to obtain the correct value of $v$ and the Higgs mass, as well as specifying $f_{1} / v$ or requesting a given tuning. A convenient choice is to use $\mu_{1}^{2}, \alpha_{1}$, and $B_{\mu}$ for this. The free parameters are then $\lambda_{1}$ and the parameters related to $H_{2}$ only. For convenience, we show all of the following plots for $\lambda_{1}=\lambda_{2}=1$. There are then two parameters left: $\mu_{2}^{2}$ and $\alpha_{2}$. As it makes the relation with the results of section 8.2.2 clearer, we present all contour plots in terms of $\mu_{2}^{2} / \mu_{1}^{2}$ and $-\alpha_{2} / \alpha_{1}$.

The left panel of figure 8.5 shows the tuning given a ratio $f_{1} / v$ of 3 . By inspecting equation 8.17 , one sees that the contour lines correspond roughly to lines of constant $\Omega$. The tuning also approaches a constant as $\Omega$ goes to 0 . This corresponds to the behaviour expected from the discussion of section 8.2.2. The grey area corresponds to the region of parameter space where the constraints do not accept any solution. It originates from the impossibility of creating a large enough hierarchy of vevs for $\Omega$ very close to 1 . The model is least fine-tuned when $\Omega$ is large enough for feedback to play an important role, while at the same time far away enough from 1 not to be considered fine-tuned. The ratio of the tuning to the corresponding Twin Higgs benchmark of $27.7 \%$ is shown in the right panel of figure 8.5. There is an optimal improvement of $58.1 \%$ and an improvement of $29.2 \%$ in the limit of $\Omega$ going to 0 . Conversely, the left panel of figure 8.6 shows $f_{1} / v$ for a fixed tuning of $20 \%$. The ratio of $f_{1} / v$ to the corresponding Twin Higgs benchmark of 3.42 can be seen in the right panel of figure 8.6. There is an optimal improvement of $22.5 \%$ and an improvement of $12.3 \%$ in the limit of $\Omega$ going to 0 .

\footnotetext{
${ }^{2}$ As in [327], we do not consider variations with respect to $\mu_{1}^{2}, \alpha_{1}$, and $\lambda_{1}$ and consider them to be fixed. Variations with respect to these parameters lead to slightly larger tuning, which is a consequence of $B_{\mu}^{\max }$ having cubic dependence on $f_{1}$. Our measure of tuning instead measures how close $B_{\mu}$ must be taken to $B_{\mu}^{\max }$ and how much the parameters of $H_{2}$ are adjusted with respect to those of $H_{1}$.
} 

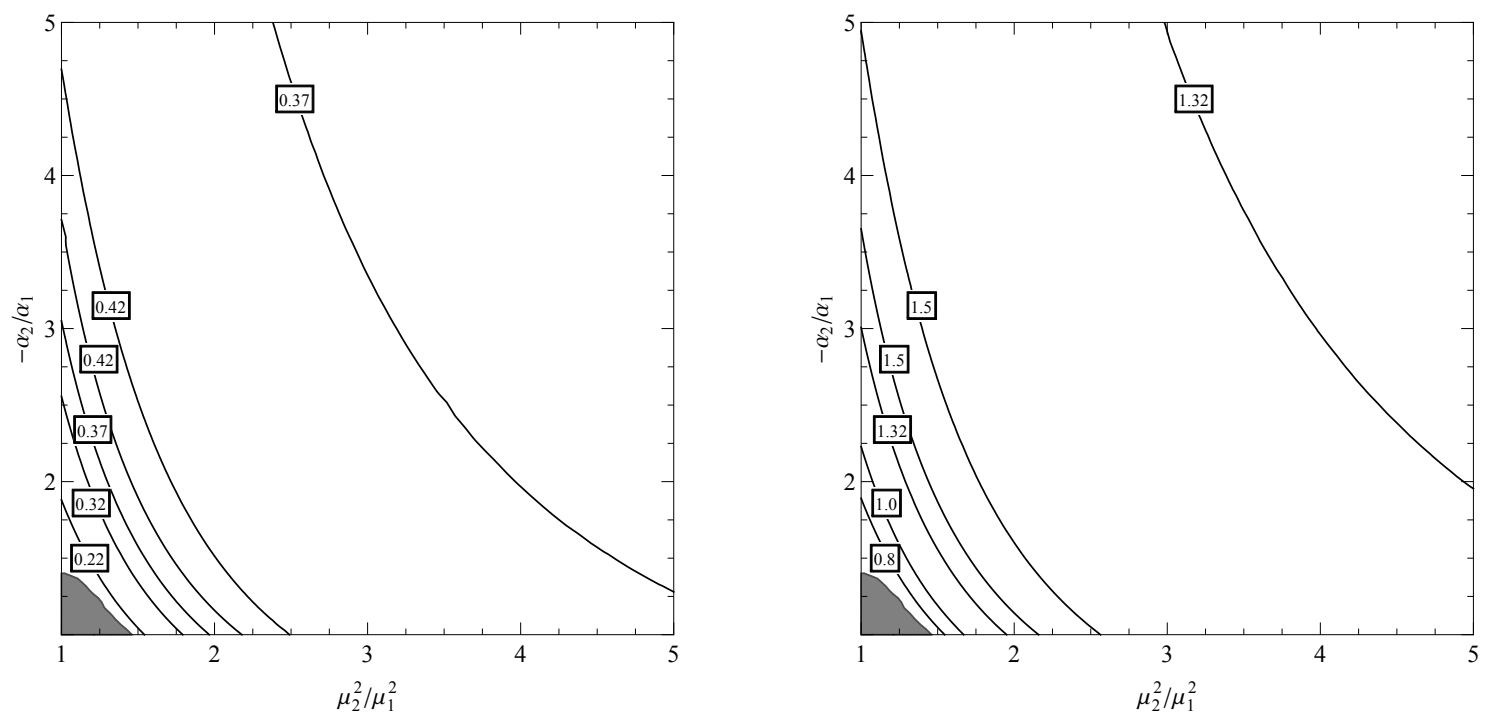

Figure 8.5: Tuning of the spontaneous $\mathbb{Z}_{2}$ breaking Twin Higgs for a fixed $f_{1} / v$ of 3. The left panel shows the tuning in percentage and the right one the ratio of the tuning to the Twin Higgs benchmark of $27.7 \%$. The grey area corresponds to the region where the constraints do not accept any solution.
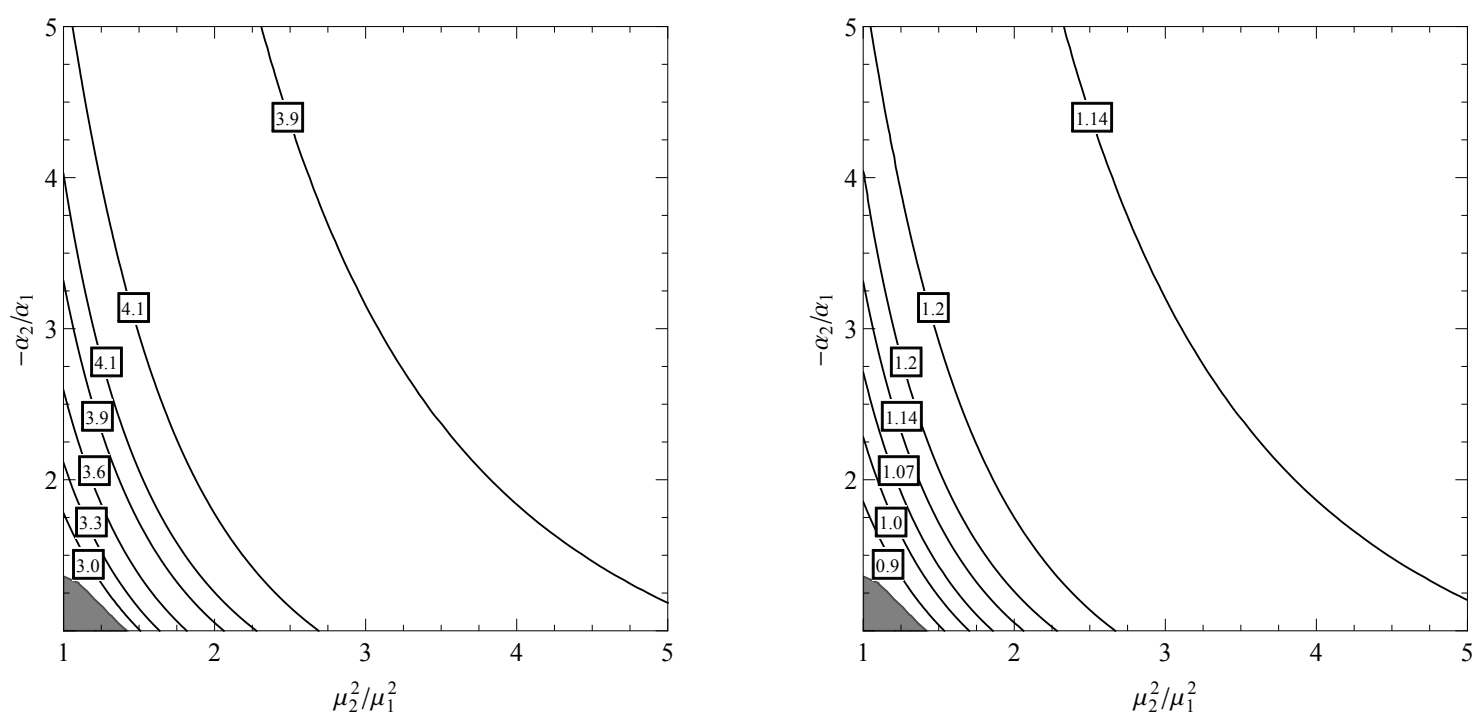

Figure 8.6: $f_{1} / v$ for the spontaneous $\mathbb{Z}_{2}$ breaking Twin Higgs for a fixed tuning of 20\%. The left panel shows $f_{1} / v$ and the right one the ratio of $f_{1} / v$ to the Twin Higgs benchmark of 3.42. The grey area corresponds to the region where the constraints do not accept any solution. 
Also of interest is the scale at which new physics is expected to become relevant, i.e. the cutoff. New physics is expected where the radiative corrections to the different parameters become large compared to their actual values. The bare parameters must then be tuned against their radiative corrections. The relevant parameters in both Twin Higgs and our model are those that receive quadratic corrections, i.e. the different $\mu^{2}$ 's. An estimate of the cutoff for a fixed tuning can be obtained by taking the value of $\Lambda$ for which the ratio of one of the $\mu^{2}$ 's and its radiative correction drops below said tuning. These corrections are only expected to give an order of magnitude estimate and are roughly given by $\left|\delta \mu^{2}\right| \sim 10 \lambda^{2} \Lambda^{2} /\left(16 \pi^{2}\right)$. Using the relations of section 8.2 , the results of $f / v$ can be used to estimate the cutoff. Requesting a tuning of $20 \%$ gives a cutoff of $7.5 \mathrm{TeV}$ for the Twin Higgs. In our model, the cutoff follows a similar pattern to figure 8.6 with an optimal value of $9.2 \mathrm{TeV}$ and a value of 8.4 $\mathrm{TeV}$ in the limit of $\Omega$ going to 0 .

\subsection{Conclusion}

In Twin Higgs models, the Higgs is a pseudo-Goldstone of a spontaneously broken approximate $\mathrm{SU}(4)$ global symmetry. It is kept light thanks to a $\mathbb{Z}_{2}$ symmetry that relates the Standard Model sector to a mirror sector. In order for the model to provide a hierarchy between the electroweak scale and the scale of new physics, an explicit $\mathbb{Z}_{2}$ breaking term is introduced and tuned against the small SU(4) breaking terms. In this chapter, we propose a Twin Higgs model where the $\mathbb{Z}_{2}$ symmetry is spontaneously broken. It consists of two Higgses that transform in the fundamental representation of a global SU(4). When they are decoupled, the vacuum of one of them preserves a $\mathbb{Z}_{2}$, while the other breaks it spontaneously. A $B_{\mu}$-like term that is bilinear in the two Higgses is then introduced. It acts as an effective tadpole and 
communicates the $\mathbb{Z}_{2}$ breaking from one sector to the other, resulting in a hierarchy of vevs. This effective tadpole and the feedback between the two Higgses lead to a milder tuning than in the original Twin Higgs.

The phenomenology of the model is quite similar to that of the Twin Higgs. It contains a mirror sector that is not charged under the Standard Model. The two sectors communicate weakly through the Higgs but, as mentioned above, the mixing of the Standard Model Higgs with the $B$-sector is smaller in our model than in the Twin Higgs. On the other hand, this model is a two Higgs doublet model which could lead to additional signatures.

The next logical question concerns a possible UV completion. The obvious guess would be a supersymmetric version of the model. However, SUSY generally leads to a more complicated quartic structure than equations 8.11 and 8.12 . This prevents the model from being translated directly to SUSY. In addition, getting the correct sign of the $\alpha_{i}$ 's generally proves to be problematic. The combination of the D-terms and the largest loop corrections provides a negative contribution to the $\alpha_{i}$ of both the up and down Higgses [327]. The terms leading to spontaneous $\mathbb{Z}_{2}$ breaking must therefore originate from the superpotential. One possibility would be to introduce a superpotential term of the form

$$
\lambda H_{d A} U H_{d B},
$$

where $U$ is a fundamental representation of both $\mathrm{SU}(2)_{A}$ and $\mathrm{SU}(2)_{B}$ and has the appropriate weak hypercharges. Assuming a very large soft mass for $U$ and integrating it out would lead to a positive contribution to $\alpha_{d}$ and can lead to the correct $\mathbb{Z}_{2}$ breaking structure.

One other possibility would be to have both $H_{u}$ and $H_{d}$ preserve $\mathbb{Z}_{2}$, but include 
a NMSSM-like scalar sector that spontaneously breaks $\mathbb{Z}_{2}$. For example, consider the superpotential

$$
W=\lambda^{\prime} S^{\prime}\left(S_{A}^{2}+S_{B}^{2}\right)+\lambda^{\prime \prime} S^{\prime \prime} S_{A} S_{B}
$$

and assume that both $S^{\prime}$ and $S^{\prime \prime}$ have large soft masses and that $S=\left(S_{A}, S_{B}\right)$ has a negative soft mass squared. The first term preserves a global $\mathrm{O}(2)$ symmetry that the second term breaks. Both terms preserve the $\mathbb{Z}_{2}$ symmetry. However, this symmetry will be broken spontaneously. If $S_{A}$ couples to the $A$-type Higgses and $S_{B}$ to the $B$-type Higgses, the symmetry breaking is transmitted to the Higgs sector as well. Of course, the viability of these models would require studies of their own. 


\section{Chapter 9}

\section{Summary}

Predominately motivated by the apparent unnaturalness of the electroweak scale in the SM, this thesis investigated models of weak scale supersymmetry and a variation of the Twin Higgs model. As supersymmetry also has other benefits such as providing dark matter candidates and potential gauge coupling unification, an unnatural model of supersymmetry with these properties called Mini-Split was also studied. New experimental data, primarily collected during run 1 and run 2 of the LHC, was used to either constrain the models under consideration or to motivate the exploration of the possible beyond the SM extension. Specifically, this thesis contains five projects whose main ideas and results are summarized below.

Chapter 4 considered the Mini-Split model of supersymmetry. In these models, the masses of the gauginos are a few orders of magnitude lighter than the masses of the scalars. Further, the masses of the Higgsinos can be at a scale similar to the gauginos or, as was examined in this thesis, at a scale similar to the scalars. The heavy scalars and Higgsinos then deflect the gaugino masses from their standard anomaly and gauge mediation trajectories, with the resulting pole masses given in equation 4.10 for

anomaly mediation and equation 4.13 for gauge mediation. Assuming a slightly lighter 
third generation of squarks, the phenomenology of the gauginos (based primarily on gluino pair production) was analyzed and constraints on these Mini-Split models were derived. Future prospects at LHC 14 and a $100 \mathrm{TeV}$ proton proton collider were also determined. The results are given in section 4.3.

Chapter 5 studied the pair production of Higgsino neutralinos and charginos at the LHC assuming they promptly decay due to a single non-zero $R$-parity violating term in the superpotential of the form $\lambda_{3 j k}^{\prime \prime} U_{3}^{c} D_{j}^{c} D_{k}^{c}$. First shown in this chapter is that if the mass splitting between the two Higgsino neutralino states is greater than their widths, then pair produced neutralinos decay equally often into final states with same sign tops as they do into final states with opposite sign tops. Same sign tops can lead to same sign leptons, and by recasting a CMS search looking for this distinctive phenomenological signature together with multiple b-jets, novel constraints on the parameter space were derived. More precisely, we performed a scan over the parameter space and plotted the excluded points projected onto different axes as shown in figure 5.4. Benchmark exclusion curves are also presented in figure 5.5.

Chapter 6 introduced an $R$-symmetric model of supersymmetry where the $R$ symmetry is identified with baryon number. This allows the $R$-parity violating operator $\lambda^{\prime \prime} U^{c} D^{c} D^{c}$ in the superpotential without breaking baryon number. However, the $R$-symmetry cannot remain an exact symmetry of the model as it will be broken by at least anomaly mediation. This reintroduces bounds coming from baryon number violating processes which are presented in section 6.2.1.1. Bounds coming from flavour physics remain the same as in the RPVMSSM and are presented in section 6.2.1.2. We also considered the LHC phenomenology of both stops and the first two generations of squarks assuming they decay due to a single non-zero coupling of the form $\lambda_{3 j k}^{\prime \prime}$ to final states featuring only SM particles. The production of same sign tops from, for 
example, a decaying stop is forbidden if the $R$-symmetry is unbroken, which simply follows from conservation of baryon number. However, the same sign top signature is regenerated once the breaking of the $R$-symmetry is taken into account and provided that the resulting mass splitting between the now pseudo-Dirac neutralino states is greater than their widths. Thus, we considered two phenomenological cases: one in which the $R$-symmetry is unbroken and the other in which it is sufficiently broken so that same sign tops are produced as often as opposite sign tops. Parameter regions excluded due to stop phenomenology are shown in section 6.3.1 and parameter regions excluded due to the phenomenology of the first two generations of squarks are shown in section 6.3.2.

Chapter 7 investigated the experimentally observed $B$ meson anomalies related to the quark level decays $b \rightarrow s \ell$. This thesis attempted to explain these anomalies by using the $R$-parity violating superpotential operator $\lambda^{\prime} L Q D^{c}$. To do so, contributions to the four-fermi effective operator $\left(\bar{s} \gamma_{\alpha} P_{L} b\right)\left(\bar{\mu} \gamma^{\alpha} P_{L} \mu\right)$ were calculated from one loop diagrams. These one loop diagrams included box diagrams with an internal $W$ boson, an internal wino, and four $\lambda^{\prime}$ vertices, as well as photonic and $Z$ penguin diagrams. The results of these calculations are presented in section 7.2. This project focused on the parameter space in which diagrams involving winos gave large contributions. As presented in section 7.3, many different potentially constraining processes were considered including $\tau \rightarrow \mu \mu \mu, B_{s}-\bar{B}_{s}$ mixing, $B \rightarrow K^{(*)} \nu \bar{\nu}, Z$ decays to charged leptons, and direct LHC searches. Our results are presented in figure 7.6, which shows that this setup can potentially explain the anomalies, but it requires large values of $\lambda^{\prime}$, and this leads to relatively low Landau poles.

Chapter 8 presented a variation of the Twin Higgs model. Starting from the original model, we first expanded the Higgs sector by including a second fundamental 
representation of the global SU(4) symmetry. The parameters were chosen so that the vev of one of the fundamental SU(4) Higgs fields preserved the $\mathbb{Z}_{2}$ symmetry while the vev of the other Higgs field broke it spontaneously. This spontaneous breaking of the $\mathbb{Z}_{2}$ symmetry is the novel feature in our model and contrasts with the explicit breaking of the $\mathbb{Z}_{2}$ symmetry in the original Twin Higgs model. Further, a term was included in the scalar potential to communicate the breaking of the $\mathbb{Z}_{2}$ symmetry from the broken to the unbroken sector. We showed that this has many benefits, including a reduction in the amount of tuning compared to that in the original Twin Higgs as demonstrated in figure 8.5 (see also figure 8.6). 


\section{Appendix A}

\section{One loop beta functions used in the investigation of $b \rightarrow s \ell \ell$ anomalies}

As described in greater detail in section 7.3.6, the parameter space potentially capable of explaining the anomalies in the $b \rightarrow s \ell \ell$ transitions generically results in Landau poles below the Planck scale. As also described in this section, three steps were taken to determine the energy scale of these Landau poles. The first step was to evolve the three gauge couplings and the top, bottom, and tau Yukawa couplings up to the left-handed up squarks mass scale using the Standard Model beta functions. The second step was to evolve these parameters and the four $\lambda^{\prime}$ couplings up to the righthanded sbottom and sneutrino mass scale. This second evolution assumes that all the sfermions of the superfields $U^{c}, D^{c}, L$, and $E^{c}$ are at this heavy mass scale and thus their affects on the renormalization group equations decouple. The final step was to evolve the ten parameters from the right-handed sbottom and sneutrino mass scale upwards taking into account the affects of all the sfermions and to determine the energy scale of Landau poles accordingly.

In this appendix, we present the one loop beta functions used to perform the 
evolution of the ten parameters from the left-handed up squarks mass scale to the right-handed sbottom and sneutrino mass scale and from the right-handed sbottom and sneutrino mass scale upwards. First, note that the renormalization group equation for a generic coupling $\lambda$ is given by the equation

$$
\frac{d \lambda}{d t}=\beta_{\lambda}
$$

where $\beta_{\lambda}$ is $\lambda$ 's beta function and $t=\log \left(Q / Q_{0}\right)$. Next, the ten couplings are

$$
g_{1}=\sqrt{\frac{5}{3}} g^{\prime}, \quad g_{2}=g, \quad g_{3}=g_{s}, \quad y_{t}, \quad y_{b}, \quad y_{\tau}, \quad \lambda_{223}^{\prime}, \quad \lambda_{233}^{\prime}, \quad \lambda_{323}^{\prime}, \quad \lambda_{333}^{\prime}
$$

where we use $\mathrm{SU}(5)$ normalization for the hypercharge coupling constant and the $y_{i}$ are the Yukawa couplings. Also, we make the simplifying approximation that the CKM matrix is equal to the identity matrix so that $\tilde{\lambda}_{i j k}^{\prime}=\lambda_{i j k}^{\prime}$ (cf. equation 7.10). The two sets of beta functions presented below were computed by examining every relevant one loop diagram affecting the renormalization of the parameters under consideration.

The one loop beta functions to evolve these parameters from the left-handed up squarks mass scale to the right-handed sbottom and sneutrino mass scale are

$$
\begin{aligned}
& (4 \pi)^{2} \beta_{g_{1}}=\frac{47}{10} g_{1}^{3} \\
& (4 \pi)^{2} \beta_{g_{2}}=\frac{1}{2} g_{2}^{3} \\
& (4 \pi)^{2} \beta_{g_{3}}=-4 g_{3}^{3} \\
& (4 \pi)^{2} \beta_{y_{t}}=y_{t}\left(-\frac{1}{3} g_{1}^{2}-3 g_{2}^{2}-\frac{20}{3} g_{3}^{2}+\frac{11}{2} y_{t}^{2}+\frac{1}{2} y_{b}^{2}\right)
\end{aligned}
$$




$$
\begin{aligned}
(4 \pi)^{2} \beta_{y_{b}}= & y_{b}\left(-\frac{2}{15} g_{1}^{2}-3 g_{2}^{2}-\frac{20}{3} g_{3}^{2}+\frac{1}{2} y_{t}^{2}+\frac{11}{2} y_{b}^{2}+y_{\tau}^{2}\right. \\
& \left.+\left|\lambda_{223}^{\prime}\right|^{2}+\left|\lambda_{233}^{\prime}\right|^{2}+\left|\lambda_{323}^{\prime}\right|^{2}+\left|\lambda_{333}^{\prime}\right|^{2}\right) \\
(4 \pi)^{2} \beta_{y_{\tau}}= & y_{\tau}\left(-\frac{39}{20} g_{1}^{2}-\frac{3}{4} g_{2}^{2}+3 y_{b}^{2}+\frac{5}{2} y_{\tau}^{2}+\frac{3}{2}\left|\lambda_{323}^{\prime}\right|^{2}+\frac{3}{2}\left|\lambda_{333}^{\prime}\right|^{2}\right) \\
(4 \pi)^{2} \beta_{\lambda_{223}^{\prime}}= & \lambda_{223}^{\prime}\left(-\frac{37}{60} g_{1}^{2}-\frac{3}{4} g_{2}^{2}-\frac{4}{3} g_{3}^{2}+2 y_{b}^{2}\right)+\frac{5}{2} \lambda_{233}^{\prime} \lambda_{323}^{\prime} \lambda_{333}^{*} \\
+ & \frac{7}{2} \lambda_{223}^{\prime}\left|\lambda_{223}^{\prime}\right|^{2}+\frac{7}{2} \lambda_{223}^{\prime}\left|\lambda_{233}^{\prime}\right|^{2}+\frac{7}{2} \lambda_{223}^{\prime}\left|\lambda_{323}^{\prime}\right|^{2}+\lambda_{223}^{\prime}\left|\lambda_{333}^{\prime}\right|^{2} \\
(4 \pi)^{2} \beta_{\lambda_{233}^{\prime}}= & \lambda_{233}^{\prime}\left(-\frac{37}{60} g_{1}^{2}-\frac{3}{4} g_{2}^{2}-\frac{4}{3} g_{3}^{2}+y_{t}^{2}+\frac{9}{2} y_{b}^{2}\right)+\frac{5}{2} \lambda_{223}^{\prime} \lambda_{333}^{\prime} \lambda_{323}^{\prime *} \\
+ & \frac{7}{2} \lambda_{233}^{\prime}\left|\lambda_{223}^{\prime}\right|^{2}+\frac{7}{2} \lambda_{233}^{\prime}\left|\lambda_{233}^{\prime}\right|^{2}+\frac{7}{2} \lambda_{233}^{\prime}\left|\lambda_{333}^{\prime}\right|^{2}+\lambda_{233}^{\prime}\left|\lambda_{323}^{\prime}\right|^{2} \\
= & \lambda_{323}^{\prime}\left(-\frac{37}{60} g_{1}^{2}-\frac{3}{4} g_{2}^{2}-\frac{4}{3} g_{3}^{2}+2 y_{b}^{2}+\frac{1}{2} y_{\tau}^{2}\right)+\frac{5}{2} \lambda_{223}^{\prime} \lambda_{333}^{\prime} \lambda_{233}^{\prime *} \\
(4 \pi)^{2} \beta_{\lambda_{323}^{\prime}} & \frac{7}{2} \lambda_{323}^{\prime}\left|\lambda_{223}^{\prime}\right|^{2}+\frac{7}{2} \lambda_{323}^{\prime}\left|\lambda_{323}^{\prime}\right|^{2}+\frac{7}{2} \lambda_{323}^{\prime}\left|\lambda_{333}^{\prime}\right|^{2}+\lambda_{323}^{\prime}\left|\lambda_{233}^{\prime}\right|^{2} \\
= & \lambda_{333}^{\prime}\left(-\frac{37}{60} g_{1}^{2}-\frac{3}{4} g_{2}^{2}-\frac{4}{3} g_{3}^{2}+y_{t}^{2}+\frac{9}{2} y_{b}^{2}+\frac{1}{2} y_{\tau}^{2}\right)+\frac{5}{2} \lambda_{233}^{\prime} \lambda_{323}^{\prime} \lambda_{223}^{\prime *} \\
+ & \frac{7}{2} \lambda_{333}^{\prime}\left|\lambda_{233}^{\prime}\right|^{2}+\frac{7}{2} \lambda_{333}^{\prime}\left|\lambda_{323}^{\prime}\right|^{2}+\frac{7}{2} \lambda_{333}^{\prime}\left|\lambda_{333}^{\prime}\right|^{2}+\lambda_{333}^{\prime}\left|\lambda_{223}^{\prime}\right|^{2} \\
(4 \pi)^{2} \beta_{\lambda_{333}^{\prime}} & (A .1
\end{aligned}
$$

Finally, the one loop beta functions to evolve these parameters from the right-handed sbottom and sneutrino mass scale upwards are

$$
\begin{aligned}
&(4 \pi)^{2} \beta_{g_{1}}= \frac{33}{5} g_{1}^{3} \\
&(4 \pi)^{2} \beta_{g_{2}}= g_{2}^{3} \\
&(4 \pi)^{2} \beta_{g_{3}}=-3 g_{3}^{3} \\
&(4 \pi)^{2} \beta_{y_{t}}=y_{t}\left(-\frac{13}{15} g_{1}^{2}-3 g_{2}^{2}-\frac{16}{3} g_{3}^{2}+6 y_{t}^{2}+y_{b}^{2}+\left|\lambda_{233}^{\prime}\right|^{2}+\left|\lambda_{333}^{\prime}\right|^{2}\right) \\
&(4 \pi)^{2} \beta_{y_{b}}=y_{b}\left(-\frac{7}{15} g_{1}^{2}-3 g_{2}^{2}-\frac{16}{3} g_{3}^{2}+y_{t}^{2}+6 y_{b}^{2}+y_{\tau}^{2}\right. \\
&\left.\quad+2\left|\lambda_{223}^{\prime}\right|^{2}+6\left|\lambda_{233}^{\prime}\right|^{2}+2\left|\lambda_{323}^{\prime}\right|^{2}+6\left|\lambda_{333}^{\prime}\right|^{2}\right)
\end{aligned}
$$




$$
\begin{aligned}
(4 \pi)^{2} \beta_{y_{\tau}} & =y_{\tau}\left(-\frac{9}{5} g_{1}^{2}-3 g_{2}^{2}+3 y_{b}^{2}+4 y_{\tau}^{2}+3\left|\lambda_{323}^{\prime}\right|^{2}+3\left|\lambda_{333}^{\prime}\right|^{2}\right) \\
(4 \pi)^{2} \beta_{\lambda_{223}^{\prime}} & =\lambda_{223}^{\prime}\left(-\frac{7}{15} g_{1}^{2}-3 g_{2}^{2}-\frac{16}{3} g_{3}^{2}+2 y_{b}^{2}\right)+4 \lambda_{233}^{\prime} \lambda_{323}^{\prime} \lambda_{333}^{\prime *} \\
& +6 \lambda_{223}^{\prime}\left|\lambda_{223}^{\prime}\right|^{2}+6 \lambda_{223}^{\prime}\left|\lambda_{233}^{\prime}\right|^{2}+6 \lambda_{223}^{\prime}\left|\lambda_{323}^{\prime}\right|^{2}+2 \lambda_{223}^{\prime}\left|\lambda_{333}^{\prime}\right|^{2} \\
(4 \pi)^{2} \beta_{\lambda_{233}^{\prime}} & =\lambda_{233}^{\prime}\left(-\frac{7}{15} g_{1}^{2}-3 g_{2}^{2}-\frac{16}{3} g_{3}^{2}+y_{t}^{2}+6 y_{b}^{2}\right)+4 \lambda_{223}^{\prime} \lambda_{333}^{\prime} \lambda_{323}^{\prime *} \\
& +6 \lambda_{233}^{\prime}\left|\lambda_{223}^{\prime}\right|^{2}+6 \lambda_{233}^{\prime}\left|\lambda_{233}^{\prime}\right|^{2}+6 \lambda_{233}^{\prime}\left|\lambda_{333}^{\prime}\right|^{2}+2 \lambda_{233}^{\prime}\left|\lambda_{323}^{\prime}\right|^{2} \\
(4 \pi)^{2} \beta_{\lambda_{323}^{\prime}} & =\lambda_{323}^{\prime}\left(-\frac{7}{15} g_{1}^{2}-3 g_{2}^{2}-\frac{16}{3} g_{3}^{2}+2 y_{b}^{2}+y_{\tau}^{2}\right)+4 \lambda_{223}^{\prime} \lambda_{333}^{\prime} \lambda_{233}^{\prime *} \\
& +6 \lambda_{323}^{\prime}\left|\lambda_{223}^{\prime}\right|^{2}+6 \lambda_{323}^{\prime}\left|\lambda_{323}^{\prime}\right|^{2}+6 \lambda_{323}^{\prime}\left|\lambda_{333}^{\prime}\right|^{2}+2 \lambda_{323}^{\prime}\left|\lambda_{233}^{\prime}\right|^{2} \\
& \lambda_{333}^{\prime}\left(-\frac{7}{15} g_{1}^{2}-3 g_{2}^{2}-\frac{16}{3} g_{3}^{2}+y_{t}^{2}+6 y_{b}^{2}+y_{\tau}^{2}\right)+4 \lambda_{233}^{\prime} \lambda_{323}^{\prime} \lambda_{223}^{\prime *} \\
& +6 \lambda_{333}^{\prime}\left|\lambda_{233}^{\prime}\right|^{2}+6 \lambda_{333}^{\prime}\left|\lambda_{323}^{\prime}\right|^{2}+6 \lambda_{333}^{\prime}\left|\lambda_{333}^{\prime}\right|^{2}+2 \lambda_{333}^{\prime}\left|\lambda_{223}^{\prime}\right|^{2} .
\end{aligned}
$$

The beta functions in these last ten equations can also be determined by using the results in [291]. 


\section{References}

[1] H. Beauchesne, K. Earl, and T. Grégoire, LHC constraints on Mini-Split anomaly and gauge mediation and prospects for LHC 14 and a future $100 \mathrm{TeV}$ pp collider, JHEP 08 (2015) 117, [arXiv: 1503.03099].

[2] H. Beauchesne, K. Earl, and T. Gregoire, LHC phenomenology and baryogenesis in supersymmetric models with a $U(1)_{R}$ baryon number, JHEP 06 (2017) 122, [arXiv: 1703.03866].

[3] K. Earl and T. Grégoire, Contributions to $b \rightarrow$ sll Anomalies from R-Parity Violating Interactions, JHEP 08 (2018) 201, [arXiv: 1806.01343].

[4] H. Beauchesne, K. Earl, and T. Grégoire, The spontaneous $\mathbb{Z}_{2}$ breaking Twin Higgs, JHEP 01 (2016) 130, [arXiv:1510.06069].

[5] M. E. Peskin and D. V. Schroeder, An Introduction to quantum field theory. Addison-Wesley, Reading, USA, 1995.

[6] M. D. Schwartz, Quantum Field Theory and the Standard Model. Cambridge University Press, 2014.

[7] M. Srednicki, Quantum field theory. Cambridge University Press, 2007.

[8] S. Weinberg, The Quantum theory of fields. Vol. 1: Foundations. Cambridge University Press, 2005.

[9] H. Georgi, Lie algebras in particle physics, Front. Phys. 54 (1999) 1-320.

[10] E. P. Wigner, On Unitary Representations of the Inhomogeneous Lorentz Group, Annals Math. 40 (1939) 149-204. [Reprint: Nucl. Phys. Proc. Suppl.6,9(1989)].

[11] R. Rajaraman, Solitons And Instantons. An Introduction To Solitons And Instantons In Quantum Field Theory, Amsterdam, Netherlands: North-holland (1982) 409p (1982). 
[12] R. Contino, The Higgs as a Composite Nambu-Goldstone Boson, in Physics of the large and the small, TASI 09, proceedings of the Theoretical Advanced Study Institute in Elementary Particle Physics, Boulder, Colorado, USA, 1-26 June 2009, pp. 235-306, 2011. arXiv:1005.4269.

[13] M. C. Gonzalez-Garcia and M. Maltoni, Phenomenology with Massive Neutrinos, Phys. Rept. 460 (2008) 1-129, [arXiv:0704.1800].

[14] S. Weinberg, Baryon and Lepton Nonconserving Processes, Phys. Rev. Lett. 43 (1979) 1566-1570.

[15] P. Labelle, Supersymmetry demystified. McGraw-Hill, New York, NY, 2010.

[16] S. P. Martin, A Supersymmetry primer, hep-ph/9709356. [Adv. Ser. Direct. High Energy Phys.18,1(1998)].

[17] S. R. Coleman and J. Mandula, All Possible Symmetries of the S Matrix, Phys. Rev. 159 (1967) 1251-1256.

[18] R. Haag, J. T. Lopuszanski, and M. Sohnius, All Possible Generators of Supersymmetries of the s Matrix, Nucl. Phys. B88 (1975) 257. [,257(1974)].

[19] R. Barbier et al., R-parity violating supersymmetry, Phys. Rept. 420 (2005) 1-202, [hep-ph/0406039].

[20] M. Dine and W. Fischler, A Phenomenological Model of Particle Physics Based on Supersymmetry, Phys.Lett. B110 (1982) 227.

[21] C. R. Nappi and B. A. Ovrut, Supersymmetric Extension of the SU(3) $x$ SU(2) $x$ U(1) Model, Phys.Lett. B113 (1982) 175.

[22] L. Alvarez-Gaume, M. Claudson, and M. B. Wise, Low-Energy Supersymmetry, Nucl.Phys. B207 (1982) 96.

[23] M. Dine and A. E. Nelson, Dynamical supersymmetry breaking at low-energies, Phys.Rev. D48 (1993) 1277-1287, [hep-ph/9303230].

[24] M. Dine, A. E. Nelson, and Y. Shirman, Low-energy dynamical supersymmetry breaking simplified, Phys.Rev. D51 (1995) 1362-1370, [hep-ph/9408384].

[25] M. Dine, A. E. Nelson, Y. Nir, and Y. Shirman, New tools for low-energy dynamical supersymmetry breaking, Phys.Rev. D53 (1996) 2658-2669, [hep-ph/9507378].

[26] L. Randall and R. Sundrum, Out of this world supersymmetry breaking, Nucl.Phys. B557 (1999) 79-118, [hep-th/9810155]. 
[27] G. F. Giudice, M. A. Luty, H. Murayama, and R. Rattazzi, Gaugino mass without singlets, JHEP 9812 (1998) 027, [hep-ph/9810442].

[28] A. H. Chamseddine, R. L. Arnowitt, and P. Nath, Locally Supersymmetric Grand Unification, Phys. Rev. Lett. 49 (1982) 970.

[29] J. D. Wells, Implications of supersymmetry breaking with a little hierarchy between gauginos and scalars, hep-ph/0306127.

[30] N. Arkani-Hamed and S. Dimopoulos, Supersymmetric unification without low energy supersymmetry and signatures for fine-tuning at the LHC, JHEP 0506 (2005) 073, [hep-th/0405159].

[31] G. Giudice and A. Romanino, Split supersymmetry, Nucl.Phys. B699 (2004) 65-89, [hep-ph/0406088].

[32] A. Arvanitaki, N. Craig, S. Dimopoulos, and G. Villadoro, Mini-Split, JHEP 1302 (2013) 126, [arXiv: 1210.0555].

[33] CMS Collaboration, S. Chatrchyan et al., Measurement of the properties of a Higgs boson in the four-lepton final state, Phys.Rev. D89 (2014) 092007, [arXiv: 1312.5353].

[34] ATLAS Collaboration, G. Aad et al., Measurement of the Higgs boson mass from the $H \rightarrow \gamma \gamma$ and $H \rightarrow Z Z^{*} \rightarrow 4 \ell$ channels with the ATLAS detector using $25 \mathrm{fb}^{-1}$ of pp collision data, Phys.Rev. D90 (2014) 052004, [arXiv: 1406.3827].

[35] R. Rattazzi, A. Strumia, and J. D. Wells, Phenomenology of deflected anomaly mediation, Nucl.Phys. B576 (2000) 3-28, [hep-ph/9912390].

[36] E. Bagnaschi, G. F. Giudice, P. Slavich, and A. Strumia, Higgs Mass and Unnatural Supersymmetry, JHEP 1409 (2014) 092, [arXiv:1407.4081].

[37] S. Jung and J. D. Wells, Gaugino physics of split supersymmetry spectrum at the LHC and future proton colliders, Phys.Rev. D89 (2014) 075004, [arXiv:1312.1802].

[38] A. Cesarini, F. Fucito, and A. Lionetto, Deflected Anomaly Mediation and Neutralino Dark Matter, Phys.Rev. D75 (2007) 025026, [hep-ph/0611098].

[39] N. Yokozaki, Electroweak Symmetry Breaking and Singlino Dark Matter with Deflected Anomaly Mediation, JHEP 0905 (2009) 095, [arXiv:0903.2632].

[40] N. Setzer and S. Spinner, When Anomaly Mediation is UV Sensitive, JHEP 1106 (2011) 137, [arXiv: 1008.3774]. 
[41] N. Okada, Positively deflected anomaly mediation, Phys.Rev. D65 (2002) 115009, [hep-ph/0202219].

[42] N. Okada and H. M. Tran, Positively deflected anomaly mediation in the light of the Higgs boson discovery, Phys.Rev. D87 (2013) 035024, [arXiv:1212.1866].

[43] J. de Blas and A. Delgado, Singlet deflected anomaly/gauge mediation, Phys.Lett. B708 (2012) 300-306, [arXiv:1108.2511].

[44] M. Ibe, T. Moroi, and T. Yanagida, Possible Signals of Wino LSP at the Large Hadron Collider, Phys.Lett. B644 (2007) 355-360, [hep-ph/0610277].

[45] M. Ibe and T. T. Yanagida, The Lightest Higgs Boson Mass in Pure Gravity Mediation Model, Phys.Lett. B709 (2012) 374-380, [arXiv:1112.2462].

[46] M. Ibe, S. Matsumoto, and T. T. Yanagida, Pure Gravity Mediation with $m_{3 / 2}$ = 10-100TeV, Phys.Rev. D85 (2012) 095011, [arXiv:1202.2253].

[47] B. Bhattacherjee, B. Feldstein, M. Ibe, S. Matsumoto, and T. T. Yanagida, Pure gravity mediation of supersymmetry breaking at the Large Hadron Collider, Phys.Rev. D87 (2013), no. 1 015028, [arXiv:1207.5453].

[48] Y. Kahn, M. McCullough, and J. Thaler, Auxiliary Gauge Mediation: A New Route to Mini-Split Supersymmetry, JHEP 1311 (2013) 161, [arXiv:1308.3490].

[49] ATLAS Collaboration, Search for chargino and neutralino production in final states with one lepton, two b-jets consistent with a Higgs boson, and missing transverse momentum with the ATLAS detector in $20.3 \mathrm{fb}^{-1}$ of $\sqrt{\mathrm{s}}=$ 8 TeV pp collisions, ATLAS-CONF-2013-093, ATLAS-COM-CONF-2013-102.

[50] ATLAS Collaboration, G. Aad et al., Search for direct production of charginos, neutralinos and sleptons in final states with two leptons and missing transverse momentum in pp collisions at $\sqrt{s}=8$ TeV with the ATLAS detector, JHEP 1405 (2014) 071, [arXiv:1403.5294].

[51] ATLAS Collaboration, G. Aad et al., Search for direct production of charginos and neutralinos in events with three leptons and missing transverse momentum in $\sqrt{s}=8 T e V p p$ collisions with the ATLAS detector, JHEP 1404 (2014) 169, [arXiv: 1402.7029].

[52] ATLAS Collaboration, G. Aad et al., Search for supersymmetry at $\sqrt{s}=8$ $T e V$ in final states with jets and two same-sign leptons or three leptons with the ATLAS detector, JHEP 1406 (2014) 035, [arXiv:1404.2500]. 
[53] ATLAS Collaboration, G. Aad et al., Search for strong production of supersymmetric particles in final states with missing transverse momentum and at least three $b$-jets at $\sqrt{s}=8 \mathrm{TeV}$ proton-proton collisions with the ATLAS detector, JHEP 1410 (2014) 24, [arXiv:1407.0600].

[54] CMS Collaboration, V. Khachatryan et al., Searches for electroweak neutralino and chargino production in channels with Higgs, $Z$, and $W$ bosons in pp collisions at 8 TeV, Phys.Rev. D90 (2014) 092007, [arXiv:1409.3168].

[55] CMS Collaboration, S. Chatrchyan et al., Search for new physics in the multijet and missing transverse momentum final state in proton-proton collisions at $\sqrt{s}=8 \mathrm{TeV}$, JHEP 1406 (2014) 055, [arXiv:1402.4770].

[56] CMS Collaboration, Search for supersymmetry in pp collisions at sqrt(s) = $8 \mathrm{Tev}$ in events with two opposite sign leptons, large number of jets, b-tagged jets, and large missing transverse energy, CMS-PAS-SUS-13-016.

[57] N. Arkani-Hamed, A. Gupta, D. E. Kaplan, N. Weiner, and T. Zorawski, Simply Unnatural Supersymmetry, arXiv:1212.6971.

[58] G. Giudice and A. Masiero, A Natural Solution to the mu Problem in Supergravity Theories, Phys.Lett. B206 (1988) 480-484.

[59] A. Gupta, D. E. Kaplan, and T. Zorawski, Gaugomaly Mediation Revisited, JHEP 1311 (2013) 149, [arXiv:1212.6969].

[60] T. Gherghetta, G. F. Giudice, and J. D. Wells, Phenomenological consequences of supersymmetry with anomaly induced masses, Nucl.Phys. B559 (1999) 27-47, [hep-ph/9904378].

[61] M. Picariello and A. Strumia, Next-to-leading order corrections to gauge mediated gaugino masses, Nucl.Phys. B529 (1998) 81-95, [hep-ph/9802446].

[62] J. L. Feng, T. Moroi, L. Randall, M. Strassler, and S.-f. Su, Discovering supersymmetry at the Tevatron in wino LSP scenarios, Phys.Rev.Lett. 83 (1999) 1731-1734, [hep-ph/9904250].

[63] M. Toharia and J. D. Wells, Gluino decays with heavier scalar superpartners, JHEP 0602 (2006) 015, [hep-ph/0503175].

[64] D. Buttazzo, G. Degrassi, P. P. Giardino, G. F. Giudice, F. Sala, et al., Investigating the near-criticality of the Higgs boson, JHEP 1312 (2013) 089, [arXiv: 1307.3536].

[65] L. N. Mihaila, J. Salomon, and M. Steinhauser, Renormalization constants and beta functions for the gauge couplings of the Standard Model to three-loop order, Phys.Rev. D86 (2012) 096008, [arXiv:1208.3357]. 
[66] M. Holthausen, K. S. Lim, and M. Lindner, Planck scale Boundary Conditions and the Higgs Mass, JHEP 1202 (2012) 037, [arXiv:1112.2415].

[67] G. F. Giudice and A. Strumia, Probing High-Scale and Split Supersymmetry with Higgs Mass Measurements, Nucl.Phys. B858 (2012) 63-83, [arXiv: 1108.6077].

[68] A. Sirlin and R. Zucchini, Dependence of the Quartic Coupling H(m) on $M(H)$ and the Possible Onset of New Physics in the Higgs Sector of the Standard Model, Nucl.Phys. B266 (1986) 389.

[69] M. Cabrera, J. Casas, and A. Delgado, Upper Bounds on Superpartner Masses from Upper Bounds on the Higgs Boson Mass, Phys.Rev.Lett. 108 (2012) 021802, [arXiv: 1108.3867].

[70] A. Bednyakov, A. Pikelner, and V. Velizhanin, Anomalous dimensions of gauge fields and gauge coupling beta-functions in the Standard Model at three loops, JHEP 1301 (2013) 017, [arXiv:1210.6873].

[71] A. Bednyakov, A. Pikelner, and V. Velizhanin, Yukawa coupling beta-functions in the Standard Model at three loops, Phys.Lett. B722 (2013) 336-340, [arXiv: 1212.6829$]$.

[72] A. Bednyakov, A. Pikelner, and V. Velizhanin, Higgs self-coupling beta-function in the Standard Model at three loops, Nucl.Phys. B875 (2013) 552-565, [arXiv:1303.4364].

[73] J. Barnard, B. Farmer, T. Gherghetta, and M. White, Natural gauge mediation with a bino NLSP at the LHC, Phys.Rev.Lett. 109 (2012) 241801, [arXiv: 1208.6062].

[74] J. Alwall, R. Frederix, S. Frixione, V. Hirschi, F. Maltoni, et al., The automated computation of tree-level and next-to-leading order differential cross sections, and their matching to parton shower simulations, JHEP 1407 (2014) 079, [arXiv: 1405.0301].

[75] T. Sjostrand, S. Mrenna, and P. Z. Skands, PYTHIA 6.4 Physics and Manual, JHEP 0605 (2006) 026, [hep-ph/0603175].

[76] DELPHES 3, J. de Favereau et al., DELPHES 3, A modular framework for fast simulation of a generic collider experiment, JHEP 1402 (2014) 057, [arXiv:1307.6346].

[77] M. Cacciari, G. P. Salam, and G. Soyez, FastJet User Manual, Eur. Phys. J. C72 (2012) 1896, [arXiv:1111.6097]. 
[78] M. Papucci, K. Sakurai, A. Weiler, and L. Zeune, Fastlim: a fast LHC limit calculator, Eur.Phys.J. C74 (2014), no. 11 3163, [arXiv:1402.0492].

[79] W. Beenakker, R. Hopker, M. Spira, and P. M. Zerwas, Squark and gluino production at hadron colliders, Nucl. Phys. B492 (1997) 51-103, [hep-ph/9610490].

[80] A. Kulesza and L. Motyka, Threshold resummation for squark-antisquark and gluino-pair production at the LHC, Phys.Rev.Lett. 102 (2009) 111802, [arXiv:0807.2405].

[81] A. Kulesza and L. Motyka, Soft gluon resummation for the production of gluino-gluino and squark-antisquark pairs at the LHC, Phys. Rev. D80 (2009) 095004, [arXiv:0905.4749].

[82] W. Beenakker, S. Brensing, M. Kramer, A. Kulesza, E. Laenen, et al., Soft-gluon resummation for squark and gluino hadroproduction, JHEP 0912 (2009) 041, [arXiv:0909.4418].

[83] W. Beenakker, S. Brensing, M. Kramer, A. Kulesza, E. Laenen, et al., Squark and Gluino Hadroproduction, Int.J.Mod.Phys. A26 (2011) 2637-2664, [arXiv: 1105.1110].

[84] W. Beenakker, R. Hopker, and M. Spira, PROSPINO: A Program for the production of supersymmetric particles in next-to-leading order $Q C D$, hep-ph/9611232.

[85] A. L. Read, Presentation of search results: The CL(s) technique, J.Phys. G28 (2002) 2693-2704.

[86] T. Junk, Confidence level computation for combining searches with small statistics, Nucl. Instrum. Meth. A434 (1999) 435-443, [hep-ex/9902006].

[87] T. A. W. Martin and D. Morrissey, Electroweakino constraints from LHC data, arXiv:1409.6322.

[88] CMS Collaboration, Study of the Discovery Reach in Searches for Supersymmetry at CMS with 3000/fb, CMS-PAS-FTR-13-014.

[89] T. Cohen, T. Golling, M. Hance, A. Henrichs, K. Howe, et al., SUSY Simplified Models at 14, 33, and 100 TeV Proton Colliders, JHEP 1404 (2014) 117, [arXiv:1311.6480].

[90] J. Anderson, A. Avetisyan, R. Brock, S. Chekanov, T. Cohen, et al., Snowmass Energy Frontier Simulations, arXiv:1309.1057. 
[91] A. Avetisyan, J. M. Campbell, T. Cohen, N. Dhingra, J. Hirschauer, et al., Methods and Results for Standard Model Event Generation at $\sqrt{\mathrm{s}}=14 \mathrm{TeV}$, $33 \mathrm{TeV}$ and $100 \mathrm{TeV}$ Proton Colliders (A Snowmass Whitepaper), arXiv: 1308.1636.

[92] I. Hinchliffe and F. Paige, High mass supersymmetry with high-energy hadron colliders, hep-ph/0201141.

[93] ATLAS, M. Aaboud et al., Search for supersymmetry in final states with missing transverse momentum and multiple b-jets in proton-proton collisions at $\sqrt{s}=13$ TeV with the ATLAS detector, JHEP 06 (2018) 107, [arXiv: 1711.01901].

[94] ATLAS, M. Aaboud et al., Search for top-squark pair production in final states with one lepton, jets, and missing transverse momentum using $36 \mathrm{fb}^{-1}$ of $\sqrt{s}=13$ TeV pp collision data with the ATLAS detector, JHEP 06 (2018) 108, [arXiv:1711.11520].

[95] CMS, A. M. Sirunyan et al., Search for natural and split supersymmetry in proton-proton collisions at $\sqrt{s}=13$ TeV in final states with jets and missing transverse momentum, JHEP 05 (2018) 025, [arXiv: 1802.02110].

[96] CMS, A. M. Sirunyan et al., Search for supersymmetry in proton-proton collisions at 13 TeV using identified top quarks, Phys. Rev. D97 (2018), no. 1 012007, [arXiv:1710.11188].

[97] ATLAS, M. Aaboud et al., Search for chargino-neutralino production using recursive jigsaw reconstruction in final states with two or three charged leptons in proton-proton collisions at $\sqrt{s}=13 \mathrm{TeV}$ with the ATLAS detector, Phys. Rev. D98 (2018), no. 9 092012, [arXiv:1806.02293].

[98] ATLAS, M. Aaboud et al., Search for chargino and neutralino production in final states with a Higgs boson and missing transverse momentum at $\sqrt{s}=13$ TeV with the ATLAS detector, Submitted to: Phys. Rev. (2018) [arXiv: 1812.09432].

[99] CMS, A. M. Sirunyan et al., Combined search for electroweak production of charginos and neutralinos in proton-proton collisions at $\sqrt{s}=13$ TeV, JHEP 03 (2018) 160, [arXiv: 1801.03957].

[100] ATLAS, G. Aad et al., Search for massive, long-lived particles using multitrack displaced vertices or displaced lepton pairs in pp collisions at $\sqrt{s}=$ 8 TeV with the ATLAS detector, Phys. Rev. D92 (2015), no. 7 072004, [arXiv: 1504.05162]. 
[101] CMS, A. M. Sirunyan et al., Search for long-lived particles with displaced vertices in multijet events in proton-proton collisions at $\sqrt{s}=13 \mathrm{TeV}$, Phys. Rev. D98 (2018), no. 9 092011, [arXiv:1808.03078].

[102] ATLAS, M. Aaboud et al., A search for pair-produced resonances in four-jet final states at $\sqrt{s}=13$ TeV with the ATLAS detector, Eur. Phys. J. C78 (2018), no. 3 250, [arXiv:1710.07171].

[103] ATLAS, M. Aaboud et al., Search for R-parity-violating supersymmetric particles in multi-jet final states produced in $p$-p collisions at $\sqrt{s}=13$ TeV using the ATLAS detector at the LHC, Phys. Lett. B785 (2018) 136-158, [arXiv: 1804.03568].

[104] CMS, V. Khachatryan et al., Searches for R-parity-violating supersymmetry in ppcollisions at $\sqrt{(s)}=8 \mathrm{TeV}$ in final states with 0-4 leptons, Phys. Rev. D94 (2016), no. 11 112009, [arXiv:1606.08076].

[105] ATLAS, M. Aaboud et al., Search for supersymmetry in events with four or more leptons in $\sqrt{s}=13$ TeV pp collisions with ATLAS, Phys. Rev. D98 (2018), no. 3 032009, [arXiv: 1804.03602].

[106] N.-E. Bomark, D. Choudhury, S. Lola, and P. Osland, Flavour Structure of R-violating Neutralino Decays at the LHC, JHEP 07 (2011) 070, [arXiv: 1105.4022].

[107] M. Asano, K. Rolbiecki, and K. Sakurai, Can R-parity violation hide vanilla supersymmetry at the LHC?, JHEP 01 (2013) 128, [arXiv:1209.5778].

[108] J. Berger, M. Perelstein, M. Saelim, and P. Tanedo, The Same-Sign Dilepton Signature of RPV/MFV SUSY, JHEP 04 (2013) 077, [arXiv:1302.2146].

[109] G. Durieux and C. Smith, The same-sign top signature of R-parity violation, JHEP 10 (2013) 068, [arXiv:1307.1355].

[110] A. Arvanitaki, M. Baryakhtar, X. Huang, K. van Tilburg, and G. Villadoro, The Last Vestiges of Naturalness, JHEP 03 (2014) 022, [arXiv:1309.3568].

[111] B. Bhattacherjee and A. Chakraborty, Study of the baryonic R-parity violating MSSM using the jet substructure technique at the 14 TeV LHC, Phys. Rev. D89 (2014), no. 11 115016, [arXiv:1311.5785].

[112] C. Faroughy, S. Prabhu, and B. Zheng, Simultaneous B and L Violation: New Signatures from RPV-SUSY, JHEP 06 (2015) 073, [arXiv:1409.5438].

[113] N.-E. Bomark, A. Kvellestad, S. Lola, P. Osland, and A. R. Raklev, R-parity violating chargino decays at the LHC, JHEP 12 (2014) 121, [arXiv: 1410.0921]. 
[114] D. Dercks, H. Dreiner, M. E. Krauss, T. Opferkuch, and A. Reinert, R-Parity Violation at the LHC, Eur. Phys. J. C77 (2017), no. 12 856, [arXiv: 1706.09418].

[115] S. Dumitru, B. A. Ovrut, and A. Purves, The R-parity Violating Decays of Charginos and Neutralinos in the B-L MSSM, JHEP 02 (2019) 124, [arXiv: 1810.11035].

[116] I. Lara, D. E. López-Fogliani, and C. Muñoz, Electroweak superpartners scrutinized at the LHC in events with multi-leptons, Phys. Lett. B790 (2019) 176-183, [arXiv:1810.12455].

[117] S. Dumitru, B. A. Ovrut, and A. Purves, R-parity Violating Decays of Wino Chargino and Wino Neutralino LSPs and NLSPs at the LHC, arXiv: 1811.05581.

[118] J. Cohen, S. Bar-Shalom, G. Eilam, and A. Soni, R-Parity Violating Supersymmetry and the 125 GeV Higgs signals, arXiv:1906.04743.

[119] J. Li, T. Li, and W. Zhang, Least constrained supersymmetry with R-parity violation, Phys. Rev. D99 (2019), no. 3 036011, [arXiv:1805.06172].

[120] S. Dumitru, C. Herwig, and B. A. Ovrut, R-parity Violating Decays of Bino Neutralino LSPs at the $L H C$, arXiv:1906.03174.

[121] S. Diglio, L. Feligioni, and G. Moultaka, Stashing the stops in multijet events at the LHC, Phys. Rev. D96 (2017), no. 5 055032, [arXiv:1611.05850].

[122] M. R. Buckley, D. Feld, S. Macaluso, A. Monteux, and D. Shih, Cornering Natural SUSY at LHC Run II and Beyond, JHEP 08 (2017) 115, [arXiv: 1610.08059].

[123] J. A. Evans and J. Shelton, Long-Lived Staus and Displaced Leptons at the LHC, JHEP 04 (2016) 056, [arXiv:1601.01326].

[124] E. J. Chun, S. Jung, H. M. Lee, and S. C. Park, Stop and Sbottom LSP with R-parity Violation, Phys. Rev. D90 (2014) 115023, [arXiv:1408.4508].

[125] M. Asano, K. Sakurai, and T. T. Yanagida, Multi-hadron final states in RPV supersymmetric models with extra matter, Phys. Lett. B736 (2014) 356-360, [arXiv: 1405.4009].

[126] Z. Marshall, B. A. Ovrut, A. Purves, and S. Spinner, LSP Squark Decays at the LHC and the Neutrino Mass Hierarchy, Phys. Rev. D90 (2014), no. 1 015034, [arXiv: 1402.5434]. 
[127] Z. Marshall, B. A. Ovrut, A. Purves, and S. Spinner, Spontaneous R-Parity Breaking, Stop LSP Decays and the Neutrino Mass Hierarchy, Phys. Lett. B732 (2014) 325-329, [arXiv:1401.7989].

[128] Y. Bai, A. Katz, and B. Tweedie, Pulling Out All the Stops: Searching for RPV SUSY with Stop-Jets, JHEP 01 (2014) 040, [arXiv: 1309.6631].

[129] R. Franceschini and R. Torre, RPV stops bump off the background, Eur. Phys. J. C73 (2013) 2422, [arXiv:1212.3622].

[130] Z. Han, A. Katz, M. Son, and B. Tweedie, Boosting searches for natural supersymmetry with R-parity violation via gluino cascades, Phys. Rev. D87 (2013), no. 7 075003, [arXiv:1211.4025].

[131] A. Monteux, New signatures and limits on R-parity violation from resonant squark production, JHEP 03 (2016) 216, [arXiv: 1601.03737].

[132] B. C. Allanach and B. Gripaios, Hide and Seek With Natural Supersymmetry at the LHC, JHEP 05 (2012) 062, [arXiv:1202.6616].

[133] J. A. Evans and Y. Kats, LHC Coverage of RPV MSSM with Light Stops, JHEP 04 (2013) 028, [arXiv:1209.0764].

[134] B. Bhattacherjee, J. L. Evans, M. Ibe, S. Matsumoto, and T. T. Yanagida, Natural supersymmetry?s last hope: R-parity violation via UDD operators, Phys. Rev. D87 (2013), no. 11 115002, [arXiv:1301.2336].

[135] P. W. Graham, S. Rajendran, and P. Saraswat, Supersymmetric crevices: Missing signatures of $R$-parity violation at the LHC, Phys. Rev. D90 (2014), no. 7 075005, [arXiv: 1403.7197].

[136] CMS, A. M. Sirunyan et al., Search for physics beyond the standard model in events with two leptons of same sign, missing transverse momentum, and jets in proton-proton collisions at $\sqrt{s}=13$ TeV, Eur. Phys. J. C77 (2017), no. 9 578, [arXiv:1704.07323].

[137] C. Csaki, Y. Grossman, and B. Heidenreich, MFV SUSY: A Natural Theory for R-Parity Violation, Phys. Rev. D85 (2012) 095009, [arXiv:1111.1239].

[138] G. F. Giudice, B. Gripaios, and R. Sundrum, Flavourful Production at Hadron Colliders, JHEP 08 (2011) 055, [arXiv:1105.3161].

[139] H. E. Haber, The Status of the minimal supersymmetric standard model and beyond, Nucl. Phys. Proc. Suppl. 62 (1998) 469-484, [hep-ph/9709450]. [,469(1997)]. 
[140] T.-F. Feng, L. Sun, and X.-Y. Yang, Electroweak and supersymmetric two-loop corrections to lepton anomalous magnetic and electric dipole moments, Nucl. Phys. B800 (2008) 221-252, [arXiv:0805.1122].

[141] J. R. Ellis, J. S. Lee, and A. Pilaftsis, Electric Dipole Moments in the MSSM Reloaded, JHEP 10 (2008) 049, [arXiv:0808.1819].

[142] K. Cheung, O. C. W. Kong, and J. S. Lee, Electric and anomalous magnetic dipole moments of the muon in the MSSM, JHEP 06 (2009) 020, [arXiv:0904.4352].

[143] W. Altmannshofer, A. J. Buras, S. Gori, P. Paradisi, and D. M. Straub, Anatomy and Phenomenology of FCNC and CPV Effects in SUSY Theories, Nucl. Phys. B830 (2010) 17-94, [arXiv:0909.1333].

[144] D. Pierce and A. Papadopoulos, Radiative corrections to neutralino and chargino masses in the minimal supersymmetric model, Phys. Rev. D50 (1994) 565-570, [hep-ph/9312248].

[145] D. Pierce and A. Papadopoulos, The Complete radiative corrections to the gaugino and Higgsino masses in the minimal supersymmetric model, Nucl. Phys. B430 (1994) 278-294, [hep-ph/9403240].

[146] A. B. Lahanas, K. Tamvakis, and N. D. Tracas, One loop corrections to the neutralino sector and radiative electroweak breaking in the MSSM, Phys. Lett. B324 (1994) 387-396, [hep-ph/9312251].

[147] G. F. Giudice and A. Pomarol, Mass degeneracy of the Higgsinos, Phys. Lett. B372 (1996) 253-258, [hep-ph/9512337].

[148] M. Drees, M. M. Nojiri, D. P. Roy, and Y. Yamada, Light Higgsino dark matter, Phys. Rev. D56 (1997) 276-290, [hep-ph/9701219]. [Erratum: Phys. Rev.D64,039901(2001)].

[149] G. Passarino and M. J. G. Veltman, One Loop Corrections for e+ eAnnihilation Into mu+ mu- in the Weinberg Model, Nucl. Phys. B160 (1979) $151-207$.

[150] A. Denner, Techniques for calculation of electroweak radiative corrections at the one loop level and results for W physics at LEP-200, Fortsch. Phys. 41 (1993) 307-420, [arXiv:0709.1075].

[151] T. Hahn and M. Perez-Victoria, Automatized one loop calculations in four-dimensions and D-dimensions, Comput. Phys. Commun. 118 (1999) 153-165, [hep-ph/9807565]. 
[152] G. J. van Oldenborgh and J. A. M. Vermaseren, New Algorithms for One Loop Integrals, Z. Phys. C46 (1990) 425-438.

[153] C. H. Chen, M. Drees, and J. F. Gunion, A Nonstandard string / SUSY scenario and its phenomenological implications, Phys. Rev. D55 (1997) 330-347, [hep-ph/9607421]. [Erratum: Phys. Rev.D60,039901(1999)].

[154] T. Sjöstrand, S. Ask, J. R. Christiansen, R. Corke, N. Desai, P. Ilten, S. Mrenna, S. Prestel, C. O. Rasmussen, and P. Z. Skands, An Introduction to PYTHIA 8.2, Comput. Phys. Commun. 191 (2015) 159-177, [arXiv: 1410.3012].

[155] J. M. Frere, D. R. T. Jones, and S. Raby, Fermion Masses and Induction of the Weak Scale by Supergravity, Nucl. Phys. B222 (1983) 11-19.

[156] J. P. Derendinger and C. A. Savoy, Quantum Effects and SU(2) $x$ U(1) Breaking in Supergravity Gauge Theories, Nucl. Phys. B237 (1984) 307-328.

[157] C. Kounnas, A. B. Lahanas, D. V. Nanopoulos, and M. Quiros, Low-Energy Behavior of Realistic Locally Supersymmetric Grand Unified Theories, Nucl. Phys. B236 (1984) 438-466.

[158] J. A. Casas, A. Lleyda, and C. Munoz, Strong constraints on the parameter space of the MSSM from charge and color breaking minima, Nucl. Phys. B471 (1996) 3-58, [hep-ph/9507294].

[159] Z. Liu and B. Tweedie, The Fate of Long-Lived Superparticles with Hadronic Decays after LHC Run 1, JHEP 06 (2015) 042, [arXiv: 1503.05923].

[160] P. Fayet, Massive Gluinos, Phys. Lett. B78 (1978) 417-420.

[161] L. J. Hall and L. Randall, U(1)-R symmetric supersymmetry, Nucl. Phys. B352 (1991) 289-308.

[162] P. J. Fox, A. E. Nelson, and N. Weiner, Dirac gaugino masses and supersoft supersymmetry breaking, JHEP 08 (2002) 035, [hep-ph/0206096].

[163] A. E. Nelson, N. Rius, V. Sanz, and M. Unsal, The Minimal supersymmetric model without a mu term, JHEP 08 (2002) 039, [hep-ph/0206102].

[164] G. D. Kribs, E. Poppitz, and N. Weiner, Flavor in supersymmetry with an extended R-symmetry, Phys. Rev. D78 (2008) 055010, [arXiv:0712.2039].

[165] S. D. L. Amigo, A. E. Blechman, P. J. Fox, and E. Poppitz, R-symmetric gauge mediation, JHEP 01 (2009) 018, [arXiv:0809.1112]. 
[166] K. Benakli and M. D. Goodsell, Dirac Gauginos in General Gauge Mediation, Nucl. Phys. B816 (2009) 185-203, [arXiv:0811.4409].

[167] K. Benakli and M. D. Goodsell, Dirac Gauginos, Gauge Mediation and Unification, Nucl. Phys. B840 (2010) 1-28, [arXiv:1003.4957].

[168] G. D. Kribs, T. Okui, and T. S. Roy, Viable Gravity-Mediated Supersymmetry Breaking, Phys. Rev. D82 (2010) 115010, [arXiv: 1008.1798].

[169] S. Abel and M. Goodsell, Easy Dirac Gauginos, JHEP 06 (2011) 064, [arXiv: 1102.0014].

[170] C. Csaki, J. Goodman, R. Pavesi, and Y. Shirman, The $m_{D}-b_{M}$ problem of Dirac gauginos and its solutions, Phys. Rev. D89 (2014), no. 5 055005, [arXiv: 1310.4504].

[171] R. Davies, J. March-Russell, and M. McCullough, A Supersymmetric One Higgs Doublet Model, JHEP 04 (2011) 108, [arXiv:1103.1647].

[172] C. Frugiuele and T. Gregoire, Making the Sneutrino a Higgs with a $U(1)_{R}$ Lepton Number, Phys. Rev. D85 (2012) 015016, [arXiv:1107.4634].

[173] R. Fok, G. D. Kribs, A. Martin, and Y. Tsai, Electroweak Baryogenesis in R-symmetric Supersymmetry, Phys. Rev. D87 (2013), no. 5 055018, [arXiv: 1208.2784].

[174] C. Frugiuele, T. Gregoire, P. Kumar, and E. Ponton, ' $L=R$ ' $-U(1)_{R}$ Lepton Number at the LHC, JHEP 05 (2013) 012, [arXiv: 1210.5257].

[175] C. Frugiuele, T. Gregoire, P. Kumar, and E. Ponton, ' $L=R$ ' - $U(1)_{R}$ as the Origin of Leptonic 'RPV', JHEP 03 (2013) 156, [arXiv:1210.0541].

[176] H. Beauchesne and T. Gregoire, Electroweak precision measurements in supersymmetric models with a $U(1)_{R}$ lepton number, JHEP 05 (2014) 051, [arXiv: 1402.5403].

[177] E. Bertuzzo, C. Frugiuele, T. Gregoire, and E. Ponton, Dirac gauginos, $R$ symmetry and the 125 GeV Higgs, JHEP 04 (2015) 089, [arXiv:1402.5432].

[178] L. M. Carpenter and J. Goodman, New Calculations in Dirac Gaugino Models: Operators, Expansions, and Effects, JHEP 07 (2015) 107, [arXiv: 1501.05653].

[179] H. Itoyama and N. Maru, D-term Dynamical Supersymmetry Breaking Generating Split N=2 Gaugino Masses of Mixed Majorana-Dirac Type, Int. J. Mod. Phys. A27 (2012) 1250159, [arXiv:1109.2276]. 
[180] H. Itoyama and N. Maru, D-term Triggered Dynamical Supersymmetry Breaking, Phys. Rev. D88 (2013), no. 2 025012, [arXiv:1301.7548].

[181] H. Itoyama and N. Maru, 126 GeV Higgs Boson Associated with D-term Triggered Dynamical Supersymmetry Breaking, Symmetry 7 (2015), no. 1 193-205, [arXiv: 1312.4157].

[182] M. Heikinheimo, M. Kellerstein, and V. Sanz, How Many Supersymmetries?, JHEP 04 (2012) 043, [arXiv: 1111.4322].

[183] G. D. Kribs and A. Martin, Supersoft Supersymmetry is Super-Safe, Phys. Rev. D85 (2012) 115014, [arXiv:1203.4821].

[184] G. D. Kribs and N. Raj, Mixed Gauginos Sending Mixed Messages to the LHC, Phys. Rev. D89 (2014), no. 5 055011, [arXiv:1307.7197].

[185] R. Fok and G. D. Kribs, $\mu$ to e in R-symmetric Supersymmetry, Phys. Rev. D82 (2010) 035010, [arXiv:1004.0556].

[186] T. Gherghetta and A. Pomarol, The Standard model partly supersymmetric, Phys. Rev. D67 (2003) 085018, [hep-ph/0302001].

[187] F. Riva, C. Biggio, and A. Pomarol, Is the 125 GeV Higgs the superpartner of a neutrino?, JHEP 02 (2013) 081, [arXiv: 1211.4526].

[188] C. Biggio, J. A. Dror, Y. Grossman, and W. H. Ng, Probing a slepton Higgs on all frontiers, JHEP 04 (2016) 150, [arXiv:1602.02162].

[189] C. Brust, A. Katz, S. Lawrence, and R. Sundrum, SUSY, the Third Generation and the LHC, JHEP 03 (2012) 103, [arXiv:1110.6670].

[190] H. K. Dreiner and G. G. Ross, R-parity violation at hadron colliders, Nucl. Phys. B365 (1991) 597-613.

[191] Super-Kamiokande, K. Abe et al., The Search for $n-\bar{n}$ oscillation in Super-Kamiokande I, Phys. Rev. D91 (2015) 072006, [arXiv:1109.4227].

[192] M. Litos et al., Search for Dinucleon Decay into Kaons in Super-Kamiokande, Phys. Rev. Lett. 112 (2014), no. 13131803.

[193] J. L. Goity and M. Sher, Bounds on $\Delta B=1$ couplings in the supersymmetric standard model, Phys. Lett. B346 (1995) 69-74, [hep-ph/9412208]. [Erratum: Phys. Lett.B385,500(1996)].

[194] A. De Simone, V. Sanz, and H. P. Sato, Pseudo-Dirac Dark Matter Leaves a Trace, Phys. Rev. Lett. 105 (2010) 121802, [arXiv:1004.1567]. 
[195] S. Ipek and J. March-Russell, Baryogenesis via Particle-Antiparticle Oscillations, Phys. Rev. D93 (2016), no. 12 123528, [arXiv: 1604.00009].

[196] E. L. Berger, B. W. Harris, and Z. Sullivan, Single top squark production via $R$-parity violating supersymmetric couplings in hadron collisions, Phys. Rev. Lett. 83 (1999) 4472-4475, [hep-ph/9903549].

[197] A. Alloul, N. D. Christensen, C. Degrande, C. Duhr, and B. Fuks, FeynRules 2.0 - A complete toolbox for tree-level phenomenology, Comput. Phys. Commun. 185 (2014) 2250-2300, [arXiv:1310.1921].

[198] T. Plehn, Single stop production at hadron colliders, Phys. Lett. B488 (2000) 359-366, [hep-ph/0006182].

[199] W. Beenakker, M. Kramer, T. Plehn, M. Spira, and P. M. Zerwas, Stop production at hadron colliders, Nucl. Phys. B515 (1998) 3-14, [hep-ph/9710451].

[200] W. Beenakker, S. Brensing, M. Kramer, A. Kulesza, E. Laenen, and I. Niessen, Supersymmetric top and bottom squark production at hadron colliders, JHEP 08 (2010) 098, [arXiv:1006.4771].

[201] W. Beenakker, C. Borschensky, M. Kramer, A. Kulesza, and E. Laenen, NNLL-fast: predictions for coloured supersymmetric particle production at the LHC with threshold and Coulomb resummation, JHEP 12 (2016) 133, [arXiv: 1607.07741].

[202] W. Beenakker, C. Borschensky, R. Heger, M. Kramer, A. Kulesza, and E. Laenen, NNLL resummation for stop pair-production at the LHC, JHEP 05 (2016) 153, [arXiv:1601.02954].

[203] ATLAS, G. Aad et al., Search for new phenomena in the dijet mass distribution using $p-p$ collision data at $\sqrt{s}=8$ TeV with the ATLAS detector, Phys. Rev. D91 (2015), no. 5 052007, [arXiv:1407.1376].

[204] ATLAS Collaboration, Search for light dijet resonances with the ATLAS detector using a Trigger-Level Analysis in LHC pp collisions at $\sqrt{s}=13$ TeV, ATLAS-CONF-2016-030, CERN, Geneva, Jun, 2016.

[205] ATLAS Collaboration, Search for New Phenomena in Dijet Events with the ATLAS Detector at $\sqrt{s}=13$ TeV with 2015 and 2016 data, ATLAS-CONF-2016-069, CERN, Geneva, Aug, 2016.

[206] CMS, V. Khachatryan et al., Search for narrow resonances in dijet final states at $\sqrt{(} s)=8$ TeV with the novel CMS technique of data scouting, Phys. Rev. Lett. 117 (2016), no. 3 031802, [arXiv:1604.08907]. 
[207] CMS Collaboration, Searches for narrow resonances decaying to dijets in proton-proton collisions at 13 TeV using 12.9 inverse femtobarns., CMS-PAS-EXO-16-032, CERN, Geneva, 2016.

[208] ATLAS Collaboration, Search for resonances in the mass distribution of jet pairs with one or two jets identified as b-jets with the ATLAS detector with 2015 and 2016 data, ATLAS-CONF-2016-060, CERN, Geneva, Aug, 2016.

[209] M. Dobbs and J. B. Hansen, The HepMC C++ Monte Carlo event record for High Energy Physics, Comput. Phys. Commun. 134 (2001) 41-46.

[210] ATLAS, G. Aad et al., A search for top squarks with R-parity-violating decays to all-hadronic final states with the ATLAS detector in $\sqrt{s}=8$ TeV proton-proton collisions, JHEP 06 (2016) 067, [arXiv:1601.07453].

[211] ATLAS Collaboration, A search for R-parity violating decays of the top squark in four jet final states with the ATLAS detector at $\sqrt{s}=13 \mathrm{TeV}$, ATLAS-CONF-2016-022, CERN, Geneva, May, 2016.

[212] ATLAS Collaboration, A search for pair produced resonances in four jets final states in proton-proton collisions at $\sqrt{s}=13$ TeV with the ATLAS experiment, ATLAS-CONF-2016-084, CERN, Geneva, Aug, 2016.

[213] CMS, V. Khachatryan et al., Search for pair-produced resonances decaying to jet pairs in proton-proton collisions at $\sqrt{s}=8$ TeV, Phys. Lett. B747 (2015) 98-119, [arXiv:1412.7706].

[214] ATLAS Collaboration, Search for supersymmetry with two same-sign leptons or three leptons using 13.2 $\mathrm{fb}^{-1}$ of $\sqrt{\mathrm{s}}=13 \mathrm{TeV}$ pp collision data collected by the ATLAS detector, ATLAS-CONF-2016-037, CERN, Geneva, Aug, 2016.

[215] ATLAS Collaboration, Search for massive supersymmetric particles in multi-jet final states produced in pp collisions at $\sqrt{s}=13$ TeV using the ATLAS detector at the LHC, ATLAS-CONF-2016-057, CERN, Geneva, Aug, 2016.

[216] ATLAS Collaboration, Search for new physics in a lepton plus high jet multiplicity final state with the ATLAS experiment using $\operatorname{sqrt}(\mathrm{s})=13 \mathrm{TeV}$ proton-proton collision data, ATLAS-CONF-2016-094, CERN, Geneva, Aug, 2016.

[217] ATLAS Collaboration, Search for production of vector-like top quark pairs and of four top quarks in the lepton-plus-jets final state in pp collisions at $\sqrt{s}=13$ TeV with the ATLAS detector, ATLAS-CONF-2016-013, CERN, Geneva, Mar, 2016. 
[218] ATLAS Collaboration, Search for new physics using events with b-jets and a pair of same charge leptons in 3.2 $\mathrm{fb}^{-1}$ of pp collisions at $\sqrt{s}=13$ TeV with the ATLAS detector, ATLAS-CONF-2016-032, CERN, Geneva, Jun, 2016.

[219] CMS Collaboration, Search for SUSY in same-sign dilepton events at 13 TeV, CMS-PAS-SUS-16-020, CERN, Geneva, 2016.

[220] M. Cacciari and G. P. Salam, Dispelling the $N^{3}$ myth for the $k_{t}$ jet-finder, Phys. Lett. B641 (2006) 57-61, [hep-ph/0512210].

[221] Y. Cui and B. Shuve, Probing Baryogenesis with Displaced Vertices at the LHC, JHEP 02 (2015) 049, [arXiv: 1409.6729].

[222] CMS, V. Khachatryan et al., Search for Long-Lived Neutral Particles Decaying to Quark-Antiquark Pairs in Proton-Proton Collisions at $\sqrt{s}=8$ TeV, Phys. Rev. D91 (2015), no. 1 012007, [arXiv:1411.6530].

[223] W. Beenakker, S. Brensing, M. Kramer, A. Kulesza, E. Laenen, and I. Niessen, NNLL resummation for squark-antisquark pair production at the LHC, JHEP 01 (2012) 076, [arXiv:1110.2446].

[224] W. Beenakker, T. Janssen, S. Lepoeter, M. Krämer, A. Kulesza, E. Laenen, I. Niessen, S. Thewes, and T. Van Daal, Towards NNLL resummation: hard matching coefficients for squark and gluino hadroproduction, JHEP 10 (2013) 120, [arXiv:1304.6354].

[225] W. Beenakker, C. Borschensky, M. Krämer, A. Kulesza, E. Laenen, $\mathrm{V}$. Theeuwes, and S. Thewes, NNLL resummation for squark and gluino production at the LHC, JHEP 12 (2014) 023, [arXiv:1404.3134].

[226] ATLAS Collaboration, Further searches for squarks and gluinos in final states with jets and missing transverse momentum at $\sqrt{s}=13$ TeV with the ATLAS detector, ATLAS-CONF-2016-078, CERN, Geneva, Aug, 2016.

[227] K. Benakli, M. D. Goodsell, and F. Staub, Dirac Gauginos and the 125 GeV Higgs, JHEP 06 (2013) 073, [arXiv: 1211.0552].

[228] P. Dießner, J. Kalinowski, W. Kotlarski, and D. Stöckinger, Higgs boson mass and electroweak observables in the MRSSM, JHEP 12 (2014) 124, [arXiv: 1410.4791].

[229] LHCb, R. Aaij et al., Test of lepton universality using $B^{+} \rightarrow K^{+} \ell^{+} \ell^{-}$decays, Phys. Rev. Lett. 113 (2014) 151601, [arXiv:1406.6482].

[230] M. Bordone, G. Isidori, and A. Pattori, On the Standard Model predictions for $R_{K}$ and $R_{K^{*}}$, Eur. Phys. J. C76 (2016), no. 8 440, [arXiv:1605.07633]. 
[231] C. Bobeth, G. Hiller, and G. Piranishvili, Angular distributions of $\bar{B} \rightarrow \bar{K} \ell^{+} \ell^{-}$decays, JHEP 12 (2007) 040, [arXiv:0709.4174].

[232] LHCb, R. Aaij et al., Test of lepton universality with $B^{0} \rightarrow K^{* 0} \ell^{+} \ell^{-}$decays, JHEP 08 (2017) 055, [arXiv:1705.05802].

[233] J. Matias, F. Mescia, M. Ramon, and J. Virto, Complete Anatomy of $\bar{B}_{d}->\bar{K}^{* 0}(->K \pi) l^{+} l^{-}$and its angular distribution, JHEP 04 (2012) 104, [arXiv:1202.4266].

[234] S. Descotes-Genon, T. Hurth, J. Matias, and J. Virto, Optimizing the basis of $B \rightarrow K^{*} l l$ observables in the full kinematic range, JHEP 05 (2013) 137, [arXiv: 1303.5794].

[235] S. Descotes-Genon, J. Matias, M. Ramon, and J. Virto, Implications from clean observables for the binned analysis of $B->K * \mu^{+} \mu^{-}$at large recoil, JHEP 01 (2013) 048, [arXiv:1207.2753].

[236] Belle, S. Wehle et al., Lepton-Flavor-Dependent Angular Analysis of $B \rightarrow K^{*} \ell^{+} \ell^{-}$, Phys. Rev. Lett. 118 (2017), no. 11 111801, [arXiv: 1612.05014].

[237] Belle, A. Abdesselam et al., Angular analysis of $B^{0} \rightarrow K^{*}(892)^{0} \ell^{+} \ell^{-}$, in Proceedings, LHCSki 2016 - A First Discussion of 13 TeV Results: Obergurgl, Austria, April 10-15, 2016, 2016. arXiv:1604.04042.

[238] LHCb, R. Aaij et al., Angular analysis of the $B^{0} \rightarrow K^{* 0} \mu^{+} \mu^{-}$decay using 3 $\mathrm{fb}^{-1}$ of integrated luminosity, JHEP 02 (2016) 104, [arXiv:1512.04442].

[239] LHCb, R. Aaij et al., Measurement of Form-Factor-Independent Observables in the Decay $B^{0} \rightarrow K^{* 0} \mu^{+} \mu^{-}$, Phys. Rev. Lett. 111 (2013) 191801, [arXiv: 1308.1707].

[240] B. Capdevila, A. Crivellin, S. Descotes-Genon, J. Matias, and J. Virto, Patterns of New Physics in $b \rightarrow s \ell^{+} \ell^{-}$transitions in the light of recent data, JHEP 01 (2018) 093, [arXiv: 1704.05340].

[241] LHCb, R. Aaij et al., Angular analysis and differential branching fraction of the decay $B_{s}^{0} \rightarrow \phi \mu^{+} \mu^{-}$, JHEP 09 (2015) 179, [arXiv:1506.08777].

[242] LHCb, R. Aaij et al., Differential branching fraction and angular analysis of the decay $B_{s}^{0} \rightarrow \phi \mu^{+} \mu^{-}$, JHEP 07 (2013) 084, [arXiv:1305.2168].

[243] S. Descotes-Genon, J. Matias, and J. Virto, Understanding the $B \rightarrow K^{*} \mu^{+} \mu^{-}$ Anomaly, Phys. Rev. D88 (2013) 074002, [arXiv:1307.5683]. 
[244] G. Hiller and M. Schmaltz, Diagnosing lepton-nonuniversality in $b \rightarrow$ sll, JHEP 02 (2015) 055, [arXiv:1411.4773].

[245] W. Altmannshofer and D. M. Straub, New physics in $b \rightarrow$ s transitions after LHC run 1, Eur. Phys. J. C75 (2015), no. 8 382, [arXiv:1411.3161].

[246] S. Descotes-Genon, L. Hofer, J. Matias, and J. Virto, Global analysis of $b \rightarrow$ sll anomalies, JHEP 06 (2016) 092, [arXiv: 1510.04239].

[247] W. Altmannshofer, P. Stangl, and D. M. Straub, Interpreting Hints for Lepton Flavor Universality Violation, Phys. Rev. D96 (2017), no. 5 055008, [arXiv: 1704.05435].

[248] D. Bardhan, P. Byakti, and D. Ghosh, Role of Tensor operators in $R_{K}$ and $R_{K^{*}}$, Phys. Lett. B773 (2017) 505-512, [arXiv:1705.09305].

[249] D. Ghosh, Explaining the $R_{K}$ and $R_{K^{*}}$ anomalies, Eur. Phys. J. C77 (2017), no. 10 694, [arXiv:1704.06240].

[250] BaBar, J. P. Lees et al., Evidence for an excess of $\bar{B} \rightarrow D^{(*)} \tau^{-} \bar{\nu}_{\tau}$ decays, Phys. Rev. Lett. 109 (2012) 101802, [arXiv:1205.5442].

[251] BaBar, J. P. Lees et al., Measurement of an Excess of $\bar{B} \rightarrow D^{(*)} \tau^{-} \bar{\nu}_{\tau}$ Decays and Implications for Charged Higgs Bosons, Phys. Rev. D88 (2013), no. 7 072012, [arXiv: 1303.0571].

[252] Belle, M. Huschle et al., Measurement of the branching ratio of $\bar{B} \rightarrow D^{(*)} \tau^{-} \bar{\nu}_{\tau}$ relative to $\bar{B} \rightarrow D^{(*)} \ell^{-} \bar{\nu}_{\ell}$ decays with hadronic tagging at Belle, Phys. Rev. D92 (2015), no. 7 072014, [arXiv:1507.03233].

[253] Belle, Y. Sato et al., Measurement of the branching ratio of $\bar{B}^{0} \rightarrow D^{*+} \tau^{-} \bar{\nu}_{\tau}$ relative to $\bar{B}^{0} \rightarrow D^{*+} \ell^{-} \bar{\nu}_{\ell}$ decays with a semileptonic tagging method, Phys. Rev. D94 (2016), no. 7 072007, [arXiv:1607.07923].

[254] A. Abdesselam et al., Measurement of the $\tau$ lepton polarization in the decay $\bar{B} \rightarrow D^{*} \tau^{-} \bar{\nu}_{\tau}$, arXiv $: 1608.06391$.

[255] Belle, S. Hirose et al., Measurement of the $\tau$ lepton polarization and $R\left(D^{*}\right)$ in the decay $\bar{B} \rightarrow D^{*} \tau^{-} \bar{\nu}_{\tau}$, Phys. Rev. Lett. 118 (2017), no. 21211801 , [arXiv: 1612.00529].

[256] Belle, S. Hirose et al., Measurement of the $\tau$ lepton polarization and $R\left(D^{*}\right)$ in the decay $\bar{B} \rightarrow D^{*} \tau^{-} \bar{\nu}_{\tau}$ with one-prong hadronic $\tau$ decays at Belle, Phys. Rev. D97 (2018), no. 1 012004, [arXiv: 1709.00129]. 
[257] LHCb, R. Aaij et al., Measurement of the ratio of branching fractions $\mathcal{B}\left(\bar{B}^{0} \rightarrow D^{*+} \tau^{-} \bar{\nu}_{\tau}\right) / \mathcal{B}\left(\bar{B}^{0} \rightarrow D^{*+} \mu^{-} \bar{\nu}_{\mu}\right)$, Phys. Rev. Lett. 115 (2015), no. 11 111803, [arXiv:1506.08614]. [Erratum: Phys. Rev. Lett.115,no.15,159901(2015)].

[258] HFLAV, Y. Amhis et al., Averages of b-hadron, c-hadron, and $\tau$-lepton properties as of summer 2016, Eur. Phys. J. C77 (2017), no. 12 895, [arXiv: 1612.07233].

[259] Y. Sakaki, M. Tanaka, A. Tayduganov, and R. Watanabe, Testing leptoquark models in $\bar{B} \rightarrow D^{(*)} \tau \bar{\nu}$, Phys. Rev. D88 (2013), no. 9094012 , [arXiv: 1309.0301].

[260] G. Hiller and M. Schmaltz, $R_{K}$ and future $b \rightarrow$ sll physics beyond the standard model opportunities, Phys. Rev. D90 (2014) 054014, [arXiv:1408.1627].

[261] M. Freytsis, Z. Ligeti, and J. T. Ruderman, Flavor models for $\bar{B} \rightarrow D^{(*)} \tau \bar{\nu}$, Phys. Rev. D92 (2015), no. 5 054018, [arXiv: 1506.08896].

[262] M. Bauer and M. Neubert, Minimal Leptoquark Explanation for the $R_{D^{(*)}}$, $R_{K}$, and $(g-2)_{g}$ Anomalies, Phys. Rev. Lett. 116 (2016), no. 14 141802, [arXiv:1511.01900].

[263] B. Allanach, F. S. Queiroz, A. Strumia, and S. Sun, Z' models for the LHCb and $g-2$ muon anomalies, Phys. Rev. D93 (2016), no. 5 055045, [arXiv: 1511.07447]. [Erratum: Phys. Rev.D95,no.11,119902(2017)].

[264] I. Doršner, S. Fajfer, A. Greljo, J. F. Kamenik, and N. Košnik, Physics of leptoquarks in precision experiments and at particle colliders, Phys. Rept. $\mathbf{6 4 1}$ (2016) 1-68, [arXiv:1603.04993].

[265] D. Das, C. Hati, G. Kumar, and N. Mahajan, Towards a unified explanation of $R_{D^{(*)}}, R_{K}$ and $(g-2)_{\mu}$ anomalies in a left-right model with leptoquarks, Phys. Rev. D94 (2016) 055034, [arXiv:1605.06313].

[266] D. Be?irevi?, N. Košnik, O. Sumensari, and R. Zukanovich Funchal, Palatable Leptoquark Scenarios for Lepton Flavor Violation in Exclusive $b \rightarrow s \ell_{1} \ell_{2}$ modes, JHEP 11 (2016) 035, [arXiv:1608.07583].

[267] D. Bečirević, S. Fajfer, N. Košnik, and O. Sumensari, Leptoquark model to explain the B-physics anomalies, $R_{K}$ and $R_{D}$, Phys. Rev. D94 (2016), no. 11 115021, [arXiv: 1608.08501].

[268] G. Hiller, D. Loose, and K. Schönwald, Leptoquark Flavor Patterns 83 B Decay Anomalies, JHEP 12 (2016) 027, [arXiv: 1609.08895]. 
[269] D. Bečirević and O. Sumensari, A leptoquark model to accommodate $R_{K}^{\exp }<R_{K}^{\mathrm{SM}}$ and $R_{K^{*}}^{\exp }<R_{K^{*}}^{\mathrm{SM}}, J H E P 08$ (2017) 104, [arXiv: 1704.05835].

[270] A. K. Alok, D. Kumar, J. Kumar, and R. Sharma, Lepton flavor non-universality in the B-sector: a global analyses of various new physics models, arXiv: 1704.07347.

[271] A. K. Alok, B. Bhattacharya, A. Datta, D. Kumar, J. Kumar, and D. London, New Physics in $b \rightarrow s \mu^{+} \mu^{-}$after the Measurement of $R_{K^{*}}$, Phys. Rev. D96 (2017), no. 9 095009, [arXiv:1704.07397].

[272] D. Aloni, A. Dery, C. Frugiuele, and Y. Nir, Testing minimal flavor violation in leptoquark models of the $R_{K^{(*)}}$ anomaly, JHEP 11 (2017) 109, [arXiv: 1708.06161].

[273] N. Assad, B. Fornal, and B. Grinstein, Baryon Number and Lepton Universality Violation in Leptoquark and Diquark Models, Phys. Lett. B777 (2018) 324-331, [arXiv: 1708.06350].

[274] L. Calibbi, A. Crivellin, and T. Li, A model of vector leptoquarks in view of the B-physics anomalies, arXiv:1709.00692.

[275] S. Biswas, D. Chowdhury, S. Han, and S. J. Lee, Explaining the lepton non-universality at the $\mathrm{LHCb}$ and CMS within a unified framework, JHEP 02 (2015) 142, [arXiv: 1409.0882].

[276] D. Das, C. Hati, G. Kumar, and N. Mahajan, Scrutinizing R-parity violating interactions in light of $R_{K^{(*)}}$ data, Phys. Rev. D96 (2017), no. 9 095033, [arXiv:1705.09188].

[277] N. G. Deshpande and A. Menon, Hints of R-parity violation in B decays into $\tau \nu$, JHEP 01 (2013) 025, [arXiv: 1208.4134].

[278] N. G. Deshpande and X.-G. He, Consequences of R-parity violating interactions for anomalies in $\bar{B} \rightarrow D^{(*)} \tau \bar{\nu}$ and $b \rightarrow s \mu^{+} \mu^{-}$, Eur. Phys. J. C77 (2017), no. 2 134, [arXiv: 1608.04817].

[279] W. Altmannshofer, P. Bhupal Dev, and A. Soni, $R_{D^{(*)}}$ anomaly: A possible hint for natural supersymmetry with $R$-parity violation, Phys. Rev. D96 (2017), no. 9 095010, [arXiv:1704.06659].

[280] A. de Gouvea, S. Lola, and K. Tobe, Lepton flavor violation in supersymmetric models with trilinear R-parity violation, Phys. Rev. D63 (2001) 035004, [hep-ph/0008085].

[281] B. de Carlos and P. L. White, R-parity violation and quark flavor violation, Phys. Rev. D55 (1997) 4222-4239, [hep-ph/9609443]. 
[282] T. Besmer and A. Steffen, R-parity violation and the decay $b-i$ s gamma, Phys. Rev. D63 (2001) 055007, [hep-ph/0004067].

[283] J. E. Kim, P. Ko, and D.-G. Lee, More on R-parity and lepton family number violating couplings from muon(ium) conversion, and tau and piO decays, Phys. Rev. D56 (1997) 100-106, [hep-ph/9701381].

[284] Particle Data Group, C. Patrignani et al., Review of Particle Physics, Chin. Phys. C40 (2016), no. 10100001.

[285] R. Mertig, M. Bohm, and A. Denner, FEYN CALC: Computer algebraic calculation of Feynman amplitudes, Comput. Phys. Commun. 64 (1991) 345-359.

[286] V. Shtabovenko, R. Mertig, and F. Orellana, New Developments in FeynCalc 9.0, Comput. Phys. Commun. 207 (2016) 432-444, [arXiv:1601.01167].

[287] UTfit, M. Bona et al., Model-independent constraints on $\Delta F=2$ operators and the scale of new physics, JHEP 03 (2008) 049, [arXiv:0707.0636].

[288] Belle, J. Grygier et al., Search for $\boldsymbol{B} \rightarrow \boldsymbol{h} \boldsymbol{\nu} \overline{\boldsymbol{\nu}}$ decays with semileptonic tagging at Belle, Phys. Rev. D96 (2017), no. 9 091101, [arXiv:1702.03224].

[289] ATLAS, M. Aaboud et al., Search for $B-L R$-parity-violating top squarks in $\sqrt{s}=13$ TeV pp collisions with the ATLAS experiment, Phys. Rev. D97 (2018), no. 3 032003, [arXiv:1710.05544].

[290] CMS, A. M. Sirunyan et al., Search for third-generation scalar leptoquarks and heavy right-handed neutrinos in final states with two tau leptons and two jets in proton-proton collisions at $\sqrt{s}=13$ TeV, JHEP 07 (2017) 121, [arXiv: 1703.03995].

[291] B. C. Allanach, A. Dedes, and H. K. Dreiner, Two loop supersymmetric renormalization group equations including $R$-parity violation and aspects of unification, Phys. Rev. D60 (1999) 056002, [hep-ph/9902251]. [Erratum: Phys. Rev.D86,039906(2012)].

[292] CMS Collaboration, Constraints on models of scalar and vector leptoquarks decaying to a quark and a neutrino at $\sqrt{s}=13 \mathrm{TeV}$, CMS-PAS-SUS-18-001, CERN, Geneva, 2018.

[293] C. Bobeth, M. Gorbahn, T. Hermann, M. Misiak, E. Stamou, and M. Steinhauser, $B_{s, d} \rightarrow l^{+} l^{-}$in the Standard Model with Reduced Theoretical Uncertainty, Phys. Rev. Lett. 112 (2014) 101801, [arXiv:1311.0903]. 
[294] C. Bobeth, Updated $B_{q} \rightarrow \bar{\ell} \ell$ in the standard model at higher orders, in Proceedings, 49th Rencontres de Moriond on Electroweak Interactions and Unified Theories: La Thuile, Italy, March 15-22, 2014, pp. 75-80, 2014. arXiv: 1405.4907.

[295] D. Bečirević, O. Sumensari, and R. Zukanovich Funchal, Lepton flavor violation in exclusive $b \rightarrow s$ decays, Eur. Phys. J. C76 (2016), no. 3 134, [arXiv: 1602.00881].

[296] BaBar, J. P. Lees et al., A search for the decay modes $B^{+-} \rightarrow h^{+-} \tau^{+-} l$, Phys. Rev. D86 (2012) 012004, [arXiv:1204.2852].

[297] LHCb, R. Aaij et al., Search for the decays $B_{s}^{0} \rightarrow \tau^{+} \tau^{-}$and $B^{0} \rightarrow \tau^{+} \tau^{-}$, Phys. Rev. Lett. 118 (2017), no. 25 251802, [arXiv:1703.02508].

[298] A. Crivellin, L. Hofer, J. Matias, U. Nierste, S. Pokorski, and J. Rosiek, Lepton-flavour violating $B$ decays in generic $Z^{\prime}$ models, Phys. Rev. D92 (2015), no. 5 054013, [arXiv: 1504.07928].

[299] B. Capdevila, A. Crivellin, S. Descotes-Genon, L. Hofer, and J. Matias, Searching for New Physics with $b \rightarrow s \tau^{+} \tau^{-}$processes, arXiv:1712.01919.

[300] G. Moreau and M. Chemtob, R-parity violation and the cosmological gravitino problem, Phys. Rev. D65 (2002) 024033, [hep-ph/0107286].

[301] F. U. Bernlochner, Z. Ligeti, M. Papucci, and D. J. Robinson, Combined analysis of semileptonic $B$ decays to $D$ and $D^{*}: R\left(D^{(*)}\right),\left|V_{c b}\right|$, and new physics, Phys. Rev. D95 (2017), no. 11 115008, [arXiv:1703.05330]. [Erratum: Phys. Rev.D97,no.5,059902(2018)].

[302] R. Barbieri and A. Strumia, The 'LEP paradox', in 4th Rencontres du Vietnam: Physics at Extreme Energies (Particle Physics and Astrophysics) Hanoi, Vietnam, July 19-25, 2000, 2000. hep-ph/0007265.

[303] Z. Chacko, H.-S. Goh, and R. Harnik, The Twin Higgs: Natural electroweak breaking from mirror symmetry, Phys. Rev. Lett. 96 (2006) 231802, [hep-ph/0506256].

[304] G. Burdman, Z. Chacko, H.-S. Goh, and R. Harnik, Folded supersymmetry and the LEP paradox, JHEP 02 (2007) 009, [hep-ph/0609152].

[305] R. Barbieri, T. Gregoire, and L. J. Hall, Mirror world at the large hadron collider, hep-ph/0509242.

[306] H. Cai, H.-C. Cheng, and J. Terning, A Quirky Little Higgs Model, JHEP 05 (2009) 045, [arXiv:0812.0843]. 
[307] G. Burdman, Z. Chacko, H.-S. Goh, R. Harnik, and C. A. Krenke, The Quirky Collider Signals of Folded Supersymmetry, Phys. Rev. D78 (2008) 075028, [arXiv:0805.4667].

[308] D. Poland and J. Thaler, The Dark Top, JHEP 11 (2008) 083, [arXiv:0808.1290].

[309] P. Batra and Z. Chacko, A Composite Twin Higgs Model, Phys. Rev. D79 (2009) 095012, [arXiv:0811.0394].

[310] N. Craig, S. Knapen, and P. Longhi, Neutral Naturalness from Orbifold Higgs Models, Phys. Rev. Lett. 114 (2015), no. 6 061803, [arXiv:1410.6808].

[311] N. Craig, S. Knapen, and P. Longhi, The Orbifold Higgs, JHEP 03 (2015) 106, [arXiv:1411.7393].

[312] G. Burdman, Z. Chacko, R. Harnik, L. de Lima, and C. B. Verhaaren, Colorless Top Partners, a 125 GeV Higgs, and the Limits on Naturalness, Phys. Rev. D91 (2015), no. 5 055007, [arXiv:1411.3310].

[313] R. Barbieri, D. Greco, R. Rattazzi, and A. Wulzer, The Composite Twin Higgs scenario, JHEP 08 (2015) 161, [arXiv: 1501.07803].

[314] M. Low, A. Tesi, and L.-T. Wang, Twin Higgs mechanism and a composite Higgs boson, Phys. Rev. D91 (2015) 095012, [arXiv:1501.07890].

[315] I. García García, R. Lasenby, and J. March-Russell, Twin higgs wimp dark matter, Phys. Rev. D 92 (Sep, 2015) 055034.

[316] N. Craig and A. Katz, The Fraternal WIMP Miracle, arXiv:1505.07113.

[317] I. García García, R. Lasenby, and J. March-Russell, Twin higgs asymmetric dark matter, Phys. Rev. Lett. 115 (Sep, 2015) 121801.

[318] M. Farina, Asymmetric Twin Dark Matter, arXiv:1506.03520.

[319] B. Batell and M. McCullough, Neutrino Masses from Neutral Top Partners, arXiv: 1504.04016

[320] N. Craig, A. Katz, M. Strassler, and R. Sundrum, Naturalness in the Dark at the LHC, JHEP 07 (2015) 105, [arXiv:1501.05310].

[321] D. Curtin and C. B. Verhaaren, Discovering Uncolored Naturalness in Exotic Higgs Decays, arXiv:1506.06141.

[322] D. Curtin and P. Saraswat, Towards a No-Lose Theorem for Naturalness, arXiv: 1509.04284. 
[323] Z. Chacko, Y. Nomura, M. Papucci, and G. Perez, Natural little hierarchy from a partially goldstone twin Higgs, JHEP 01 (2006) 126, [hep-ph/0510273].

[324] S. Chang, L. J. Hall, and N. Weiner, A Supersymmetric twin Higgs, Phys. Rev. D75 (2007) 035009, [hep-ph/0604076].

[325] A. Falkowski, S. Pokorski, and M. Schmaltz, Twin SUSY, Phys. Rev. D74 (2006) 035003, [hep-ph/0604066].

[326] J. Galloway, M. A. Luty, Y. Tsai, and Y. Zhao, Induced Electroweak Symmetry Breaking and Supersymmetric Naturalness, Phys. Rev. D89 (2014), no. 7 075003, [arXiv:1306.6354].

[327] N. Craig and K. Howe, Doubling down on naturalness with a supersymmetric twin Higgs, JHEP 03 (2014) 140, [arXiv:1312.1341].

[328] Particle Data Group, K. A. Olive et al., Review of Particle Physics, Chin. Phys. C38 (2014) 090001.

[329] ATLAS, CMS, G. Aad et al., Combined Measurement of the Higgs Boson Mass in pp Collisions at $\sqrt{s}=7$ and 8 TeV with the ATLAS and CMS Experiments, Phys. Rev. Lett. 114 (2015) 191803, [arXiv:1503.07589]. 